\title{
Multireference Description of Nickel-Aryl Homolytic Bond Dissociation Processes in Photoredox Catalysis
}

\author{
David Cagan, Gautam Stroscio, Alexander Cusumano, Ryan Hadt
}

Submitted date: 22/09/2020 - Posted date: 23/09/2020

Licence: CC BY-NC-ND 4.0

Citation information: Cagan, David; Stroscio, Gautam; Cusumano, Alexander; Hadt, Ryan (2020): Multireference Description of Nickel-Aryl Homolytic Bond Dissociation Processes in Photoredox Catalysis. ChemRxiv. Preprint. https://doi.org/10.26434/chemrxiv.12990830.v1

Multireference electronic structure calculations consistent with known experimental data have elucidated a novel mechanism for photo-triggered $\mathrm{Ni}(\mathrm{II})-\mathrm{C}$ homolytic bond dissociation in Ni 2,2'-bipyridine (bpy) photoredox catalysts. Previously, a thermally assisted dissociation from the lowest energy triplet ligand field excited state was proposed and supported by density functional theory (DFT) calculations that reveal a barrier of $\sim 30 \mathrm{kcal} \mathrm{mol}^{-1}$. In contrast, multireference ab initio calculations suggest this process is disfavored, with barrier heights of $\sim 70 \mathrm{kcal} \mathrm{mol}^{-1}$, and highlight important ligand noninnocent contributions to excited state relaxation and bond dissociation processes that are not captured with DFT. In the multireference description, photo-triggered $\mathrm{Ni}(\mathrm{II})-\mathrm{C}$ homolytic bond dissociation occurs via initial population of a singlet $\mathrm{Ni}(\mathrm{II})$-to-bpy metal-to-ligand charge transfer ( ${ }^{1} \mathrm{MLCT}$ ) excited state followed by intersystem crossing and aryl-to-Ni(III) charge transfer, overall a formal two-electron transfer process driven by a single photon. This results in repulsive triplet excited states from which spontaneous homolytic bond dissociation can occur, effectively competing with relaxation to the lowest energy, nondissociative triplet $\mathrm{Ni}(\mathrm{II})$ ligand field excited state. These findings guide important electronic structure considerations for the experimental and computational elucidation of the mechanisms of ground and excited state cross-coupling catalysis mediated by $\mathrm{Ni}$ heteroaromatic complexes.

File list (2)

Ni_photo_ChemRxiv.pdf (1.04 MiB) view on ChemRxiv - download file Ni_photo_SI_ChemRxiv.pdf (11.31 MiB) view on ChemRxiv - download file 


\title{
Multireference Description of Nickel-Aryl Homolytic Bond Dissociation Processes in Photoredox Catalysis
}

\author{
David A. Cagan ${ }^{\dagger}$ Gautam D. Stroscio ${ }^{\dagger}$, Alexander Q. Cusumano, Ryan G. Hadt* \\ Division of Chemistry and Chemical Engineering, Arthur Amos Noyes Laboratory of Chemical \\ Physics, California Institute of Technology, Pasadena, California 91125, United States \\ ${ }^{\dagger}$ Co-first authors
}

Corresponding Author: rghadt@,caltech.edu

\begin{abstract}
Multireference electronic structure calculations consistent with known experimental data have elucidated a novel mechanism for photo-triggered $\mathrm{Ni}(\mathrm{II})-\mathrm{C}$ homolytic bond dissociation in $\mathrm{Ni}$ 2,2'bipyridine (bpy) photoredox catalysts. Previously, a thermally assisted dissociation from the lowest energy triplet ligand field excited state was proposed and supported by density functional theory (DFT) calculations that reveal a barrier of $\sim 30 \mathrm{kcal} \mathrm{mol}^{-1}$. In contrast, multireference ab initio calculations suggest this process is disfavored, with barrier heights of $\sim 70 \mathrm{kcal} \mathrm{mol}^{-1}$, and highlight important ligand noninnocent contributions to excited state relaxation and bond dissociation processes that are not captured with DFT. In the multireference description, phototriggered $\mathrm{Ni}(\mathrm{II})-\mathrm{C}$ homolytic bond dissociation occurs via initial population of a singlet $\mathrm{Ni}$ (II)-tobpy metal-to-ligand charge transfer ( ${ }^{1}$ MLCT) excited state followed by intersystem crossing and aryl-to-Ni(III) charge transfer, overall a formal two-electron transfer process driven by a single photon. This results in repulsive triplet excited states from which spontaneous homolytic bond dissociation can occur, effectively competing with relaxation to the lowest energy, nondissociative triplet $\mathrm{Ni}$ (II) ligand field excited state. These findings guide important electronic structure considerations for the experimental and computational elucidation of the mechanisms of ground and excited state cross-coupling catalysis mediated by Ni heteroaromatic complexes.
\end{abstract}




\section{Introduction}

Merging thermal catalysis with photochemistry (i.e., photoredox catalysis) has provided new, more sustainable routes to bond activations and coupling reactions in organic synthesis. ${ }^{1-10}$ An extension of solar energy conversion, photoredox catalysis utilizes photosensitizers to harvest photon energy and transform it into chemical potential to drive single electron transfer (SET) processes to generate reactive high- and/or low-valent species and important organic radicals. However, photoredox reactions feature complex mechanisms that are challenging to elucidate, and our understanding of how photon energy drives organic transformations is therefore still growing.

Beyond SET, photosensitized energy transfer can form photocatalyst excited states that can be uniquely reactive relative to ground states. ${ }^{11-16}$ The photocatalyst can also potentially act as both the light-absorbing and catalytic unit through direct excitation. ${ }^{17,18}$ In direct excitation and energy transfer mediated catalysis, the ultrafast photophysical processes of transition metal excited state relaxation can also contribute to reactivity. ${ }^{9}$ Notably, $\mathrm{Ni}$ (II) complexes of $2,2^{\prime}$-bipyridine (bpy) exhibit photocatalytic activity for coupling reactions using either energy transfer ${ }^{11,19}$ or direct excitation. ${ }^{20}$ Several mechanistic hypotheses have been discussed and are summarized in Figure 1. In one scenario, energy transfer to a $\mathrm{Ni}(\mathrm{II})$-bpy aryl acetate complex induces reductive elimination from a triplet excited state of $\mathrm{Ni}$ (II) (Figure 1A, bottom), originally proposed to be ligand field in nature. ${ }^{11} \mathrm{Ni}$ (II)-to-bpy metal-to-ligand charge transfer (MLCT) excited states have also been suggested to: 1) mediate bimolecular electron transfer to generate $\mathrm{Ni}(\mathrm{I})$ and $\mathrm{Ni}(\mathrm{III})$ species for catalysis, ${ }^{17}$ or 2 ) directly mediate reductive elimination. ${ }^{21}$ The latter consideration encompasses the one-electron oxidatively induced ground state formal $\mathrm{Ni}$ (III) reactivity discovered by Hillhouse and co-workers. ${ }^{22,23}$

Thermally assisted homolytic $\mathrm{Ni}(\mathrm{II})-\mathrm{C}$ bond dissociation from photochemically formed triplet ligand field excited states in $\mathrm{Ni}(\mathrm{II})$-bpy aryl halide complexes has also been proposed. ${ }^{18}$ This process results in the formation of formal $\mathrm{Ni}(\mathrm{I})$ and aryl radicals (Figure 1A, top). While it is unclear whether these species initiate a subsequent $\mathrm{Ni}(\mathrm{I}) / \mathrm{Ni}(\mathrm{III})$ catalytic cycle, this represents an intriguing means to photochemically generate reduced $\mathrm{Ni}$ species and organic radicals for ground state thermal catalysis..$^{18,20}$ Overall, more detailed experimental and theoretical descriptions of the ground state bonding and excited state relaxation processes in Ni-bpy complexes (and other $\mathrm{Ni}$ heteroaromatic complexes ${ }^{24}$ ) are critical for developing synthetic applications. 

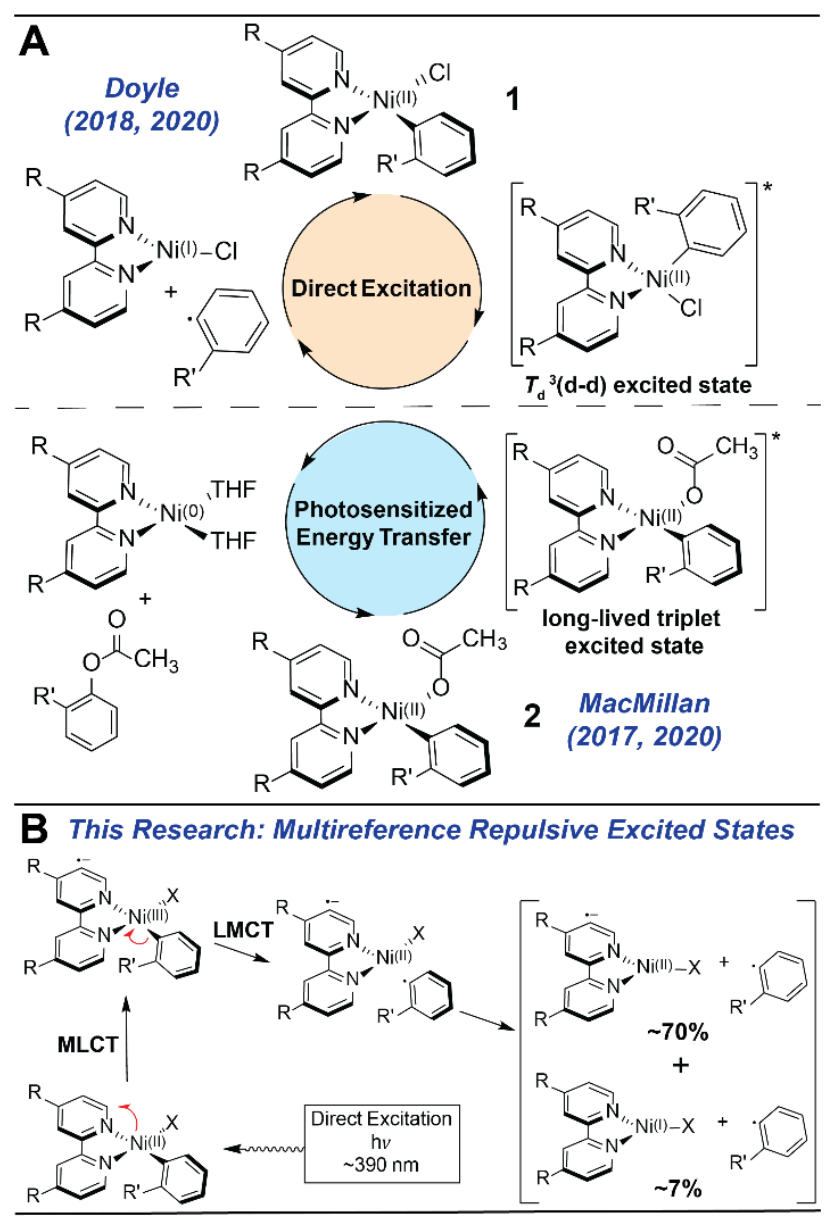

Figure 1. (A) Two previous mechanistic hypotheses related to Ni-bpy photoredox catalysis and (B) findings in this study.

Here we describe a new electronic structural framework to interpret experimental data on $\mathrm{Ni}(\mathrm{II})$-bpy complexes of relevance to photoredox catalysis. Of particular importance is the multireference description (relative to density functional theory (DFT)), which manifests in mixed ground and excited state wave functions and potential energy surfaces (PESs) in $\mathrm{Ni}(\mathrm{II})-\mathrm{C}$ homolytic bond dissociation. Intractable barriers are found for thermal bond dissociation from the lowest energy triplet ligand field excited state within the multireference framework. However, higher energy repulsive triplet excited states are found here and are proposed to be responsible for homolytic bond dissociation. These triplet excited states feature a high-spin Ni(II) coupled to anionic bpy and neutral aryl radicals and can be generated from initial ${ }^{1}$ MLCT excitation (Ni(II)to-bpy) followed by intersystem crossing and intramolecular charge transfer (aryl-to-Ni(III)) (Figure 1B). 


\section{Computational Methods}

Calculations were performed using $\mathrm{ORCA}^{25,26}$ version 4.2.1. The BP86 $6^{27-29}$ functional was used for geometry optimizations and frequency calculations, including both full geometry optimizations and constrained optimizations where the $\mathrm{Ni}-\mathrm{C}$ bond length was systematically varied. The 6$311 \mathrm{G}(\mathrm{d})^{30}$ basis set was used on all atoms, and AutoAux ${ }^{31}$ was used as the auxiliary basis set. SplitRI-J, the default and recommended version of resolution of identities ${ }^{32-35}$ (RI) approximation was used. The finest available DFT grids were used (GRID7 NOFINALGRID). Very tight SCF convergence criteria, which has a convergence tolerance of $10^{-9}$ Hartrees, was applied for all DFT calculations. The restricted Kohn-Sham formalism (RKS) was used for the singlet ground state optimizations; the unrestricted Kohn-Sham formalism (UKS) was used for the triplet optimizations. Additional single point calculations using the B3LYP 28,36 functional, the def2$\mathrm{TZVP}^{37}$ basis, and implicit solvation by tetrahydrofuran (THF) modeled by the conductor-like polarized continuum model ${ }^{38}(\mathrm{CPCM})$ were performed on optimized structures. Here the RIJCOSX ${ }^{39}$ approximation was used with fine DFT grids (GRID7 NOFINALGRID GRIDX9). At this level of theory, broken-symmetry singlet (BSS) and unrestricted triplet single point calculations were performed on the $S=0$ and $S=1$ optimized geometries, respectively. Likewise, TDDFT calculations were performed using these same settings. Applying a Yamaguchi spin correction $^{40,41}$ did not significantly affect the BSS dissociations energies of $\mathbf{1}$ and $\mathbf{2}$. It lowered the dissociation energy of 1 from $43.3 \mathrm{kcal} \mathrm{mol}^{-1}$ to $41.7 \mathrm{kcal} \mathrm{mol}^{-1}$ (Table S1P); it barely changed the dissociation energy of 2 from $44.9 \mathrm{kcal} \mathrm{mol}^{-1}$ to $45.1 \mathrm{kcal} \mathrm{mol}^{-1}$ (Table S2L). Sample input DFT and TDDFT parameters are given on page S3.

Quasidegenerate N-electron valence state second-order perturbation theory ${ }^{42}$ (QDNEVPT2) corrected complete active space self-consistent field (CASSCF) single point calculations were performed on DFT optimized geometries. Tight SCF convergence criteria with an energy tolerance of $10^{-7}$ Hartrees were applied. The def2-TZVP basis set was used on all atoms, and the RIJCOSX approximation was employed. Note that the number of states averaged was varied (see Tables S1G-1 - S1G-3), and it was found that a state-averaging with fifteen singlets and twenty-five triplets yielded a thorough description of the ground and excited states of interest while maintaining reasonable computational costs. Therefore, state-averaged CASSCF/QDNEVPT2 single point calculations utilized fifteen singlets and twenty-five triplets throughout. The recommended Nakano formalism was used, and the corresponding CI-vectors are tabulated below. 
A comparison between gas phase and solvent corrected CASSCF/QD-NEVPT2 single point calculations yielded qualitatively similar results at both the singlet equilibrium geometry and at longer $\mathrm{Ni}-\mathrm{C}$ distances $(3.2 \AA$ for $\mathbf{1}$ and $3.1 \AA$ for $\mathbf{2})$; therefore, gas phase calculations were conducted on all structures (comparisons are tabulated in the Supporting Information). Sample input files for CASSCF/QD-NEVPT2 calculations are given on page S3.

The size of the active space was varied until a thorough description of $\mathbf{1}$ and $\mathbf{2}$ was reached (comparisons between active space sizes are tabulated in the Supporting Information). Active spaces are shown in Figures $\mathrm{S} 1 \mathrm{C}$ and $\mathrm{S} 1 \mathrm{E}$ for $\mathbf{1}(\mathrm{S}=0$ and $\mathrm{S}=1)$ and Figure $\mathrm{S} 2 \mathrm{C}$ and $\mathrm{S} 2 \mathrm{E}$ for $2(\mathrm{~S}=0$ and $\mathrm{S}=1$ ). The first 10-20 lowest energy roots, CI vectors, transitions, and oscillator strengths are tabulated in the Supporting Information. An active space consisting of nine orbitals filled with ten electrons $(90 / 10 e): d(x y), d\left(z^{2}\right), d(x z), d(y z)$, a pair of bonding and antibonding orbitals from the $\mathrm{d}\left(\mathrm{x}^{2}-\mathrm{y}^{2}\right)$ and the $\mathrm{C}\left(\mathrm{sp}^{2}\right)$ orbital on the dissociating phenyl group, and three $\pi^{*}$ orbitals on the bipyridine ligand, were found to be thorough descriptors of the $S=0$ equilibrium geometry of $\mathbf{1}$, while an additional orbital was added for $\mathbf{2}$. The additional orbital in $\mathbf{2}$ is a bonding $\mathrm{d}(\mathrm{xy}) / \mathrm{C}(\boldsymbol{\pi})$ orbital (see Figure S2C), which was kept in the active space due to its partially unfilled occupancy of 1.93 (for compound $\mathbf{1}$, this orbital has occupancy of $\sim 2$ (1.99), and thus was not needed to generate a complete active space). However, as can be seen in Table S2C1-3, the additional orbital in 2 was not involved in any critical transitions. At all other $\mathrm{Ni}-\mathrm{C}$ bond lengths, the third bpy $\pi^{*}$ orbital exhibited very low occupancy and was removed to aid convergence. For example, the ten electrons in nine orbitals CASSCF calculation using the $3.6 \AA$ geometry of $\mathbf{1}$, the third $\pi^{*}$ orbital had an extremely low active space occupancy value of 0.00004 .

As the $\mathrm{Ni}-\mathrm{C}$ bond was elongated and eventually cleaved, the molecular geometry along the singlet surface approached that of the optimized triplet surface. This observation is particularly clear for 2. Geometry coordinates along the $\mathrm{Ni}-\mathrm{C}$ scan are listed in the Appendix portion of the Supporting Information. The active space for the triplet scan of $\mathbf{1}$ again consisted of the ten electrons in eight orbitals (active space with the third $\pi^{*}$ removed, Figure S1E). Here the third bpy $\pi^{*}$ again had very low active space occupancy values $(\sim 0.0001)$. This was true in geometries ranging from 2.0 $\AA$ to $3.6 \AA$. For triplet structures of 1 with a short $\mathrm{Ni}-\mathrm{C}$ bond (between 1.6-2.0 $\AA$ ), the second $\pi^{*}$ orbital was similarly removed to aid convergence. For the triplet scan of $\mathbf{2}$, it was possible to use an active space of ten electrons in nine orbitals active space for the entire scan (Figure S2E). 


\section{Results and Discussion}

Homolytic bond dissociation is an inherently multireference process that can pose difficulties for DFT. ${ }^{43,44}$ Analyses therefore began by comparing the ground state wave functions of $\mathrm{Ni}(\mathrm{II}) \mathrm{t}^{\mathrm{t}}$ ${ }^{\mathrm{Bu}}$ bpy)(o-tolyl) $\mathrm{Cl}$ (1) and $\mathrm{Ni}(\mathrm{II})(\mathrm{bpy})(\mathrm{ph})(\mathrm{ac})(\mathrm{ph}=$ phenyl, ac = acetate) (2), as well as their lowest energy singlet and triplet bond dissociation energies (BDEs) using both DFT and multireference ab initio calculations (i.e., CASSCF/QD-NEVPT2 ${ }^{42}$ ) within ORCA. ${ }^{25,26}$

CASSCF/QD-NEVPT2 calculations on $\mathbf{1}$ and $\mathbf{2}$ exhibit appreciable ground state multireference character (Figures $\mathrm{S} 1 \mathrm{C} / \mathrm{S} 2 \mathrm{C}$ and Tables $\mathrm{S} 1 \mathrm{C} / \mathrm{S} 2 \mathrm{C}-1$ ). Using a nine orbital, ten electron active space (Figure S1C), the dominant contributions to the configuration interaction (CI) vector of the singlet ground state of 1 are $\sim 58 \%$ low-spin $\mathrm{d}^{8}$ (closed shell singlet, CSS) and $\sim 22 \%{ }^{1}$ MLCT (Table S1E). Similar values are obtained for 2 ( $57 \%$ CSS and $\sim 23 \%{ }^{1}$ MLCT) (Table S2D-2). With only an eight electron, five 3d-orbital active space, the low-spin $\mathrm{d}^{8}$ character increases to $\sim 95 \%$ in both 1 and 2 (Table S1A-2/S2B-2). Thus, the unoccupied bpy $\pi^{*}$ orbitals, which have high active space occupancies, play a critical role in the degree of multireference ground state bonding.

It is interesting to consider the multireference data in the context of the DFT bonding description. The low-spin $\mathrm{d}\left(\mathrm{x}^{2}-\mathrm{y}^{2}\right)$ ground states of $\mathbf{1}$ and $\mathbf{2}$ are highly covalent $(\sim 56 / 57 \% \mathrm{Ni}$ (II) and $\sim 11 / 13 \%$ bpy character), with some back-bonding ( $\sim 7-8 \%$ occupied $\mathrm{Ni}$ (II) character in the bpy-based unoccupied $\pi^{*}$ orbitals of both 1 and 2) (Figure S1B/S2B). This highly covalent bonding framework is not particularly amenable to formal redox state assignment and is more consistent with a multireference bonding description. ${ }^{45,46}$

PESs for $\mathrm{Ni}(\mathrm{II})-\mathrm{C}$ bond dissociations from $\mathbf{1}$ and $\mathbf{2}$ are given in Figures $2 \mathrm{~A}$ and S2G, respectively. The DFT BDEs are $\sim 43 \mathrm{kcal} \mathrm{mol}^{-1}$ and $\sim 31 \mathrm{kcal} \mathrm{mol}^{-1}$ starting from the relaxed, lowest energy singlet and triplet structures of $\mathbf{1}$, respectively, consistent with the $\sim 32 \mathrm{kcal} \mathrm{mol}^{-1}$ from a study invoking thermal homolysis on the triplet PES. ${ }^{18}$ Values for 2 are similar $(\sim 45 \mathrm{kcal}$ $\mathrm{mol}^{-1}$ and $\sim 38 \mathrm{kcal} \mathrm{mol}^{-1}$ ). The multireference bond dissociation is fundamentally different than DFT, with significantly higher BDEs $\left(\sim 87 / 65 \mathrm{kcal} \mathrm{mol}^{-1}\right.$ and $73 / 70 \mathrm{kcal} \mathrm{mol}^{-1}$ from the lowest energy singlet and triplet states of $\mathbf{1 / 2}$ ), suggesting the $\mathrm{Ni}(\mathrm{II})-\mathrm{C}$ bonds are stronger than in DFT and will not be thermally cleaved, even upon formation of the relaxed lowest energy triplet ligand 
field excited state. This difference is important, as the $\sim 30 \mathrm{kcal} \mathrm{mol}^{-1}$ barrier was used to rationalize photochemical formation of radicals and reduced Ni species from $1 .^{18}$
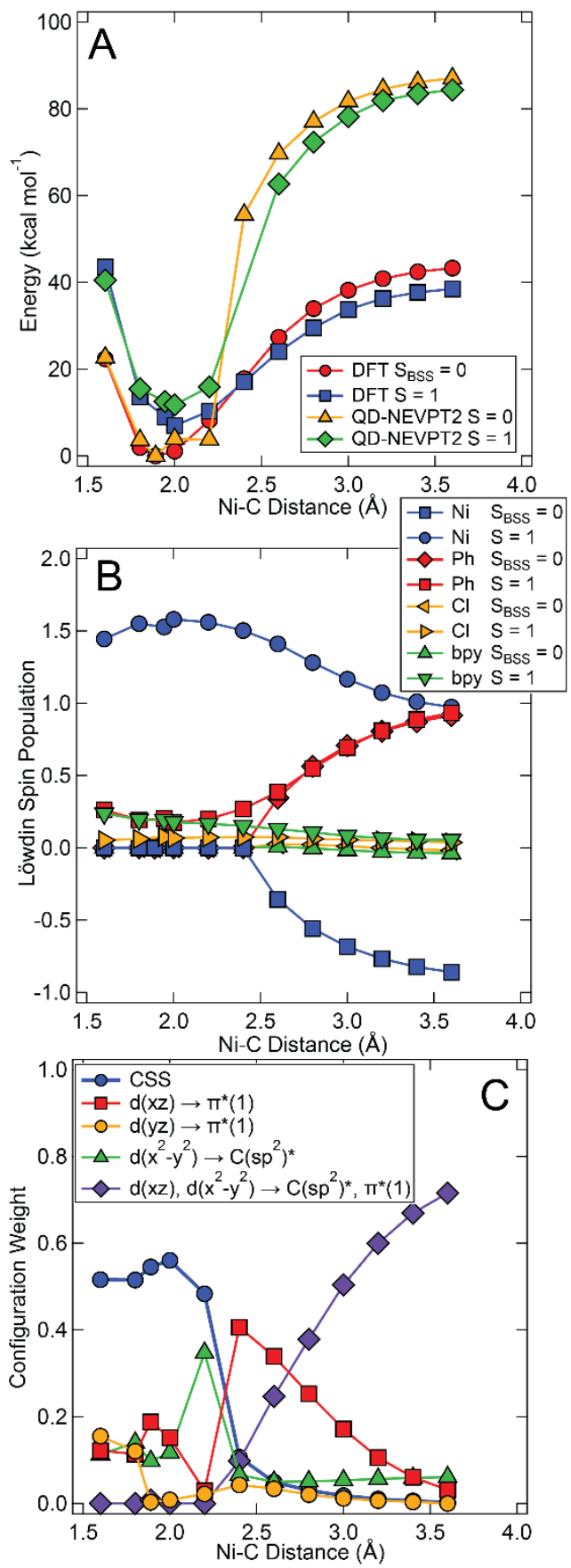

Figure 2. $\mathrm{Ni}(\mathrm{II})-\mathrm{C}$ bond dissociation from the lowest energy singlet and triplet states in 1. (A) Relaxed DFT vs CASSCF/QD-NEVPT2 PESs and (B) DFT Löwdin spin densities for both the singlet (BSS) and triplet states and (C) the CASSCF/QD-NEVPT2 lowest energy singlet CI vector. 
From Löwdin spin density plots in Figures $2 \mathrm{~B}$ and S2H, the DFT-based homolytic bond dissociation results in the formation of $\mathrm{Ni}(\mathrm{I})$ and neutral aryl radicals for $\mathbf{1}$ and $\mathbf{2}$. The compositions of the multireference ground state $\mathrm{CI}$ vectors of $\mathbf{1}$ and $\mathbf{2}$ upon bond dissociation from the singlet ground state are given in Figures 2C and S2I, respectively, and describe the nature of bond homolysis. Upon initial elongation of the $\mathrm{Ni}-\mathrm{C}$ bond, the amount of low-spin $\mathrm{d}^{8}$ character (CSS) decreases significantly, with a concomitant increase in the weighting of ${ }^{1} \mathrm{MLCT}$ character at 2.4 $\AA$, beyond which the $\mathrm{CI}$ vector becomes dominantly $\mathrm{d}(\mathrm{xz}) / \mathrm{d}\left(\mathrm{x}^{2}-\mathrm{y}^{2}\right) \rightarrow \mathrm{C}\left(\mathrm{sp}^{2}\right)^{*} / \pi^{*}$, formally corresponding to a high-spin $\mathrm{Ni}(\mathrm{II})$ coupled to anionic bpy and neutral aryl radicals. Some additional formal $\mathrm{Ni}(\mathrm{I})$ character is also present $(\sim 7 \%)$. Independent DFT vs multireference calculations on the formal $\mathrm{Ni}(\mathrm{I})$ species after homolytic bond dissociation (Figure 3) further support this description. Notably, similar ligand redox has been observed for reduced formal $\mathrm{Ni}(\mathrm{I})$ species in ground state cross-coupling reactions. ${ }^{24,47}$
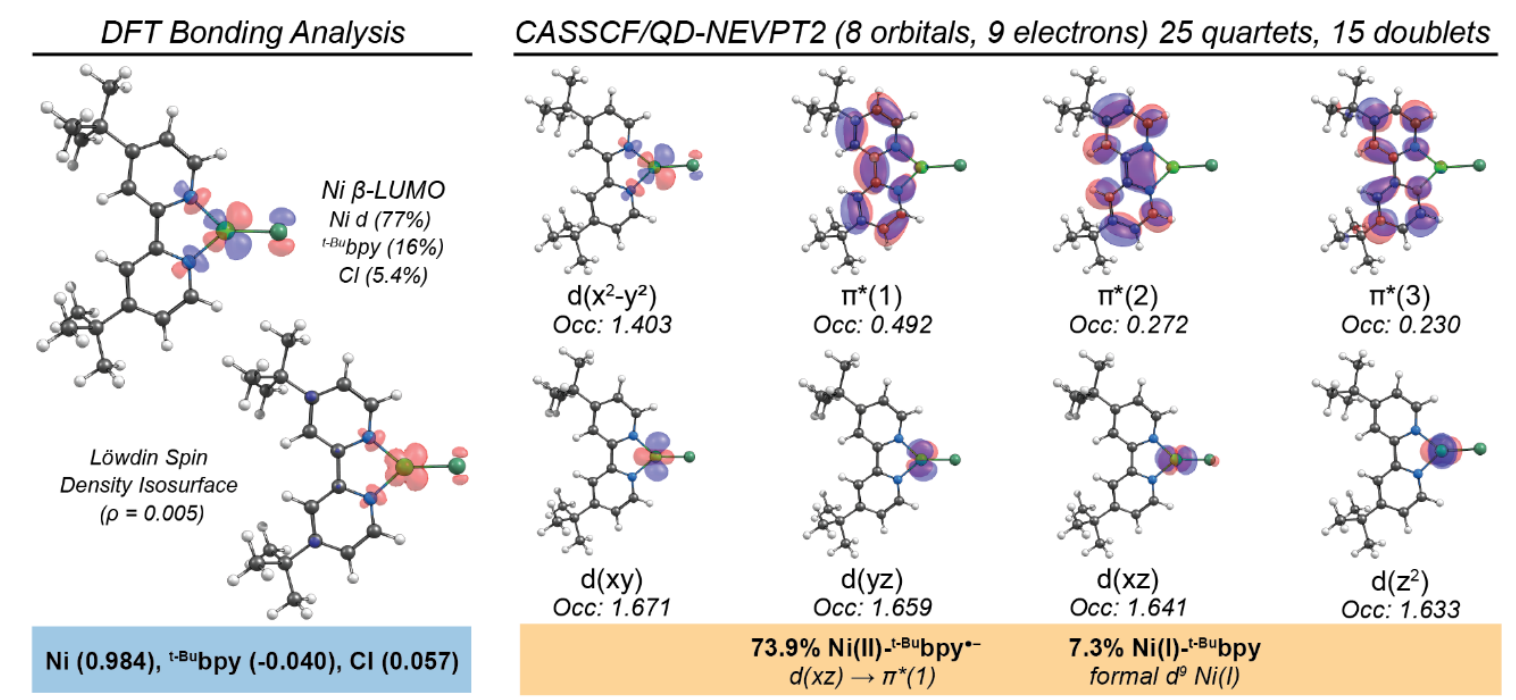

Figure 3. DFT (left) vs CASSCF/QD-NEVPT2 (right) description of the formal Ni(I) species formed upon homolytic $\mathrm{Ni}-\mathrm{C}$ bond cleavage.

Given the intractability of thermally assisted $\mathrm{Ni}(\mathrm{II})-\mathrm{C}$ homolysis and radical formation from the lowest energy triplet ligand field excited states, we now further describe the excited state PESs/manifolds of $\mathbf{1}$ and $\mathbf{2}$ to develop new understanding of the mechanism of homolytic bond dissociation. TDDFT and CASSCF/QD-NEVPT2 calculated excited state manifolds at the ground state singlet relaxed structures of $\mathbf{1}$ and $\mathbf{2}$ are given in Figure S1G and Tables S1I-K, S2A, and 
S2C. Both methods predict a set of lower and higher energy ${ }^{1,3}$ MLCTs. However, their relative oscillator strengths differ somewhat from one another and, for $\mathbf{1}$, the experimental spectrum.

The CASSCF/QD-NEVPT2 ground and excited state PESs along the Ni(II)-C coordinate of $\mathbf{1}$ and $\mathbf{2}$ are given in Figure 4 and S2J, respectively, while the analogous TDDFT PESs are given in Figure S1F and S2K, respectively. From Figure 4, repulsive excited states are present (black, red, and yellow lines). The higher energy MLCT excited states (A in Figure 4) cross the repulsive surfaces at $\mathrm{Ni}-\mathrm{C}$ bond distances of $\sim 2.3-2.4 \AA$ (circled in Figure 4) in both $\mathbf{1}$ and $\mathbf{2}$ with an activation energy from the Franck-Condon point of $\sim 25 \mathrm{kcal} \mathrm{mol}^{-1}$. Thus, the multireference approach predicts homolytic bond dissociation occurs via population of a ${ }^{1}$ MLCT excited state (Ni(II)-to-bpy) followed by an intersystem crossing and intramolecular charge transfer (aryl-to$\mathrm{Ni}(\mathrm{III})$ ) (Figure 1B). The intersystem crossing could occur between the ${ }^{1,3}$ MLCT states or the ${ }^{1}$ MLCT and dissociative triplet state. Overall, this represents a novel homolytic bond dissociation mechanism, which we propose derives from the redox noninnocent and multireference ground and excited state bonding in Ni(II)-bpy complexes.

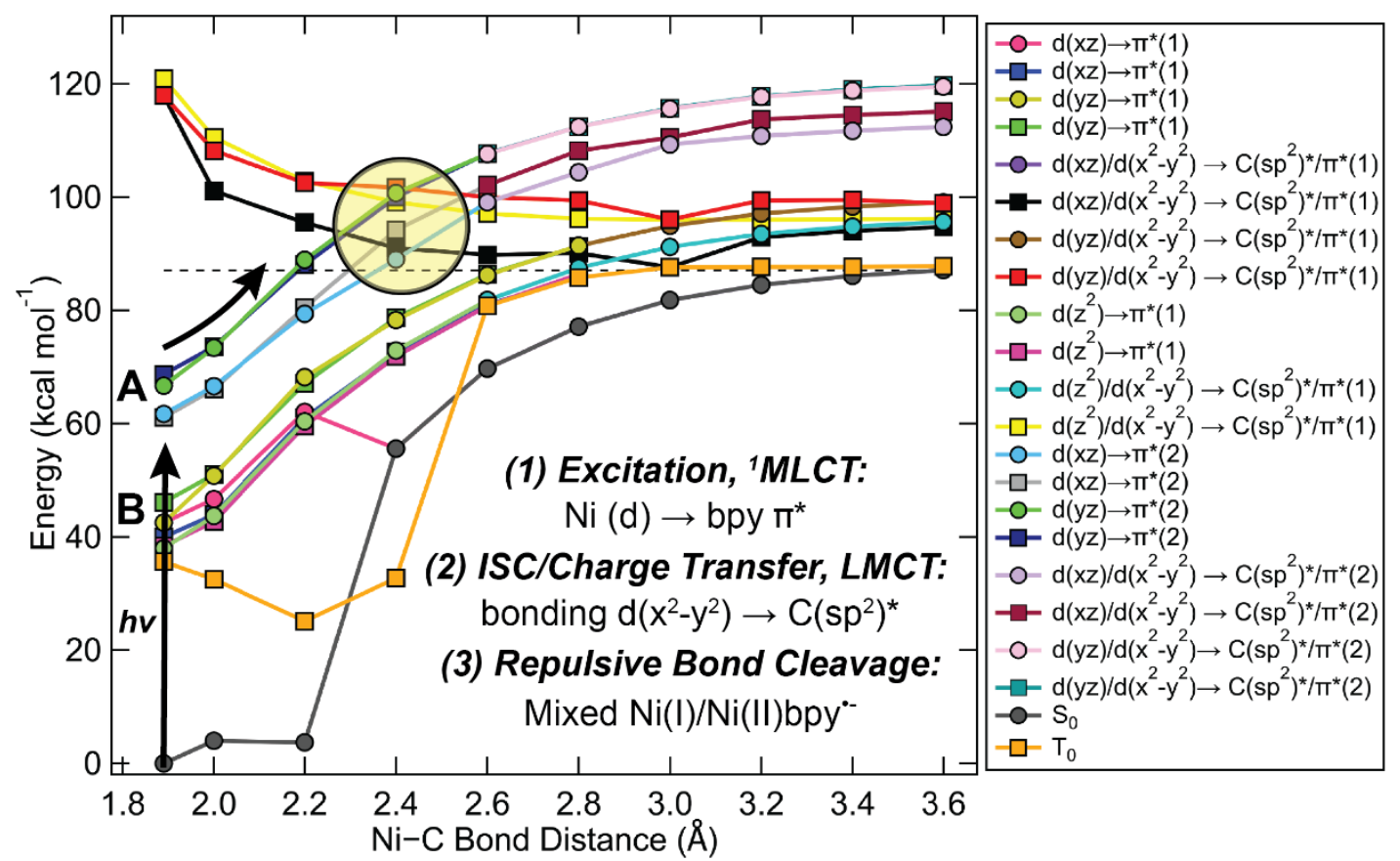

Figure 4. CASSCF/QD-NEVPT2 relaxed ground and excited state PESs along the Ni-C coordinate of $\mathbf{1}$. Vertical excitation (black vertical arrow), the higher (A) and lower energy (B) manifolds of MLCTs, and the crossings between the higher energy MLCTs and repulsive triplets (circled) are depicted. Singlet states, circles; triplets, squares. 
Experimentally, the lowest energy triplet ligand field excited states of Ni(II)-bpy aryl halide complexes are populated in $\sim 5-10$ ps. ${ }^{18}$ Given an estimated Ni(II)-C frequency of $\sim 250$ $\mathrm{cm}^{-1}, \sim 40-80$ vibrational periods could occur to drive intersystem and surface crossings that could compete with population of the lowest energy triplet ligand field state. A higher energy aryl vibration ( $\sim 650 \mathrm{~cm}^{-1}$; Figures S1I/S2L for $\mathbf{1}$ and $\mathbf{2}$, respectively) exhibiting significant changes in $\mathrm{Ni}-\mathrm{C}$ bond distance may also provide $\sim 100-200$ vibrational periods to drive these processes.

The yield of cross-coupled product obtained from direct excitation of $\mathbf{1}$ is incident light dependent; high yields are only observed with UV light $\left(390-395 \mathrm{~nm}\right.$ or $\left.\sim 70 \mathrm{kcal} \mathrm{mol}^{-1}\right),{ }^{20}$ corresponding to excitation into the higher energy manifold of MLCT states (Figure 4). Of particular relevance to compound 2, variations in the energy of the photosensitizer triplet state demonstrated $\mathrm{C}-\mathrm{O}$ coupling occurs when $\sim 40-45 \mathrm{kcal} \mathrm{mol}^{-1}$ is transferred to the Ni catalyst. ${ }^{11}$ This energy would excite complexes to the lower energy manifold of MLCT states (Figure 4, S2J), resulting in thermodynamically unfavorable radical formation $\left(\sim 45 \mathrm{kcal} \mathrm{mol}^{-1}\right)$ for both $\mathbf{1}$ and 2 . Thus, an alternative relaxation pathway and mechanism may exist for photosensitized crosscoupling. In fact, triplet ligand field excited state formation, reductive elimination, and homolytic bond dissociation may all be possible for a given Ni(II)-bpy complex. We believe the ligands in addition to bpy will be of particular importance in determining the relative propensity for specific relaxation pathways. For example, reductive elimination is disfavored for the aryl halide (1) relative to the aryl carboxylate (2). This may preferentially lead to excited state processes that favor the formation of radicals and ligand field excited states over an intractable photosensitized or direct excitation induced reductive elimination. A future study will aim to elucidate and analyze these potentially competitive pathways.

\section{Conclusions}

In summary, we have provided a new electronic structural framework to interpret UV lightinduced homolytic bond dissociation in Ni(II)-bpy complexes of relevance for photoredox catalysis. Compared to DFT, multireference ab initio calculations predict: 1) thermal homolysis from the lowest energy triplet ligand field excited state is not energetically favorable (barriers: DFT $\sim 30 \mathrm{kcal} \mathrm{mol}^{-1}$, CASSCF/QD-NEVPT2 $\sim 70 \mathrm{kcal} \mathrm{mol}^{-1}$ ), 2) initial population of a Ni(II)-tobpy ${ }^{1}$ MLCT excited state can be followed by intersystem crossing and aryl-to-Ni(III) intramolecular charge transfer, resulting in the formation of repulsive triplet excited states 
described as a high-spin $\mathrm{Ni}(\mathrm{II})$ coupled to anionic bpy and neutral aryl radicals. Formally, this represents an overall two-electron transfer process driven by a single photon. The formation of repulsive excited states likely also competes with relaxation to the experimentally observed triplet ligand field excited state, which further relaxes to the ground state without radical formation. 3) The immediate products of homolytic bond dissociation are not Ni(I)-bpy and an aryl radical as described by DFT, but rather, from the multireference CI vector, a dominantly high-spin $\mathrm{Ni}(\mathrm{II})$ coupled to a redox active bpy anion radical ligand, similar to descriptions for formal $\mathrm{Ni}(\mathrm{I})$ intermediates in ground state thermal cross-coupling catalysis. ${ }^{24,47}$

\section{Supporting Information}

Details of computational methods; tabulation of TDDFT and CASSCF/QD-NEVPT2 energetics; tabulation of CASSCF/QD-NEVPT2 CI-vectors; plotted data for $\mathbf{2}$ analogous to data for $\mathbf{1}$ presented in manuscript; DFT optimized structures.

\section{Acknowledgments}

DAC is a National Science Foundation Graduate Research Fellow (DGE-1745301) and is supported by a National Academies of Science, Engineering, and Medicine Ford Foundation Predoctoral Fellowship. Financial support from Caltech and the Dow Next Generation Educator Fund is gratefully acknowledged. 


\section{References}

(1) Nicewicz, D. A.; MacMillan, D. W. C. Merging Photoredox Catalysis with Organocatalysis: The Direct Asymmetric Alkylation of Aldehydes. Science 2008, 322 (5898), 77-80. https://doi.org/10.1126/science.1161976.

(2) Prier, C. K.; Rankic, D. A.; MacMillan, D. W. C. Visible Light Photoredox Catalysis with Transition Metal Complexes: Applications in Organic Synthesis. Chem. Rev. 2013, 113 (7), 5322-5363. https://doi.org/10.1021/cr300503r.

(3) Shaw, M. H.; Twilton, J.; MacMillan, D. W. C. Photoredox Catalysis in Organic Chemistry. J. Org. Chem. 2016, 81 (16), 6898-6926. https://doi.org/10.1021/acs.joc.6b01449.

(4) Twilton, J.; Le, C. (Chip); Zhang, P.; Shaw, M. H.; Evans, R. W.; MacMillan, D. W. C. The Merger of Transition Metal and Photocatalysis. Nat. Rev. Chem. 2017, 1 (7), 0052. https://doi.org/10.1038/s41570-017-0052.

(5) Cavalcanti, L. N.; Molander, G. A. Photoredox Catalysis in Nickel-Catalyzed CrossCoupling. Top. Curr. Chem. 2016, 374 (4), 39. https://doi.org/10.1007/s41061-016-0037-z.

(6) Milligan, J. A.; Phelan, J. P.; Badir, S. O.; Molander, G. A. Alkyl Carbon-Carbon Bond Formation by Nickel/Photoredox Cross-Coupling. Angew. Chem. Int. Ed. 2019, 58 (19), 6152-6163. https://doi.org/10.1002/anie.201809431.

(7) Larsen, C. B.; Wenger, O. S. Photoredox Catalysis with Metal Complexes Made from EarthAbundant Elements. Chem. - Eur. J. 2018, 24 (9), 2039-2058. https://doi.org/10.1002/chem.201703602.

(8) Schultz, D. M.; Yoon, T. P. Solar Synthesis: Prospects in Visible Light Photocatalysis. Science 2014, 343 (6174), 1239176. https://doi.org/10.1126/science.1239176.

(9) Arias-Rotondo, D. M.; McCusker, J. K. The Photophysics of Photoredox Catalysis: A Roadmap for Catalyst Design. Chem. Soc. Rev. 2016, 45 (21), 5803-5820. https://doi.org/10.1039/C6CS00526H.

(10) Yoon, T. P.; Ischay, M. A.; Du, J. Visible Light Photocatalysis as a Greener Approach to Photochemical Synthesis. Nat. Chem. 2010, 2 (7), 527-532. https://doi.org/10.1038/nchem.687.

(11) Welin, E. R.; Le, C.; Arias-Rotondo, D. M.; McCusker, J. K.; MacMillan, D. W. C. Photosensitized, Energy Transfer-Mediated Organometallic Catalysis through Electronically Excited Nickel(II). Science 2017, 355 (6323), 380-385. https://doi.org/10.1126/science.aal2490.

(12) Ischay, M. A.; Anzovino, M. E.; Du, J.; Yoon, T. P. Efficient Visible Light Photocatalysis of [2+2] Enone Cycloadditions. J. Am. Chem. Soc. 2008, 130 (39), 12886-12887. https://doi.org/10.1021/ja805387f.

(13) Lu, Z.; Yoon, T. P. Visible Light Photocatalysis of [2+2] Styrene Cycloadditions by Energy Transfer. Angew. Chem. Int. Ed. 2012, 51 (41), 10329-10332. https://doi.org/10.1002/anie.201204835.

(14) Zhang, Z.; Rogers, C. R.; Weiss, E. A. Energy Transfer from CdS QDs to a Photogenerated Pd Complex Enhances the Rate and Selectivity of a Pd-Photocatalyzed Heck Reaction. $J$. Am. Chem. Soc. 2020, 142 (1), 495-501. https://doi.org/10.1021/jacs.9b11278.

(15) Strieth-Kalthoff, F.; Glorius, F. Triplet Energy Transfer Photocatalysis: Unlocking the Next Level. Chem 2020, 6 (8), 1888-1903. https://doi.org/10.1016/j.chempr.2020.07.010.

(16) Strieth-Kalthoff, F.; J. James, M.; Teders, M.; Pitzer, L.; Glorius, F. Energy Transfer Catalysis Mediated by Visible Light: Principles, Applications, Directions. Chem. Soc. Rev. 2018, 47 (19), 7190-7202. https://doi.org/10.1039/C8CS00054A. 
(17) Shields, B. J.; Kudisch, B.; Scholes, G. D.; Doyle, A. G. Long-Lived Charge-Transfer States of Nickel(II) Aryl Halide Complexes Facilitate Bimolecular Photoinduced Electron Transfer. J. Am. Chem. Soc. 2018, 140 (8), 3035-3039. https://doi.org/10.1021/jacs.7b13281.

(18) Ting, S. I.; Garakyaraghi, S.; Taliaferro, C. M.; Shields, B. J.; Scholes, G. D.; Castellano, F. N.; Doyle, A. G. 3d-d Excited States of Ni(II) Complexes Relevant to Photoredox Catalysis: Spectroscopic Identification and Mechanistic Implications. J. Am. Chem. Soc. 2020, 142 (12), 5800-5810. https://doi.org/10.1021/jacs.0c00781.

(19) Kim, T.; McCarver, S. J.; Lee, C.; MacMillan, D. W. C. Sulfonamidation of Aryl and Heteroaryl Halides through Photosensitized Nickel Catalysis. Angew. Chem. Int. Ed. 2018, 57 (13), 3488-3492. https://doi.org/10.1002/anie.201800699.

(20) Yang, L.; Lu, H.-H.; Lai, C.-H.; Li, G.; Zhang, W.; Cao, R.; Liu, F.; Wang, C.; Xiao, J.; Xue, D. Light-Promoted Nickel Catalysis: Etherification of Aryl Electrophiles with Alcohols Catalyzed by a NiII-Aryl Complex. Angew. Chem. Int. Ed. 2020, 59 (31), 1271412719. https://doi.org/10.1002/anie.202003359.

(21) Ma, P.; Wang, S.; Chen, H. Reactivity of Transition-Metal Complexes in Excited States: CO Bond Coupling Reductive Elimination of a Ni(II) Complex Is Elicited by the Metal-toLigand Charge Transfer State. ACS Catal. 2020, 10 (1), 1-6. https://doi.org/10.1021/acscatal.9b03827.

(22) Koo, K.; Hillhouse, G. L. Carbon-Nitrogen Bond Formation by Reductive Elimination from Nickel(II) Amido Alkyl Complexes. Organometallics 1995, 14 (9), 4421-4423. https://doi.org/10.1021/om00009a054.

(23) Han, R.; Hillhouse, G. L. Carbon-Oxygen Reductive-Elimination from Nickel(II) Oxametallacycles and Factors That Control Formation of Ether, Aldehyde, Alcohol, or Ester Products. J. Am. Chem. Soc. 1997, 119 (34), 8135-8136. https://doi.org/10.1021/ja9714999.

(24) Jones, G. D.; Martin, J. L.; McFarland, C.; Allen, O. R.; Hall, R. E.; Haley, A. D.; Brandon, R. J.; Konovalova, T.; Desrochers, P. J.; Pulay, P.; Vicic, D. A. Ligand Redox Effects in the Synthesis, Electronic Structure, and Reactivity of an Alkyl-Alkyl Cross-Coupling Catalyst. J. Am. Chem. Soc. 2006, 128 (40), 13175-13183. https://doi.org/10.1021/ja063334i.

(25) Neese, F. The ORCA Program System. Wiley Interdiscip. Rev. Comput. Mol. Sci. 2012, 2 (1), 73-78. https://doi.org/10.1002/wcms.81.

(26) Neese, F. Software Update: The ORCA Program System, Version 4.0. Wiley Interdiscip. Rev. Comput. Mol. Sci. 2018, 8 (1), e1327. https://doi.org/10.1002/wcms.1327.

(27) Perdew, J. P. Density-Functional Approximation for the Correlation Energy of the Inhomogeneous Electron Gas. Phys. Rev. B 1986, 33 (12), 8822-8824. https://doi.org/10.1103/PhysRevB.33.8822.

(28) Becke, A. D. Density-Functional Exchange-Energy Approximation with Correct Asymptotic Behavior. Phys. Rev. A 1988, 38 (6), 3098-3100. https://doi.org/10.1103/PhysRevA.38.3098.

(29) Becke, A. D. Density-functional Thermochemistry. III. The Role of Exact Exchange. $J$. Chem. Phys. 1993, 98 (7), 5648-5652. https://doi.org/10.1063/1.464913.

(30) Curtiss, L. A.; McGrath, M. P.; Blaudeau, J.; Davis, N. E.; Binning, R. C.; Radom, L. Extension of Gaussian-2 Theory to Molecules Containing Third-row Atoms Ga-Kr. $J$. Chem. Phys. 1995, 103 (14), 6104-6113. https://doi.org/10.1063/1.470438. 
(31) Stoychev, G. L.; Auer, A. A.; Neese, F. Automatic Generation of Auxiliary Basis Sets. J. Chem. Theory Comput. 2017, 13 (2), 554-562. https://doi.org/10.1021/acs.jctc.6b01041.

(32) Neese, F. An Improvement of the Resolution of the Identity Approximation for the Formation of the Coulomb Matrix. J. Comput. Chem. 2003, 24 (14), 1740-1747. https://doi.org/10.1002/jcc.10318.

(33) Kossmann, S.; Neese, F. Comparison of Two Efficient Approximate Hartee-Fock Approaches. Chem. Phys. Lett. 2009, 481 (4), 240-243. https://doi.org/10.1016/j.cplett.2009.09.073.

(34) Izsák, R.; Neese, F. An Overlap Fitted Chain of Spheres Exchange Method. J. Chem. Phys. 2011, 135 (14), 144105. https://doi.org/10.1063/1.3646921.

(35) Izsák, R.; Neese, F. Speeding up Spin-Component-Scaled Third-Order Pertubation Theory with the Chain of Spheres Approximation: The COSX-SCS-MP3 Method. Mol. Phys. 2013, 111 (9-11), 1190-1195. https://doi.org/10.1080/00268976.2013.796071.

(36) Lee, C.; Yang, W.; Parr, R. G. Development of the Colle-Salvetti Correlation-Energy Formula into a Functional of the Electron Density. Phys. Rev. B 1988, 37 (2), 785-789. https://doi.org/10.1103/PhysRevB.37.785.

(37) Weigend, F.; Ahlrichs, R. Balanced Basis Sets of Split Valence, Triple Zeta Valence and Quadruple Zeta Valence Quality for H to Rn: Design and Assessment of Accuracy. Phys Chem Chem Phys 2005, 7 (18), 3297-3305. https://doi.org/10.1039/B508541A.

(38) Barone, V.; Cossi, M. Quantum Calculation of Molecular Energies and Energy Gradients in Solution by a Conductor Solvent Model. J. Phys. Chem. A 1998, 102 (11), 1995-2001. https://doi.org/10.1021/jp9716997.

(39) Kossmann, S.; Neese, F. Efficient Structure Optimization with Second-Order Many-Body Perturbation Theory: The RIJCOSX-MP2 Method. J. Chem. Theory Comput. 2010, 6 (8), 2325-2338. https://doi.org/10.1021/ct100199k.

(40) Yamaguchi, K.; Takahara, Y.; Fueno, T.; Houk, K. N. Extended Hartree-Fock (EHF) Theory of Chemical Reactions. Theor. Chim. Acta 1988, 73 (5), 337-364. https://doi.org/10.1007/BF00527740.

(41) Kitagawa, Y.; Saito, T.; Nakanishi, Y.; Kataoka, Y.; Matsui, T.; Kawakami, T.; Okumura, M.; Yamaguchi, K. Spin Contamination Error in Optimized Geometry of Singlet Carbene $\left({ }^{1} \mathrm{~A}_{1}\right)$ by Broken-Symmetry Method. J. Phys. Chem. A 2009, 113 (52), 15041-15046. https://doi.org/10.1021/jp905125g.

(42) Angeli, C.; Borini, S.; Cestari, M.; Cimiraglia, R. A Quasidegenerate Formulation of the Second Order N-Electron Valence State Perturbation Theory Approach. J. Chem. Phys. 2004, 121 (9), 4043-4049. https://doi.org/10.1063/1.1778711.

(43) Bao, J. L.; Odoh, S. O.; Gagliardi, L.; Truhlar, D. G. Predicting Bond Dissociation Energies of Transition-Metal Compounds by Multiconfiguration Pair-Density Functional Theory and Second-Order Perturbation Theory Based on Correlated Participating Orbitals and Separated Pairs. J. Chem. Theory Comput. 2017, 13 (2), 616-626. https://doi.org/10.1021/acs.jctc.6b01102.

(44) Cramer, C. J.; Truhlar, D. G. Density Functional Theory for Transition Metals and Transition Metal Chemistry. Phys. Chem. Chem. Phys. 2009, 11 (46), 10757-10816. https://doi.org/10.1039/B907148B.

(45) Wilson, S. A.; Kroll, T.; Decreau, R. A.; Hocking, R. K.; Lundberg, M.; Hedman, B.; Hodgson, K. O.; Solomon, E. I. Iron L-Edge X-Ray Absorption Spectroscopy of Oxy- 
Picket Fence Porphyrin: Experimental Insight into Fe-O2 Bonding. J. Am. Chem. Soc. 2013, 135 (3), 1124-1136. https://doi.org/10.1021/ja3103583.

(46) Yan, J. J.; Kroll, T.; Baker, M. L.; Wilson, S. A.; Decréau, R.; Lundberg, M.; Sokaras, D.; Glatzel, P.; Hedman, B.; Hodgson, K. O.; Solomon, E. I. Resonant Inelastic X-Ray Scattering Determination of the Electronic Structure of Oxyhemoglobin and Its Model Complex. Proc. Natl. Acad. Sci. 2019, 116 (8), 2854-2859. https://doi.org/10.1073/pnas.1815981116.

(47) Diccianni, J. B.; Diao, T. Mechanisms of Nickel-Catalyzed Cross-Coupling Reactions. Trends Chem. 2019, 1 (9), 830-844. https://doi.org/10.1016/j.trechm.2019.08.004. 


\title{
Supporting Information
}

\section{Multireference Description of Nickel-Aryl Homolytic Bond Dissociation Processes in Photoredox Catalysis}

\author{
David A. Cagan ${ }^{\dagger}$, Gautam D. Stroscio ${ }^{\dagger}$, Alexander Q. Cusumano, Ryan G. Hadt* \\ Division of Chemistry and Chemical Engineering, Arthur Amos Noyes Laboratory of Chemical Physics, \\ California Institute of Technology, Pasadena, California, 91125, United States \\ ${ }^{\dagger}$ Co-First Authors \\ *Corresponding Author: rghadt@caltech.edu
}


Table of Contents

A. Example Input Files

B. Tables Part 1: Compound 1................................................................................................ S4

C. Figures Part 1: Compound 1.......................................................................................... S22

D. Tables Part 2: Compound 2 ...................................................................................... S30

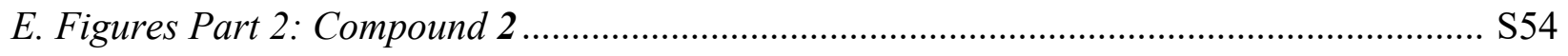

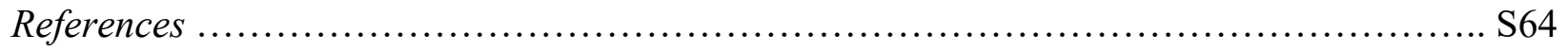

Appendix: DFT Optimized Structures ........................................................................... S66 


\section{A. Example Input Files}

\author{
A.1. Example DFT Input \\ ! RKS BP86 6-311G(d) AutoAux \\ ! TIGHTOPT FREQ \\ ! RI VeryTightSCF GRID7 NOFINALGRID \\ ! SlowConv PrintBasis LargePrint \\ \%pal nprocs 16 \\ end \\ $\%$ maxcore 9000 \\ \%method \\ Z solver DIIS \\ Z shift 0.5 \\ end \\ ${ }^{*}$ xyzfile 01 File.xyz
}

\section{A.2. Example TDDFT Input}

! UKS B3LYP def2-tzvp AutoAux SP RIJCOSX

! VeryTightSCF GRID7 NOFINALGRID GRIDX9

! SlowConv PrintBasis LargePrint CPCM(THF)

! MORead

\%moinp "File.gbw"

\%pal nprocs 16

end

$\%$ maxcore 9000

\%tddft

nroots 50

maxdim 5

end

${ }^{*}$ xyzfile 03 File.xyz

\section{A.3. Example CASSCF/QD-NEVPT2 Input} ! def2-TZVP AutoAux RIJCOSX TightSCF CPCM(THF)

! LargePrint MOREAD

\%moinp "File.gbw"

\%casscf

norb 9

nel 10

mult 3,1

nroots 25,15

TrafoStep RI

Maxlter 400

etol $1 \mathrm{e}-7$

printwf det

orbstep SuperCl

switchstep DIIS

ShiftUp 2.0

ShiftDn 2.0

ptmethod sc_nevpt2

ptsettings

qdtype qd_vanvleck

end

rel

dosoc true

gtensor true

dtensor true

end

end

\%pal

nprocs 16

end

\%maxcore 9000

${ }^{*}$ xyzfile 01 File.xyz 


\section{B. Tables Part 1: Compound 1}

Table S1A-1. Compound 1 - CASSCF/QD-NEVPT2 lowest transition energies in the gas phase (5o, 8e Active Space) - 10 triplet roots, 10 singlet roots. Active Space Orbitals (in order for CI vector notation below): $\mathrm{d}\left(\mathrm{z}^{2}\right), \mathrm{d}(\mathrm{xz}), \mathrm{d}(\mathrm{yz}), \mathrm{d}(\mathrm{xy}), \mathrm{d}\left(\mathrm{x}^{2}-\mathrm{y}^{2}\right)$.

\begin{tabular}{|c|c|c|c|c|c|c|c|}
\hline State & Root & Multiplicity & $\Delta \mathrm{E} / \mathrm{nm}$ & $\Delta \mathrm{E} / \mathrm{kcal} \mathrm{mol}^{-1}$ & CI Vector & $f_{\text {osc }}$ & Contribution \\
\hline \multirow[t]{2}{*}{0} & 0 & 1 & --- & --- & 22220 & -- & 0.95128 \\
\hline & & & & & 02222 & --- & 0.02052 \\
\hline \multirow[t]{2}{*}{1} & 0 & 3 & 1090.8 & 26.2 & 12221 & --- & 0.99927 \\
\hline & & & & & --- & --- & --- \\
\hline \multirow[t]{2}{*}{2} & 1 & 3 & 1009.0 & 28.3 & 21221 & --- & 0.75971 \\
\hline & & & & & 22121 & --- & 0.21841 \\
\hline \multirow[t]{2}{*}{3} & 2 & 3 & 884.4 & 32.3 & 22121 & --- & 0.75325 \\
\hline & & & & & 21221 & --- & 0.21711 \\
\hline \multirow[t]{2}{*}{4} & 3 & 3 & 614.5 & 46.5 & 22211 & --- & 0.93594 \\
\hline & & & & & 21122 & --- & 0.06344 \\
\hline \multirow[t]{2}{*}{5} & 1 & 1 & 420.1 & 68.1 & 21221 & 0.0000024 & 0.77158 \\
\hline & & & & & 22122 & --- & 0.20180 \\
\hline \multirow[t]{2}{*}{6} & 2 & 1 & 414.9 & 68.9 & 12221 & 0.0000399 & 0.97842 \\
\hline & & & & & 22022 & --- & 0.00639 \\
\hline \multirow[t]{2}{*}{7} & 3 & 1 & 395.1 & 72.4 & 22121 & 0.0001334 & 0.77021 \\
\hline & & & & & 21221 & --- & 0.20266 \\
\hline \multirow[t]{2}{*}{8} & 4 & 1 & 382.6 & 74.7 & 22211 & 0.0001352 & 0.99872 \\
\hline & & & & & --- & --- & --- \\
\hline \multirow[t]{2}{*}{9} & 4 & 3 & 292.0 & 97.9 & 21212 & --- & 0.45857 \\
\hline & & & & & 22112 & --- & 0.37735 \\
\hline \multirow[t]{2}{*}{10} & 5 & 3 & 288.9 & 98.9 & 12212 & --- & 0.99393 \\
\hline & & & & & 21122 & --- & 0.00338 \\
\hline
\end{tabular}


Table S1A-2. Compound 1 - CASSCF/QD-NEVPT2 lowest transition energies with CPCM(THF) (5o, 8e Active Space) - 10 triplet roots, 10 singlet roots. Active Space Orbitals (in order for CI vector notation below): $\mathrm{d}\left(\mathrm{z}^{2}\right), \mathrm{d}(\mathrm{xz}), \mathrm{d}(\mathrm{yz}), \mathrm{d}(\mathrm{xy}), \mathrm{d}\left(\mathrm{x}^{2}-\mathrm{y}^{2}\right)$.

\begin{tabular}{|c|c|c|c|c|c|c|c|}
\hline State & Root & Multiplicity & $\Delta \mathrm{E} / \mathrm{nm}$ & $\Delta \mathrm{E} / \mathrm{kcal} \mathrm{mol}^{-1}$ & CI Vector & $f_{\text {osc }}$ & Contribution \\
\hline \multirow[t]{2}{*}{0} & 0 & 1 & --- & --- & 22220 & --- & 0.94984 \\
\hline & & & & & 02222 & --- & 0.02128 \\
\hline \multirow[t]{2}{*}{1} & 0 & 3 & 1224.5 & 23.4 & 12221 & --- & 0.99185 \\
\hline & & & & & --- & --- & --- \\
\hline \multirow[t]{2}{*}{2} & 1 & 3 & 1089.4 & 26.2 & 21221 & --- & 0.94767 \\
\hline & & & & & 22121 & --- & 0.03109 \\
\hline \multirow[t]{2}{*}{3} & 2 & 3 & 973.7 & 29.4 & 22121 & $\begin{array}{c}-- \\
\end{array}$ & 0.93700 \\
\hline & & & & & 21221 & --- & 0.03119 \\
\hline \multirow[t]{2}{*}{4} & 3 & 3 & 633.7 & 45.1 & 22211 & --- & 0.92979 \\
\hline & & & & & 21122 & --- & 0.06922 \\
\hline \multirow[t]{2}{*}{5} & 1 & 1 & 431.4 & 66.3 & 21221 & 0.0000031 & 0.86047 \\
\hline & & & & & 12221 & & 0.09338 \\
\hline \multirow[t]{2}{*}{6} & 2 & 1 & 431.3 & 66.3 & 12221 & 0.0000287 & 0.88620 \\
\hline & & & & & 20222 & & 0.00722 \\
\hline \multirow[t]{2}{*}{7} & 3 & 1 & 410.5 & 69.6 & 22121 & 0.0001197 & 0.94493 \\
\hline & & & & & 21221 & & 0.02702 \\
\hline \multirow[t]{2}{*}{8} & 4 & 1 & 387.8 & 73.7 & 22211 & 0.0001278 & 0.99747 \\
\hline & & & & & --- & & --- \\
\hline \multirow[t]{2}{*}{9} & 4 & 3 & 301.8 & 94.8 & 21212 & --- & 0.59108 \\
\hline & & & & & 22112 & --- & 0.23535 \\
\hline \multirow[t]{2}{*}{10} & 5 & 3 & 299.7 & 95.4 & 12212 & --- & 0.99260 \\
\hline & & & & & 21122 & --- & 0.00415 \\
\hline
\end{tabular}


Table S1B. Compound 1 - CASSCF/QD-NEVPT2 lowest transition energies in the gas phase. 9o, 10e Active Space - 25 triplet roots, 15 singlet roots. Orbitals (in order for CI vector notation below): $d(x y), d\left(x^{2}-y^{2}\right) / C\left(s^{2}\right), d(y z), d\left(z^{2}\right), d(x z), d\left(x^{2}-y^{2}\right) / C\left(s^{2}\right)^{*}, \pi^{*}(1), \pi^{*}(2), \pi^{*}(3)$.

\begin{tabular}{|c|c|c|c|c|c|c|c|}
\hline State & Root & Multiplicity & $\Delta \mathrm{E} / \mathrm{nm}$ & $\Delta \mathrm{E} / \mathrm{kcal} \mathrm{mol}^{-1}$ & CI Vector & $f_{\text {osc }}$ & Contribution \\
\hline \multirow[t]{3}{*}{0} & 0 & 1 & --- & --- & 222220000 & --- & 0.5455 \\
\hline & & & & & 222210100 & --- & 0.1876 \\
\hline & & & & & 212221000 & --- & 0.0977 \\
\hline \multirow[t]{2}{*}{1} & 0 & 3 & 802.3 & 35.6 & 222121000 & --- & 0.4035 \\
\hline & & & & & 222210100 & --- & 0.1917 \\
\hline \multirow[t]{2}{*}{2} & 1 & 3 & 774.8 & 36.9 & 222120100 & --- & 0.6406 \\
\hline & & & & & 222210100 & --- & 0.1186 \\
\hline \multirow[t]{2}{*}{3} & 1 & 1 & 752.5 & 38.0 & 222120100 & 0.0006862 & 0.8166 \\
\hline & & & & & 202122100 & --- & 0.0850 \\
\hline \multirow[t]{2}{*}{4} & 2 & 3 & 733.8 & 39.0 & 221221000 & --- & 0.2244 \\
\hline & & & & & 222210100 & --- & 0.2117 \\
\hline \multirow[t]{2}{*}{5} & 3 & 3 & 715.5 & 40.0 & 221221000 & --- & 0.2533 \\
\hline & & & & & 222210100 & --- & 0.2197 \\
\hline \multirow[t]{2}{*}{6} & 2 & 1 & 672.0 & 42.5 & 221220100 & 0.0649528 & 0.4507 \\
\hline & & & & & 222210100 & --- & 0.2848 \\
\hline \multirow[t]{2}{*}{7} & 4 & 3 & 668.3 & 42.8 & 222211000 & --- & 0.4913 \\
\hline & & & & & 221221000 & --- & 0.2821 \\
\hline \multirow[t]{2}{*}{8} & 5 & 3 & 620.8 & 46.1 & 221220100 & --- & 0.7066 \\
\hline & & & & & 222210100 & --- & 0.0871 \\
\hline \multirow[t]{2}{*}{9} & 3 & 1 & 604.1 & 47.3 & 221220100 & 0.1224866 & 0.3511 \\
\hline & & & & & 222210100 & --- & 0.2756 \\
\hline \multirow[t]{2}{*}{10} & 6 & 3 & 551.6 & 51.8 & 122221000 & --- & 0.6719 \\
\hline & & & & & 221212000 & --- & 0.0803 \\
\hline \multirow[t]{2}{*}{11} & 7 & 3 & 480.7 & 59.5 & 222120010 & --- & 0.7737 \\
\hline & & & & & 202122010 & --- & 0.0829 \\
\hline \multirow[t]{2}{*}{12} & 4 & 1 & 479.5 & 59.6 & 222120010 & 0.0003566 & 0.7222 \\
\hline & & & & & 202122010 & --- & 0.0773 \\
\hline \multirow[t]{2}{*}{13} & 5 & 1 & 472.4 & 60.5 & 122220100 & 0.0001327 & 0.7056 \\
\hline & & & & & 221211100 & --- & 0.0791 \\
\hline \multirow[t]{2}{*}{14} & 8 & 3 & 468.1 & 61.1 & 222210010 & --- & 0.7214 \\
\hline & & & & & 221220010 & --- & 0.0877 \\
\hline \multirow[t]{2}{*}{15} & 6 & 1 & 463.2 & 61.7 & 222210010 & 0.0858212 & 0.3931 \\
\hline & & & & & 221220010 & --- & 0.2037 \\
\hline \multirow[t]{2}{*}{16} & 9 & 3 & 463.0 & 61.8 & 122220100 & --- & 0.7507 \\
\hline & & & & & 221211100 & --- & 0.0792 \\
\hline \multirow[t]{2}{*}{17} & 7 & 1 & 439.3 & 65.1 & 222120001 & 0.0000514 & 0.7108 \\
\hline & & & & & 202122001 & --- & 0.0744 \\
\hline \multirow[t]{2}{*}{18} & 8 & 1 & 428.4 & 66.7 & 221220010 & 0.0799110 & 0.3735 \\
\hline & & & & & 222210001 & --- & 0.3188 \\
\hline \multirow[t]{2}{*}{19} & 10 & 3 & 424.0 & 67.4 & 222120001 & --- & 0.7783 \\
\hline & & & & & 202122001 & --- & 0.0812 \\
\hline \multirow[t]{2}{*}{20} & 11 & 3 & 416.1 & 68.7 & 221220010 & --- & 0.6613 \\
\hline & & & & & 222210010 & --- & 0.0772 \\
\hline
\end{tabular}


Table S1C. Compound 1 - CASSCF/QD-NEVPT2 Lowest transition energies with CPCM(THF) 9o, 10e Active Space - 25 triplet roots, 15 singlet roots. Orbital (in order for CI vector notation below): $\mathrm{d}(\mathrm{xy})$, bonding $\mathrm{d}\left(\mathrm{x}^{2}-\mathrm{y}^{2}\right) / \mathrm{C}\left(\mathrm{sp}^{2}\right), \mathrm{d}(\mathrm{yz}), \mathrm{d}(\mathrm{xz}), \mathrm{d}\left(\mathrm{z}^{2}\right), \mathrm{d}\left(\mathrm{x}^{2}-\mathrm{y}^{2}\right) / \mathrm{C}\left(\mathrm{sp}^{2}\right)^{*}, \pi^{*}(1), \pi^{*}(2), \pi^{*}(3)$.

\begin{tabular}{|c|c|c|c|c|c|c|c|}
\hline State & Root & Multiplicity & $\Delta \mathrm{E} / \mathrm{nm}$ & $\Delta \mathrm{E} / \mathrm{kcal} \mathrm{mol}^{-1}$ & CI Vector & $f_{\text {osc }}$ & Contribution \\
\hline \multirow[t]{3}{*}{0} & 0 & 1 & --- & --- & 222220000 & --- & 0.5752 \\
\hline & & & & & 222120100 & --- & 0.1521 \\
\hline & & & & & 212221000 & --- & 0.1097 \\
\hline \multirow[t]{2}{*}{1} & 0 & 3 & 832.8 & 34.3 & 222211000 & --- & 0.7672 \\
\hline & & & & & 212212000 & --- & 0.1435 \\
\hline \multirow[t]{2}{*}{2} & 1 & 3 & 758.1 & 37.7 & 222121000 & --- & 0.4011 \\
\hline & & & & & 221221000 & --- & 0.3532 \\
\hline \multirow[t]{2}{*}{3} & 2 & 3 & 712.3 & 40.1 & 222210100 & --- & 0.3289 \\
\hline & & & & & 221221000 & --- & 0.3246 \\
\hline \multirow[t]{2}{*}{4} & 3 & 3 & 678.4 & 42.2 & 222210100 & --- & 0.4433 \\
\hline & & & & & 222121000 & --- & 0.2503 \\
\hline \multirow[t]{2}{*}{5} & 1 & 1 & 671.4 & 42.6 & 222210100 & 0.0008408 & 0.8161 \\
\hline & & & & & 202212100 & --- & 0.0876 \\
\hline \multirow[t]{2}{*}{6} & 4 & 3 & 647.1 & 44.2 & 222120100 & --- & 0.7582 \\
\hline & & & & & 202122100 & --- & 0.0850 \\
\hline \multirow[t]{2}{*}{7} & 2 & 1 & 604.2 & 47.3 & 221220100 & 0.0622469 & 0.4193 \\
\hline & & & & & 222120100 & --- & 0.3382 \\
\hline \multirow[t]{2}{*}{8} & 5 & 3 & 574.3 & 49.8 & 221220100 & --- & 0.5752 \\
\hline & & & & & 122221000 & --- & 0.1682 \\
\hline \multirow[t]{2}{*}{9} & 6 & 3 & 551.4 & 51.9 & 122221000 & --- & 0.5491 \\
\hline & & & & & 221220100 & --- & 0.1783 \\
\hline \multirow[t]{2}{*}{10} & 3 & 1 & 546.6 & 52.3 & 221220100 & 0.1520657 & 0.3722 \\
\hline & & & & & 222120100 & --- & 0.2674 \\
\hline \multirow[t]{2}{*}{11} & 4 & 1 & 444.6 & 64.3 & 222120010 & 0.1043472 & 0.5270 \\
\hline & & & & & 221220010 & --- & 0.1267 \\
\hline \multirow[t]{2}{*}{12} & 7 & 3 & 441.9 & 64.7 & 222210010 & --- & 0.7193 \\
\hline & & & & & 222210001 & --- & 0.0917 \\
\hline \multirow[t]{2}{*}{13} & 5 & 1 & 440.4 & 64.9 & 122220100 & 0.0001963 & 0.4135 \\
\hline & & & & & 222210010 & --- & 0.3424 \\
\hline \multirow[t]{2}{*}{14} & 6 & 1 & 437.3 & 65.4 & 222210010 & 0.0003333 & 0.4320 \\
\hline & & & & & 122220100 & --- & 0.3239 \\
\hline \multirow[t]{2}{*}{15} & 8 & 3 & 424.8 & 67.3 & 222120010 & --- & 0.7541 \\
\hline & & & & & 202122010 & --- & 0.0870 \\
\hline \multirow[t]{2}{*}{16} & 9 & 3 & 423.2 & 67.5 & 122220100 & --- & 0.7670 \\
\hline & & & & & 221121100 & --- & 0.0820 \\
\hline \multirow[t]{2}{*}{17} & 10 & 3 & 410.6 & 69.6 & 222210001 & --- & 0.7193 \\
\hline & & & & & 222210010 & --- & 0.0922 \\
\hline \multirow[t]{2}{*}{18} & 7 & 1 & 407.1 & 70.2 & 222121000 & 0.0002537 & 0.6733 \\
\hline & & & & & 221221000 & --- & 0.1149 \\
\hline \multirow[t]{2}{*}{19} & 8 & 1 & 401.3 & 71.3 & 222211000 & 0.0305023 & 0.2808 \\
\hline & & & & & 221220010 & --- & 0.2155 \\
\hline \multirow[t]{2}{*}{20} & 9 & 1 & 399.9 & 71.5 & 122221000 & 0.0329884 & 0.4648 \\
\hline & & & & & 222211000 & --- & 0.1385 \\
\hline
\end{tabular}


Table S1D. Compound 1 - CASSCF/QD-NEVPT2 composition of the singlet equilibrium ground state in gas phase. 9o, 10e Active Space - 25 triplet roots, 15 singlet roots. Orbitals: bonding $\mathrm{d}\left(\mathrm{x}^{2}-\mathrm{y}^{2}\right) / \mathrm{C}\left(\mathrm{sp}^{2}\right), \mathrm{d}(\mathrm{xy}), \mathrm{d}(\mathrm{xz}), \mathrm{d}(\mathrm{yz}), \mathrm{d}\left(\mathrm{z}^{2}\right), \mathrm{d}\left(\mathrm{x}^{2}-\mathrm{y}^{2}\right) / \mathrm{C}\left(\mathrm{sp}^{2}\right)^{*}, \pi^{*}(1), \pi^{*}(2), \pi^{*}(3)$.

\begin{tabular}{|c|c|c|}
\hline CI Vector & Transition & Contribution \\
\hline 222220000 & Closed shell singlet (CSS) $\mathrm{d}^{8}$ & 0.5455 \\
\hline 222210100 & $\mathrm{~d}(\mathrm{xz}) \rightarrow \pi^{*}(1)$ & 0.1876 \\
\hline 212221000 & bonding $\mathrm{d}\left(\mathrm{x}^{2}-\mathrm{y}^{2}\right) / \mathrm{C}\left(\mathrm{sp}^{2}\right) \rightarrow \mathrm{d}\left(\mathrm{x}^{2}-\mathrm{y}^{2}\right) / \mathrm{C}\left(\mathrm{sp}^{2}\right)^{*}$ & 0.0977 \\
\hline 221220001 & $\mathrm{~d}(\mathrm{yz}) \rightarrow \pi^{*}(3)$ & 0.0400 \\
\hline 222210010 & $\mathrm{~d}(\mathrm{xz}) \rightarrow \pi^{*}(2)$ & 0.0312 \\
\hline 202212100 & $2 \mathrm{x}\left[\right.$ bonding $\left.\mathrm{d}\left(\mathrm{x}^{2}-\mathrm{y}^{2}\right) / \mathrm{C}\left(\mathrm{sp}^{2}\right)\right]+\mathrm{d}(\mathrm{xz}) \rightarrow 2 \mathrm{x}\left[\mathrm{d}\left(\mathrm{x}^{2}-\mathrm{y}^{2}\right) / \mathrm{C}\left(\mathrm{sp}^{2}\right)^{*}\right]+\pi^{*}(1)$ & 0.0224 \\
\hline 222121000 & $\left.\mathrm{~d}\left(\mathrm{z}^{2}\right) \rightarrow \mathrm{d}\left(\mathrm{x}^{2}-\mathrm{y}^{2}\right)\right]$ & 0.0090 \\
\hline 212211100 & bonding $\mathrm{d}\left(\mathrm{x}^{2}-\mathrm{y}^{2}\right) / \mathrm{C}\left(\mathrm{sp}^{2}\right)+\mathrm{d}(\mathrm{xz}) \rightarrow \mathrm{d}\left(\mathrm{x}^{2}-\mathrm{y}^{2}\right) / \mathrm{C}\left(\mathrm{sp}^{2}\right)^{*}+\pi^{*}(1)$ & 0.0083 \\
\hline 222022000 & $2 \mathrm{x}\left[\mathrm{d}\left(\mathrm{z}^{2}\right)\right] \rightarrow 2 \mathrm{x}\left[\mathrm{d}\left(\mathrm{x}^{2}-\mathrm{y}^{2}\right) / \mathrm{C}\left(\mathrm{sp}^{2}\right)^{*}\right]$ & 0.0079 \\
\hline 220222000 & $2 \mathrm{x}[\mathrm{d}(\mathrm{yz})] \rightarrow 2 \mathrm{x}\left[\mathrm{d}\left(\mathrm{x}^{2}-\mathrm{y}^{2}\right) / C\left(\mathrm{sp}^{2}\right)^{*}\right]$ & 0.0056 \\
\hline 222202000 & $2 \mathrm{x}[\mathrm{d}(\mathrm{xz})] \rightarrow 2 \mathrm{x}\left[\mathrm{d}\left(\mathrm{x}^{2}-\mathrm{y}^{2}\right) / \mathrm{C}\left(\mathrm{sp}^{2}\right)^{*}\right]$ & 0.0055 \\
\hline 201222001 & $2 \mathrm{x}\left[\right.$ bonding $\left.\mathrm{d}\left(\mathrm{x}^{2}-\mathrm{y}^{2}\right) / \mathrm{C}\left(\mathrm{sp}^{2}\right)\right]+\mathrm{d}(\mathrm{yz}) \rightarrow \mathrm{d}\left(\mathrm{x}^{2}-\mathrm{y}^{2}\right) / \mathrm{C}\left(\mathrm{sp}^{2}\right)^{*}+\pi^{*}(3)$ & 0.0042 \\
\hline 202212010 & $2 \mathrm{x}\left[\right.$ bonding $\left.\mathrm{d}\left(\mathrm{x}^{2}-\mathrm{y}^{2}\right) / \mathrm{C}\left(\mathrm{sp}^{2}\right)\right]+\mathrm{d}(\mathrm{xz}) \rightarrow \mathrm{d}\left(\mathrm{x}^{2}-\mathrm{y}^{2}\right) / \mathrm{C}\left(\mathrm{sp}^{2}\right)^{*}+\pi^{*}(2)$ & 0.0040 \\
\hline 122221000 & $\mathrm{~d}(\mathrm{xz}) \rightarrow \mathrm{d}\left(\mathrm{x}^{2}-\mathrm{y}^{2}\right) / \mathrm{C}\left(\mathrm{sp}^{2}\right)^{*}$ & 0.0029 \\
\hline 221220100 & $\mathrm{~d}(\mathrm{yz}) \rightarrow \pi^{*}(1)$ & 0.0029 \\
\hline \multicolumn{2}{|c|}{ Sum of CSS } & $55 \%$ \\
\hline \multicolumn{2}{|c|}{ Sum of all MLCT } & $26 \%$ \\
\hline \multicolumn{2}{|c|}{ Sum of all d-d } & $13 \%$ \\
\hline \multicolumn{2}{|c|}{ Sum of mixed MLCT + d-d } & $3.9 \%$ \\
\hline
\end{tabular}

Table S1E. Compound 1 - CASSCF/QD-NEVPT2 composition of the singlet equilibrium ground state with CPCM(THF). 9o, 10e Active Space - 25 triplet roots, 15 singlet roots. Orbitals: bonding $\mathrm{d}\left(\mathrm{x}^{2}-\mathrm{y}^{2}\right) / \mathrm{C}\left(\mathrm{sp}^{2}\right), \mathrm{d}(\mathrm{xy}), \mathrm{d}(\mathrm{xz}), \mathrm{d}(\mathrm{yz}), \mathrm{d}\left(\mathrm{z}^{2}\right), \mathrm{d}\left(\mathrm{x}^{2}-\mathrm{y}^{2}\right) / \mathrm{C}\left(\mathrm{sp}^{2}\right)^{*}, \pi^{*}(1), \pi^{*}(2), \pi^{*}(3)$.

\begin{tabular}{|c|c|c|}
\hline CI Vector & Transition & Contribution \\
\hline 222220000 & Closed shell singlet (CSS) $\mathrm{d}^{8}$ & 0.5752 \\
\hline 222120100 & $\mathrm{~d}(\mathrm{xz}) \rightarrow \pi^{*}(1)$ & 0.1521 \\
\hline 212221000 & bonding $\mathrm{d}\left(\mathrm{x}^{2}-\mathrm{y}^{2}\right) / \mathrm{C}\left(\mathrm{sp}^{2}\right) \rightarrow \mathrm{d}\left(\mathrm{x}^{2}-\mathrm{y}^{2}\right) / \mathrm{C}\left(\mathrm{sp}^{2}\right)^{*}$ & 0.1097 \\
\hline 221220001 & $\mathrm{~d}(\mathrm{yz}) \rightarrow \pi^{*}(3)$ & 0.0383 \\
\hline 222120010 & $\mathrm{~d}(\mathrm{xz}) \rightarrow \pi^{*}(2)$ & 0.0256 \\
\hline 202122100 & $2 \mathrm{x}\left[\right.$ bonding $\left.\mathrm{d}\left(\mathrm{x}^{2}-\mathrm{y}^{2}\right) / \mathrm{C}\left(\mathrm{sp}^{2}\right)\right]+\mathrm{d}(\mathrm{xz}) \rightarrow 2 \mathrm{x}\left[\mathrm{d}\left(\mathrm{x}^{2}-\mathrm{y}^{2}\right) / \mathrm{C}\left(\mathrm{sp}^{2}\right)^{*}\right]+\pi^{*}(1)$ & 0.0192 \\
\hline 222211000 & $\mathrm{~d}\left(\mathrm{z}^{2}\right) \rightarrow \mathrm{d}\left(\mathrm{x}^{2}-\mathrm{y}^{2}\right) / \mathrm{C}\left(\mathrm{sp}^{2}\right)$ & 0.0101 \\
\hline 221220100 & $\mathrm{~d}(\mathrm{yz}) \rightarrow \pi^{*}(1)$ & 0.0090 \\
\hline 222202000 & $2 \mathrm{x}\left[\mathrm{d}\left(\mathrm{z}^{2}\right)\right] \rightarrow 2 \mathrm{x}\left[\mathrm{d}\left(\mathrm{x}^{2}-\mathrm{y}^{2}\right) / \mathrm{C}\left(\mathrm{sp}^{2}\right)^{*}\right]$ & 0.0090 \\
\hline 212121100 & bonding $\mathrm{d}\left(\mathrm{x}^{2}-\mathrm{y}^{2}\right) / \mathrm{C}\left(\mathrm{sp}^{2}\right)+\mathrm{d}(\mathrm{xz}) \rightarrow \mathrm{d}\left(\mathrm{x}^{2}-\mathrm{y}^{2}\right) / \mathrm{C}\left(\mathrm{sp}^{2}\right)^{*}+\pi^{*}(1)$ & 0.0073 \\
\hline 220222000 & $2 \mathrm{x}[\mathrm{d}(\mathrm{yz})] \rightarrow 2 \mathrm{x}\left[\mathrm{d}\left(\mathrm{x}^{2}-\mathrm{y}^{2}\right) / \mathrm{C}\left(\mathrm{sp}^{2}\right)^{*}\right]$ & 0.0063 \\
\hline 222022000 & $2 \mathrm{x}[\mathrm{d}(\mathrm{xz})] \rightarrow 2 \mathrm{x}\left[\mathrm{d}\left(\mathrm{x}^{2}-\mathrm{y}^{2}\right) / \mathrm{C}\left(\mathrm{sp}^{2}\right)^{*}\right]$ & 0.0063 \\
\hline 201222001 & $2 \mathrm{x}\left[\right.$ bonding $\left.\mathrm{d}\left(\mathrm{x}^{2}-\mathrm{y}^{2}\right) / \mathrm{C}\left(\mathrm{sp}^{2}\right)\right]+\mathrm{d}(\mathrm{yz}) \rightarrow 2 \mathrm{x}\left[\mathrm{d}\left(\mathrm{x}^{2}-\mathrm{y}^{2}\right) / \mathrm{C}\left(\mathrm{sp}^{2}\right)^{*}\right]+\pi^{*}(3)$ & 0.0041 \\
\hline 202122010 & $2 \mathrm{x}\left[\right.$ bonding $\left.\mathrm{d}\left(\mathrm{x}^{2}-\mathrm{y}^{2}\right) / \mathrm{C}\left(\mathrm{sp}^{2}\right)\right]+\mathrm{d}(\mathrm{xz}) \rightarrow 2 \mathrm{x}\left[\mathrm{d}\left(\mathrm{x}^{2}-\mathrm{y}^{2}\right) / \mathrm{C}\left(\mathrm{sp}^{2}\right)^{*}\right]+\pi^{*}(2)$ & 0.0034 \\
\hline \multicolumn{2}{|l|}{ Sum of CSS } & $\mathbf{5 8 \%}$ \\
\hline \multicolumn{2}{|c|}{ Sum of all MLCT } & $22 \%$ \\
\hline \multirow{2}{*}{\multicolumn{2}{|c|}{$\begin{array}{l}\text { Sum of all d-d } \\
\text { Sum of mixed MLCT }+d-d\end{array}$}} & $14 \%$ \\
\hline & & $3.4 \%$ \\
\hline
\end{tabular}


Table S1F. Compound 1 with Ni-C distance of $3.20 \AA-$ CASSCF/QD-NEVPT2 lowest transition energies in gas phase. $80,10 \mathrm{e}$ Active Space -25 triplet roots, 15 singlet roots. Orbitals: $\mathrm{d}(\mathrm{xy})$, $\mathrm{d}(\mathrm{yz}), \mathrm{d}\left(\mathrm{z}^{2}\right), \mathrm{d}(\mathrm{xz})$, bonding $\mathrm{d}\left(\mathrm{x}^{2}-\mathrm{y}^{2}\right) / \mathrm{C}\left(\mathrm{sp}^{2}\right), \mathrm{d}\left(\mathrm{x}^{2}-\mathrm{y}^{2}\right) / \mathrm{C}\left(\mathrm{sp}^{2}\right)^{*}, \pi^{*}(1), \pi^{*}(2)$.

\begin{tabular}{|c|c|c|c|c|c|c|c|}
\hline State & Root & Multiplicity & $\Delta \mathrm{E} / \mathbf{n m}$ & $\Delta \mathrm{E} / \mathrm{kcal} \mathrm{mol}^{-1}$ & CI Vector & $f_{\text {osc }}$ & Contribution \\
\hline \multirow[t]{3}{*}{0} & 0 & 3 & -- & -- & 22211110 & --- & 0.5998 \\
\hline & & & & & 22212010 & --- & 0.1057 \\
\hline & & & & & 22221100 & --- & 0.0567 \\
\hline \multirow[t]{2}{*}{1} & 0 & 3 & 9086.0 & 3.1 & 22211110 & -- & 0.7446 \\
\hline & & & & & 22112110 & --- & 0.0600 \\
\hline \multirow[t]{2}{*}{2} & 1 & 3 & 3630.2 & 7.9 & 22121110 & --- & 0.8144 \\
\hline & & & & & 22122010 & --- & 0.1216 \\
\hline \multirow[t]{2}{*}{3} & 2 & 3 & 3417.1 & 8.4 & 22211110 & --- & 0.7561 \\
\hline & & & & & 22212010 & --- & 0.0919 \\
\hline \multirow[t]{2}{*}{4} & 1 & 1 & 3189.5 & 9.0 & 22121110 & 0.0000059 & 0.8124 \\
\hline & & & & & 22122010 & --- & 0.1225 \\
\hline \multirow[t]{2}{*}{5} & 3 & 3 & 2489.9 & 11.5 & 22121110 & -- & 0.9789 \\
\hline & & & & & 12221110 & --- & 0.0509 \\
\hline \multirow[t]{2}{*}{6} & 4 & 3 & 2304.7 & 12.4 & 21221110 & --- & 0.7601 \\
\hline & & & & & 21222010 & --- & 0.1103 \\
\hline \multirow[t]{2}{*}{7} & 2 & 1 & 2285.4 & 12.5 & 21221110 & 0.0000005 & 0.6956 \\
\hline & & & & & 21222010 & --- & 0.1058 \\
\hline \multirow[t]{2}{*}{8} & 5 & 3 & 1919.3 & 14.9 & 21221110 & $\begin{array}{ll}-- \\
\end{array}$ & 0.8607 \\
\hline & & & & & 22211110 & --- & 0.0484 \\
\hline \multirow[t]{2}{*}{9} & 6 & 3 & 1172.8 & 24.4 & 22220110 & --- & 0.7851 \\
\hline & & & & & 22221010 & --- & 0.0762 \\
\hline \multirow[t]{2}{*}{10} & 3 & 1 & 1170.0 & 24.4 & 22220110 & 0.0000682 & 0.7229 \\
\hline & & & & & 22221010 & --- & 0.0724 \\
\hline \multirow[t]{2}{*}{11} & 4 & 1 & 1097.4 & 26.1 & 22211101 & 0.0222545 & 0.2581 \\
\hline & & & & & 12221110 & --- & 0.2436 \\
\hline \multirow[t]{2}{*}{12} & 5 & 1 & 1090.0 & 26.2 & 22211101 & 0.0361406 & 0.4130 \\
\hline & & & & & 21212110 & --- & 0.1455 \\
\hline \multirow[t]{2}{*}{13} & 7 & 3 & 1061.0 & 27.0 & 12221110 & --- & 0.4604 \\
\hline & & & & & 21212110 & --- & 0.4285 \\
\hline \multirow[t]{2}{*}{14} & 8 & 3 & 1030.4 & 27.7 & 22211101 & --- & 0.7389 \\
\hline & & & & & 22212001 & --- & 0.0804 \\
\hline \multirow[t]{2}{*}{15} & 9 & 3 & 1003.5 & 28.5 & 12221110 & -- & 0.4832 \\
\hline & & & & & 21212110 & --- & 0.4057 \\
\hline \multirow[t]{2}{*}{16} & 10 & 3 & 994.6 & 28.8 & 22121101 & -- & 0.8005 \\
\hline & & & & & 22122001 & --- & 0.1199 \\
\hline \multirow[t]{2}{*}{17} & 6 & 1 & 993.3 & 28.8 & 22121101 & 0.0000753 & 0.8085 \\
\hline & & & & & 22122001 & --- & 0.1212 \\
\hline \multirow[t]{2}{*}{18} & 11 & 3 & 979.4 & 29.2 & 22211101 & -- & 0.8202 \\
\hline & & & & & 22112101 & --- & 0.0673 \\
\hline \multirow[t]{2}{*}{19} & 12 & 3 & 911.0 & 31.4 & 22121101 & --- & 0.9880 \\
\hline & & & & & 12221101 & --- & 0.0051 \\
\hline \multirow[t]{2}{*}{20} & 7 & 1 & 863.0 & 33.1 & 21221101 & 0.0022399 & 0.7256 \\
\hline & & & & & 21222001 & --- & 0.1076 \\
\hline
\end{tabular}


Table S1G-1. Compound 1 - CASSCF/QD-NEVPT2 composition of the singlet equilibrium ground state while varying number of singlet roots. 9o, 10e Active Space -25 triplet roots, $X$ singlet roots (where $X$ ranged from 15 to 40$)$. Orbitals: bonding $\mathrm{d}\left(\mathrm{x}^{2}-\mathrm{y}^{2}\right) / \mathrm{C}\left(\mathrm{sp}^{2}\right), \mathrm{d}(\mathrm{xy}), \mathrm{d}(\mathrm{xz}), \mathrm{d}(\mathrm{yz})$, $\mathrm{d}\left(\mathrm{z}^{2}\right), \mathrm{d}\left(\mathrm{x}^{2}-\mathrm{y}^{2}\right) / \mathrm{C}\left(\mathrm{sp}^{2}\right)^{*}, \pi^{*}(1), \pi^{*}(2), \pi^{*}(3)$.

\begin{tabular}{|c|c|c|c|c|c|c|c|}
\hline \multicolumn{8}{|c|}{ Weights of Three Most Dominant Terms in CI-Vector (\# Triplets / \# Singlets) } \\
\hline Configuration & 25/15 CPCM & 25/15 Gas & 25/20 Gas & 25/25 Gas & 25/30 Gas & 25/35 Gas & 25/40 Gas \\
\hline CSS & 0.5752 & 0.5455 & 0.5346 & 0.5202 & 0.5029 & 0.4853 & 0.4733 \\
\hline $\begin{array}{l}\mathrm{d}(\mathrm{xz}) \rightarrow \pi^{*}(1) \\
\text { bonding } \mathrm{d}\left(\mathrm{x}^{2}-\mathrm{y}^{2}\right) / \mathrm{C}\left(\mathrm{sp}^{2}\right)\end{array}$ & 0.1521 & 0.1876 & 0.1149 & 0.1321 & 0.1224 & 0.1316 & 0.1198 \\
\hline$\rightarrow \mathrm{d}\left(\mathrm{x}^{2}-\mathrm{y}^{2}\right) / \mathrm{C}\left(\mathrm{sp}^{2}\right)^{*}$ & 0.1097 & 0.0977 & 0.1101 & 0.0853 & 0.0971 & 0.1276 & 0.1430 \\
\hline \multicolumn{8}{|l|}{ Sums of Weights } \\
\hline Configuration & 25/15 CРCM & 25/15 Gas & 25/20 Gas & 25/25 Gas & 25/30 Gas & $25 / 35$ Gas & $25 / 40$ Gas \\
\hline CSS & $58 \%$ & $55 \%$ & $53 \%$ & $52 \%$ & $50 \%$ & $49 \%$ & $47 \%$ \\
\hline Sum of MLCT & $23 \%$ & $26 \%$ & $25 \%$ & $24 \%$ & $23 \%$ & $26 \%$ & $25 \%$ \\
\hline Sum of d-d & $14 \%$ & $13 \%$ & $16 \%$ & $19 \%$ & $21 \%$ & $21 \%$ & $23 \%$ \\
\hline Sum of MLCT $+d-d$ & $3.4 \%$ & $3.9 \%$ & $2.6 \%$ & $1.9 \%$ & $1.7 \%$ & $2.8 \%$ & $2.8 \%$ \\
\hline \multicolumn{8}{|c|}{ Singlet $\rightarrow$ Singlet Transition Energies $\left(\mathrm{kcal} \mathrm{mol}^{-1}\right)$} \\
\hline Transition & 25/15 СРCM & 25/15 Gas & 25/20 Gas & 25/25 Gas & 25/30 Gas & 25/35 Gas & 25/40 Gas \\
\hline $\mathrm{S}_{0} \rightarrow \mathrm{S}_{1}$ & 42.6 & 38.0 & 39.3 & 40.1 & 40.6 & 39.3 & 39.8 \\
\hline $\mathrm{S}_{0} \rightarrow \mathrm{S}_{2}$ & 47.3 & 42.5 & 43.3 & 43.7 & 43.9 & 43.2 & 43.4 \\
\hline $\mathrm{S}_{0} \rightarrow \mathrm{S}_{3}$ & 52.3 & 47.3 & 48.0 & 48.9 & 49.5 & 48.2 & 48.3 \\
\hline $\mathrm{S}_{0} \rightarrow \mathrm{S}_{4}$ & 64.3 & 59.6 & 60.9 & 62.1 & 62.6 & 61.2 & 61.7 \\
\hline $\mathrm{S}_{0} \rightarrow \mathrm{S}_{5}$ & 64.9 & 60.5 & 61.9 & 62.5 & 63.0 & 61.8 & 62.1 \\
\hline $\mathrm{S}_{0} \rightarrow \mathrm{S}_{6}$ & 65.4 & 61.7 & 62.3 & 63.4 & 63.4 & 63.2 & 63.0 \\
\hline $\mathrm{S}_{0} \rightarrow \mathrm{S}_{7}$ & 70.2 & 65.1 & 67.7 & 67.7 & 67.3 & 68.0 & 67.9 \\
\hline $\mathrm{S}_{0} \rightarrow \mathrm{S}_{8}$ & 71.3 & 66.7 & 68.6 & 69.2 & 69.0 & 68.4 & 68.1 \\
\hline $\mathrm{S}_{0} \rightarrow \mathrm{S}_{9}$ & 71.5 & 69.4 & 68.8 & 70.1 & 70.3 & 69.4 & 69.7 \\
\hline $\mathrm{S}_{0} \rightarrow \mathrm{S}_{10}$ & 72.3 & 72.2 & 71.1 & 70.6 & 70.7 & 71.3 & 71.7 \\
\hline $\mathrm{S}_{0} \rightarrow \mathrm{S}_{11}$ & 73.3 & 73.4 & 71.8 & 72.0 & 72.5 & 72.0 & 72.0 \\
\hline $\mathrm{S}_{0} \rightarrow \mathrm{S}_{12}$ & 77.0 & 75.0 & 73.0 & 72.1 & 73.4 & 72.2 & 72.7 \\
\hline $\mathrm{S}_{0} \rightarrow \mathrm{S}_{13}$ & 78.2 & 76.2 & 74.5 & 74.5 & 75.2 & 75.7 & 74.2 \\
\hline $\mathrm{S}_{0} \rightarrow \mathrm{S}_{14}$ & 85.6 & 81.2 & 80.9 & 81.5 & 81.7 & 81.7 & 81.4 \\
\hline
\end{tabular}


Table S1G-2. Compound 1 - CASSCF/QD-NEVPT2 composition of the singlet $3.20 \AA$ ground state while varying number of singlet roots. 9o, 10e Active Space - 25 triplet roots, $X$ singlet roots (where $X$ ranged from 15 to 45$)$. Orbitals: bonding $\mathrm{d}\left(\mathrm{x}^{2}-\mathrm{y}^{2}\right) / \mathrm{C}\left(\mathrm{sp}^{2}\right), \mathrm{d}(\mathrm{xy}), \mathrm{d}(\mathrm{xz}), \mathrm{d}(\mathrm{yz}), \mathrm{d}\left(\mathrm{z}^{2}\right)$, $\mathrm{d}\left(\mathrm{x}^{2}-\mathrm{y}^{2}\right) / \mathrm{C}\left(\mathrm{sp}^{2}\right)^{*}, \pi^{*}(1), \pi^{*}(2), \pi^{*}(3)$.

\begin{tabular}{|c|c|c|c|c|c|}
\hline \multicolumn{6}{|c|}{ CI-Vector Weights (\# Triplets/\# Singlets) } \\
\hline \multirow{2}{*}{\multicolumn{2}{|c|}{$\begin{array}{l}\text { Transition } \\
\mathrm{d}(\mathrm{xz})+\text { bonding } \mathrm{d}\left(\mathrm{x}^{2}-\mathrm{y}^{2}\right) / \mathrm{C}\left(\mathrm{sp}^{2}\right) \\
\rightarrow \mathrm{d}\left(\mathrm{x}^{2}-\mathrm{y}^{2}\right) / \mathrm{C}\left(\mathrm{sp}^{2}\right)^{*}+\pi^{*}(1)\end{array}$}} & \multirow{2}{*}{$\begin{array}{c}\text { 25/15 CPCM } \\
0.5994\end{array}$} & \multirow{2}{*}{$\begin{array}{c}\mathbf{2 5 / 1 5} \text { Gas } \\
0.5998\end{array}$} & \multirow{2}{*}{$\begin{array}{c}\mathbf{2 5 / 2 5} \text { Gas } \\
0.6929\end{array}$} & \multirow{2}{*}{$\begin{array}{c}\text { 25/35 Gas } \\
0.7298\end{array}$} \\
\hline & & & & & \\
\hline $\mathrm{d}(\mathrm{xz}) \rightarrow \pi^{*}(1)$ & & 0.1042 & 0.1057 & 0.0796 & 0.0626 \\
\hline $\begin{array}{l}\text { bonding } \mathrm{d}\left(\mathrm{x}^{2}-\mathrm{y}^{2}\right) / \mathrm{C} \\
\rightarrow \mathrm{d}\left(\mathrm{x}^{2}-\mathrm{y}^{2}\right) / \mathrm{C}\left(\mathrm{sp}^{2}\right)^{3}\end{array}$ & & 0.0560 & 0.0567 & 0.0631 & 0.0663 \\
\hline $\mathrm{d}\left(\mathrm{z}^{2}\right)+\mathrm{d}(\mathrm{xz}) \rightarrow \mathrm{C}$ & & 0.0579 & 0.0549 & 0.0195 & 0.0387 \\
\hline \multicolumn{6}{|c|}{ Singlet $\rightarrow$ Singlet Transition Energies $\left(\mathrm{kcal} \mathrm{mol}^{-1}\right)$} \\
\hline Transition & 25/15 СРCМ & 25/15 Gas & & 25/25 Gas & 25/35 Gas \\
\hline $\mathrm{S}_{0} \rightarrow \mathrm{S}_{1}$ & 42.6 & 38.0 & & 40.1 & 39.3 \\
\hline $\mathrm{S}_{0} \rightarrow \mathrm{S}_{2}$ & 47.3 & 42.5 & & 43.7 & 43.2 \\
\hline $\mathrm{S}_{0} \rightarrow \mathrm{S}_{3}$ & 52.3 & 47.3 & & 48.9 & 48.2 \\
\hline $\mathrm{S}_{0} \rightarrow \mathrm{S}_{4}$ & 64.3 & 59.6 & & 62.1 & 61.2 \\
\hline $\mathrm{S}_{0} \rightarrow \mathrm{S}_{5}$ & 64.9 & 60.5 & & 62.5 & 61.8 \\
\hline $\mathrm{S}_{0} \rightarrow \mathrm{S}_{6}$ & 65.4 & 61.7 & & 63.4 & 63.2 \\
\hline $\mathrm{S}_{0} \rightarrow \mathrm{S}_{7}$ & 70.2 & 65.1 & & 67.7 & 68.0 \\
\hline $\mathrm{S}_{0} \rightarrow \mathrm{S}_{8}$ & 71.3 & 66.7 & & 69.2 & 68.4 \\
\hline $\mathrm{S}_{0} \rightarrow \mathrm{S}_{9}$ & 71.5 & 69.4 & & 70.1 & 69.4 \\
\hline $\mathrm{S}_{0} \rightarrow \mathrm{S}_{10}$ & 72.3 & 72.2 & & 70.6 & 71.3 \\
\hline $\mathrm{S}_{0} \rightarrow \mathrm{S}_{11}$ & 73.3 & 73.4 & & 72.0 & 72.0 \\
\hline $\mathrm{S}_{0} \rightarrow \mathrm{S}_{12}$ & 77.0 & 75.0 & & 72.1 & 72.2 \\
\hline $\mathrm{S}_{0} \rightarrow \mathrm{S}_{13}$ & 78.2 & 76.2 & & 74.5 & 75.7 \\
\hline $\mathrm{S}_{0} \rightarrow \mathrm{S}_{14}$ & 85.6 & 81.2 & & 81.5 & 81.7 \\
\hline
\end{tabular}


Table S1G-3. Compound 1 - CASSCF/QD-NEVPT2 composition of the formal Ni(I) ground state while varying number of doublet roots. 8o, 9e Active Space -25 quartet roots, $X$ doublet roots (where $X$ ranged from 15 to 45 ).

\begin{tabular}{|c|c|c|c|c|c|}
\hline \multicolumn{6}{|c|}{ CI-Vector Weights (\# Quartets / \# Doublets) } \\
\hline Transition & 25/15 CPCM & 25/15 Gas & 25/25 Gas & $25 / 35$ Gas & $25 / 45$ Gas \\
\hline $\mathrm{d}(\mathrm{xz}) \rightarrow \pi^{*}(1)$ & 0.7390 & 0.7363 & 0.7382 & 0.7701 & 0.7631 \\
\hline $\mathrm{d}(\mathrm{xz}) \rightarrow \pi^{*}(2)$ & 0.0761 & 0.0755 & 0.0746 & 0.0710 & 0.0719 \\
\hline formal $\mathrm{Ni}(\mathrm{I})$ & 0.0733 & 0.0727 & 0.0724 & 0.0716 & 0.0748 \\
\hline $\mathrm{d}(\mathrm{yz}) \rightarrow \pi^{*}(3)$ & 0.0428 & 0.0455 & 0.0474 & 0.0443 & 0.0467 \\
\hline \multicolumn{6}{|c|}{ Doublet $\rightarrow$ Doublet Transition Energies (kcal mol ${ }^{-1}$ ) } \\
\hline Transition & 25/15 CPCM & 25/15 Gas & 25/25 Gas & 25/35 Gas & 25/45 Gas \\
\hline 1 & 8.3 & 9.4 & 9.4 & 9.6 & 9.8 \\
\hline 2 & 11.9 & 12.7 & 12.7 & 12.9 & 13.1 \\
\hline 3 & 22.5 & 23.0 & 22.3 & 22.5 & 22.7 \\
\hline 4 & 26.5 & 26.6 & 26.6 & 26.9 & 27.0 \\
\hline 5 & 27.6 & 28.2 & 26.8 & 27.2 & 27.5 \\
\hline 6 & 29.4 & 30.3 & 30.4 & 30.1 & 30.1 \\
\hline 7 & 31.0 & 31.0 & 30.6 & 30.6 & 30.8 \\
\hline 8 & 33.7 & 34.3 & 34.3 & 34.5 & 34.9 \\
\hline 9 & 35.2 & 36.0 & 36.2 & 36.4 & 36.7 \\
\hline 10 & 36.5 & 37.7 & 37.4 & 37.7 & 37.9 \\
\hline 11 & 36.7 & 38.1 & 37.6 & 37.7 & 38.0 \\
\hline 12 & 37.8 & 38.3 & 38.0 & 38.4 & 38.8 \\
\hline 13 & 37.9 & 38.4 & 38.3 & 38.6 & 39.0 \\
\hline 14 & 49.3 & 48.1 & 45.7 & 46.1 & 45.9 \\
\hline
\end{tabular}


Table S1H. Compound 1 - CASSCF/QD-NEVPT2. Transitions for the gas phase spectrum. 9o, 10e Active Space - 25 triplet roots, 15 singlet roots. Orbitals (in order for CI vector notation below): $\mathrm{d}(\mathrm{xy})$, bonding $\mathrm{d}\left(\mathrm{x}^{2}-\mathrm{y}^{2}\right) / \mathrm{C}\left(\mathrm{sp}^{2}\right), \mathrm{d}(\mathrm{yz}), \mathrm{d}\left(\mathrm{z}^{2}\right), \mathrm{d}(\mathrm{xz}), \mathrm{d}\left(\mathrm{x}^{2}-\mathrm{y}^{2}\right) / \mathrm{C}\left(\mathrm{sp}^{2}\right)^{*}, \pi^{*}(1), \pi^{*}(2), \pi^{*}(3)$.

\begin{tabular}{|c|c|c|c|c|c|}
\hline Transition & $\Delta \mathbf{E} / \mathbf{n m}$ & $\Delta \mathrm{E} / \mathrm{kcal} \mathrm{mol}^{-1}$ & CI Vector & $f_{\text {osc }}$ & Contribution \\
\hline \multirow[t]{2}{*}{$\mathrm{S}_{0} \rightarrow \mathrm{S}_{1}$} & 752.5 & 38.0 & 222120100 & 0.0006862 & 0.8166 \\
\hline & & & 202122100 & --- & 0.0850 \\
\hline \multirow[t]{2}{*}{$\mathrm{S}_{0} \rightarrow \mathrm{S}_{2}$} & 672.0 & 42.5 & 221220100 & 0.0649528 & 0.4507 \\
\hline & & & 222210100 & --- & 0.2848 \\
\hline \multirow{2}{*}{$\mathrm{S}_{0} \rightarrow \mathrm{S}_{3}$} & 604.1 & 47.3 & 221220100 & 0.1224866 & 0.3511 \\
\hline & & & 222210100 & --- & 0.2756 \\
\hline \multirow[t]{2}{*}{$\mathrm{S}_{0} \rightarrow \mathrm{S}_{4}$} & 479.5 & 59.6 & 222120010 & 0.0003566 & 0.7222 \\
\hline & & & 202122010 & --- & 0.0773 \\
\hline \multirow[t]{2}{*}{$\mathrm{S}_{0} \rightarrow \mathrm{S}_{5}$} & 472.4 & 60.5 & 122220100 & 0.0001327 & 0.7056 \\
\hline & & & 221211100 & --- & 0.0791 \\
\hline \multirow[t]{2}{*}{$\mathrm{S}_{0} \rightarrow \mathrm{S}_{6}$} & 463.2 & 61.7 & 222210010 & 0.0858212 & 0.3931 \\
\hline & & & 221220010 & --- & 0.2037 \\
\hline \multirow[t]{2}{*}{$\mathrm{S}_{0} \rightarrow \mathrm{S}_{7}$} & 439.3 & 65.1 & 222120001 & 0.0000514 & 0.7108 \\
\hline & & & 202122001 & --- & 0.0744 \\
\hline \multirow[t]{2}{*}{$\mathrm{S}_{0} \rightarrow \mathrm{S}_{8}$} & 428.4 & 66.7 & 221220010 & 0.0799110 & 0.3735 \\
\hline & & & 222210001 & --- & 0.3188 \\
\hline \multirow[t]{2}{*}{$\mathrm{S}_{0} \rightarrow \mathrm{S}_{9}$} & 411.9 & 69.4 & 222210001 & 0.1256711 & 0.2387 \\
\hline & & & 221220010 & --- & 0.2186 \\
\hline \multirow[t]{2}{*}{$\mathrm{S}_{0} \rightarrow \mathrm{S}_{10}$} & 396.2 & 72.2 & 222211000 & 0.0001096 & 0.7697 \\
\hline & & & 212212000 & --- & 0.0922 \\
\hline \multirow[t]{2}{*}{$\mathrm{S}_{0} \rightarrow \mathrm{S}_{11}$} & 389.3 & 73.4 & 122221000 & 0.0146636 & 0.6323 \\
\hline & & & 122211100 & --- & 0.0868 \\
\hline \multirow[t]{2}{*}{$\mathrm{S}_{0} \rightarrow \mathrm{S}_{12}$} & 381.1 & 75.0 & 221221000 & 0.0008472 & 0.7203 \\
\hline & & & 211222000 & --- & 0.0560 \\
\hline \multirow[t]{2}{*}{$\mathrm{S}_{0} \rightarrow \mathrm{S}_{13}$} & 375.2 & 76.2 & 222121000 & 0.1025873 & 0.4292 \\
\hline & & & 222210001 & --- & 0.1754 \\
\hline \multirow[t]{2}{*}{$\mathrm{S}_{0} \rightarrow \mathrm{S}_{14}$} & 351.9 & 81.2 & 221220001 & 0.4703139 & 0.5884 \\
\hline & & & 212221000 & --- & 0.0772 \\
\hline
\end{tabular}


Table S1I-1. Compound 1 - CASSCF/QD-NEVPT2. Transitions for the CPCM(THF) phase spectrum. 9o, 10e Active Space - 25 triplet roots, 15 singlet roots. Orbital (in order for CI vector notation below): $\mathrm{d}(\mathrm{xy})$, bonding $\mathrm{d}\left(\mathrm{x}^{2}-\mathrm{y}^{2}\right) / \mathrm{C}\left(\mathrm{sp}^{2}\right), \mathrm{d}(\mathrm{yz}), \mathrm{d}(\mathrm{xz}), \mathrm{d}\left(\mathrm{z}^{2}\right), \mathrm{d}\left(\mathrm{x}^{2}-\mathrm{y}^{2}\right) / \mathrm{C}\left(\mathrm{sp}^{2}\right)^{*}, \pi^{*}(1), \pi^{*}(2)$, $\pi^{*}(3)$.

\begin{tabular}{|c|c|c|c|c|c|}
\hline Transition & $\Delta \mathbf{E} / \mathbf{n m}$ & $\Delta \mathrm{E} / \mathrm{kcal} \mathrm{mol}^{-1}$ & CI Vector & $f_{\text {osc }}$ & Contribution \\
\hline \multirow{2}{*}{$\mathrm{S}_{0} \rightarrow \mathrm{S}_{1}$} & 671.4 & 42.6 & 222210100 & 0.0008408 & 0.8161 \\
\hline & & & 202212100 & --- & 0.0876 \\
\hline \multirow[t]{2}{*}{$\mathrm{S}_{0} \rightarrow \mathrm{S}_{2}$} & 604.2 & 47.3 & 221220100 & 0.0622469 & 0.4193 \\
\hline & & & 222120100 & --- & 0.3382 \\
\hline \multirow[t]{2}{*}{$\mathrm{S}_{0} \rightarrow \mathrm{S}_{3}$} & 546.6 & 52.3 & 221220100 & 0.1520657 & 0.3722 \\
\hline & & & 222120100 & --- & 0.2674 \\
\hline \multirow[t]{2}{*}{$\mathrm{S}_{0} \rightarrow \mathrm{S}_{4}$} & 444.6 & 64.3 & 222120010 & 0.1043472 & 0.5270 \\
\hline & & & 221220010 & --- & 0.1267 \\
\hline \multirow{2}{*}{$\mathrm{S}_{0} \rightarrow \mathrm{S}_{5}$} & 440.4 & 64.9 & 122220100 & 0.0001963 & 0.4135 \\
\hline & 470.4 & 04.9 & 222210010 & --- & 0.3424 \\
\hline \multirow[t]{2}{*}{$\mathrm{S}_{0} \rightarrow \mathrm{S}_{6}$} & 437.3 & 65.4 & 222210010 & 0.0003333 & 0.4320 \\
\hline & & & 122220100 & --- & 0.3239 \\
\hline \multirow[t]{2}{*}{$\mathrm{S}_{0} \rightarrow \mathrm{S}_{7}$} & 407.1 & 70.2 & 222121000 & 0.0002537 & 0.6733 \\
\hline & & & 221221000 & --- & 0.1149 \\
\hline \multirow[t]{2}{*}{$\mathrm{S}_{0} \rightarrow \mathrm{S}_{8}$} & 401.3 & 71.3 & 222211000 & 0.0305023 & 0.2808 \\
\hline & & & 221220010 & --- & 0.2155 \\
\hline \multirow[t]{2}{*}{$\mathrm{S}_{0} \rightarrow \mathrm{S}_{9}$} & 399.9 & 71.5 & 122221000 & 0.0329884 & 0.4648 \\
\hline & & & 222211000 & --- & 0.1385 \\
\hline \multirow[t]{2}{*}{$\mathrm{S}_{0} \rightarrow \mathrm{S}_{10}$} & 395.3 & 72.3 & 222210001 & 0.0001302 & 0.7121 \\
\hline & & & 202212001 & --- & 0.0765 \\
\hline \multirow[t]{2}{*}{$\mathrm{S}_{0} \rightarrow \mathrm{S}_{11}$} & 390.1 & 73.3 & 221221000 & 0.0016152 & 0.5853 \\
\hline & & & 222121000 & --- & 0.1189 \\
\hline \multirow[t]{2}{*}{$\mathrm{S}_{0} \rightarrow \mathrm{S}_{12}$} & 371.3 & 77.0 & 221220010 & 0.0139708 & 0.3376 \\
\hline & & & 222211000 & --- & 0.2166 \\
\hline \multirow[t]{2}{*}{$\mathrm{S}_{0} \rightarrow \mathrm{S}_{13}$} & 365.5 & 78.2 & 222120001 & 0.1967238 & 0.4902 \\
\hline & & & 222211000 & --- & 0.0927 \\
\hline \multirow[t]{2}{*}{$\mathrm{S}_{0} \rightarrow \mathrm{S}_{14}$} & 333.9 & 85.6 & 221220001 & 0.4607820 & 0.6454 \\
\hline & & & 212221000 & --- & 0.0665 \\
\hline
\end{tabular}


Table S1I-2. Compound 1 - CASSCF/QD-NEVPT2. Spin-orbit corrected absorption transitions for the $\mathrm{CPCM}(\mathrm{THF})$ phase spectrum. 9o, 10e Active Space -25 triplet roots, 15 singlet roots.

\begin{tabular}{cccccc}
\hline $\begin{array}{c}\text { Energy/ } \\
\mathbf{n m}\end{array}$ & $\begin{array}{c}\text { Energy/ } \\
\text { kcal mol }^{\mathbf{1}}\end{array}$ & $\boldsymbol{f}_{\text {osc }}$ & $\begin{array}{c}\text { Energy/ } \\
\mathbf{n m}\end{array}$ & $\begin{array}{c}\text { Energy/ } \\
\mathbf{k c a l ~ m o l}^{\mathbf{1}}\end{array}$ & $\boldsymbol{f}_{\text {osc }}$ \\
\hline 686.8 & 41.6 & 0.0006638 & 420.9 & 67.9 & 0.0026607 \\
681.3 & 42.0 & 0.0000492 & 420.7 & 68.0 & 0.0000932 \\
679.1 & 42.1 & 0.0014753 & 413.2 & 69.2 & 0.0153295 \\
672.4 & 42.5 & 0.0018573 & 412.5 & 69.3 & 0.0001385 \\
641.0 & 44.6 & 0.0000800 & 412.5 & 69.3 & 0.0028688 \\
640.3 & 44.7 & 0.0022972 & 405.7 & 70.5 & 0.0001127 \\
638.3 & 44.8 & 0.0002073 & 398.7 & 71.7 & 0.0002244 \\
602.4 & 47.5 & 0.0625017 & 398.2 & 71.8 & 0.0400852 \\
567.6 & 50.4 & 0.0000177 & 397.7 & 71.9 & 0.0078340 \\
566.9 & 50.4 & 0.0003588 & 389.3 & 73.4 & 0.0017359 \\
566.5 & 50.5 & 0.0056594 & 381.8 & 74.9 & 0.0000093 \\
548.2 & 52.2 & 0.0000804 & 381.7 & 74.9 & 0.0007565 \\
548.0 & 52.2 & 0.0000025 & 381.7 & 74.9 & 0.0000361 \\
547.4 & 52.2 & 0.0077188 & 378.1 & 75.6 & 0.0000159 \\
541.3 & 52.8 & 0.1271535 & 377.9 & 75.6 & 0.0081108 \\
453.0 & 63.1 & 0.0581791 & 377.2 & 75.8 & 0.0001353 \\
446.5 & 64.0 & 0.0000000 & 369.9 & 77.3 & 0.0213533 \\
446.4 & 64.1 & 0.0013619 & 364.8 & 78.4 & 0.1646061 \\
444.2 & 64.4 & 0.0002830 & 350.5 & 81.6 & 0.0000067 \\
437.0 & 65.4 & 0.0000814 & 350.3 & 81.6 & 0.0222811 \\
434.5 & 65.8 & 0.0442016 & 350.2 & 81.6 & 0.0000361 \\
422.4 & 67.7 & 0.0020976 & 332.2 & 86.1 & 0.4457060 \\
\hline
\end{tabular}


Table S1J. Compound 1 - TDDFT calculated lowest transition energies with CPCM(THF).

\begin{tabular}{|c|c|c|c|c|c|c|c|}
\hline State & Root & Multiplicity & $\Delta E / n m$ & $\Delta \mathrm{E} / \mathrm{kcal} \mathrm{mol}^{-1}$ & Transition & $f_{\text {osc }}$ & Contribution \\
\hline \multirow[t]{2}{*}{0} & 0 & 1 & --- & $\begin{array}{l}-- \\
-\end{array}$ & $\begin{array}{l}-- \\
-\end{array}$ & --- & --- \\
\hline & & & & & --- & --- & --- \\
\hline \multirow[t]{2}{*}{1} & 1 & 3 & 858.5 & 33.3 & $119 a \rightarrow 123 a$ & --- & 0.77795 \\
\hline & & & & & $118 \mathrm{a} \rightarrow 123 \mathrm{a}$ & --- & 0.13713 \\
\hline \multirow[t]{2}{*}{2} & 2 & 3 & 745.4 & 38.4 & $117 a \rightarrow 123 a$ & --- & 0.70862 \\
\hline & & & & & $118 a \rightarrow 123 a$ & --- & 0.10691 \\
\hline \multirow[t]{2}{*}{3} & 5 & 3 & 689.5 & 41.4 & $119 a \rightarrow 120 a$ & --- & 0.86068 \\
\hline & & & & & $118 \mathrm{a} \rightarrow 120 \mathrm{a}$ & --- & 0.09258 \\
\hline \multirow[t]{2}{*}{4} & 3 & 3 & 677.1 & 42.2 & $116 a \rightarrow 123 a$ & --- & 0.76487 \\
\hline & & & & & $115 a \rightarrow 123 a$ & --- & 0.08395 \\
\hline \multirow[t]{2}{*}{5} & 8 & 3 & 660.6 & 43.3 & $118 \mathrm{a} \rightarrow 120 \mathrm{a}$ & --- & 0.74196 \\
\hline & & & & & $117 \mathrm{a} \rightarrow 120 \mathrm{a}$ & --- & 0.11865 \\
\hline \multirow[t]{2}{*}{6} & 6 & 3 & 613.9 & 46.6 & $117 \mathrm{a} \rightarrow 120 \mathrm{a}$ & --- & 0.81309 \\
\hline & & & & & $118 \mathrm{a} \rightarrow 120 \mathrm{a}$ & --- & 0.12652 \\
\hline \multirow[t]{2}{*}{7} & 14 & 3 & 593.8 & 48.1 & $115 \mathrm{a} \rightarrow 120 \mathrm{a}$ & --- & 0.70784 \\
\hline & & & & & $114 \mathrm{a} \rightarrow 120 \mathrm{a}$ & --- & 0.13833 \\
\hline \multirow[t]{2}{*}{8} & 1 & 1 & 580.6 & 49.2 & $119 a \rightarrow 120 a$ & 0.0008793 & 0.85637 \\
\hline & & & & & $118 \mathrm{a} \rightarrow 120 \mathrm{a}$ & --- & 0.08983 \\
\hline \multirow[t]{2}{*}{9} & 4 & 3 & 530.2 & 53.9 & $114 a \rightarrow 123 a$ & --- & 0.48094 \\
\hline & & & & & $109 a \rightarrow 123 a$ & --- & 0.16890 \\
\hline \multirow[t]{2}{*}{10} & 7 & 3 & 504.4 & 56.7 & $116 a \rightarrow 120 a$ & --- & 0.85548 \\
\hline & & & & & $115 \mathrm{a} \rightarrow 120 \mathrm{a}$ & --- & 0.07395 \\
\hline \multirow[t]{2}{*}{11} & 9 & 3 & 504.2 & 56.7 & $114 \mathrm{a} \rightarrow 120 \mathrm{a}$ & --- & 0.76732 \\
\hline & & & & & $115 \mathrm{a} \rightarrow 120 \mathrm{a}$ & --- & 0.16764 \\
\hline \multirow[t]{2}{*}{12} & 2 & 1 & 486.4 & 58.8 & $119 a \rightarrow 123 a$ & 0.0149377 & 0.43727 \\
\hline & & & & & $117 \mathrm{a} \rightarrow 120 \mathrm{a}$ & --- & 0.28572 \\
\hline \multirow[t]{2}{*}{13} & 11 & 1 & 464.3 & 61.6 & $115 \mathrm{a} \rightarrow 120 \mathrm{a}$ & 0.0010048 & 0.74526 \\
\hline & & & & & $114 \mathrm{a} \rightarrow 120 \mathrm{a}$ & --- & 0.17279 \\
\hline \multirow[t]{2}{*}{14} & 10 & 3 & 456.9 & 62.6 & $113 a \rightarrow 120 a$ & --- & 0.81346 \\
\hline & & & & & $114 \mathrm{a} \rightarrow 120 \mathrm{a}$ & --- & 0.04189 \\
\hline \multirow[t]{2}{*}{15} & 3 & 1 & 451.4 & 63.3 & $117 \mathrm{a} \rightarrow 123 \mathrm{a}$ & 0.0000160 & 0.69793 \\
\hline & & & & & $118 a \rightarrow 123 a$ & --- & 0.09923 \\
\hline \multirow[t]{2}{*}{16} & 17 & 3 & 451.4 & 63.3 & $118 \mathrm{a} \rightarrow 121 \mathrm{a}$ & --- & 0.71600 \\
\hline & & & & & $117 \mathrm{a} \rightarrow 121 \mathrm{a}$ & --- & 0.11668 \\
\hline \multirow[t]{2}{*}{17} & 4 & 1 & 438.8 & 65.2 & $117 \mathrm{a} \rightarrow 120 \mathrm{a}$ & 0.0091056 & 0.31383 \\
\hline & & & & & $119 a \rightarrow 123 a$ & --- & 0.20057 \\
\hline \multirow[t]{2}{*}{18} & 5 & 1 & 438.8 & 65.2 & $118 \mathrm{a} \rightarrow 120 \mathrm{a}$ & 0.0000671 & 0.34012 \\
\hline & & & & & $116 a \rightarrow 123 a$ & --- & 0.33024 \\
\hline \multirow[t]{2}{*}{19} & 6 & 1 & 432.3 & 66.1 & $116 a \rightarrow 123 a$ & 0.0006521 & 0.39229 \\
\hline & & & & & $118 \mathrm{a} \rightarrow 120 \mathrm{a}$ & --- & 0.30750 \\
\hline \multirow[t]{2}{*}{20} & 9 & 1 & 429.6 & 66.5 & $114 \mathrm{a} \rightarrow 120 \mathrm{a}$ & 0.0002344 & 0.74666 \\
\hline & & & & & $115 \mathrm{a} \rightarrow 120 \mathrm{a}$ & --- & 0.16061 \\
\hline
\end{tabular}


Table S1K. Compound 1 - TDDFT with CPCM(THF). Transitions corresponding to singlets down to $300 \mathrm{~nm}$.

\begin{tabular}{|c|c|c|c|c|c|}
\hline Root & $\Delta E / n m$ & $\Delta \mathrm{E} / \mathrm{kcal} \mathrm{mol}^{-1}$ & Transition & $f_{\text {osc }}$ & Contribution \\
\hline \multirow[t]{2}{*}{1} & 580.6 & 49.2 & $119 \mathrm{a} \rightarrow 120 \mathrm{a}$ & 0.0008793 & 0.85637 \\
\hline & & & $118 \mathrm{a} \rightarrow 120 \mathrm{a}$ & --- & 0.08983 \\
\hline \multirow[t]{2}{*}{2} & 486.4 & 58.8 & $119 a \rightarrow 123 a$ & 0.0149377 & 0.43727 \\
\hline & & & $117 \mathrm{a} \rightarrow 120 \mathrm{a}$ & --- & 0.28572 \\
\hline \multirow[t]{2}{*}{11} & 464.3 & 61.6 & $115 \mathrm{a} \rightarrow 120 \mathrm{a}$ & 0.0010048 & 0.74526 \\
\hline & & & $114 \mathrm{a} \rightarrow 120 \mathrm{a}$ & --- & 0.17279 \\
\hline \multirow[t]{2}{*}{3} & 451.4 & 63.3 & $117 \mathrm{a} \rightarrow 123 \mathrm{a}$ & 0.0000160 & 0.69793 \\
\hline & & & $118 \mathrm{a} \rightarrow 123 \mathrm{a}$ & --- & 0.09923 \\
\hline \multirow[t]{2}{*}{4} & 438.8 & 65.2 & $117 \mathrm{a} \rightarrow 120 \mathrm{a}$ & 0.0091056 & 0.31383 \\
\hline & & & $119 a \rightarrow 123 a$ & --- & 0.20057 \\
\hline \multirow[t]{2}{*}{5} & 438.8 & 65.2 & $118 \mathrm{a} \rightarrow 120 \mathrm{a}$ & 0.0000671 & 0.34012 \\
\hline & & & $116 a \rightarrow 123 a$ & --- & 0.33024 \\
\hline \multirow[t]{2}{*}{6} & 432.3 & 66.1 & $116 a \rightarrow 123 a$ & 0.0006521 & 0.39229 \\
\hline & & & $118 \mathrm{a} \rightarrow 120 \mathrm{a}$ & --- & 0.30750 \\
\hline \multirow[t]{2}{*}{9} & 429.6 & 66.5 & $114 \mathrm{a} \rightarrow 120 \mathrm{a}$ & 0.0002344 & 0.74666 \\
\hline & & & $115 \mathrm{a} \rightarrow 120 \mathrm{a}$ & --- & 0.16061 \\
\hline \multirow[t]{2}{*}{8} & 429.3 & 66.6 & $116 a \rightarrow 120 a$ & 0.0774053 & 0.73096 \\
\hline & & & $117 \mathrm{a} \rightarrow 120 \mathrm{a}$ & --- & 0.11234 \\
\hline \multirow[t]{2}{*}{7} & 416.4 & 68.7 & $114 a \rightarrow 123 a$ & 0.0040780 & 0.43475 \\
\hline & & & $109 a \rightarrow 123 a$ & --- & 0.10090 \\
\hline \multirow[t]{2}{*}{10} & 411.7 & 69.4 & $119 a \rightarrow 121 a$ & 0.0002556 & 0.90253 \\
\hline & & & $118 \mathrm{a} \rightarrow 121 \mathrm{a}$ & --- & 0.06081 \\
\hline \multirow[t]{2}{*}{14} & 393.7 & 72.6 & $118 \mathrm{a} \rightarrow 121 \mathrm{a}$ & 0.0002484 & 0.74852 \\
\hline & & & $117 \mathrm{a} \rightarrow 121 \mathrm{a}$ & --- & 0.13455 \\
\hline \multirow[t]{2}{*}{13} & 374.9 & 76.3 & $119 a \rightarrow 122 a$ & 0.0000090 & 0.88873 \\
\hline & & & $118 \mathrm{a} \rightarrow 122 \mathrm{a}$ & --- & 0.07966 \\
\hline \multirow[t]{2}{*}{12} & 372.2 & 76.8 & $117 \mathrm{a} \rightarrow 121 \mathrm{a}$ & 0.0103918 & 0.75836 \\
\hline & & & $118 \mathrm{a} \rightarrow 121 \mathrm{a}$ & --- & 0.13533 \\
\hline \multirow[t]{2}{*}{18} & 363.5 & 78.7 & $118 a \rightarrow 122 a$ & 0.0002226 & 0.72376 \\
\hline & & & $117 \mathrm{a} \rightarrow 122 \mathrm{a}$ & --- & 0.11295 \\
\hline \multirow[t]{2}{*}{21} & 343.8 & 83.2 & $115 \mathrm{a} \rightarrow 121 \mathrm{a}$ & 0.0293580 & 0.61427 \\
\hline & & & $114 \mathrm{a} \rightarrow 121 \mathrm{a}$ & --- & 0.22459 \\
\hline \multirow[t]{2}{*}{17} & 341.1 & 83.8 & $116 a \rightarrow 121 a$ & 0.0253815 & 0.72971 \\
\hline & & & $117 \mathrm{a} \rightarrow 122 \mathrm{a}$ & --- & 0.10940 \\
\hline \multirow[t]{2}{*}{16} & 339.9 & 84.1 & $112 a \rightarrow 120 a$ & 0.0000921 & 0.96045 \\
\hline & & & --- & --- & --- \\
\hline \multirow[t]{2}{*}{15} & 335.0 & 85.4 & $117 a \rightarrow 122 a$ & 0.0228657 & 0.61108 \\
\hline & & & $116 a \rightarrow 121 a$ & --- & 0.12381 \\
\hline \multirow[t]{2}{*}{19} & 334.8 & 85.4 & $114 a \rightarrow 121 a$ & 0.0004394 & 0.68195 \\
\hline & & & $115 \mathrm{a} \rightarrow 121 \mathrm{a}$ & --- & 0.24704 \\
\hline \multirow[t]{2}{*}{25} & 321.7 & 88.9 & $115 \mathrm{a} \rightarrow 122 \mathrm{a}$ & 0.0021238 & 0.61609 \\
\hline & & & $114 a \rightarrow 122 a$ & --- & 0.16497 \\
\hline
\end{tabular}


Table S1L. Compound 1 with Ni-C distance of $3.20 \AA-$ CASSCF/QD-NEVPT2 lowest transition energies in CPCM(THF). 80, 10e Active Space -25 triplet roots, 15 singlet roots. Orbitals (in order for CI vector notation below): $\mathrm{d}(\mathrm{xy}), \mathrm{d}(\mathrm{yz}), \mathrm{d}\left(\mathrm{z}^{2}\right), \mathrm{d}(\mathrm{xz})$, bonding $\mathrm{d}\left(\mathrm{x}^{2}-\mathrm{y}^{2}\right) / \mathrm{C}\left(\mathrm{sp}^{2}\right), \mathrm{d}\left(\mathrm{x}^{2}-\right.$ $\left.\mathrm{y}^{2}\right) / \mathrm{C}\left(\mathrm{sp}^{2}\right)^{*}, \pi^{*}(1), \pi^{*}(2)$.

\begin{tabular}{|c|c|c|c|c|c|c|c|}
\hline State & Root & Multiplicity & $\Delta E / n m$ & $\Delta \mathrm{E} / \mathrm{kcal}^{\mathrm{mol}}{ }^{-1}$ & CI Vector & $f_{\text {osc }}$ & Contribution \\
\hline \multirow[t]{3}{*}{0} & 0 & 1 & --- & --- & 22211110 & --- & 0.5994 \\
\hline & & & & & 22212010 & --- & 0.1042 \\
\hline & & & & & 22112110 & --- & 0.0579 \\
\hline \multirow[t]{2}{*}{1} & 0 & 3 & 9220.8 & 3.1 & 22211110 & --- & 0.7413 \\
\hline & & & & & 22112110 & --- & 0.0631 \\
\hline \multirow[t]{2}{*}{2} & 1 & 3 & 4238.2 & 6.8 & 22121110 & --- & 0.8175 \\
\hline & & & & & 22122010 & --- & 0.1200 \\
\hline \multirow[t]{2}{*}{3} & 1 & 1 & 3665.3 & 7.8 & 22121110 & 0.0000056 & 0.8154 \\
\hline & & & & & 22122010 & --- & 0.1208 \\
\hline \multirow[t]{2}{*}{4} & 2 & 3 & 3436.2 & 8.3 & 22211110 & -- & 0.7508 \\
\hline & & & & & 22212010 & --- & 0.0904 \\
\hline \multirow[t]{2}{*}{5} & 3 & 3 & 2779.3 & 10.3 & 22121110 & -- & 0.9893 \\
\hline & & & & & 12221110 & --- & 0.0030 \\
\hline \multirow[t]{2}{*}{6} & 4 & 3 & 2465.5 & 11.6 & 21221110 & --- & 0.7577 \\
\hline & & & & & 21222010 & --- & 0.1076 \\
\hline \multirow[t]{2}{*}{7} & 2 & 1 & 2439.5 & 11.7 & 21221110 & 0.0000012 & 0.6968 \\
\hline & & & & & 21222010 & --- & 0.1037 \\
\hline \multirow[t]{2}{*}{8} & 5 & 3 & 2034.0 & 14.1 & 21221110 & -- & 0.8601 \\
\hline & & & & & 22211110 & --- & 0.0519 \\
\hline \multirow[t]{2}{*}{9} & 3 & 1 & 1185.6 & 24.1 & 22220110 & 0.0001996 & 0.7437 \\
\hline & & & & & 22221010 & --- & 0.0721 \\
\hline \multirow[t]{2}{*}{10} & 6 & 3 & 1184.1 & 24.1 & 22220110 & --- & 0.7898 \\
\hline & & & & & 22221010 & --- & 0.0749 \\
\hline \multirow[t]{2}{*}{11} & 4 & 1 & 1117.7 & 25.6 & 22211101 & 0.0501900 & 0.6199 \\
\hline & & & & & 22212001 & --- & 0.0954 \\
\hline \multirow[t]{2}{*}{12} & 5 & 1 & 1102.6 & 25.9 & 21212110 & 0.0046113 & 0.3668 \\
\hline & & & & & 12221110 & --- & 0.3589 \\
\hline \multirow[t]{2}{*}{13} & 7 & 3 & 1070.5 & 26.7 & 12221110 & --- & 0.4548 \\
\hline & & & & & 21212110 & --- & 0.4330 \\
\hline \multirow[t]{2}{*}{14} & 8 & 3 & 1050.9 & 27.2 & 22121101 & --- & 0.8032 \\
\hline & & & & & 22122001 & --- & 0.1181 \\
\hline \multirow[t]{2}{*}{15} & 9 & 3 & 1043.3 & 27.4 & 22211101 & --- & 0.7337 \\
\hline & & & & & 22212001 & --- & 0.0821 \\
\hline \multirow[t]{2}{*}{16} & 6 & 1 & 1042.0 & 27.4 & 22121101 & 0.0000466 & 0.8118 \\
\hline & & & & & 22122001 & --- & 0.1196 \\
\hline \multirow[t]{2}{*}{17} & 10 & 3 & 1008.1 & 28.4 & 12221110 & --- & 0.4842 \\
\hline & & & & & 21212110 & --- & 0.4145 \\
\hline \multirow[t]{2}{*}{18} & 11 & 3 & 994.2 & 28.8 & 22211101 & --- & 0.8211 \\
\hline & & & & & 22112101 & --- & 0.0703 \\
\hline \multirow[t]{2}{*}{19} & 12 & 3 & 960.4 & 29.8 & 22121101 & --- & 0.9873 \\
\hline & & & & & 12221101 & --- & 0.0030 \\
\hline \multirow[t]{2}{*}{20} & 7 & 1 & 896.2 & 31.9 & 21221101 & 0.0028482 & 0.7468 \\
\hline & & & & & 21222001 & --- & 0.1085 \\
\hline
\end{tabular}


Table S1M. Compound 1 - CASSCF/QD-NEVPT2 composition of the singlet equilibrium ground state with Ni-C distance of $3.20 \AA-C A S S C F / Q D-N E V P T 2$ in gas phase. 8o, 10e Active Space 25 triplet roots, 15 singlet roots. Orbitals (in order for CI vector notation below): $d(x y), d(y z)$, $\mathrm{d}\left(\mathrm{z}^{2}\right), \mathrm{d}(\mathrm{xz})$, bonding $\mathrm{d}\left(\mathrm{x}^{2}-\mathrm{y}^{2}\right) / \mathrm{C}\left(\mathrm{sp}^{2}\right), \mathrm{d}\left(\mathrm{x}^{2}-\mathrm{y}^{2}\right) / \mathrm{C}\left(\mathrm{sp}^{2}\right)^{*}, \pi^{*}(1), \pi^{*}(2)$.

\begin{tabular}{llc}
\hline CI Vector & Transition & Contribution \\
\hline 22211110 & $\mathrm{~d}(\mathrm{xz})+$ bonding $\mathrm{d}\left(\mathrm{x}^{2}-\mathrm{y}^{2}\right) / \mathrm{C}\left(\mathrm{sp}^{2}\right) \rightarrow \mathrm{d}\left(\mathrm{x}^{2}-\mathrm{y}^{2}\right) / \mathrm{C}\left(\mathrm{sp}^{2}\right)^{*}+\pi^{*}(1)$ & 0.5998 \\
22212010 & $\mathrm{~d}(\mathrm{xz}) \rightarrow \pi^{*}(1)$ & 0.1057 \\
22221100 & bonding $\mathrm{d}\left(\mathrm{x}^{2}-\mathrm{y}^{2}\right) / \mathrm{C}\left(\mathrm{sp}^{2}\right) \rightarrow \mathrm{d}\left(\mathrm{x}^{2}-\mathrm{y}^{2}\right) / \mathrm{C}\left(\mathrm{sp}^{2}\right)^{*}$ & 0.0567 \\
22112110 & $\mathrm{~d}(\mathrm{xz})+\mathrm{d}\left(\mathrm{z}^{2}\right) \rightarrow \mathrm{d}\left(\mathrm{x}^{2}-\mathrm{y}^{2}\right) / \mathrm{C}\left(\mathrm{sp}^{2}\right)^{*}+\pi^{*}(1)$ & 0.0549 \\
22210210 & $\mathrm{~d}(\mathrm{xz})+2 \mathrm{x}\left[\right.$ bonding d$\left.\left(\mathrm{x}^{2}-\mathrm{y}^{2}\right) / \mathrm{C}\left(\mathrm{sp}^{2}\right)\right] \rightarrow 2 \mathrm{x}\left[\mathrm{d}\left(\mathrm{x}^{2}-\mathrm{y}^{2}\right) / \mathrm{C}\left(\mathrm{sp}^{2}\right)^{*}\right]+\pi^{*}(1)$ & 0.0472 \\
21221110 & $\mathrm{~d}(\mathrm{yz})+$ bonding d$\left(\mathrm{x}^{2}-\mathrm{y}^{2}\right) / C\left(\mathrm{sp}^{2}\right) \rightarrow \mathrm{d}\left(\mathrm{x}^{2}-\mathrm{y}^{2}\right) / \mathrm{C}\left(\mathrm{sp}^{2}\right)^{*}+\pi^{*}(1)$ & 0.0434 \\
22211101 & $\mathrm{~d}(\mathrm{xz})+$ bonding d$\left(\mathrm{x}^{2}-\mathrm{y}^{2}\right) / \mathrm{C}\left(\mathrm{sp}^{2}\right) \rightarrow \mathrm{d}\left(\mathrm{x}^{2}-\mathrm{y}^{2}\right) / \mathrm{C}\left(\mathrm{sp}^{2}\right)^{*}+\pi^{*}(2)$ & 0.0273 \\
11222110 & $\mathrm{~d}(\mathrm{yz})+\mathrm{d}(\mathrm{xy}) \rightarrow \mathrm{d}\left(\mathrm{x}^{2}-\mathrm{y}^{2}\right) / \mathrm{C}\left(\mathrm{sp}^{2}\right)^{*}+\pi^{*}(1)$ & 0.0170 \\
22222000 & $\mathrm{Closed}$ shell singlet $(\mathrm{CSS}) \mathrm{d}^{8}$ & 0.0105 \\
21222010 & $\mathrm{~d}(\mathrm{yz}) \rightarrow \pi^{*}(1)$ & 0.0067 \\
22212001 & $\mathrm{~d}(\mathrm{xz}) \rightarrow \pi^{*}(2)$ & 0.0047 \\
22122100 & $\mathrm{~d}\left(\mathrm{z}^{2}\right) \rightarrow \mathrm{d}\left(\mathrm{x}^{2}-\mathrm{y}^{2}\right) / \mathrm{C}\left(\mathrm{sp}^{2}\right)^{*}$ & 0.0034 \\
21220210 & $2 \mathrm{x}\left[\mathrm{bonding} \mathrm{d}\left(\mathrm{x}^{2}-\mathrm{y}^{2}\right) / \mathrm{C}\left(\mathrm{sp}^{2}\right)\right]+\mathrm{d}(\mathrm{yz}) \rightarrow 2 \mathrm{x}\left[\mathrm{d}\left(\mathrm{x}^{2}-\mathrm{y}^{2}\right) / \mathrm{C}\left(\mathrm{sp}^{2}\right)^{*}\right]+\pi^{*}(1)$ & 0.0034 \\
12212110 & $\mathrm{~d}(\mathrm{xy})+\mathrm{d}(\mathrm{xz}) \rightarrow \mathrm{d}\left(\mathrm{x}^{2}-\mathrm{y}^{2}\right) / \mathrm{C}\left(\mathrm{sp}^{2}\right)^{*}+\pi^{*}(1)$ & 0.0032 \\
\hline
\end{tabular}

Table S1N. Compound 1 - CASSCF/QD-NEVPT2 composition of the singlet equilibrium ground state with Ni-C distance of $3.20 \AA-$ CASSCF/QD-NEVPT2 with CPCM(THF). 8o, 10e Active Space - 25 triplet roots, 15 singlet roots. Orbitals (in order for CI vector notation below): $d(x y)$, $\mathrm{d}(\mathrm{yz}), \mathrm{d}\left(\mathrm{z}^{2}\right), \mathrm{d}(\mathrm{xz})$, bonding $\mathrm{d}\left(\mathrm{x}^{2}-\mathrm{y}^{2}\right) / \mathrm{C}\left(\mathrm{sp}^{2}\right), \mathrm{d}\left(\mathrm{x}^{2}-\mathrm{y}^{2}\right) / \mathrm{C}\left(\mathrm{sp}^{2}\right)^{*}, \pi^{*}(1), \pi^{*}(2)$.

\begin{tabular}{llc}
\hline CI Vector & Transition & Contribution \\
\hline 22211110 & $\mathrm{~d}(\mathrm{xz})+$ bonding $\mathrm{d}\left(\mathrm{x}^{2}-\mathrm{y}^{2}\right) / \mathrm{C}\left(\mathrm{sp}^{2}\right) \rightarrow \mathrm{d}\left(\mathrm{x}^{2}-\mathrm{y}^{2}\right) / \mathrm{C}\left(\mathrm{sp}^{2}\right)^{*}+\pi^{*}(1)$ & 0.5994 \\
22212010 & $\mathrm{~d}(\mathrm{xz}) \rightarrow \pi^{*}(1)$ & 0.1042 \\
22112110 & $\mathrm{~d}(\mathrm{xz})+\mathrm{d}\left(\mathrm{z}^{2}\right) \rightarrow \mathrm{d}\left(\mathrm{x}^{2}-\mathrm{y}^{2}\right) / \mathrm{C}\left(\mathrm{sp}^{2}\right)^{*}+\pi^{*}(1)$ & 0.0579 \\
22221100 & bonding $\mathrm{d}\left(\mathrm{x}^{2}-\mathrm{y}^{2}\right) / \mathrm{C}\left(\mathrm{sp}^{2}\right) \rightarrow \mathrm{d}\left(\mathrm{x}^{2}-\mathrm{y}^{2}\right) / C\left(\mathrm{sp}^{2}\right)^{*}$ & 0.0560 \\
21221110 & $\mathrm{~d}(\mathrm{yz})+$ bonding $\mathrm{d}\left(\mathrm{x}^{2}-\mathrm{y}^{2}\right) / \mathrm{C}\left(\mathrm{sp}^{2}\right) \rightarrow \mathrm{d}\left(\mathrm{x}^{2}-\mathrm{y}^{2}\right) / \mathrm{C}\left(\mathrm{sp}^{2}\right)^{*}+\pi^{*}(1)$ & 0.0463 \\
22210210 & $\mathrm{~d}(\mathrm{xz})+2 \mathrm{x}\left[\right.$ bonding $\left.\mathrm{d}\left(\mathrm{x}^{2}-\mathrm{y}^{2}\right) / \mathrm{C}\left(\mathrm{sp}^{2}\right)\right] \rightarrow 2 \mathrm{x}\left[\mathrm{d}\left(\mathrm{x}^{2}-\mathrm{y}^{2}\right) / \mathrm{C}\left(\mathrm{sp}^{2}\right)^{*}\right]+\pi^{*}(1)$ & 0.0463 \\
22211101 & $\mathrm{~d}(\mathrm{xz})+$ bonding $\mathrm{d}\left(\mathrm{x}^{2}-\mathrm{y}^{2}\right) / \mathrm{C}\left(\mathrm{sp}^{2}\right) \rightarrow \mathrm{d}\left(\mathrm{x}^{2}-\mathrm{y}^{2}\right) / \mathrm{C}\left(\mathrm{sp}^{2}\right)^{*}+\pi^{*}(2)$ & 0.0254 \\
11222110 & $\mathrm{~d}(\mathrm{yz})+\mathrm{d}(\mathrm{xy}) \rightarrow \mathrm{d}\left(\mathrm{x}^{2}-\mathrm{y}^{2}\right) / \mathrm{C}\left(\mathrm{sp}^{2}\right)^{*}+\pi^{*}(1)$ & 0.0169 \\
22222000 & $\mathrm{Closed}$ shell singlet $(\mathrm{CSS}) \mathrm{d}^{8}$ & 0.0102 \\
21222010 & $\mathrm{~d}(\mathrm{yz}) \rightarrow \pi^{*}(1)$ & 0.0071 \\
22212001 & $\mathrm{~d}(\mathrm{xz}) \rightarrow \pi^{*}(2)$ & 0.0043 \\
22122100 & $\mathrm{~d}\left(\mathrm{z}^{2}\right) \rightarrow \mathrm{d}\left(\mathrm{x}^{2}-\mathrm{y}^{2}\right) / \mathrm{C}\left(\mathrm{sp}^{2}\right)^{*}$ & 0.0035 \\
21220210 & $2 \mathrm{x}\left[\right.$ bonding $\left.\mathrm{d}\left(\mathrm{x}^{2}-\mathrm{y}^{2}\right) / \mathrm{C}\left(\mathrm{sp}^{2}\right)\right]+\mathrm{d}(\mathrm{yz}) \rightarrow 2 \mathrm{x}\left[\mathrm{d}\left(\mathrm{x}^{2}-\mathrm{y}^{2}\right) / \mathrm{C}\left(\mathrm{sp}^{2}\right)^{*}\right]+\pi^{*}(1)$ & 0.0035 \\
12212110 & $\mathrm{~d}(\mathrm{xy})+\mathrm{d}(\mathrm{xz}) \rightarrow \mathrm{d}\left(\mathrm{x}^{2}-\mathrm{y}^{2}\right) / \mathrm{C}\left(\mathrm{sp}^{2}\right)^{*}+\pi^{*}(1)$ & 0.0029 \\
\hline
\end{tabular}


Table S1O. Leadings terms of CI-vector for $\mathrm{T}_{0}$ (first triplet manifold reached from vertical excitation of singlet ground state) of 1 in Figure 4. In the vicinity of the equilibrium geometry, the CI-vector is dominated by ${ }^{3} \mathrm{~d}-\mathrm{d}$ transitions. Note that using the relaxed, DFT optimized $T_{\mathrm{d}}$ triplet geometries of 1 (surfaces shown in Figure 1) the ground state triplet CI-vector is very heavily dominated by the $\left[\mathrm{d}\left(\mathrm{x}^{2}-\mathrm{y}^{2}\right)\right]^{1}\left[\mathrm{~d}\left(\mathrm{z}^{2}\right) / \mathrm{C}\left(\mathrm{sp}^{2}\right)^{*}\right]^{1}{ }^{3} \mathrm{~d}-\mathrm{d}$ configuration $(65 \%$ at the $1.9 \AA$ equilibrium geometry, Figure $\mathrm{S} 1 \mathrm{H})$. For $\mathrm{T}_{0}$, at long $\mathrm{Ni}-\mathrm{C}$ distances, the transition $\mathrm{d}(\mathrm{xz}) \rightarrow \pi^{*}(1)$ is very dominant, but still less so than in the repulsive manifold shown in black, and assigned to the aforementioned transition, in Figure 4.

\begin{tabular}{|c|c|c|}
\hline Ni-C Distance $(\AA)$ & Weight & Transition \\
\hline \multirow[t]{2}{*}{1.60} & 0.8420 & $\mathrm{~d}\left(\mathrm{z}^{2}\right) \rightarrow \pi^{*}(1)$ \\
\hline & 0.0669 & $\mathrm{~d}(\mathrm{xy}) \rightarrow \pi^{*}(1)$ \\
\hline \multirow[t]{2}{*}{1.80} & 0.7339 & $\mathrm{~d}\left(\mathrm{z}^{2}\right) \rightarrow \pi^{*}(1)$ \\
\hline & 0.0867 & $\mathrm{~d}(\mathrm{xy}) \rightarrow \pi^{*}(1)$ \\
\hline \multirow[t]{2}{*}{1.89 (equil.) } & 0.4035 & $\mathrm{~d}\left(\mathrm{z}^{2}\right) \rightarrow \mathrm{d}\left(\mathrm{x}^{2}-\mathrm{y}^{2}\right) / \mathrm{C}\left(\mathrm{sp}^{2}\right)^{*}$ \\
\hline & 0.1917 & $\mathrm{~d}(\mathrm{xz}) \rightarrow \pi^{*}(1)$ \\
\hline \multirow[t]{2}{*}{2.20} & 0.4499 & $\mathrm{~d}(\mathrm{xz}) \rightarrow \mathrm{d}\left(\mathrm{x}^{2}-\mathrm{y}^{2}\right) / \mathrm{C}\left(\mathrm{sp}^{2}\right)^{*}$ \\
\hline & 0.1777 & $\mathrm{~d}(\mathrm{yz}) \rightarrow \mathrm{d}\left(\mathrm{x}^{2}-\mathrm{y}^{2}\right) / \mathrm{C}\left(\mathrm{sp}^{2}\right)^{*}$ \\
\hline \multirow[t]{2}{*}{2.40} & 0.5278 & $\mathrm{~d}\left(\mathrm{z}^{2}\right) \rightarrow \mathrm{d}\left(\mathrm{x}^{2}-\mathrm{y}^{2}\right) / \mathrm{C}\left(\mathrm{sp}^{2}\right)^{*}$ \\
\hline & 0.2942 & $\mathrm{~d}\left(\mathrm{z}^{2}\right)+$ bonding $\mathrm{d}\left(\mathrm{x}^{2}-\mathrm{y}^{2}\right) / \mathrm{C}\left(\mathrm{sp}^{2}\right) \rightarrow 2 \mathrm{x}\left[\mathrm{d}\left(\mathrm{x}^{2}-\mathrm{y}^{2}\right) / \mathrm{C}\left(\mathrm{sp}^{2}\right)^{*}\right]$ \\
\hline \multirow[t]{2}{*}{2.60} & 0.4220 & $\mathrm{dz} 2 \rightarrow \pi^{*}(1)$ \\
\hline & 0.4045 & $\mathrm{~d}\left(\mathrm{z}^{2}\right)+$ bonding $\mathrm{d}\left(\mathrm{x}^{2}-\mathrm{y}^{2}\right) / \mathrm{C}\left(\mathrm{sp}^{2}\right) \rightarrow \mathrm{d}\left(\mathrm{x}^{2}-\mathrm{y}^{2}\right) / \mathrm{C}\left(\mathrm{sp}^{2}\right)^{*}+\pi^{*}(1)$ \\
\hline \multirow[t]{2}{*}{2.80} & 0.5427 & $\mathrm{~d}(\mathrm{xz})+$ bonding $\mathrm{d}\left(\mathrm{x}^{2}-\mathrm{y}^{2}\right) / \mathrm{C}\left(\mathrm{sp}^{2}\right) \rightarrow \mathrm{d}\left(\mathrm{x}^{2}-\mathrm{y}^{2}\right) / \mathrm{C}\left(\mathrm{sp}^{2}\right)^{*}+\pi^{*}(1)$ \\
\hline & 0.2368 & $\mathrm{~d}(\mathrm{xz}) \rightarrow \pi^{*}(1)$ \\
\hline \multirow[t]{2}{*}{3.00} & 0.6998 & $\mathrm{~d}(\mathrm{xz})+$ bonding $\mathrm{d}\left(\mathrm{x}^{2}-\mathrm{y}^{2}\right) / \mathrm{C}\left(\mathrm{sp}^{2}\right) \rightarrow \mathrm{d}\left(\mathrm{x}^{2}-\mathrm{y}^{2}\right) / \mathrm{C}\left(\mathrm{sp}^{2}\right)^{*}+\pi^{*}(1)$ \\
\hline & 0.0650 & $\mathrm{~d}(\mathrm{xz}) \rightarrow \pi^{*}(1)$ \\
\hline \multirow[t]{2}{*}{3.20} & 0.7446 & $\mathrm{~d}(\mathrm{xz})+$ bonding $\mathrm{d}\left(\mathrm{x}^{2}-\mathrm{y}^{2}\right) / \mathrm{C}\left(\mathrm{sp}^{2}\right) \rightarrow \mathrm{d}\left(\mathrm{x}^{2}-\mathrm{y}^{2}\right) / \mathrm{C}\left(\mathrm{sp}^{2}\right)^{*}+\pi^{*}(1)$ \\
\hline & 0.0600 & $\mathrm{~d}\left(\mathrm{z}^{2}\right)+$ bonding $\mathrm{d}\left(\mathrm{x}^{2}-\mathrm{y}^{2}\right) / \mathrm{C}\left(\mathrm{sp}^{2}\right) \rightarrow \mathrm{d}\left(\mathrm{x}^{2}-\mathrm{y}^{2}\right) / \mathrm{C}\left(\mathrm{sp}^{2}\right)^{*}+\pi^{*}(1)$ \\
\hline \multirow[t]{2}{*}{3.40} & 0.7572 & $\mathrm{~d}(\mathrm{xz})+$ bonding $\mathrm{d}\left(\mathrm{x}^{2}-\mathrm{y}^{2}\right) / \mathrm{C}\left(\mathrm{sp}^{2}\right) \rightarrow \mathrm{d}\left(\mathrm{x}^{2}-\mathrm{y}^{2}\right) / \mathrm{C}\left(\mathrm{sp}^{2}\right)^{*}+\pi^{*}(1)$ \\
\hline & 0.0610 & bonding $\mathrm{d}\left(\mathrm{x}^{2}-\mathrm{y}^{2}\right) / \mathrm{C}\left(\mathrm{sp}^{2}\right) \rightarrow \mathrm{d}\left(\mathrm{x}^{2}-\mathrm{y}^{2}\right) / \mathrm{C}\left(\mathrm{sp}^{2}\right)^{*}$ \\
\hline \multirow[t]{2}{*}{3.60} & 0.7627 & $\mathrm{~d}(\mathrm{xz})+$ bonding $\mathrm{d}\left(\mathrm{x}^{2}-\mathrm{y}^{2}\right) / \mathrm{C}\left(\mathrm{sp}^{2}\right) \rightarrow \mathrm{d}\left(\mathrm{x}^{2}-\mathrm{y}^{2}\right) / \mathrm{C}\left(\mathrm{sp}^{2}\right)^{*}+\pi^{*}(1)$ \\
\hline & 0.0617 & bonding $\mathrm{d}\left(\mathrm{x}^{2}-\mathrm{y}^{2}\right) / \mathrm{C}\left(\mathrm{sp}^{2}\right) \rightarrow \mathrm{d}\left(\mathrm{x}^{2}-\mathrm{y}^{2}\right) / \mathrm{C}\left(\mathrm{sp}^{2}\right)^{*}$ \\
\hline
\end{tabular}


Table S1P. Spin Contamination Values From BSS Calculation and Yamaguchi Correction ${ }^{1,2}$ BSS Energy of 1. Using $<\mathrm{S}^{2}>$ values and energies for the triplet and BSS solutions for the same geometry, $\left.\left.\alpha=\left\langle\mathrm{S}^{2}\right\rangle_{\text {trip. }} /\left(\left\langle\mathrm{S}^{2}\right\rangle_{\text {trip. }}-\mathrm{S}^{2}\right\rangle_{\mathrm{BSS}}\right), \beta=\left\langle\mathrm{S}^{2}\right\rangle_{\mathrm{BSS}} /\left(\left\langle\mathrm{S}^{2}\right\rangle_{\text {trip. }}-\mathrm{S}^{2}\right\rangle_{\mathrm{BSS}}\right)$, and $\mathrm{E}_{\text {Yamaguchi }}=$ $\alpha \mathrm{E}_{\text {BSS }}-\beta \mathrm{E}_{\text {trip }}$.

\begin{tabular}{|c|c|c|c|c|c|c|c|}
\hline $\begin{array}{l}\text { Ni-C } \\
\text { Dist. } \\
(\AA \AA)\end{array}$ & $\begin{array}{l}<\mathbf{S}^{2}> \\
\mathbf{S}=\mathbf{1}\end{array}$ & $\begin{array}{l}<\mathrm{S}^{2}> \\
\mathrm{BSS}\end{array}$ & $\alpha$ & $\boldsymbol{\beta}$ & $\begin{array}{c}\text { Energy } \\
\mathrm{S}=1 / \\
{\text { kcal } \mathrm{mol}^{-1}}^{-1}\end{array}$ & $\begin{array}{c}\text { Energy } \\
\text { BSS/ } \\
\text { kcal mol }^{-1}\end{array}$ & $\begin{array}{c}\text { Energy } \\
\text { Yamaguchi/ }{\text { kcal } \text { mol }^{-1}}^{2}\end{array}$ \\
\hline 1.60 & 2.01 & 0.00 & 1.00 & 0.00 & 72.8 & 22.4 & 22.4 \\
\hline 1.80 & 2.01 & 0.00 & 1.00 & 0.00 & 43.6 & 1.9 & 1.9 \\
\hline 1.89 & 2.01 & 0.00 & 1.00 & 0.00 & 38.3 & 0.0 & 0.0 \\
\hline 2.00 & 2.01 & 0.00 & 1.00 & 0.00 & 35.8 & 1.1 & 1.1 \\
\hline 2.20 & 2.01 & 0.00 & 1.00 & 0.00 & 37.2 & 8.2 & 8.2 \\
\hline 2.40 & 2.01 & 0.00 & 1.00 & 0.00 & 42.0 & 17.8 & 17.8 \\
\hline 2.60 & 2.02 & 0.24 & 1.13 & 0.13 & 47.0 & 27.3 & 24.7 \\
\hline 2.80 & 2.03 & 0.51 & 1.34 & 0.34 & 48.1 & 34.0 & 29.1 \\
\hline 3.00 & 2.03 & 0.71 & 1.53 & 0.53 & 47.0 & 38.2 & 33.5 \\
\hline 3.20 & 2.03 & 0.83 & 1.70 & 0.70 & 46.2 & 40.9 & 37.1 \\
\hline 3.40 & 2.03 & 0.92 & 1.82 & 0.82 & 45.5 & 42.4 & 39.9 \\
\hline 3.60 & 2.03 & 0.96 & 1.91 & 0.91 & 45.0 & 43.3 & 41.7 \\
\hline
\end{tabular}




\section{Figures Part 1: Compound 1}
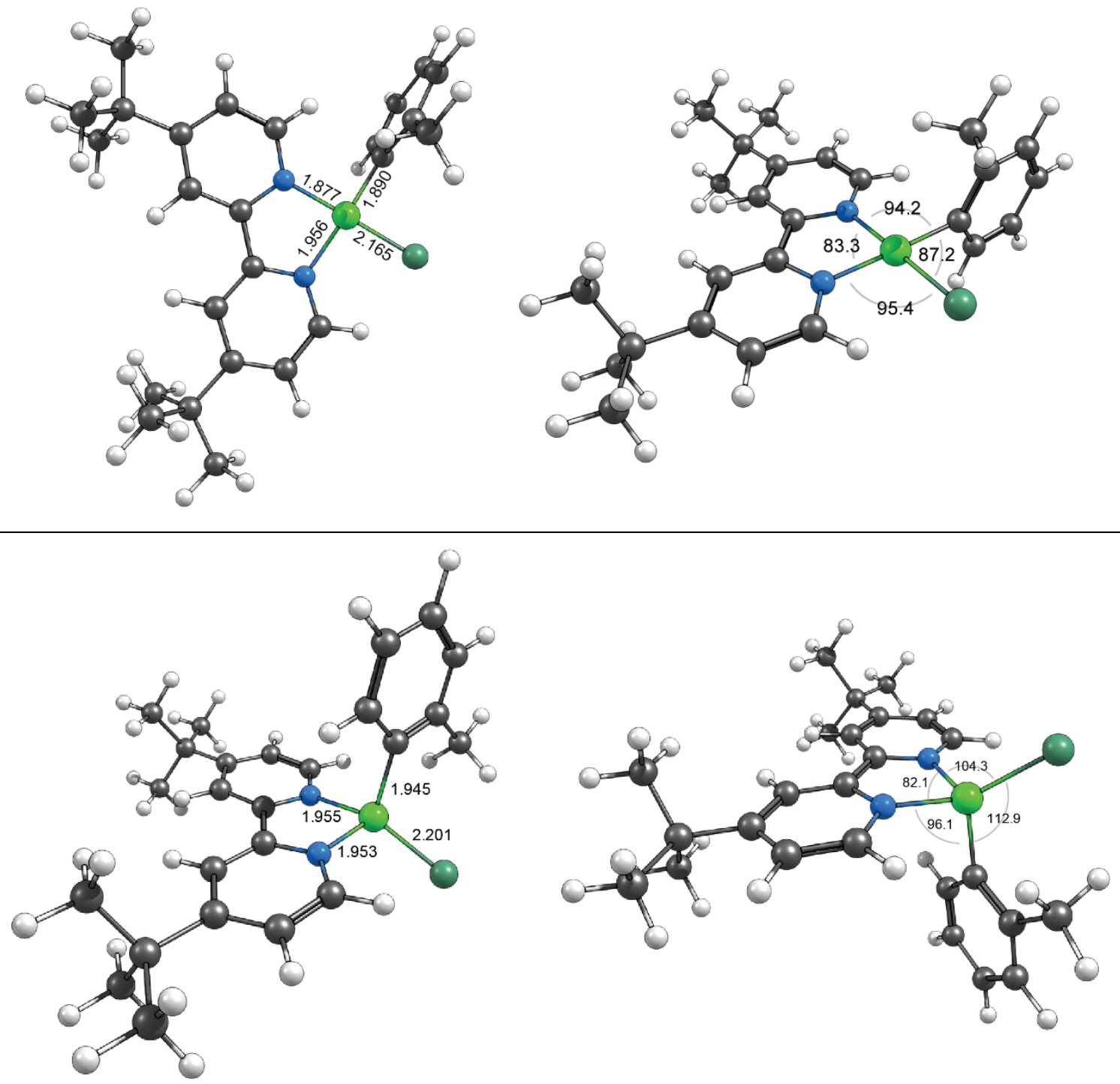

Figure S1A. Top: square planar equilibrium geometry of compound $1(S=0)$, and bottom: tetrahedral equilibrium geometry of $\mathbf{1}(\mathrm{S}=1)$ as calculated by DFT. Selected bond lengths and angles are shown. 


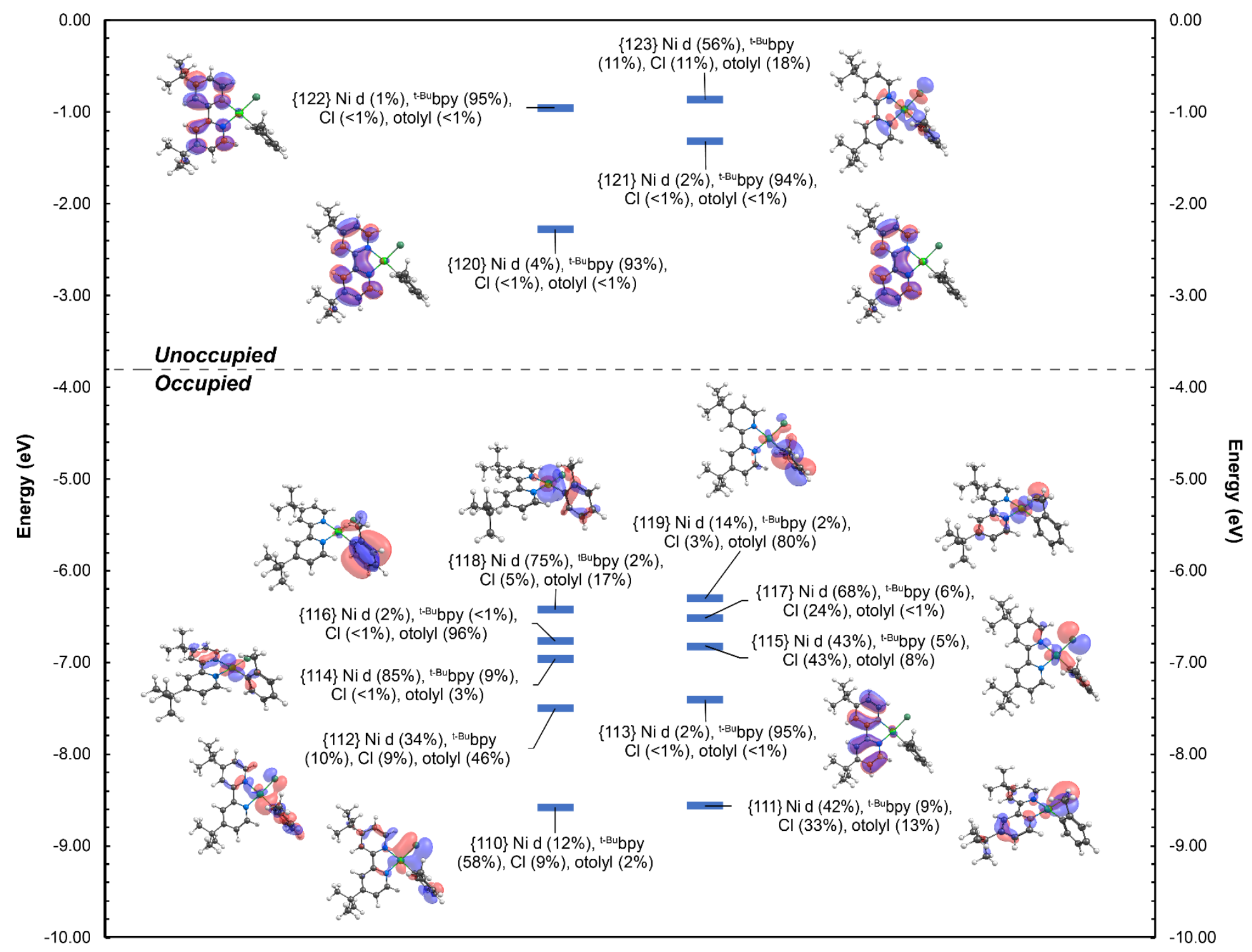

Figure S1B. Compound $1(\mathrm{~S}=0)$ - Single point DFT frontier molecular orbital diagram. Orbitals are offset for clarity. 


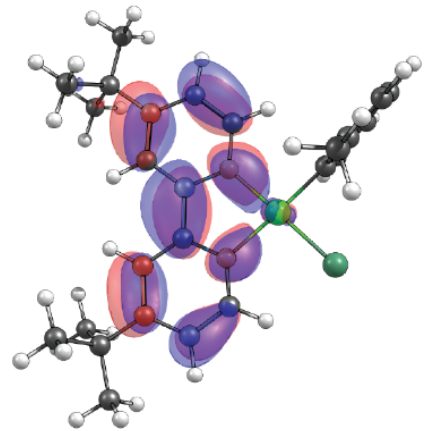

$\pi^{*}(1)$

Occ: 0.361

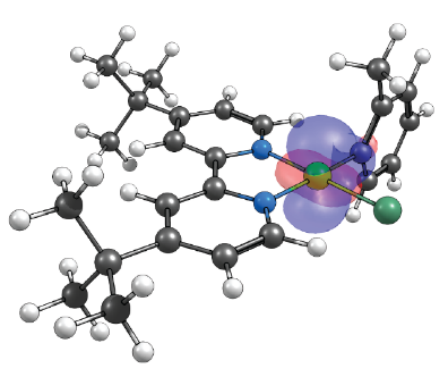

Occ: 1.688

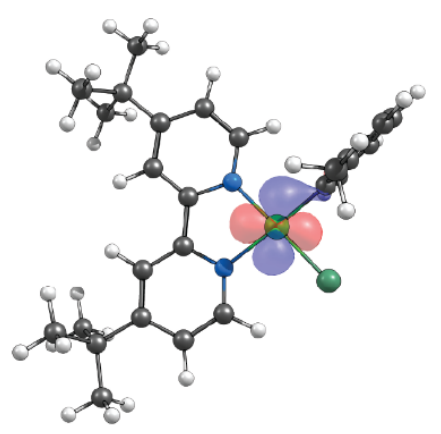

$d(x y)$
Occ: 1.769

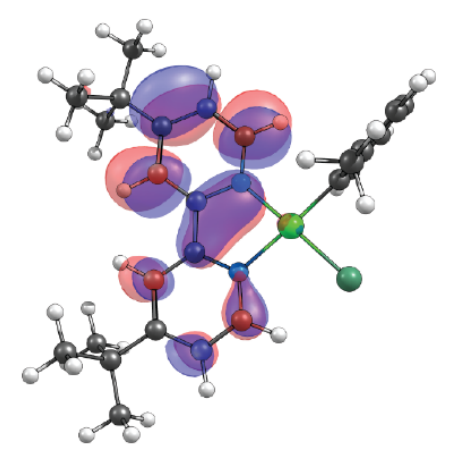

$\pi^{*}(2)$

Occ: 0.184

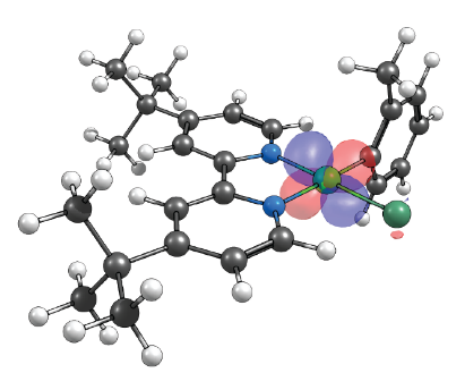

$$
\begin{gathered}
d(y z) \\
\text { Occ: } 1.686
\end{gathered}
$$

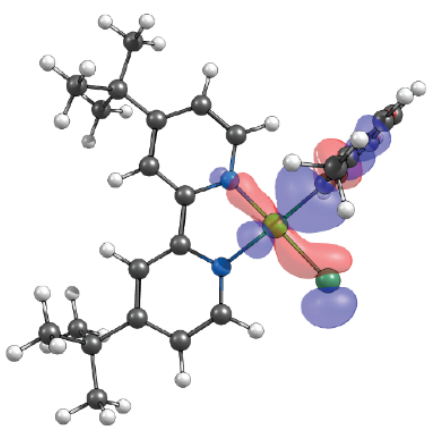

bonding $d\left(\mathbf{x}^{2}-\mathrm{y}^{2}\right) / \mathrm{C}\left(\mathbf{s p}^{2}\right)$

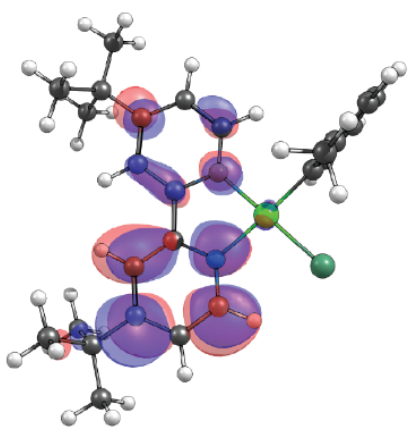

$\pi^{*}(3)$

Occ: 0.162

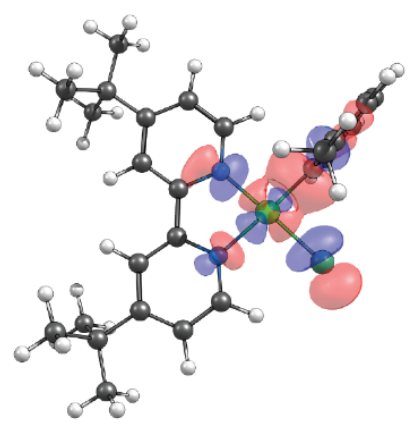

$d\left(x^{2}-y^{2}\right) / C\left(s p^{2}\right)^{*}$ Occ: 0.688

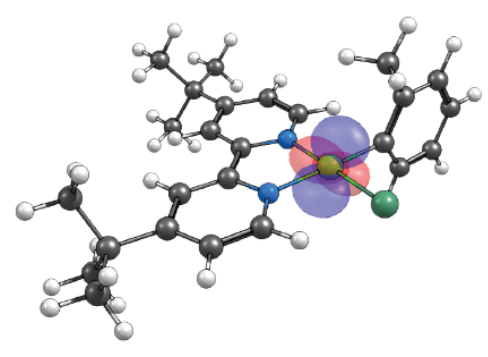

$d(x z)$

Figure S1C. Compound 1 (S=0) - CASSCF/QD-NEVPT2 9o, 10e Active Space. 


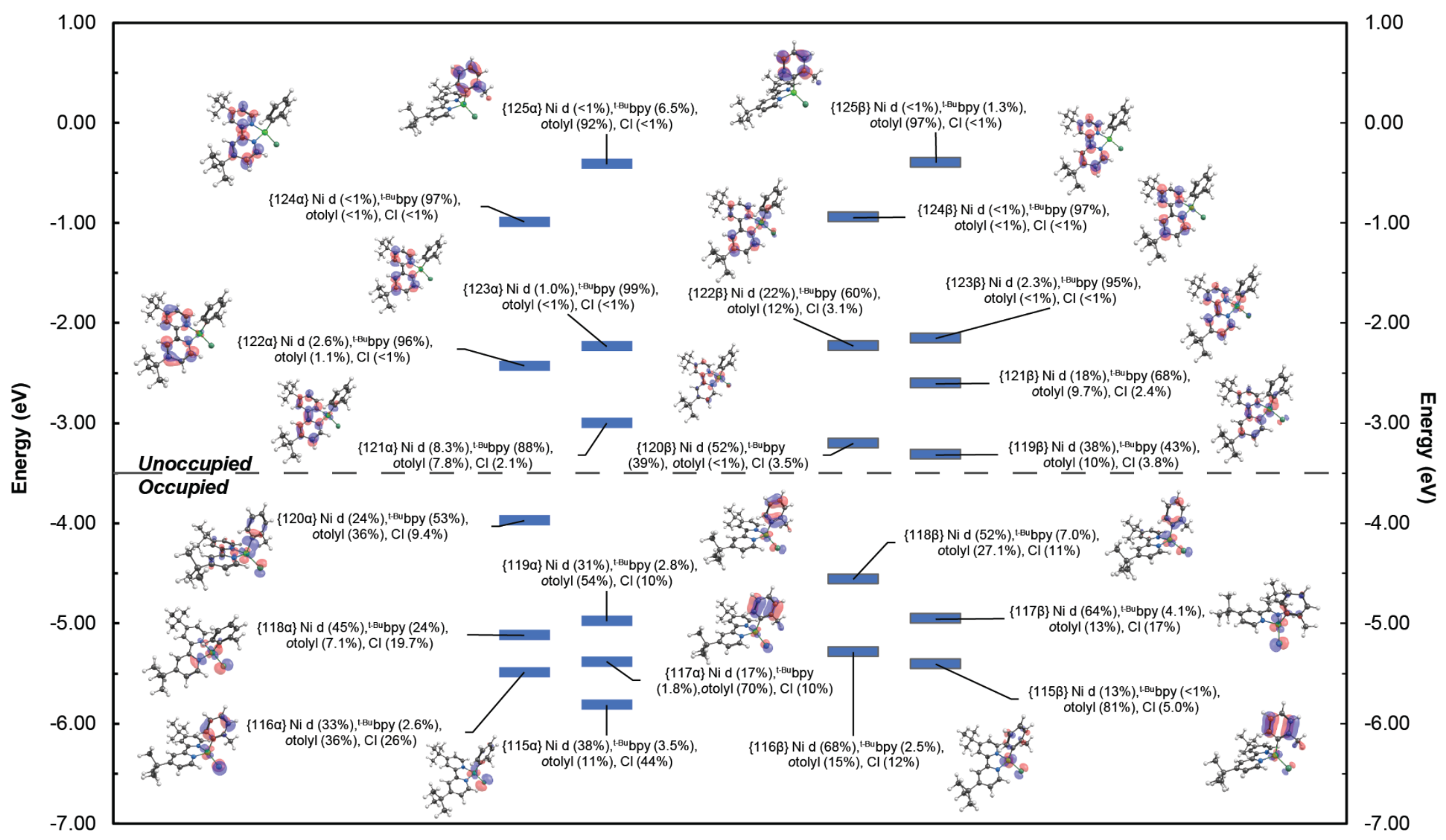

Figure S1D. Compound $1(S=1)$ - Single point DFT frontier molecular orbital diagram, with $\alpha$ orbitals on the left and $\beta$ orbitals on the right. $\alpha$ and $\beta$ orbitals are also offset for clarity. 

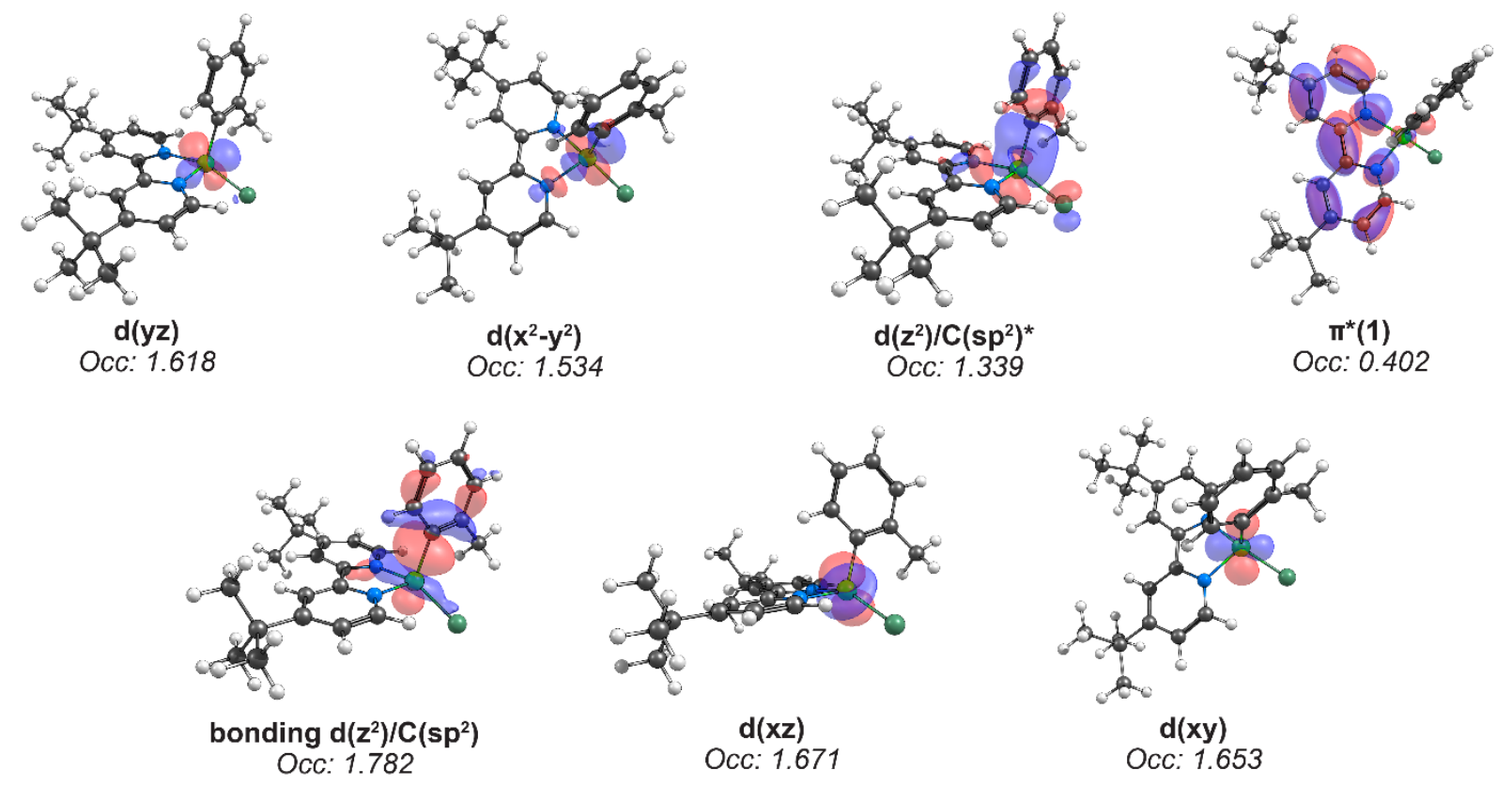

Figure S1E. Compound 1 (S=1) - CASSCF/QD-NEVPT2 7o, 10e Active Space.

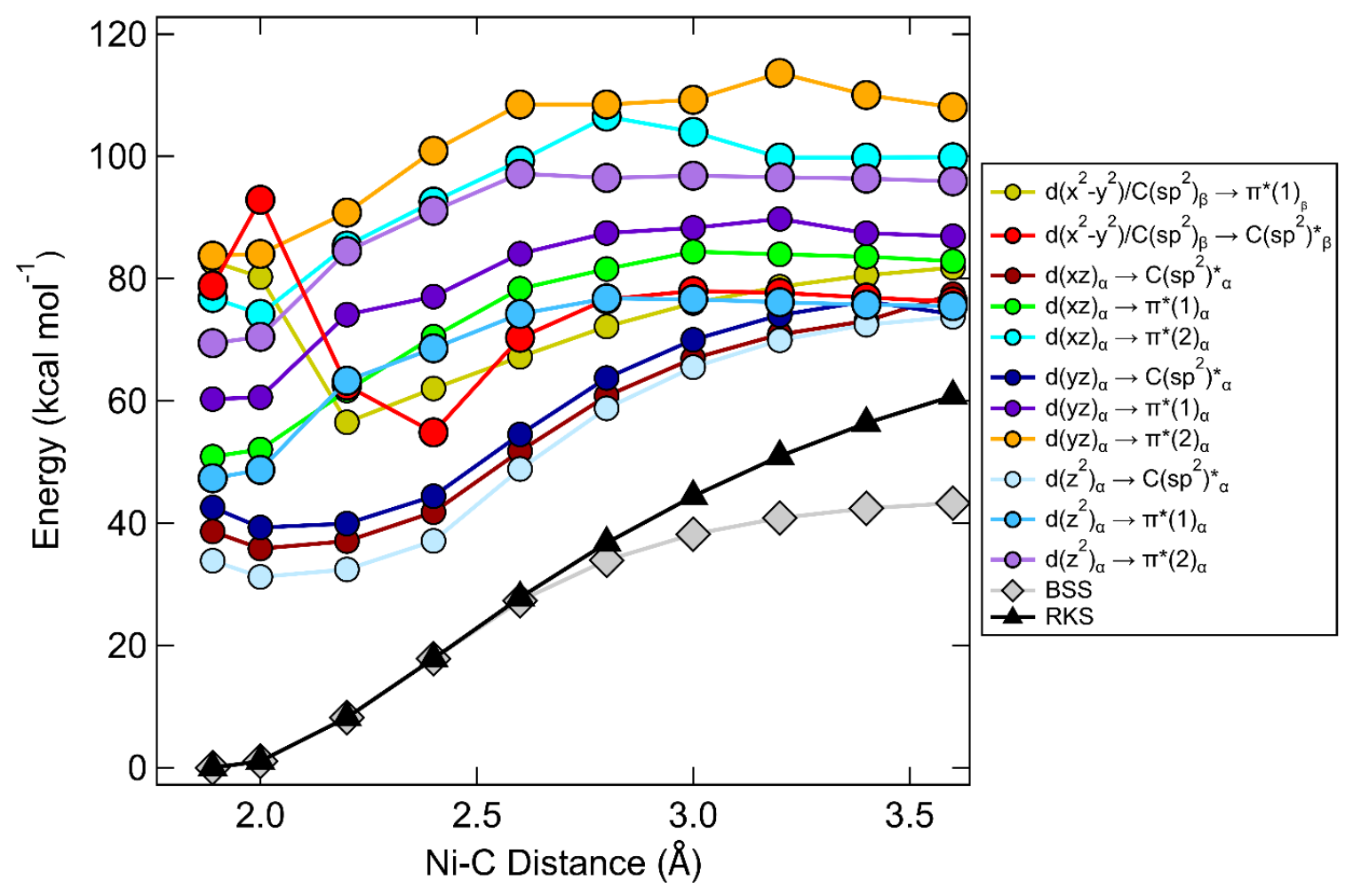

Figure S1F. Broken symmetry singlet TDDFT excitations along the $\mathrm{Ni}-\mathrm{C}$ coordinate of 1 show no repulsive excited states. Spin-contaminated BSS calculations gave a Ni-C BDE of 43.3 $\mathrm{kcal} \mathrm{mol}^{-1}$, while the Yamaguchi corrected $\mathrm{Ni}-\mathrm{C}$ BDE is only marginally different, at $41.7 \mathrm{kcal}$ $\mathrm{mol}^{-1}$. 


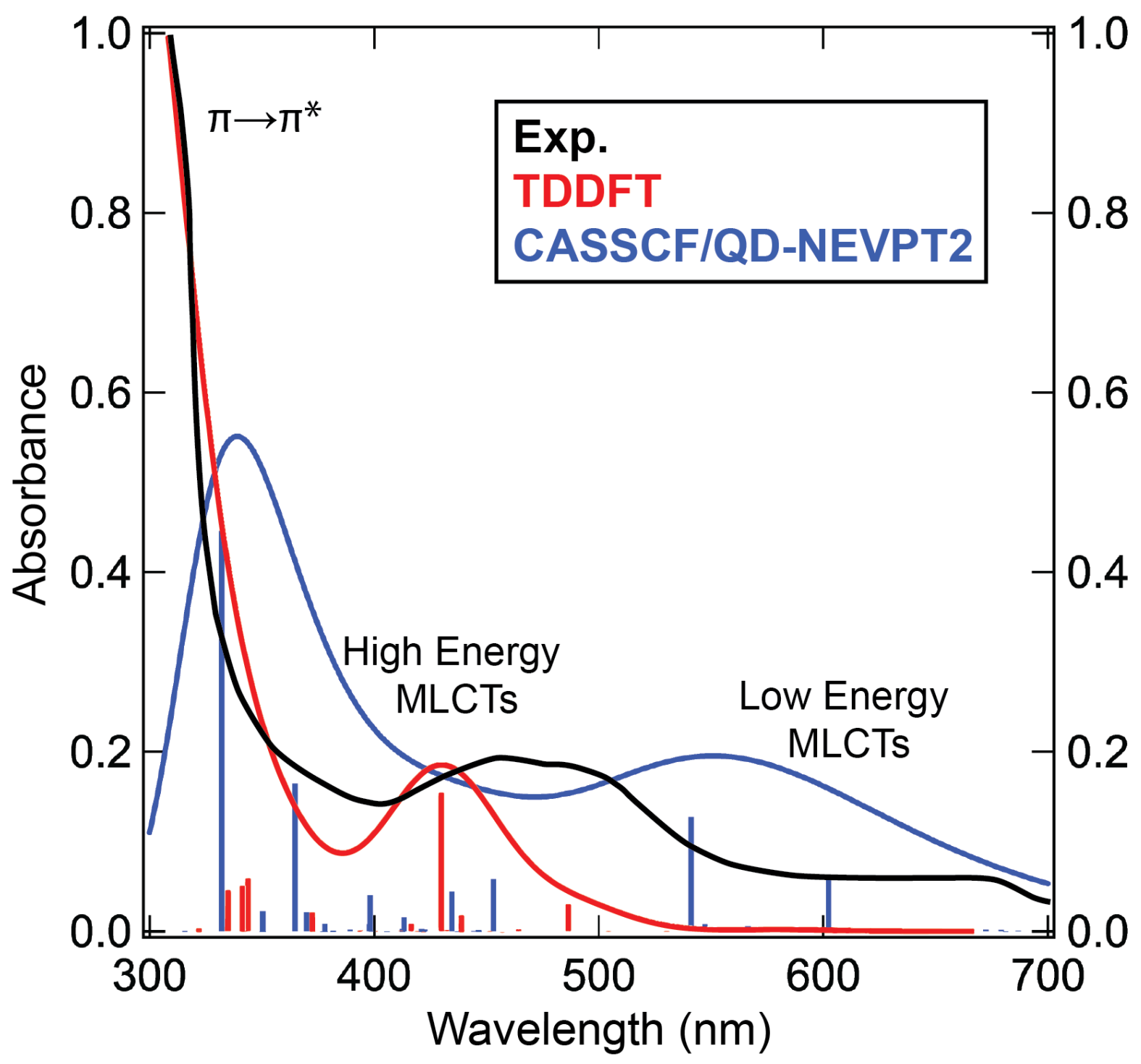

Figure S1G. Overlay of the experimental (THF) and calculated UV-vis spectrum of the equilibrium structure of $\mathbf{1}$. The TDDFT calculated spectrum (CPCM(THF)) in this research aligns well with that previously reported. ${ }^{3}$ The CASSCF/QD-NEVPT2 spectrum is spin-orbit coupling corrected with CPCM(THF). Note the relative oscillator strengths of the lower energy MLCT transitions are partially over-estimated in the CASSCF/QD-NEVPT2 calculated spectrum. This spectrum also does not display the intramolecular bpy-based $\pi \rightarrow \pi^{*}$ transitions, as the $\pi$ orbitals are not part of the active space. 


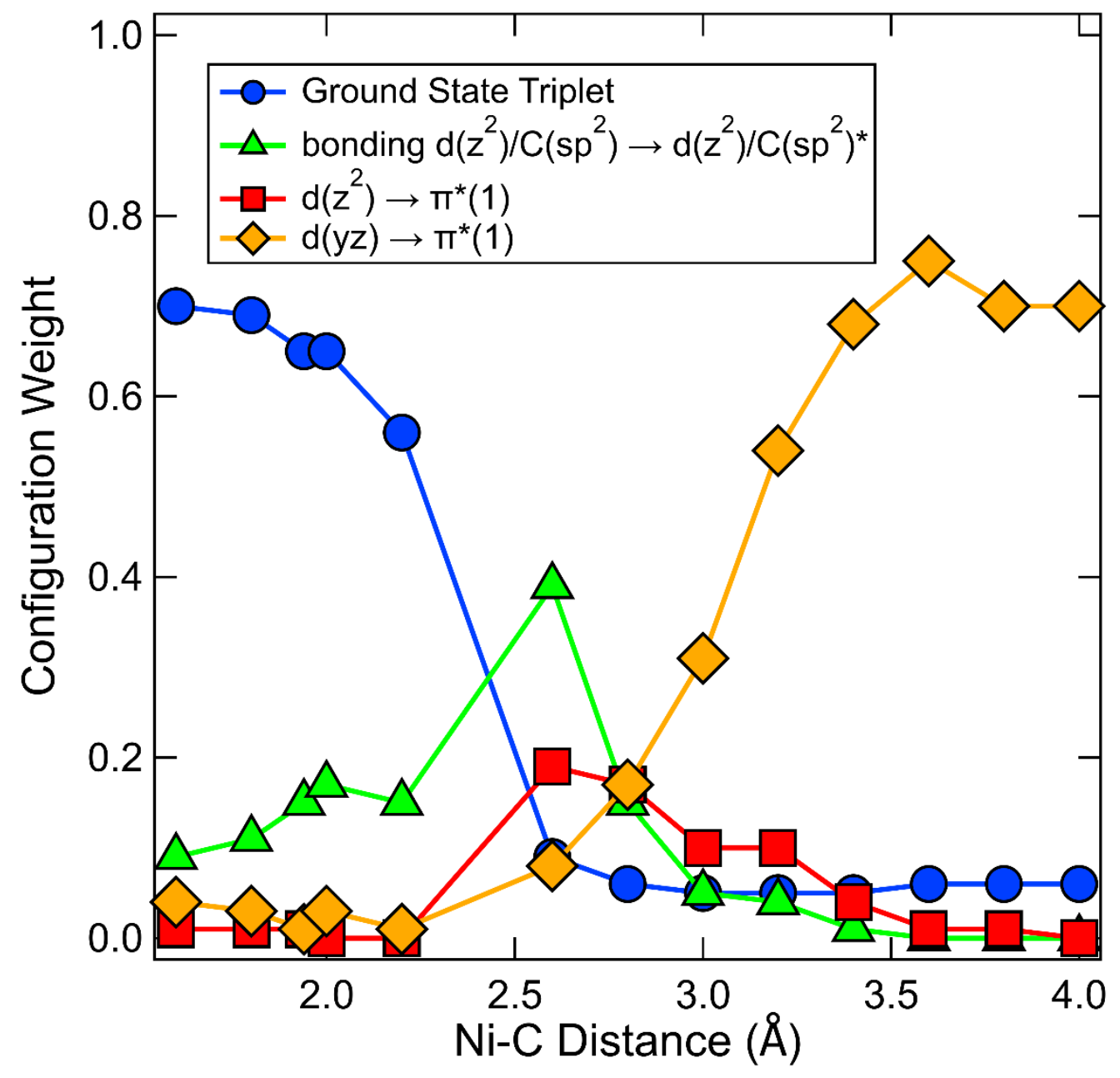

Figure S1H. Plot of the dominant configurations that contribute to the ground state CI vector along the optimized triplet $\mathrm{Ni}-\mathrm{C}$ bond elongation surface of $\mathbf{1}$. Note that for high spin $\mathbf{1}$, the ground state triplet is $\left[\mathrm{d}\left(\mathrm{x}^{2}-\mathrm{y}^{2}\right)\right]^{1}\left[\left(\mathrm{dz}^{2}\right) / \mathrm{C}\left(\mathrm{sp}^{2}\right)^{*}\right]^{1}$, making a $\mathrm{d}(\mathrm{yz}) \rightarrow \pi^{*}(1)$ transition yield an orbital configuration of $[\mathrm{d}(\mathrm{yz})]^{1}\left[\mathrm{~d}\left(\mathrm{x}^{2}-\mathrm{y}^{2}\right)\right]^{1}\left[\left(\mathrm{dz}^{2}\right) / \mathrm{C}\left(\mathrm{sp}^{2}\right)^{*}\right]^{1}\left[\pi^{*}(1)\right]^{1}$. 


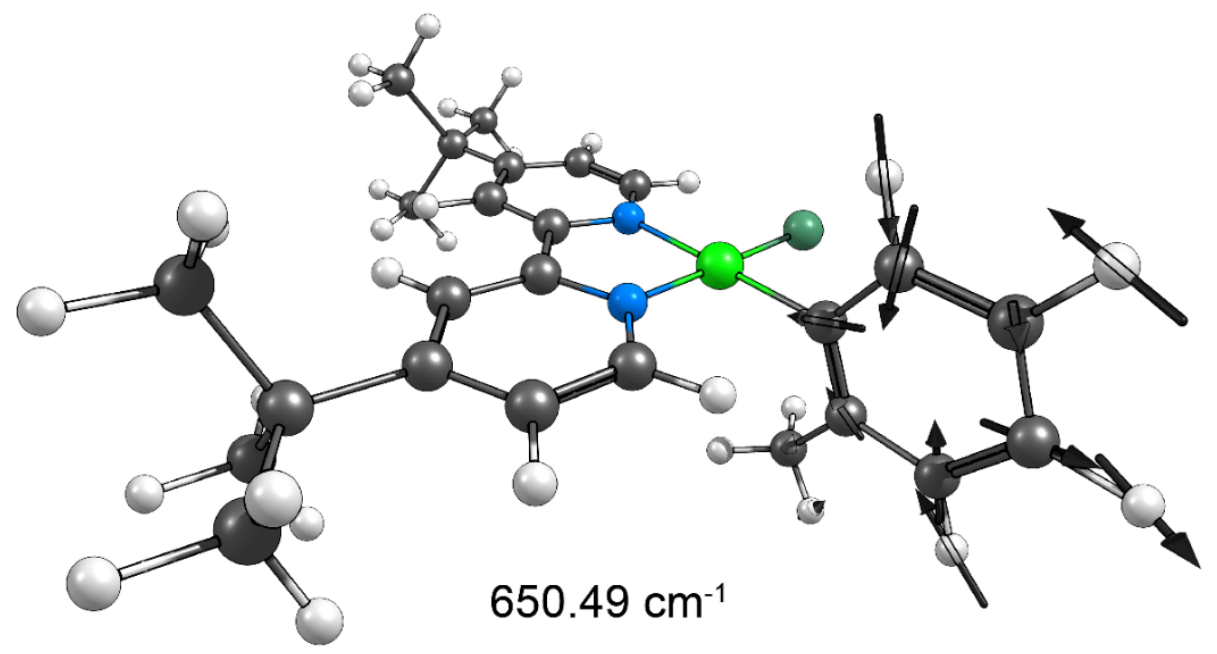

Figure S1I. Higher energy vibrational frequency in $\mathbf{1}$ that exhibits a $\mathrm{Ni}-\mathrm{C}$ bond stretching mode. 


\section{Tables Part 2: Compound 2}

Table S2A. Compound 2 - TDDFT lowest transition energies with CPCM(THF).

\begin{tabular}{|c|c|c|c|c|c|c|c|}
\hline State & Root & Multiplicity & $\Delta \mathrm{E} / \mathrm{nm}$ & $\Delta \mathrm{E} / \mathrm{kcal} \mathrm{mol}^{-1}$ & Transition & $f_{\text {osc }}$ & Contribution \\
\hline \multirow[t]{2}{*}{0} & 0 & 1 & --- & --- & --- & -- & --- \\
\hline & & & & & & --- & --- \\
\hline \multirow[t]{2}{*}{1} & 1 & 3 & 869.2 & 32.9 & $90 \mathrm{a} \rightarrow 94 \mathrm{a}$ & --- & 0.88196 \\
\hline & & & & & $84 a \rightarrow 94 a$ & --- & 0.03221 \\
\hline \multirow[t]{2}{*}{2} & 4 & 3 & 840.6 & 34.0 & $90 \mathrm{a} \rightarrow 91 \mathrm{a}$ & --- & 0.95884 \\
\hline & & & & & $90 \mathrm{a} \rightarrow 92 \mathrm{a}$ & --- & 0.01518 \\
\hline \multirow[t]{2}{*}{3} & 2 & 3 & 700.1 & 40.8 & $88 a \rightarrow 94 a$ & -- & 0.79230 \\
\hline & & & & & $87 a \rightarrow 94 a$ & --- & 0.11738 \\
\hline \multirow[t]{2}{*}{4} & 1 & 1 & 685.0 & 41.7 & $90 \mathrm{a} \rightarrow 91 \mathrm{a}$ & 0.0008022 & 0.97220 \\
\hline & & & & & --- & --- & --- \\
\hline \multirow[t]{2}{*}{5} & 13 & 3 & 654.9 & 43.7 & $87 a \rightarrow 94 a$ & --- & 0.76446 \\
\hline & & & & & $88 \mathrm{a} \rightarrow 94 \mathrm{a}$ & --- & 0.09933 \\
\hline \multirow[t]{2}{*}{6} & 3 & 3 & 647.7 & 44.1 & $89 a \rightarrow 91 a$ & --- & 0.76152 \\
\hline & & & & & $87 \mathrm{a} \rightarrow 91 \mathrm{a}$ & --- & 0.12192 \\
\hline \multirow[t]{2}{*}{7} & 8 & 3 & 621.1 & 46.0 & $88 a \rightarrow 91 a$ & --- & 0.84342 \\
\hline & & & & & $87 a \rightarrow 91 a$ & --- & 0.01896 \\
\hline \multirow[t]{2}{*}{8} & 6 & 3 & 597.5 & 47.8 & $87 a \rightarrow 91 a$ & --- & 0.80903 \\
\hline & & & & & $89 \mathrm{a} \rightarrow 91 \mathrm{a}$ & --- & 0.09728 \\
\hline \multirow[t]{2}{*}{9} & 7 & 3 & 561.6 & 50.9 & $89 a \rightarrow 94 a$ & --- & 0.27140 \\
\hline & & & & & $85 \mathrm{a} \rightarrow 94 \mathrm{a}$ & --- & 0.20986 \\
\hline \multirow[t]{2}{*}{10} & 5 & 3 & 515.1 & 55.5 & $88 a \rightarrow 91 a$ & --- & 0.43424 \\
\hline & & & & & $90 \mathrm{a} \rightarrow 94 \mathrm{a}$ & --- & 0.38209 \\
\hline \multirow[t]{2}{*}{11} & 2 & 1 & 499.1 & 57.3 & $88 a \rightarrow 91 a$ & 0.0049816 & 0.43424 \\
\hline & & & & & $90 \mathrm{a} \rightarrow 94 \mathrm{a}$ & --- & 0.38209 \\
\hline \multirow[t]{2}{*}{12} & 10 & 3 & 489.4 & 58.4 & $90 a \rightarrow 92 a$ & --- & 0.63866 \\
\hline & & & & & $85 a \rightarrow 91 a$ & --- & 0.28025 \\
\hline \multirow[t]{2}{*}{13} & 11 & 3 & 473.1 & 60.4 & $85 a \rightarrow 91 a$ & --- & 0.56985 \\
\hline & & & & & $90 \mathrm{a} \rightarrow 92 \mathrm{a}$ & --- & 0.32997 \\
\hline \multirow[t]{2}{*}{14} & 3 & 1 & 471.9 & 60.6 & $89 a \rightarrow 91 a$ & 0.0019717 & 0.60213 \\
\hline & & & & & $88 \mathrm{a} \rightarrow 94 \mathrm{a}$ & --- & 0.25483 \\
\hline \multirow[t]{2}{*}{15} & 12 & 3 & 456.8 & 62.6 & $90 a \rightarrow 93 a$ & --- & 0.97175 \\
\hline & & & & & --- & --- & --- \\
\hline \multirow[t]{2}{*}{16} & 9 & 1 & 437.6 & 65.3 & $90 a \rightarrow 92 a$ & 0.0004057 & 0.93755 \\
\hline & & & & & $85 \mathrm{a} \rightarrow 91 \mathrm{a}$ & --- & 0.02892 \\
\hline \multirow[t]{2}{*}{17} & 4 & 1 & 436.6 & 65.5 & $90 \mathrm{a} \rightarrow 94 \mathrm{a}$ & 0.0042122 & 0.44811 \\
\hline & & & & & $87 \mathrm{a} \rightarrow 91 \mathrm{a}$ & --- & 0.21201 \\
\hline \multirow[t]{2}{*}{18} & 11 & 1 & 428.9 & 66.7 & $86 a \rightarrow 91 a$ & 0.0009630 & 0.61475 \\
\hline & & & & & $90 \mathrm{a} \rightarrow 93 \mathrm{a}$ & --- & 0.33337 \\
\hline \multirow[t]{2}{*}{19} & 5 & 1 & 418.4 & 68.3 & $88 a \rightarrow 94 a$ & 0.0035092 & 0.61036 \\
\hline & & & & & $89 a \rightarrow 91 a$ & --- & 0.28106 \\
\hline \multirow[t]{2}{*}{20} & 8 & 1 & 414.8 & 68.9 & $87 a \rightarrow 91 a$ & 0.0473264 & 0.47012 \\
\hline & & & & & $88 \mathrm{a} \rightarrow 91 \mathrm{a}$ & --- & 0.21114 \\
\hline
\end{tabular}


Table S2B-1. Compound 2 - CASSCF/QD-NEVPT2 lowest transition energies in the gas phase. 5o, 8e Active Space. 10 triplet roots, 10 singlet roots. Orbitals (in order for CI vector notation below): $d\left(z^{2}\right), d(x z), d(y z), d(x y), d\left(x^{2}-y^{2}\right)$.

\begin{tabular}{|c|c|c|c|c|c|c|c|}
\hline State & Root & Multiplicity & $\Delta \mathbf{E} / \mathbf{n m}$ & $\Delta \mathrm{E} / \mathrm{kcal} \mathrm{mol}^{-1}$ & CI Vector & $f_{\text {osc }}$ & Contribution \\
\hline \multirow[t]{2}{*}{0} & 0 & 1 & --- & --- & 22220 & --- & 0.9515 \\
\hline & & & & & 22022 & --- & 0.0127 \\
\hline \multirow[t]{2}{*}{1} & 0 & 3 & 1175.9 & 24.3 & 12221 & --- & 0.9996 \\
\hline & & & & & --- & --- & --- \\
\hline \multirow[t]{2}{*}{2} & 1 & 3 & 963.0 & 29.7 & 22121 & --- & 0.8786 \\
\hline & & & & & 21221 & --- & 0.0926 \\
\hline \multirow[t]{2}{*}{3} & 2 & 3 & 891.6 & 32.1 & 21221 & --- & 0.8834 \\
\hline & & & & & 22121 & --- & 0.0919 \\
\hline \multirow[t]{2}{*}{4} & 3 & 3 & 627.0 & 45.6 & 22211 & --- & 0.9394 \\
\hline & & & & & 21122 & --- & 0.0578 \\
\hline \multirow[t]{2}{*}{5} & 1 & 1 & 420.7 & 68.0 & 12221 & 0.0000621 & 0.9806 \\
\hline & & & & & 20222 & & 0.0088 \\
\hline \multirow[t]{2}{*}{6} & 2 & 1 & 407.6 & 70.1 & 22121 & 0.0000131 & 0.9097 \\
\hline & & & & & 21221 & & 0.0496 \\
\hline \multirow[t]{2}{*}{7} & 3 & 1 & 391.7 & 73.0 & 21221 & 0.0001101 & 0.9245 \\
\hline & & & & & 22121 & & 0.0488 \\
\hline \multirow[t]{2}{*}{8} & 4 & 1 & 385.2 & 74.2 & 22211 & 0.0000915 & 0.9826 \\
\hline & & & & & 22121 & & 0.0155 \\
\hline \multirow[t]{2}{*}{9} & 4 & 3 & 293.2 & 97.5 & 12212 & --- & 0.9890 \\
\hline & & & & & 22112 & --- & 0.0055 \\
\hline \multirow[t]{2}{*}{10} & 5 & 3 & 288.5 & 99.1 & 22112 & --- & 0.7613 \\
\hline & & & & & 21212 & --- & 0.0649 \\
\hline
\end{tabular}


Table S2B-2. Compound 2 - CASSCF/QD-NEVPT2 lowest transition energies with CPCM(THF). 5o, 8e Active Space - 10 triplet roots, 10 singlet roots. Orbitals (in order for CI vector notation below): $\mathrm{d}\left(\mathrm{z}^{2}\right), \mathrm{d}(\mathrm{xz}), \mathrm{d}(\mathrm{yz}), \mathrm{d}(\mathrm{xy}), \mathrm{d}\left(\mathrm{x}^{2}-\mathrm{y}^{2}\right)$.

\begin{tabular}{|c|c|c|c|c|c|c|c|}
\hline State & Root & Multiplicity & $\Delta \mathrm{E} / \mathrm{nm}$ & $\Delta \mathrm{E} / \mathrm{kcal} \mathrm{mol}^{-1}$ & CI Vector & $f_{\text {osc }}$ & Contribution \\
\hline \multirow[t]{2}{*}{0} & 0 & 1 & --- & --- & 22220 & --- & 0.9510 \\
\hline & & & & & 02222 & --- & 0.0211 \\
\hline \multirow[t]{2}{*}{1} & 0 & 3 & 1237.6 & 23.1 & 12221 & --- & 0.9992 \\
\hline & & & & & --- & --- & --- \\
\hline \multirow[t]{2}{*}{2} & 1 & 3 & 1011.0 & 28.3 & 22121 & --- & 0.9053 \\
\hline & & & & & 21221 & --- & 0.0672 \\
\hline \multirow[t]{2}{*}{3} & 2 & 3 & 941.0 & 30.4 & 21221 & --- & 0.9075 \\
\hline & & & & & 22121 & --- & 0.0668 \\
\hline \multirow[t]{2}{*}{4} & 3 & 3 & 635.5 & 45.0 & 22211 & --- & 0.9379 \\
\hline & & & & & 21122 & --- & 0.0611 \\
\hline \multirow[t]{2}{*}{5} & 1 & 1 & 426.9 & 67.0 & 12221 & 0.0000485 & 0.9796 \\
\hline & & & & & 20222 & --- & 0.0090 \\
\hline \multirow[t]{2}{*}{6} & 2 & 1 & 414.6 & 69.0 & 22121 & 0.0000040 & 0.9339 \\
\hline & & & & & 21221 & --- & 0.0351 \\
\hline \multirow[t]{2}{*}{7} & 3 & 1 & 399.8 & 71.5 & 21221 & 0.0001066 & 0.9353 \\
\hline & & & & & 22121 & --- & 0.0359 \\
\hline \multirow[t]{2}{*}{8} & 4 & 1 & 387.0 & 73.9 & 22211 & 0.0000935 & 0.9944 \\
\hline & & & & & 22121 & --- & 0.0048 \\
\hline \multirow[t]{2}{*}{9} & 4 & 3 & 297.1 & 96.2 & 12212 & --- & 0.9759 \\
\hline & & & & & 21212 & --- & 0.0145 \\
\hline \multirow[t]{2}{*}{10} & 5 & 3 & 293.3 & 97.5 & 22112 & --- & 0.7716 \\
\hline & & & & & 11222 & --- & 0.1477 \\
\hline
\end{tabular}


Table S2C-1. Compound 2 - CASSCF/QD-NEVPT2 lowest transition energies in the gas phase. 9o, 10e Active Space -25 triplet roots, 15 singlet roots. Orbitals (in order for CI vector notation below): bonding $\mathrm{d}\left(\mathrm{x}^{2}-\mathrm{y}^{2}\right) / \mathrm{C}\left(\mathrm{sp}^{2}\right), \mathrm{d}(\mathrm{xy}), \mathrm{d}(\mathrm{xz}), \mathrm{d}(\mathrm{yz}), \mathrm{d}\left(\mathrm{z}^{2}\right), \mathrm{d}\left(\mathrm{x}^{2}-\mathrm{y}^{2}\right) / \mathrm{C}\left(\mathrm{sp}^{2}\right)^{*}, \pi^{*}(1), \pi^{*}(2), \pi^{*}(3)$.

\begin{tabular}{|c|c|c|c|c|c|c|c|}
\hline State & Root & Multiplicity & $\Delta \mathrm{E} / \mathrm{nm}$ & $\Delta \mathrm{E} / \mathrm{kcal} \mathrm{mol}^{-1}$ & CI Vector & $f_{\text {osc }}$ & Contribution \\
\hline \multirow[t]{3}{*}{0} & 0 & 1 & --- & --- & 222220000 & --- & 0.5464 \\
\hline & & & & & 222120100 & --- & 0.1039 \\
\hline & & & & & 122221000 & --- & 0.0858 \\
\hline \multirow[t]{2}{*}{1} & 0 & 3 & 970.1 & 29.5 & 222211000 & --- & 0.4539 \\
\hline & & & & & 122212000 & --- & 0.1696 \\
\hline \multirow[t]{2}{*}{2} & 1 & 3 & 804.6 & 35.5 & 222121000 & $\begin{array}{c}-- \\
\end{array}$ & 0.6017 \\
\hline & & & & & 212221000 & --- & 0.1400 \\
\hline \multirow[t]{2}{*}{3} & 2 & 3 & 758.2 & 37.7 & 212221000 & --- & 0.5703 \\
\hline & & & & & 222121000 & --- & 0.1536 \\
\hline \multirow[t]{2}{*}{4} & 3 & 3 & 737.1 & 38.8 & 222210100 & --- & 0.5396 \\
\hline & & & & & 122220100 & --- & 0.2880 \\
\hline \multirow[t]{2}{*}{5} & 1 & 1 & 729.4 & 39.2 & 222210100 & 0.0019683 & 0.5703 \\
\hline & & & & & 122220100 & --- & 0.2712 \\
\hline \multirow[t]{2}{*}{6} & 4 & 3 & 642.1 & 44.5 & 222120100 & --- & 0.8142 \\
\hline & & & & & 122112100 & --- & 0.0417 \\
\hline \multirow[t]{2}{*}{7} & 2 & 1 & 624.3 & 45.8 & 222120100 & 0.0905865 & 0.6714 \\
\hline & & & & & 212220100 & --- & 0.0655 \\
\hline \multirow[t]{2}{*}{8} & 5 & 3 & 592.5 & 48.3 & 221221000 & --- & 0.6680 \\
\hline & & & & & 212122000 & --- & 0.0822 \\
\hline \multirow[t]{2}{*}{9} & 6 & 3 & 571.6 & 50.0 & 212220100 & --- & 0.7675 \\
\hline & & & & & 212202100 & --- & 0.0438 \\
\hline \multirow[t]{2}{*}{10} & 3 & 1 & 569.9 & 50.2 & 212220100 & 0.1206524 & 0.6956 \\
\hline & & & & & 112212100 & --- & 0.0370 \\
\hline \multirow[t]{2}{*}{11} & 4 & 1 & 444.4 & 64.3 & 221220100 & 0.0003508 & 0.7306 \\
\hline & & & & & 212121100 & --- & 0.0444 \\
\hline \multirow[t]{2}{*}{12} & 7 & 3 & 433.9 & 65.9 & 222210010 & --- & 0.4812 \\
\hline & & & & & 122220010 & --- & 0.2506 \\
\hline \multirow[t]{2}{*}{13} & 5 & 1 & 432.7 & 66.1 & 222210010 & 0.0010716 & 0.4782 \\
\hline & & & & & 122220010 & --- & 0.2503 \\
\hline \multirow[t]{2}{*}{14} & 8 & 3 & 429.9 & 66.5 & 221220100 & --- & 0.7217 \\
\hline & & & & & 212121100 & --- & 0.0699 \\
\hline \multirow[t]{2}{*}{15} & 6 & 1 & 410.4 & 69.7 & 222120010 & 0.0274877 & 0.3593 \\
\hline & & & & & 222211000 & --- & 0.1504 \\
\hline \multirow[t]{2}{*}{16} & 7 & 1 & 404.5 & 70.7 & 222120001 & 0.0141815 & 0.2505 \\
\hline & & & & & 222211000 & --- & 0.1880 \\
\hline \multirow[t]{2}{*}{17} & 9 & 3 & 400.6 & 71.4 & 222120010 & --- & 0.7918 \\
\hline & & & & & 122112010 & --- & 0.0430 \\
\hline \multirow[t]{2}{*}{18} & 8 & 1 & 399.8 & 71.5 & 222121000 & 0.0076735 & 0.4769 \\
\hline & & & & & 222120010 & --- & 0.0742 \\
\hline \multirow[t]{2}{*}{19} & 9 & 1 & 396.4 & 72.1 & 222210001 & 0.0025346 & 0.3590 \\
\hline & & & & & 122220001 & --- & 0.1937 \\
\hline \multirow[t]{2}{*}{20} & 10 & 3 & 389.0 & 73.5 & 222210001 & --- & 0.5095 \\
\hline & & & & & 122220001 & --- & 0.2709 \\
\hline
\end{tabular}


Table S2C-2. Compound 2 - CASSCF/QD-NEVPT2 lowest transition energies with CPCM(THF). 9o, 10e Active Space - 25 triplet roots, 15 singlet roots. Orbitals (in order for CI vector notation below): bonding $\mathrm{d}\left(\mathrm{x}^{2}-\mathrm{y}^{2}\right) / \mathrm{C}\left(\mathrm{sp}^{2}\right), \mathrm{d}(\mathrm{xy}), \mathrm{d}(\mathrm{xz}), \mathrm{d}(\mathrm{yz}), \mathrm{d}\left(\mathrm{z}^{2}\right), \mathrm{d}\left(\mathrm{x}^{2}-\mathrm{y}^{2}\right) / \mathrm{C}\left(\mathrm{sp}^{2}\right)^{*}, \pi^{*}(1)$, $\pi^{*}(2), \pi^{*}(3)$.

\begin{tabular}{|c|c|c|c|c|c|c|c|}
\hline State & Root & Multiplicity & $\Delta \mathrm{E} / \mathrm{nm}$ & $\Delta \mathrm{E} / \mathrm{kcal} \mathrm{mol}^{-1}$ & CI Vector & $f_{\text {osc }}$ & Contribution \\
\hline \multirow[t]{3}{*}{0} & 0 & 1 & --- & --- & 222220000 & --- & 0.5730 \\
\hline & & & & & 222120100 & --- & 0.1468 \\
\hline & & & & & 122221000 & --- & 0.0958 \\
\hline \multirow[t]{2}{*}{1} & 0 & 3 & 881.1 & 32.4 & 222211000 & --- & 0.7022 \\
\hline & & & & & 122212000 & --- & 0.1274 \\
\hline \multirow[t]{2}{*}{2} & 1 & 3 & 794.6 & 36.0 & 222210100 & --- & 0.7593 \\
\hline & & & & & 022212100 & --- & 0.0692 \\
\hline \multirow[t]{2}{*}{3} & 1 & 1 & 768.5 & 37.2 & 222210100 & 0.0013739 & 0.7523 \\
\hline & & & & & 022212100 & --- & 0.0686 \\
\hline \multirow[t]{2}{*}{4} & 2 & 3 & 747.1 & 38.3 & 221221000 & --- & 0.4723 \\
\hline & & & & & 222121000 & --- & 0.2884 \\
\hline \multirow[t]{2}{*}{5} & 3 & 3 & 698.1 & 41.0 & 222121000 & --- & 0.3430 \\
\hline & & & & & 221221000 & --- & 0.2101 \\
\hline \multirow[t]{2}{*}{6} & 4 & 3 & 681.7 & 41.9 & 222120100 & --- & 0.5581 \\
\hline & & & & & 222121000 & --- & 0.1158 \\
\hline \multirow[t]{2}{*}{7} & 2 & 1 & 645.2 & 44.3 & 221220100 & 0.0410929 & 0.4915 \\
\hline & & & & & 222120100 & --- & 0.2839 \\
\hline \multirow[t]{2}{*}{8} & 5 & 3 & 606.9 & 47.1 & 221220100 & --- & 0.7427 \\
\hline & & & & & 222120100 & --- & 0.0615 \\
\hline \multirow[t]{2}{*}{9} & 3 & 1 & 577.4 & 49.5 & 221220100 & 0.1644325 & 0.3046 \\
\hline & & & & & 222120100 & --- & 0.2834 \\
\hline \multirow[t]{2}{*}{10} & 6 & 3 & 570.0 & 50.2 & 212221000 & --- & 0.7017 \\
\hline & & & & & 221122000 & --- & 0.0669 \\
\hline \multirow[t]{2}{*}{11} & 4 & 1 & 461.1 & 62.0 & 212220100 & 0.0001703 & 0.7321 \\
\hline & & & & & 221121100 & --- & 0.0719 \\
\hline \multirow[t]{2}{*}{12} & 7 & 3 & 451.1 & 63.4 & 222210010 & --- & 0.3902 \\
\hline & & & & & 212220100 & --- & 0.3515 \\
\hline \multirow[t]{2}{*}{13} & 8 & 3 & 449.1 & 63.7 & 212220100 & --- & 0.4083 \\
\hline & & & & & 222210010 & --- & 0.3346 \\
\hline \multirow[t]{2}{*}{14} & 5 & 1 & 448.7 & 63.7 & 222210010 & 0.0030332 & 0.6533 \\
\hline & & & & & 222120010 & --- & 0.0760 \\
\hline \multirow[t]{2}{*}{15} & 6 & 1 & 439.7 & 65.0 & 222120010 & 0.1016451 & 0.4437 \\
\hline & & & & & 221220010 & --- & 0.1562 \\
\hline \multirow[t]{2}{*}{16} & 9 & 3 & 416.2 & 68.7 & 222120010 & --- & 0.7280 \\
\hline & & & & & 22122010 & --- & 0.0705 \\
\hline \multirow[t]{2}{*}{17} & 7 & 1 & 413.6 & 69.1 & 222210001 & 0.0097205 & 0.5953 \\
\hline & & & & & 222211000 & --- & 0.1138 \\
\hline \multirow[t]{2}{*}{18} & 10 & 3 & 402.8 & 71.0 & 222210001 & --- & 0.7274 \\
\hline & & & & & 022212001 & --- & 0.0661 \\
\hline \multirow[t]{2}{*}{19} & 8 & 1 & 397.2 & 72.0 & 222121000 & 0.0315108 & 0.2618 \\
\hline & & & & & 222120001 & --- & 0.1434 \\
\hline \multirow[t]{2}{*}{20} & 9 & 1 & 392.8 & 72.8 & 222121000 & 0.0000160 & 0.2800 \\
\hline & & & & & 212221000 & --- & 0.2335 \\
\hline
\end{tabular}


Table S2C-3. Compound 2 - CASSCF/QD-NEVPT2 lowest transition energies in gas phase 9o, 10e Active Space -25 triplet roots, 25 singlet roots. Orbitals (in order for CI vector notation below): $\mathrm{d}\left(\mathrm{z}^{2}\right), \mathrm{d}(\mathrm{xz}), \mathrm{d}(\mathrm{xy}), \mathrm{d}(\mathrm{yz})$, bonding $\mathrm{d}\left(\mathrm{x}^{2}-\mathrm{y}^{2}\right) / \mathrm{C}\left(\mathrm{sp}^{2}\right), \mathrm{d}\left(\mathrm{x}^{2}-\mathrm{y}^{2}\right) / \mathrm{C}\left(\mathrm{sp}^{2}\right)^{*}, \pi^{*}(1), \pi^{*}(2), \pi^{*}(3)$.

\begin{tabular}{|c|c|c|c|c|c|c|c|}
\hline State & Root & Multiplicity & $\Delta \mathrm{E} / \mathrm{nm}$ & $\Delta \mathrm{E} / \mathrm{kcal} \mathrm{mol}^{-1}$ & CI Vector & $f_{\text {osc }}$ & Contribution \\
\hline \multirow[t]{3}{*}{0} & 0 & 1 & -- & -- & 222220000 & -- & 0.5529 \\
\hline & & & & & 222211000 & --- & 0.1358 \\
\hline & & & & & 222120100 & --- & 0.0884 \\
\hline \multirow[t]{2}{*}{1} & 0 & 3 & 1069.5 & 26.7 & 122221000 & --- & 0.4330 \\
\hline & & & & & 222211000 & --- & 0.2996 \\
\hline \multirow[t]{2}{*}{2} & 1 & 3 & 814.6 & 35.1 & 212221000 & $\begin{array}{ll}-- \\
--\end{array}$ & 0.4162 \\
\hline & & & & & 222121000 & --- & 0.3044 \\
\hline \multirow[t]{2}{*}{3} & 2 & 3 & 776.5 & 36.8 & 222121000 & --- & 0.4340 \\
\hline & & & & & 212221000 & --- & 0.2879 \\
\hline \multirow[t]{2}{*}{4} & 3 & 3 & 739.2 & 38.7 & 122220100 & --- & 0.4187 \\
\hline & & & & & 222210100 & --- & 0.3986 \\
\hline \multirow[t]{2}{*}{5} & 1 & 1 & 731.2 & 39.1 & 122220100 & 0.0032771 & 0.4253 \\
\hline & & & & & 222210100 & --- & 0.3978 \\
\hline \multirow[t]{2}{*}{6} & 4 & 3 & 644.6 & 44.4 & 222120100 & -- & 0.8188 \\
\hline & & & & & 122112100 & --- & 0.0443 \\
\hline \multirow[t]{2}{*}{7} & 2 & 1 & 634.3 & 45.1 & 222120100 & 0.1012976 & 0.7104 \\
\hline & & & & & 222211000 & --- & 0.0384 \\
\hline \multirow[t]{2}{*}{8} & 5 & 3 & 568.6 & 50.3 & 212220100 & --- & 0.4565 \\
\hline & & & & & 221221000 & --- & 0.2881 \\
\hline \multirow[t]{2}{*}{9} & 3 & 1 & 565.3 & 50.6 & 212220100 & 0.0896286 & 0.7351 \\
\hline & & & & & 212202100 & --- & 0.0675 \\
\hline \multirow[t]{2}{*}{10} & 6 & 3 & 558.8 & 51.2 & 221221000 & -- & 0.3895 \\
\hline & & & & & 212220100 & --- & 0.3283 \\
\hline \multirow[t]{2}{*}{11} & 7 & 3 & 471.8 & 60.6 & 221220100 & --- & 0.6713 \\
\hline & & & & & 212121100 & --- & 0.0636 \\
\hline \multirow[t]{2}{*}{12} & 8 & 3 & 467.7 & 61.1 & 122220010 & --- & 0.3885 \\
\hline & & & & & 222210010 & --- & 0.3303 \\
\hline \multirow[t]{2}{*}{13} & 4 & 1 & 448.0 & 63.8 & 221220100 & 0.0026610 & 0.7014 \\
\hline & & & & & 221202100 & --- & 0.0521 \\
\hline \multirow[t]{2}{*}{14} & 5 & 1 & 436.5 & 65.5 & 122220010 & 0.0021410 & 0.3993 \\
\hline & & & & & 222210010 & --- & 0.3394 \\
\hline \multirow[t]{2}{*}{15} & 6 & 1 & 421.5 & 67.8 & 222120010 & 0.0471175 & 0.2936 \\
\hline & & & & & 122221000 & --- & 0.1924 \\
\hline \multirow[t]{2}{*}{16} & 9 & 3 & 406.0 & 70.4 & 222120010 & -- & 0.7977 \\
\hline & & & & & 122112010 & --- & 0.0460 \\
\hline \multirow[t]{2}{*}{17} & 7 & 1 & 398.6 & 71.7 & 222121000 & 0.0012705 & 0.6168 \\
\hline & & & & & 122122000 & --- & 0.0491 \\
\hline \multirow[t]{2}{*}{18} & 8 & 1 & 397.8 & 71.9 & 222120010 & 0.0337901 & 0.2880 \\
\hline & & & & & 122221000 & --- & 0.1805 \\
\hline \multirow[t]{2}{*}{19} & 10 & 3 & 390.3 & 73.3 & 122220001 & -- & 0.4339 \\
\hline & & & & & 222210001 & --- & 0.3609 \\
\hline \multirow[t]{2}{*}{20} & 9 & 1 & 389.5 & 73.4 & 221221000 & 0.0056080 & 0.5974 \\
\hline & & & & & 122220001 & --- & 0.0506 \\
\hline
\end{tabular}


Table S2C-4. Compound 2 - CASSCF/QD-NEVPT2 lowest transition energies with CPCM(THF). 9o, 10e Active Space - 25 triplet roots, 25 singlet roots. Orbitals (in order for CI vector notation below): $\mathrm{d}\left(\mathrm{z}^{2}\right), \mathrm{d}(\mathrm{xz}), \mathrm{d}(\mathrm{xy}), \mathrm{d}(\mathrm{yz})$, bonding $\mathrm{d}\left(\mathrm{x}^{2}-\mathrm{y}^{2}\right) / \mathrm{C}\left(\mathrm{sp}^{2}\right), \mathrm{d}\left(\mathrm{x}^{2}-\mathrm{y}^{2}\right) / \mathrm{C}\left(\mathrm{sp}^{2}\right)^{*}, \pi^{*}(1)$, $\pi^{*}(2), \pi^{*}(3)$.

\begin{tabular}{|c|c|c|c|c|c|c|c|}
\hline State & Root & Multiplicity & $\Delta E / \mathbf{n m}$ & $\Delta \mathrm{E} / \mathrm{kcal} \mathrm{mol}^{-1}$ & CI Vector & $f_{\text {osc }}$ & Contribution \\
\hline \multirow[t]{3}{*}{0} & 0 & 1 & --- & --- & 222220000 & --- & 0.5464 \\
\hline & & & & & 222120100 & --- & 0.1039 \\
\hline & & & & & 222211000 & --- & 0.0853 \\
\hline \multirow[t]{2}{*}{1} & 0 & 3 & 970.1 & 29.5 & 222211000 & $\begin{array}{ll}-- \\
\end{array}$ & 0.4539 \\
\hline & & & & & 122221000 & --- & 0.2912 \\
\hline \multirow[t]{2}{*}{2} & 1 & 3 & 804.6 & 35.5 & 222121000 & --- & 0.6017 \\
\hline & & & & & 212221000 & --- & 0.1400 \\
\hline \multirow[t]{2}{*}{3} & 2 & 3 & 758.2 & 37.7 & 212221000 & --- & 0.5703 \\
\hline & & & & & 222121000 & --- & 0.1536 \\
\hline \multirow[t]{2}{*}{4} & 3 & 3 & 737.1 & 38.8 & 222210100 & --- & 0.5396 \\
\hline & & & & & 122220100 & --- & 0.2880 \\
\hline \multirow[t]{2}{*}{5} & 1 & 1 & 729.4 & 39.2 & 222210100 & 0.0019683 & 0.5703 \\
\hline & & & & & 122220100 & --- & 0.2712 \\
\hline \multirow[t]{2}{*}{6} & 4 & 3 & 642.1 & 44.5 & 222120100 & --- & 0.8142 \\
\hline & & & & & 122112100 & --- & 0.0417 \\
\hline \multirow[t]{2}{*}{7} & 2 & 1 & 624.3 & 45.8 & 222120100 & 0.0905865 & 0.6714 \\
\hline & & & & & 212220100 & --- & 0.0655 \\
\hline \multirow[t]{2}{*}{8} & 5 & 3 & 592.5 & 48.3 & 221221000 & --- & 0.6680 \\
\hline & & & & & 212122000 & --- & 0.0822 \\
\hline \multirow[t]{2}{*}{9} & 6 & 3 & 571.6 & 50.0 & 212220100 & --- & 0.7675 \\
\hline & & & & & 112212100 & --- & 0.0416 \\
\hline \multirow[t]{2}{*}{10} & 3 & 1 & 569.9 & 50.2 & 212220100 & 0.1206524 & 0.6956 \\
\hline & & & & & 212202100 & --- & 0.0386 \\
\hline \multirow[t]{2}{*}{11} & 4 & 1 & 444.4 & 64.3 & 221220100 & 0.0003508 & 0.7306 \\
\hline & & & & & 212121100 & --- & 0.0444 \\
\hline \multirow[t]{2}{*}{12} & 7 & 3 & 433.9 & 65.9 & 222210010 & --- & 0.4812 \\
\hline & & & & & 122220010 & --- & 0.2506 \\
\hline \multirow[t]{2}{*}{13} & 5 & 1 & 432.7 & 66.1 & 222210010 & 0.0010716 & 0.4782 \\
\hline & & & & & 122220010 & --- & 0.2503 \\
\hline \multirow[t]{2}{*}{14} & 8 & 3 & 429.9 & 66.5 & 221220100 & --- & 0.7217 \\
\hline & & & & & 212121100 & --- & 0.0699 \\
\hline \multirow[t]{2}{*}{15} & 6 & 1 & 410.4 & 69.7 & 222120010 & 0.0274877 & 0.3593 \\
\hline & & & & & 222211000 & --- & 0.1504 \\
\hline \multirow[t]{2}{*}{16} & 7 & 1 & 404.5 & 70.7 & 222120001 & 0.0141815 & 0.2505 \\
\hline & & & & & 222211000 & --- & 0.1880 \\
\hline \multirow[t]{2}{*}{17} & 9 & 3 & 400.6 & 71.4 & 222120010 & --- & 0.7918 \\
\hline & & & & & 122112010 & --- & 0.0430 \\
\hline \multirow[t]{2}{*}{18} & 8 & 1 & 399.8 & 71.5 & 222120010 & 0.0076735 & 0.4769 \\
\hline & & & & & 222120010 & --- & 0.0742 \\
\hline \multirow[t]{2}{*}{19} & 9 & 1 & 396.4 & 72.1 & 222210001 & 0.0025346 & 0.3590 \\
\hline & & & & & 122220001 & --- & 0.1937 \\
\hline \multirow[t]{2}{*}{20} & 10 & 3 & 389.0 & 73.5 & 222210001 & --- & 0.5095 \\
\hline & & & & & 122220001 & --- & 0.2709 \\
\hline
\end{tabular}


Table S2D-1. Compound 2 - CASSCF/QD-NEVPT2 composition of the singlet equilibrium ground state in the gas phase. 9o, 10e Active Space - 25 triplet roots, 15 singlet roots. Orbitals: bonding $d\left(x^{2}-y^{2}\right) / C\left(s^{2}\right), d(x y), d(x z), d(y z), d\left(z^{2}\right), d\left(x^{2}-y^{2}\right) / C\left(s^{2}\right)^{*}, \pi^{*}(1), \pi^{*}(2), \pi^{*}(3)$.

\begin{tabular}{llc}
\hline CI Vector & Transition & Contribution \\
\hline 222220000 & Closed shell singlet $(\mathrm{CSS}) \mathrm{d}^{8}$ & 0.5509 \\
222120100 & $\mathrm{~d}(\mathrm{yz}) \rightarrow \pi^{*}(1)$ & 0.1282 \\
122221000 & bonding $\mathrm{d}\left(\mathrm{x}^{2}-\mathrm{y}^{2}\right) / \mathrm{C}\left(\mathrm{sp}^{2}\right) \rightarrow \mathrm{d}\left(\mathrm{x}^{2}-\mathrm{y}^{2}\right) / \mathrm{C}\left(\mathrm{sp}^{2}\right)^{*}$ & 0.0888 \\
222210100 & $\mathrm{~d}\left(\mathrm{z}^{2}\right) \rightarrow \pi^{*}(1)$ & 0.0619 \\
221220001 & $\mathrm{~d}(\mathrm{xz}) \rightarrow \pi^{*}(3)$ & 0.0363 \\
222120010 & $\mathrm{~d}(\mathrm{yz}) \rightarrow \pi^{*}(2)$ & 0.0194 \\
222211000 & $\mathrm{~d}\left(\mathrm{z}^{2}\right) \rightarrow \mathrm{d}\left(\mathrm{x}^{2}-\mathrm{y}^{2}\right) / \mathrm{C}\left(\mathrm{sp}^{2}\right)^{*}$ & 0.0142 \\
022122100 & $2 \mathrm{x}\left[\mathrm{bonding} \mathrm{d}\left(\mathrm{x}^{2}-\mathrm{y}^{2}\right) / \mathrm{C}\left(\mathrm{sp}^{2}\right)\right]+\mathrm{d}(\mathrm{yz}) \rightarrow 2 \mathrm{x}\left[\mathrm{d}\left(\mathrm{x}^{2}-\mathrm{y}^{2}\right) / \mathrm{C}\left(\mathrm{sp}^{2}\right)^{*}\right]+\pi^{*}(1)$ & 0.0134 \\
221220100 & $\mathrm{~d}(\mathrm{xz}) \rightarrow \pi^{*}(1)$ & 0.0075 \\
222202000 & $2 \mathrm{x}\left[\mathrm{d}\left(\mathrm{z}^{2}\right)\right] \rightarrow 2 \mathrm{x}\left[\mathrm{d}\left(\mathrm{x}^{2}-\mathrm{y}^{2}\right) / \mathrm{C}\left(\mathrm{sp}^{2}\right)^{*}\right]$ & 0.0068 \\
022212100 & $2 \mathrm{x}\left[\mathrm{bonding} \mathrm{d}\left(\mathrm{x}^{2}-\mathrm{y}^{2}\right) / \mathrm{C}\left(\mathrm{sp}^{2}\right)\right]+\mathrm{d}\left(\mathrm{z}^{2}\right) \rightarrow 2 \mathrm{x}\left[\mathrm{d}\left(\mathrm{x}^{2}-\mathrm{y}^{2}\right) / \mathrm{C}\left(\mathrm{sp}^{2}\right)^{*}\right]+\pi^{*}(1)$ & 0.0067 \\
220222000 & $2 \mathrm{x}[\mathrm{d}(\mathrm{xz})] \rightarrow 2 \mathrm{x}\left[\mathrm{d}\left(\mathrm{x}^{2}-\mathrm{y}^{2}\right) / \mathrm{C}\left(\mathrm{sp}^{2}\right)^{*}\right]$ & 0.0058 \\
122121100 & $\mathrm{bonding} \mathrm{d}\left(\mathrm{x}^{2}-\mathrm{y}^{2}\right) / \mathrm{C}\left(\mathrm{sp}^{2}\right)+\mathrm{d}(\mathrm{yz})+\rightarrow \mathrm{d}\left(\mathrm{x}^{2}-\mathrm{y}^{2}\right) / \mathrm{C}\left(\mathrm{sp}^{2}\right)^{*}+\pi^{*}(1)$ & 0.0057 \\
222022000 & $2 \mathrm{x}\left[\mathrm{d}(\mathrm{yz}) \rightarrow 2 \mathrm{x}\left[\mathrm{d}\left(\mathrm{x}^{2}-\mathrm{y}^{2}\right) / \mathrm{C}\left(\mathrm{sp}^{2}\right)^{*}\right]\right.$ & 0.0057 \\
222210010 & $\mathrm{~d}\left(\mathrm{z}^{2}\right) \rightarrow \pi^{*}(2)$ & 0.0054 \\
021222001 & $2 \mathrm{x}\left[\right.$ bonding d$\left.\left(\mathrm{x}^{2}-\mathrm{y}^{2}\right) / \mathrm{C}\left(\mathrm{sp}^{2}\right)\right]+\mathrm{d}(\mathrm{xz}) \rightarrow 2 \mathrm{x}\left[\mathrm{d}\left(\mathrm{x}^{2}-\mathrm{y}^{2}\right) / \mathrm{C}\left(\mathrm{sp}^{2}\right)^{*}\right]+\pi^{*}(3)$ & 0.0031 \\
122112100 & bonding d $\left(\mathrm{x}^{2}-\mathrm{y}^{2}\right) / \mathrm{C}\left(\mathrm{sp}^{2}\right)+\mathrm{d}(\mathrm{xz})+\mathrm{d}(\mathrm{yz}) \rightarrow 2 \mathrm{x}\left[\mathrm{d}\left(\mathrm{x}^{2}-\mathrm{y}^{2}\right) / \mathrm{C}\left(\mathrm{sp}^{2}\right)^{*}\right]+\pi^{*}(1)$ & 0.0026 \\
\hline Sum of CSS & & $55 \%$ \\
Sum of all MLCT & & $26 \%$ \\
Sum of all d-d & & $12 \%$ \\
Sum of mixed MLCT $+\mathbf{d}-\mathbf{d}$ & $3.1 \%$ \\
\hline
\end{tabular}

Table S2D-2. Compound 2 - CASSCF/QD-NEVPT2 composition of the singlet equilibrium ground state with CPCM(THF). 9o, 10e Active Space -25 triplet roots, 15 singlet roots. Orbitals: $\mathrm{d}\left(\mathrm{z}^{2}\right), \mathrm{d}(\mathrm{xz}), \mathrm{d}(\mathrm{xy}), \mathrm{d}(\mathrm{yz})$, bonding $\mathrm{d}\left(\mathrm{x}^{2}-\mathrm{y}^{2}\right) / \mathrm{C}\left(\mathrm{sp}^{2}\right), \mathrm{d}\left(\mathrm{x}^{2}-\mathrm{y}^{2}\right) / \mathrm{C}\left(\mathrm{sp}^{2}\right)^{*}, \pi^{*}(1), \pi^{*}(2), \pi^{*}(3)$.

\begin{tabular}{llc}
\hline CI Vector & Transition & Contribution \\
\hline 222220000 & Closed shell singlet $(\mathrm{CSS}) \mathrm{d}^{8}$ & 0.5730 \\
222120100 & $\mathrm{~d}(\mathrm{yz}) \rightarrow \pi^{*}(1)$ & 0.1468 \\
122221000 & bonding $\mathrm{d}\left(\mathrm{x}^{2}-\mathrm{y}^{2}\right) / \mathrm{C}\left(\mathrm{sp}^{2}\right) \rightarrow \mathrm{d}\left(\mathrm{x}^{2}-\mathrm{y}^{2}\right) / \mathrm{C}\left(\mathrm{sp}^{2}\right)^{*}$ & 0.0958 \\
221220001 & $\mathrm{~d}(\mathrm{xz}) \rightarrow \pi^{*}(3)$ & 0.0359 \\
222120010 & $\mathrm{~d}(\mathrm{yz}) \rightarrow \pi^{*}(2)$ & 0.0206 \\
222210100 & $\mathrm{~d}\left(\mathrm{z}^{2}\right) \rightarrow \pi^{*}(1)$ & 0.0178 \\
222211000 & $\mathrm{~d}\left(\mathrm{z}^{2}\right) \rightarrow \mathrm{d}\left(\mathrm{x}^{2}-\mathrm{y}^{2}\right) / \mathrm{C}\left(\mathrm{sp}^{2}\right)^{*}$ & 0.0160 \\
022122100 & $2 \mathrm{x}\left[\mathrm{bonding} \mathrm{d}\left(\mathrm{x}^{2}-\mathrm{y}^{2}\right) / \mathrm{C}\left(\mathrm{sp}^{2}\right)\right]+\mathrm{d}(\mathrm{yz}) \rightarrow 2 \mathrm{x}\left[\mathrm{d}\left(\mathrm{x}^{2}-\mathrm{y}^{2}\right) / \mathrm{C}\left(\mathrm{sp}^{2}\right)^{*}\right]+\pi^{*}(1)$ & 0.0155 \\
221220100 & $\mathrm{~d}(\mathrm{xz}) \rightarrow \pi^{*}(1)$ & 0.0119 \\
222202000 & $2 \mathrm{x}\left[\mathrm{d}\left(\mathrm{z}^{2}\right)\right] \rightarrow 2 \mathrm{x}\left[\mathrm{d}\left(\mathrm{x}^{2}-\mathrm{y}^{2}\right) / \mathrm{C}\left(\mathrm{sp}^{2}\right)^{*}\right]$ & 0.0079 \\
122121100 & $\mathrm{bonding} \mathrm{d}\left(\mathrm{x}^{2}-\mathrm{y}^{2}\right) / \mathrm{C}\left(\mathrm{sp}^{2}\right)+\mathrm{d}(\mathrm{yz})+\rightarrow \mathrm{d}\left(\mathrm{x}^{2}-\mathrm{y}^{2}\right) / \mathrm{C}\left(\mathrm{sp}^{2}\right)^{*}+\pi^{*}(1)$ & 0.0066 \\
220222000 & $2 \mathrm{x}[\mathrm{d}(\mathrm{xz})] \rightarrow 2 \mathrm{x}\left[\mathrm{d}\left(\mathrm{x}^{2}-\mathrm{y}^{2}\right) / \mathrm{C}\left(\mathrm{sp}^{2}\right)^{*}\right]$ & 0.0063 \\
222022000 & $2 \mathrm{x}[\mathrm{d}(\mathrm{yz})] \rightarrow 2 \mathrm{x}\left[\mathrm{d}\left(\mathrm{x}^{2}-\mathrm{y}^{2}\right) / \mathrm{C}\left(\mathrm{sp}^{2}\right)^{*}\right]$ & 0.0059 \\
021222001 & $2 \mathrm{x}\left[\mathrm{bonding} \mathrm{d}\left(\mathrm{x}^{2}-\mathrm{y}^{2}\right) / \mathrm{C}\left(\mathrm{sp}^{2}\right)\right]+\mathrm{d}(\mathrm{xz}) \rightarrow 2 \mathrm{x}\left[\mathrm{d}\left(\mathrm{x}^{2}-\mathrm{y}^{2}\right) / \mathrm{C}\left(\mathrm{sp}^{2}\right)^{*}\right]+\pi^{*}(3)$ & 0.0030 \\
122112100 & $\mathrm{bonding} \mathrm{d}\left(\mathrm{x}^{2}-\mathrm{y}^{2}\right) / \mathrm{C}\left(\mathrm{sp}^{2}\right)+\mathrm{d}(\mathrm{yz})+\mathrm{d}\left(\mathrm{z}^{2}\right) \rightarrow 2 \mathrm{x}\left[\mathrm{d}\left(\mathrm{x}^{2}-\mathrm{y}^{2}\right) / \mathrm{C}\left(\mathrm{sp}^{2}\right)^{*}\right]+\pi^{*}(1)$ & 0.0025 \\
\hline Sum of CSS & & $57 \%$ \\
Sum of all MLCT & $23 \%$ \\
Sum of all d-d & & $13 \%$ \\
Sum of mixed MLCT $+\mathbf{d}-\mathbf{d}$ & $2.8 \%$ \\
\hline
\end{tabular}


Table S2D-3. Compound 2 - CASSCF/QD-NEVPT2 composition of the singlet equilibrium ground state in gas phase. $9 \mathrm{o}, 10 \mathrm{e}$ Active Space -25 triplet roots, 25 singlet roots. Orbitals: $\mathrm{d}\left(\mathrm{z}^{2}\right)$, $\mathrm{d}(\mathrm{xz}), \mathrm{d}(\mathrm{xy}), \mathrm{d}(\mathrm{yz})$, bonding $\mathrm{d}\left(\mathrm{x}^{2}-\mathrm{y}^{2}\right) / \mathrm{C}\left(\mathrm{sp}^{2}\right), \mathrm{d}\left(\mathrm{x}^{2}-\mathrm{y}^{2}\right) / \mathrm{C}\left(\mathrm{sp}^{2}\right)^{*}, \pi^{*}(1), \pi^{*}(2), \pi^{*}(3)$.

\begin{tabular}{llc}
\hline CI Vector & Transition & Contribution \\
\hline 222220000 & Closed shell singlet $(\mathrm{CSS}) \mathrm{d}^{8}$ & 0.5529 \\
222211000 & bonding $\mathrm{d}\left(\mathrm{x}^{2}-\mathrm{y}^{2}\right) / \mathrm{C}\left(\mathrm{sp}^{2}\right) \rightarrow \mathrm{d}\left(\mathrm{x}^{2}-\mathrm{y}^{2}\right) / \mathrm{C}\left(\mathrm{sp}^{2}\right)^{*}$ & 0.1358 \\
222120100 & $\mathrm{~d}(\mathrm{yz}) \rightarrow \pi^{*}(1)$ & 0.0884 \\
122221000 & $\mathrm{~d}\left(\mathrm{z}^{2}\right) \rightarrow \mathrm{d}\left(\mathrm{x}^{2}-\mathrm{y}^{2}\right) / \mathrm{C}\left(\mathrm{sp}^{2}\right)^{*}$ & 0.0547 \\
212220100 & $\mathrm{~d}(\mathrm{xz}) \rightarrow \pi^{*}(1)$ & 0.0274 \\
222120010 & $\mathrm{~d}(\mathrm{yz}) \rightarrow \pi^{*}(2)$ & 0.0265 \\
212220001 & $\mathrm{~d}(\mathrm{xz}) \rightarrow \pi^{*}(3)$ & 0.0246 \\
122212000 & $\mathrm{~d}\left(\mathrm{z}^{2}\right)+$ bonding $\mathrm{d}\left(\mathrm{x}^{2}-\mathrm{y}^{2}\right) / \mathrm{C}\left(\mathrm{sp}^{2}\right) \rightarrow 2 \mathrm{x}\left[\mathrm{d}\left(\mathrm{x}^{2}-\mathrm{y}^{2}\right) / \mathrm{C}\left(\mathrm{sp}^{2}\right)^{*}\right]$ & 0.0122 \\
202222000 & $2 \mathrm{x}[\mathrm{d}(\mathrm{xz})] \rightarrow 2 \mathrm{x}\left[\mathrm{d}\left(\mathrm{x}^{2}-\mathrm{y}^{2}\right) / \mathrm{C}\left(\mathrm{sp}^{2}\right)^{*}\right]$ & 0.0070 \\
222022000 & $2 \mathrm{x}[\mathrm{d}(\mathrm{yz})] \rightarrow 2 \mathrm{x}\left[\mathrm{d}\left(\mathrm{x}^{2}-\mathrm{y}^{2}\right) / \mathrm{C}\left(\mathrm{sp}^{2}\right)^{*}\right]$ & 0.0069 \\
222102100 & $\mathrm{~d}(\mathrm{yz})+2 \mathrm{x}\left[\mathrm{bonding} \mathrm{d}\left(\mathrm{x}^{2}-\mathrm{y}^{2}\right) / \mathrm{C}\left(\mathrm{sp}^{2}\right)\right] \rightarrow 2 \mathrm{x}\left[\mathrm{d}\left(\mathrm{x}^{2}-\mathrm{y}^{2}\right) / \mathrm{C}\left(\mathrm{sp}^{2}\right)^{*}\right]+\pi^{*}(1)$ & 0.0058 \\
122220100 & $\mathrm{~d}\left(\mathrm{z}^{2}\right) \rightarrow \pi^{*}(1)$ & 0.0056 \\
122112100 & $\mathrm{~d}\left(\mathrm{z}^{2}\right)+\mathrm{d}(\mathrm{yz})+\mathrm{bonding} \mathrm{d}\left(\mathrm{x}^{2}-\mathrm{y}^{2}\right) / \mathrm{C}\left(\mathrm{sp}^{2}\right) \rightarrow 2 \mathrm{x}\left[\mathrm{d}\left(\mathrm{x}^{2}-\mathrm{y}^{2}\right) / \mathrm{C}\left(\mathrm{sp}^{2}\right)^{*}\right]+\pi^{*}(1)$ & 0.0052 \\
222120001 & $\mathrm{~d}(\mathrm{yz}) \rightarrow \pi^{*}(3)$ & 0.0051 \\
222210100 & $\mathrm{bonding} \mathrm{d}\left(\mathrm{x}^{2}-\mathrm{y}^{2}\right) / \mathrm{C}\left(\mathrm{sp}^{2}\right) \rightarrow \pi^{*}(1)$ & 0.0039 \\
022222000 & $2 \mathrm{x}\left[\mathrm{d}\left(\mathrm{z}^{2}\right)\right] \rightarrow 2 \mathrm{x}\left[\mathrm{d}\left(\mathrm{x}^{2}-\mathrm{y}^{2}\right) / \mathrm{C}\left(\mathrm{sp}^{2}\right)^{*}\right]$ & 0.0025 \\
\hline Sum of CSS & & $55 \%$ \\
Sum of all MLCT & & $18 \%$ \\
Sum of all d-d & & $21 \%$ \\
Sum of mixed MLCT $+\mathrm{d}-\mathbf{d}$ & $2.3 \%$ \\
\hline
\end{tabular}

Table S2D-4. Compound 2 - CASSCF/QD-NEVPT2 composition of the singlet equilibrium ground state with CPCM(THF). 9o, 10e Active Space - 25 triplet roots, 25 singlet roots. Orbitals: $\mathrm{d}\left(\mathrm{z}^{2}\right), \mathrm{d}(\mathrm{xz}), \mathrm{d}(\mathrm{xy}), \mathrm{d}(\mathrm{yz})$, bonding $\mathrm{d}\left(\mathrm{x}^{2}-\mathrm{y}^{2}\right) / \mathrm{C}\left(\mathrm{sp}^{2}\right), \mathrm{d}\left(\mathrm{x}^{2}-\mathrm{y}^{2}\right) / \mathrm{C}\left(\mathrm{sp}^{2}\right)^{*}, \pi^{*}(1), \pi^{*}(2), \pi^{*}(3)$.

\begin{tabular}{llc}
\hline CI Vector & Transition & Contribution \\
\hline 222220000 & Closed shell singlet $(\mathrm{CSS}) \mathrm{d}^{8}$ & 0.5464 \\
222120100 & $\mathrm{~d}(\mathrm{yz}) \rightarrow \pi^{*}(1)$ & 0.1039 \\
122221000 & $\mathrm{~d}\left(\mathrm{z}^{2}\right) \rightarrow \mathrm{d}\left(\mathrm{x}^{2}-\mathrm{y}^{2}\right)$ & 0.0858 \\
222211000 & bonding $\mathrm{d}\left(\mathrm{x}^{2}-\mathrm{y}^{2}\right) / \mathrm{C}\left(\mathrm{sp}^{2}\right) \rightarrow \mathrm{d}\left(\mathrm{x}^{2}-\mathrm{y}^{2}\right) / \mathrm{C}\left(\mathrm{sp}^{2}\right)^{*}$ & 0.0853 \\
212220100 & $\mathrm{~d}(\mathrm{xz}) \rightarrow \pi^{*}(1)$ & 0.0373 \\
212220001 & $\mathrm{~d}(\mathrm{xz}) \rightarrow \pi^{*}(3)$ & 0.0290 \\
222120010 & $\mathrm{~d}(\mathrm{yz}) \rightarrow \pi^{*}(2)$ & 0.0236 \\
122212000 & $\mathrm{~d}\left(\mathrm{z}^{2}\right)+$ bonding $\mathrm{d}\left(\mathrm{x}^{2}-\mathrm{y}^{2}\right) / \mathrm{C}\left(\mathrm{sp}^{2}\right) \rightarrow 2 \mathrm{x}\left[\mathrm{d}\left(\mathrm{x}^{2}-\mathrm{y}^{2}\right) / \mathrm{C}\left(\mathrm{sp}^{2}\right)^{*}\right]$ & 0.0122 \\
202222000 & $2 \mathrm{x}[\mathrm{d}(\mathrm{xz})] \rightarrow 2 \mathrm{x}\left[\mathrm{d}\left(\mathrm{x}^{2}-\mathrm{y}^{2}\right) / \mathrm{C}\left(\mathrm{sp}^{2}\right)^{*}\right]$ & 0.0069 \\
222022000 & $2 \mathrm{x}[\mathrm{d}(\mathrm{yz})] \rightarrow 2 \mathrm{x}\left[\mathrm{d}\left(\mathrm{x}^{2}-\mathrm{y}^{2}\right) / C\left(\mathrm{sp}^{2}\right)^{*}\right]$ & 0.0066 \\
122112100 & $\mathrm{~d}\left(\mathrm{z}^{2}\right)+\mathrm{d}(\mathrm{yz})+$ bonding $\mathrm{d}\left(\mathrm{x}^{2}-\mathrm{y}^{2}\right) / \mathrm{C}\left(\mathrm{sp}^{2}\right) \rightarrow 2 \mathrm{x}\left[\mathrm{d}\left(\mathrm{x}^{2}-\mathrm{y}^{2}\right) / \mathrm{C}\left(\mathrm{sp}^{2}\right)^{*}\right]+\pi^{*}(1)$ & 0.0063 \\
222120001 & $\mathrm{~d}(\mathrm{yz}) \rightarrow \pi^{*}(3)$ & 0.0056 \\
022122100 & $\mathrm{~d}\left(\mathrm{z}^{2}\right)+\mathrm{d}(\mathrm{yz}) \rightarrow \mathrm{d}\left(\mathrm{x}^{2}-\mathrm{y}^{2}\right)+\pi^{*}(1)$ & 0.0054 \\
222210100 & $\mathrm{bonding} \mathrm{d}\left(\mathrm{x}^{2}-\mathrm{y}^{2}\right) / \mathrm{C}\left(\mathrm{sp}^{2}\right) \rightarrow \pi^{*}(1)$ & 0.0053 \\
222102100 & $\mathrm{~d}(\mathrm{yz})+$ bonding d$\left(\mathrm{x}^{2}-\mathrm{y}^{2}\right) / \mathrm{C}\left(\mathrm{sp}^{2}\right) \rightarrow \mathrm{d}\left(\mathrm{x}^{2}-\mathrm{y}^{2}\right) / \mathrm{C}\left(\mathrm{sp}^{2}\right)^{*}+\pi^{*}(1)$ & 0.0030 \\
\hline Sum of CSS & & $55 \%$ \\
Sum of all MLCT & $20 \%$ \\
Sum of all d-d & & $20 \%$ \\
Sum of mixed MLCT $+\mathbf{d}-\mathbf{d}$ & $1.5 \%$ \\
\hline
\end{tabular}


Table S2E-1. Compound 2 - CASSCF/QD-NEVPT2 lowest transition energies in the gas phase. 10o, 12e Active Space - 20 triplet roots, 10 singlet roots. Orbitals (in order for CI vector notation below): bonding $\mathrm{d}(\mathrm{xy}) / \mathrm{C}(\pi)$, bonding $\mathrm{d}\left(\mathrm{x}^{2}-\mathrm{y}^{2}\right) / \mathrm{C}\left(\mathrm{sp}^{2}\right), \mathrm{d}(\mathrm{xz}), \mathrm{d}(\mathrm{xy}), \mathrm{d}(\mathrm{yz}), \mathrm{d}\left(\mathrm{z}^{2}\right), \mathrm{d}\left(\mathrm{x}^{2}-\mathrm{y}^{2}\right) / \mathrm{C}\left(\mathrm{sp}^{2}\right)^{*}$, $\pi^{*}(1), \pi^{*}(2), \pi^{*}(3)$.

\begin{tabular}{|c|c|c|c|c|c|c|c|}
\hline State & Root & Multiplicity & $\Delta E / n m$ & $\Delta \mathrm{E} / \mathrm{kcal} \mathrm{mol}^{-1}$ & CI Vector & $f_{\text {osc }}$ & Contribution \\
\hline \multirow[t]{3}{*}{0} & 0 & 1 & --- & --- & 2222220000 & --- & 0.6318 \\
\hline & & & & & 2222120100 & --- & 0.1391 \\
\hline & & & & & 2122221000 & --- & 0.0531 \\
\hline \multirow[t]{2}{*}{1} & 0 & 3 & 1000.6 & 28.6 & 2222210100 & $\begin{array}{l}-- \\
\end{array}$ & 0.8429 \\
\hline & & & & & 2022212100 & -- & 0.0675 \\
\hline \multirow[t]{2}{*}{2} & 1 & 1 & 960.9 & 29.8 & 2222210100 & 0.0021210 & 0.8218 \\
\hline & & & & & 2022212100 & & 0.0667 \\
\hline \multirow[t]{2}{*}{3} & 1 & 3 & 846.1 & 33.8 & 2222120100 & --- & 0.7748 \\
\hline & & & & & 2022122100 & --- & 0.0698 \\
\hline \multirow[t]{2}{*}{4} & 2 & 1 & 775.8 & 36.9 & 2212220100 & 0.0192175 & 0.5127 \\
\hline & & & & & 2222120100 & & 0.2761 \\
\hline \multirow[t]{2}{*}{5} & 2 & 3 & 738.0 & 38.7 & 2212220100 & --- & 0.7239 \\
\hline & & & & & 2012222100 & --- & 0.0538 \\
\hline \multirow[t]{2}{*}{6} & 3 & 3 & 714.1 & 40.0 & 2222211000 & --- & 0.7712 \\
\hline & & & & & 2122212000 & --- & 0.0802 \\
\hline \multirow[t]{2}{*}{7} & 4 & 3 & 626.0 & 45.7 & 2212221000 & --- & 0.5501 \\
\hline & & & & & 2222121000 & --- & 0.2572 \\
\hline \multirow[t]{2}{*}{8} & 3 & 1 & 619.0 & 46.2 & 2222120100 & 0.2167547 & 0.3590 \\
\hline & & & & & 2212220100 & & 0.2585 \\
\hline \multirow[t]{2}{*}{9} & 5 & 3 & 577.6 & 49.5 & 2222121000 & --- & 0.5793 \\
\hline & & & & & 2212221000 & --- & 0.2374 \\
\hline \multirow[t]{2}{*}{10} & 4 & 1 & 560.2 & 51.0 & 2221220100 & 0.0023581 & 0.7622 \\
\hline & & & & & 2212121100 & & 0.0553 \\
\hline \multirow[t]{2}{*}{11} & 6 & 3 & 541.8 & 52.8 & 2221220100 & $\begin{array}{l}-- \\
\end{array}$ & 0.7308 \\
\hline & & & & & 2212121100 & -- & 0.0550 \\
\hline \multirow[t]{2}{*}{12} & 7 & 3 & 524.8 & 54.5 & 2221221000 & --- & 0.6806 \\
\hline & & & & & 2221121100 & --- & 0.0483 \\
\hline \multirow[t]{2}{*}{13} & 8 & 3 & 455.1 & 62.8 & 2222210010 & --- & 0.4270 \\
\hline & & & & & 2222210001 & --- & 0.4167 \\
\hline \multirow[t]{2}{*}{14} & 5 & 1 & 433.8 & 65.9 & 2222210010 & 0.0146952 & 0.7914 \\
\hline & & & & & 2022212010 & & 0.0650 \\
\hline \multirow[t]{2}{*}{15} & 9 & 3 & 432.4 & 66.1 & 2222210001 & --- & 0.4282 \\
\hline & & & & & 2222210010 & --- & 0.4100 \\
\hline \multirow[t]{2}{*}{16} & 10 & 3 & 405.7 & 70.5 & 2222120010 & --- & 0.5858 \\
\hline & & & & & 2212220010 & -- & 0.1251 \\
\hline \multirow[t]{2}{*}{17} & 6 & 1 & 391.2 & 73.1 & 2221221000 & 0.0017318 & 0.6850 \\
\hline & & & & & 2222211000 & & 0.0873 \\
\hline \multirow[t]{2}{*}{18} & 7 & 1 & 373.8 & 76.5 & 2222211000 & 0.0037102 & 0.7321 \\
\hline & & & & & 2221221000 & & 0.0891 \\
\hline \multirow[t]{2}{*}{19} & 8 & 1 & 364.5 & 78.4 & 2222121000 & 0.0007688 & 0.4732 \\
\hline & & & & & 2212221000 & & 0.3645 \\
\hline \multirow[t]{2}{*}{20} & 11 & 3 & 357.3 & 80.0 & 2212220010 & --- & 0.4398 \\
\hline & & & & & 2222120001 & -- & 0.1767 \\
\hline
\end{tabular}


Table S2E-2. Compound 2 - CASSCF/QD-NEVPT2 lowest transition energies in the gas phase. 10o, 12e Active Space - 25 triplet roots, 15 singlet roots. Orbitals (in order for CI vector notation below): bonding $\mathrm{d}(\mathrm{xy}) / \mathrm{C}(\pi)$, bonding $\mathrm{d}\left(\mathrm{x}^{2}-\mathrm{y}^{2}\right) / \mathrm{C}\left(\mathrm{sp}^{2}\right), \mathrm{d}(\mathrm{xy}), \mathrm{d}(\mathrm{xz}), \mathrm{d}(\mathrm{yz}), \mathrm{d}\left(\mathrm{z}^{2}\right), \mathrm{d}\left(\mathrm{x}^{2}-\mathrm{y}^{2}\right) / \mathrm{C}\left(\mathrm{sp}^{2}\right)^{*}$, $\pi^{*}(1), \pi^{*}(2), \pi^{*}(3)$.

\begin{tabular}{|c|c|c|c|c|c|c|c|}
\hline State & Root & Multiplicity & $\Delta \mathrm{E} / \mathbf{n m}$ & $\Delta \mathrm{E} / \mathrm{kcal} \mathrm{mol}^{-1}$ & CI Vector & $f_{\text {osc }}$ & Contribution \\
\hline \multirow[t]{3}{*}{0} & 0 & 1 & --- & --- & 2222220000 & -- & 0.5796 \\
\hline & & & & & 2222120100 & --- & 0.1775 \\
\hline & & & & & 2122221000 & --- & 0.0644 \\
\hline \multirow[t]{2}{*}{1} & 0 & 3 & 922.3 & 31.0 & 2222210100 & --- & 0.8168 \\
\hline & & & & & 2022212100 & --- & 0.0659 \\
\hline \multirow[t]{2}{*}{2} & 1 & 1 & 890.7 & 32.1 & 2222210100 & 0.00179 & 0.7846 \\
\hline & & & & & 2022212100 & --- & 0.0631 \\
\hline \multirow[t]{2}{*}{3} & 1 & 3 & 809.4 & 35.3 & 2222211000 & --- & 0.4160 \\
\hline & & & & & 2222120100 & --- & 0.3302 \\
\hline \multirow[t]{2}{*}{4} & 2 & 3 & 778.7 & 36.7 & 2222120100 & --- & 0.4159 \\
\hline & & & & & 2222211000 & --- & 0.3457 \\
\hline \multirow[t]{2}{*}{5} & 2 & 1 & 720.5 & 39.7 & 2221220100 & 0.03340 & 0.5256 \\
\hline & & & & & 2222120100 & --- & 0.2310 \\
\hline \multirow[t]{2}{*}{6} & 3 & 3 & 686.7 & 41.6 & 2221221000 & --- & 0.4526 \\
\hline & & & & & 2222121000 & --- & 0.3029 \\
\hline \multirow[t]{2}{*}{7} & 4 & 3 & 675.3 & 42.3 & 2221220100 & --- & 0.7063 \\
\hline & & & & & 2021222100 & --- & 0.0498 \\
\hline \multirow[t]{2}{*}{8} & 5 & 3 & 638.2 & 44.8 & 2222121000 & --- & 0.4856 \\
\hline & & & & & 2221221000 & --- & 0.3037 \\
\hline \multirow[t]{2}{*}{9} & 3 & 1 & 632.0 & 45.2 & 2222120100 & 0.17525 & 0.3036 \\
\hline & & & & & 2221220100 & --- & 0.2534 \\
\hline \multirow[t]{2}{*}{10} & 6 & 3 & 549.5 & 52.0 & 2212221000 & --- & 0.6054 \\
\hline & & & & & 2212121100 & --- & 0.0685 \\
\hline \multirow[t]{2}{*}{11} & 4 & 1 & 517.8 & 55.2 & 2212220100 & 0.00051 & 0.7254 \\
\hline & & & & & 2221121100 & --- & 0.0611 \\
\hline \multirow[t]{2}{*}{12} & 7 & 3 & 509.9 & 56.1 & 2212220100 & --- & 0.7244 \\
\hline & & & & & 2221121100 & --- & 0.0600 \\
\hline \multirow[t]{2}{*}{13} & 8 & 3 & 489.3 & 58.4 & 2222210010 & --- & 0.7691 \\
\hline & & & & & 2022212010 & --- & 0.0670 \\
\hline \multirow[t]{2}{*}{14} & 5 & 1 & 487.5 & 58.6 & 2222210010 & 0.00192 & 0.7185 \\
\hline & & & & & 2022212010 & --- & 0.0627 \\
\hline \multirow[t]{2}{*}{15} & 6 & 1 & 458.8 & 62.3 & 2222120010 & 0.09519 & 0.4058 \\
\hline & & & & & 2221220010 & --- & 0.1885 \\
\hline \multirow[t]{2}{*}{16} & 9 & 3 & 452.2 & 63.2 & 2222120010 & --- & 0.7398 \\
\hline & & & & & 2022122010 & --- & 0.0698 \\
\hline \multirow[t]{2}{*}{17} & 7 & 1 & 442.8 & 64.6 & 2222210001 & 0.01268 & 0.7254 \\
\hline & & & & & 2022212001 & --- & 0.0617 \\
\hline \multirow[t]{2}{*}{18} & 10 & 3 & 430.1 & 66.5 & 2222210001 & --- & 0.7692 \\
\hline & & & & & 2022212001 & --- & 0.0651 \\
\hline \multirow[t]{2}{*}{19} & 8 & 1 & 430.0 & 66.5 & 2221220010 & 0.00571 & 0.5576 \\
\hline & & & & & 2222120010 & --- & 0.1065 \\
\hline \multirow[t]{2}{*}{20} & 11 & 3 & 409.9 & 69.7 & 2221220010 & --- & 0.6892 \\
\hline & & & & & 2221220001 & --- & 0.0620 \\
\hline
\end{tabular}


Table S2E-3. Compound 2 - CASSCF/QD-NEVPT2 Lowest transition energies with CPCM(THF). 10o, 12e Active Space - 25 triplet roots, 15 singlet roots. Orbitals (in order for CI vector notation below): bonding $\mathrm{d}(\mathrm{xy}) / \mathrm{C}(\pi)$, bonding $\mathrm{d}\left(\mathrm{x}^{2}-\mathrm{y}^{2}\right) / \mathrm{C}\left(\mathrm{sp}^{2}\right), \mathrm{d}(\mathrm{xy}), \mathrm{d}(\mathrm{xz}), \mathrm{d}(\mathrm{yz}), \mathrm{d}\left(\mathrm{z}^{2}\right)$, $\mathrm{d}\left(\mathrm{x}^{2}-\mathrm{y}^{2}\right) / \mathrm{C}\left(\mathrm{sp}^{2}\right)^{*}, \pi^{*}(1), \pi^{*}(2), \pi^{*}(3)$.

\begin{tabular}{|c|c|c|c|c|c|c|c|}
\hline State & Root & Multiplicity & $\Delta \mathbf{E} / \mathbf{n m}$ & $\Delta \mathrm{E} / \mathrm{kcal} \mathrm{mol}^{-1}$ & CI Vector & $f_{\text {osc }}$ & Contribution \\
\hline \multirow[t]{3}{*}{0} & 0 & 1 & --- & --- & 2222220000 & -- & 0.6012 \\
\hline & & & & & 2222120100 & --- & 0.1574 \\
\hline & & & & & 2122221000 & --- & 0.0715 \\
\hline \multirow[t]{2}{*}{1} & 0 & 3 & 907.8 & 31.5 & 2222210100 & --- & 0.7864 \\
\hline & & & & & 2022212100 & --- & 0.0647 \\
\hline \multirow[t]{2}{*}{2} & 1 & 3 & 816.4 & 35.0 & 2222211000 & --- & 0.7763 \\
\hline & & & & & 2122212000 & --- & 0.0980 \\
\hline \multirow[t]{2}{*}{3} & 1 & 1 & 805.7 & 35.5 & 2222210100 & 0.0015632 & 0.7932 \\
\hline & & & & & 2022212100 & --- & 0.0651 \\
\hline \multirow[t]{2}{*}{4} & 2 & 3 & 717.2 & 39.9 & 2222120100 & $\begin{array}{ll}-- \\
\end{array}$ & 0.7553 \\
\hline & & & & & 2022122100 & --- & 0.0679 \\
\hline \multirow[t]{2}{*}{5} & 2 & 1 & 666.5 & 42.9 & 2221220100 & 0.0318143 & 0.5075 \\
\hline & & & & & 2222120100 & --- & 0.2601 \\
\hline \multirow[t]{2}{*}{6} & 3 & 3 & 656.2 & 43.6 & 2222121000 & --- & 0.3972 \\
\hline & & & & & 2221221000 & --- & 0.3808 \\
\hline \multirow[t]{2}{*}{7} & 4 & 3 & 652.4 & 43.8 & 2222121000 & --- & 0.4020 \\
\hline & & & & & 2221221000 & --- & 0.3910 \\
\hline \multirow[t]{2}{*}{8} & 5 & 3 & 633.5 & 45.1 & 2221220100 & --- & 0.7386 \\
\hline & & & & & 2222120100 & --- & 0.0451 \\
\hline \multirow[t]{2}{*}{9} & 3 & 1 & 584.7 & 48.9 & 2222120100 & 0.1984600 & 0.3109 \\
\hline & & & & & 2221220100 & --- & 0.2682 \\
\hline \multirow[t]{2}{*}{10} & 6 & 3 & 547.0 & 52.3 & 2212221000 & --- & 0.6240 \\
\hline & & & & & 2221122000 & --- & 0.0672 \\
\hline \multirow[t]{2}{*}{11} & 4 & 1 & 487.9 & 58.6 & 2212220100 & 0.0005989 & 0.7323 \\
\hline & & & & & 2221121100 & --- & 0.0615 \\
\hline \multirow[t]{2}{*}{12} & 7 & 3 & 477.5 & 59.9 & 2212220100 & --- & 0.7351 \\
\hline & & & & & 2221121100 & --- & 0.0610 \\
\hline \multirow[t]{2}{*}{13} & 8 & 3 & 460.5 & 62.1 & 2222210010 & --- & 0.6676 \\
\hline & & & & & 2222210001 & --- & 0.1412 \\
\hline \multirow[t]{2}{*}{14} & 5 & 1 & 456.2 & 62.7 & 2222210010 & 0.0029045 & 0.7120 \\
\hline & & & & & 2022212010 & --- & 0.0633 \\
\hline \multirow[t]{2}{*}{15} & 6 & 1 & 443.5 & 64.5 & 2222120010 & 0.1027144 & 0.4285 \\
\hline & & & & & 2221220010 & --- & 0.1113 \\
\hline \multirow[t]{2}{*}{16} & 9 & 3 & 429.7 & 66.5 & 2222210001 & --- & 0.6684 \\
\hline & & & & & 2222210010 & --- & 0.1350 \\
\hline \multirow[t]{2}{*}{17} & 10 & 3 & 424.1 & 67.4 & 2222120010 & --- & 0.7429 \\
\hline & & & & & 2122121010 & --- & 0.0501 \\
\hline \multirow[t]{2}{*}{18} & 7 & 1 & 410.1 & 69.7 & 2222210001 & 0.0097454 & 0.6190 \\
\hline & & & & & 2222211000 & --- & 0.0582 \\
\hline \multirow[t]{2}{*}{19} & 8 & 1 & 398.6 & 71.7 & 2212221000 & 0.0169288 & 0.3458 \\
\hline & & & & & 2222210001 & --- & 0.1354 \\
\hline \multirow[t]{2}{*}{20} & 11 & 3 & 391.6 & 73.0 & 2221220010 & --- & 0.7063 \\
\hline & & & & & 2221220001 & --- & 0.0557 \\
\hline
\end{tabular}


Table S2E-4. Compound 2 - CASSCF/QD-NEVPT2 lowest transition energies in the gas phase. 10o, 12e Active Space - 40 triplet roots, 25 singlet roots. Orbitals (in order for CI vector notation below): bonding $\mathrm{d}(\mathrm{xy}) / \mathrm{C}(\pi)$, bonding $\mathrm{d}\left(\mathrm{x}^{2}-\mathrm{y}^{2}\right) / \mathrm{C}\left(\mathrm{sp}^{2}\right), \mathrm{d}(\mathrm{xz}), \mathrm{d}(\mathrm{yz}), \mathrm{d}\left(\mathrm{z}^{2}\right), \mathrm{d}(\mathrm{xy}), \mathrm{d}\left(\mathrm{x}^{2}-\mathrm{y}^{2}\right) / \mathrm{C}\left(\mathrm{sp}^{2}\right)^{*}$, $\pi^{*}(1), \pi^{*}(2), \pi^{*}(3)$.

\begin{tabular}{|c|c|c|c|c|c|c|c|}
\hline State & Root & Multiplicity & $\Delta \mathbf{E} / \mathbf{n m}$ & $\Delta \mathrm{E} / \mathrm{kcal} \mathrm{mol}^{-1}$ & CI Vector & $f_{\text {osc }}$ & Contribution \\
\hline \multirow[t]{3}{*}{0} & 0 & 1 & --- & --- & 2222220000 & -- & 0.6252 \\
\hline & & & & & 2221220100 & --- & 0.0660 \\
\hline & & & & & 2212220100 & --- & 0.0637 \\
\hline \multirow[t]{2}{*}{1} & 0 & 3 & 846.7 & 33.8 & 2222121000 & --- & 0.5072 \\
\hline & & & & & 2122221000 & --- & 0.1444 \\
\hline \multirow[t]{2}{*}{2} & 1 & 3 & 791.3 & 36.1 & 2222120100 & 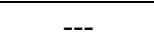 & 0.5010 \\
\hline & & & & & 2222210100 & -- & 0.1672 \\
\hline \multirow[t]{2}{*}{3} & 1 & 1 & 770.4 & 37.1 & 2222120100 & 0.0024769 & 0.5080 \\
\hline & & & & & 2222210100 & --- & 0.1611 \\
\hline \multirow[t]{2}{*}{4} & 2 & 3 & 754.4 & 37.9 & 2221221000 & --- & 0.7900 \\
\hline & & & & & 2121222000 & --- & 0.0728 \\
\hline \multirow[t]{2}{*}{5} & 3 & 3 & 706.9 & 40.4 & 2212221000 & --- & 0.6698 \\
\hline & & & & & 2122221000 & --- & 0.0929 \\
\hline \multirow[t]{2}{*}{6} & 4 & 3 & 691.6 & 41.3 & 2221220100 & --- & 0.7037 \\
\hline & & & & & 2212220100 & --- & 0.0610 \\
\hline \multirow[t]{2}{*}{7} & 2 & 1 & 660.5 & 43.3 & 2221220100 & 0.0915113 & 0.6732 \\
\hline & & & & & 2222220000 & --- & 0.0387 \\
\hline \multirow[t]{2}{*}{8} & 5 & 3 & 622.2 & 46.0 & 2212220100 & --- & 0.6105 \\
\hline & & & & & 2122220100 & --- & 0.0586 \\
\hline \multirow[t]{2}{*}{9} & 3 & 1 & 598.3 & 47.8 & 2212220100 & 0.0987239 & 0.5993 \\
\hline & & & & & 2122220100 & --- & 0.0634 \\
\hline \multirow[t]{2}{*}{10} & 6 & 3 & 573.0 & 49.9 & 2222211000 & --- & 0.4666 \\
\hline & & & & & 2222121000 & --- & 0.1321 \\
\hline \multirow[t]{2}{*}{11} & 4 & 1 & 476.0 & 60.1 & 2222210100 & 0.0009262 & 0.4903 \\
\hline & & & & & 2222120100 & --- & 0.2030 \\
\hline \multirow[t]{2}{*}{12} & 7 & 3 & 473.2 & 60.4 & 2222210100 & --- & 0.5038 \\
\hline & & & & & 2222120100 & --- & 0.2033 \\
\hline \multirow[t]{2}{*}{13} & 8 & 3 & 456.7 & 62.6 & 2222120010 & $\begin{array}{l}-- \\
\end{array}$ & 0.4563 \\
\hline & & & & & 2222210010 & --- & 0.1511 \\
\hline \multirow[t]{2}{*}{14} & 5 & 1 & 454.8 & 62.9 & 2222120010 & 0.0020837 & 0.4470 \\
\hline & & & & & 2222210010 & --- & 0.1435 \\
\hline \multirow[t]{2}{*}{15} & 6 & 1 & 428.3 & 66.8 & 2221220010 & 0.0399342 & 0.4974 \\
\hline & & & & & 2221220001 & --- & 0.2063 \\
\hline \multirow[t]{2}{*}{16} & 9 & 3 & 424.5 & 67.4 & 2221220010 & --- & 0.6741 \\
\hline & & & & & 2212220010 & --- & 0.0759 \\
\hline \multirow[t]{2}{*}{17} & 7 & 1 & 407.4 & 70.2 & 2222120001 & 0.0152654 & 0.4264 \\
\hline & & & & & 2122220001 & --- & 0.1124 \\
\hline \multirow[t]{2}{*}{18} & 10 & 3 & 403.2 & 70.9 & 2222120001 & --- & 0.4598 \\
\hline & & & & & 2222210001 & --- & 0.1477 \\
\hline \multirow[t]{2}{*}{19} & 8 & 1 & 399.7 & 71.5 & 2221220001 & 0.1277239 & 0.2755 \\
\hline & & & & & 2212220001 & --- & 0.1806 \\
\hline \multirow[t]{2}{*}{20} & 9 & 1 & 395.7 & 72.3 & 2221221000 & 0.0091248 & 0.3478 \\
\hline & & & & & 2212220010 & --- & 0.1772 \\
\hline
\end{tabular}


Table S2F-1. Compound 2 - CASSCF/QD-NEVPT2 composition of the singlet equilibrium ground state in the gas phase. 10o, 12e Active Space - 20 triplet roots, 10 singlet roots. Orbitals: bonding $\mathrm{d}(\mathrm{xy}) / \mathrm{C}(\pi)$, bonding $\mathrm{d}\left(\mathrm{x}^{2}-\mathrm{y}^{2}\right) / \mathrm{C}\left(\mathrm{sp}^{2}\right), \mathrm{d}(\mathrm{xz}), \mathrm{d}(\mathrm{xy}), \mathrm{d}(\mathrm{yz}), \mathrm{d}\left(\mathrm{z}^{2}\right), \mathrm{d}\left(\mathrm{x}^{2}-\mathrm{y}^{2}\right) / \mathrm{C}\left(\mathrm{sp}^{2}\right)^{*}, \pi^{*}(1)$, $\pi^{*}(2), \pi^{*}(3)$.

\begin{tabular}{|c|c|c|}
\hline CI Vector & Transition & Contribution \\
\hline 2222220000 & Closed shell singlet (CSS) $\mathrm{d}^{8}$ & 0.6318 \\
\hline 2222120100 & $\mathrm{~d}(\mathrm{yz}) \rightarrow \pi^{*}(1)$ & 0.1391 \\
\hline 2122221000 & bonding $\mathrm{d}\left(\mathrm{x}^{2}-\mathrm{y}^{2}\right) / \mathrm{C}\left(\mathrm{sp}^{2}\right) \rightarrow \mathrm{d}\left(\mathrm{x}^{2}-\mathrm{y}^{2}\right) / \mathrm{C}\left(\mathrm{sp}^{2}\right)^{*}$ & 0.0531 \\
\hline 2212220100 & $\mathrm{~d}(\mathrm{xz}) \rightarrow \pi^{*}(1)$ & 0.0283 \\
\hline 2222120001 & $\mathrm{~d}(\mathrm{yz}) \rightarrow \pi^{*}(3)$ & 0.0237 \\
\hline 2022122100 & $2 \mathrm{x}\left[\right.$ bonding $\left.\mathrm{d}\left(\mathrm{x}^{2}-\mathrm{y}^{2}\right) / \mathrm{C}\left(\mathrm{sp}^{2}\right)\right]+\mathrm{d}(\mathrm{yz}) \rightarrow 2 \mathrm{x}\left[\mathrm{d}\left(\mathrm{x}^{2}-\mathrm{y}^{2}\right) / \mathrm{C}\left(\mathrm{sp}^{2}\right)^{*}\right]+\pi^{*}(1)$ & 0.0149 \\
\hline 2122121100 & bonding $\mathrm{d}\left(\mathrm{x}^{2}-\mathrm{y}^{2}\right) / \mathrm{C}\left(\mathrm{sp}^{2}\right)+\mathrm{d}(\mathrm{yz}) \rightarrow \mathrm{d}\left(\mathrm{x}^{2}-\mathrm{y}^{2}\right) / \mathrm{C}\left(\mathrm{sp}^{2}\right)^{*}+\pi^{*}(1)$ & 0.0142 \\
\hline 2212220010 & $\mathrm{~d}(\mathrm{xz}) \rightarrow \pi^{*}(2)$ & 0.0134 \\
\hline 2222202000 & $2 \mathrm{x}\left[\mathrm{d}\left(\mathrm{z}^{2}\right)\right] \rightarrow 2 \mathrm{x}\left[\mathrm{d}\left(\mathrm{x}^{2}-\mathrm{y}^{2}\right) / \mathrm{C}\left(\mathrm{sp}^{2}\right)^{*}\right]$ & 0.0088 \\
\hline 2222210100 & $\mathrm{~d}\left(\mathrm{z}^{2}\right) \rightarrow \pi^{*}(1)$ & 0.0088 \\
\hline 2202222000 & $2 \mathrm{x}[\mathrm{d}(\mathrm{xz})] \rightarrow 2 \mathrm{x}\left[\mathrm{d}\left(\mathrm{x}^{2}-\mathrm{y}^{2}\right) / \mathrm{C}\left(\mathrm{sp}^{2}\right)^{*}\right]$ & 0.0055 \\
\hline 2222022000 & $2 \mathrm{x}[\mathrm{d}(\mathrm{yz})] \rightarrow 2 \mathrm{x}\left[\mathrm{d}\left(\mathrm{x}^{2}-\mathrm{y}^{2}\right) / \mathrm{C}\left(\mathrm{sp}^{2}\right)^{*}\right]$ & 0.0050 \\
\hline 2122121001 & bonding $\mathrm{d}\left(\mathrm{x}^{2}-\mathrm{y}^{2}\right) / \mathrm{C}\left(\mathrm{sp}^{2}\right)+\mathrm{d}(\mathrm{yz})+\rightarrow \mathrm{d}\left(\mathrm{x}^{2}-\mathrm{y}^{2}\right) / \mathrm{C}\left(\mathrm{sp}^{2}\right)^{*}+\pi^{*}(3)$ & 0.0045 \\
\hline 2022122001 & $2 \mathrm{x}\left[\right.$ bonding $\left.\mathrm{d}\left(\mathrm{x}^{2}-\mathrm{y}^{2}\right) / \mathrm{C}\left(\mathrm{sp}^{2}\right)\right]+\mathrm{d}(\mathrm{yz}) \rightarrow 2 \mathrm{x}\left[\mathrm{d}\left(\mathrm{x}^{2}-\mathrm{y}^{2}\right) / \mathrm{C}\left(\mathrm{sp}^{2}\right)^{*}\right]+\pi^{*}(3)$ & 0.0036 \\
\hline 2222120010 & $\mathrm{~d}(\mathrm{yz}) \rightarrow \pi^{*}(2)$ & 0.0031 \\
\hline 2222111100 & $\mathrm{~d}(\mathrm{yz})+\mathrm{d}\left(\mathrm{z}^{2}\right) \rightarrow \mathrm{d}\left(\mathrm{x}^{2}-\mathrm{y}^{2}\right) / \mathrm{C}\left(\mathrm{sp}^{2}\right)^{*}+\pi^{*}(1)$ & 0.0029 \\
\hline 2112221100 & bonding $\mathrm{d}\left(\mathrm{x}^{2}-\mathrm{y}^{2}\right) / \mathrm{C}\left(\mathrm{sp}^{2}\right)+\mathrm{d}(\mathrm{xz}) \rightarrow \mathrm{d}\left(\mathrm{x}^{2}-\mathrm{y}^{2}\right) / \mathrm{C}\left(\mathrm{sp}^{2}\right)^{*}+\pi^{*}(1)$ & 0.0028 \\
\hline 2012222100 & $2 \mathrm{x}\left[\right.$ bonding $\left.\mathrm{d}\left(\mathrm{x}^{2}-\mathrm{y}^{2}\right) / \mathrm{C}\left(\mathrm{sp}^{2}\right)\right]+\mathrm{d}(\mathrm{xz}) \rightarrow 2 \mathrm{x}\left[\mathrm{d}\left(\mathrm{x}^{2}-\mathrm{y}^{2}\right) / \mathrm{C}\left(\mathrm{sp}^{2}\right)^{*}\right]+\pi^{*}(1)$ & 0.0027 \\
\hline \multicolumn{2}{|l|}{ Sum of CSS } & $63 \%$ \\
\hline \multicolumn{2}{|c|}{ Sum of all MLCT } & $22 \%$ \\
\hline \multicolumn{2}{|c|}{ Sum of all d-d } & $7.2 \%$ \\
\hline \multicolumn{2}{|c|}{ Sum of mixed MLCT + d-d } & $4.6 \%$ \\
\hline
\end{tabular}


Table S2F-2. Compound 2 - CASSCF/QD-NEVPT2 composition of the singlet equilibrium ground state in the gas phase. 10o, 12e Active Space - 25 triplet roots, 15 singlet roots. Orbitals: bonding $\mathrm{d}(\mathrm{xy}) / \mathrm{C}(\pi)$, bonding $\mathrm{d}\left(\mathrm{x}^{2}-\mathrm{y}^{2}\right) / \mathrm{C}\left(\mathrm{sp}^{2}\right), \mathrm{d}(\mathrm{xy}), \mathrm{d}(\mathrm{xz}), \mathrm{d}(\mathrm{yz}), \mathrm{d}\left(\mathrm{z}^{2}\right), \mathrm{d}\left(\mathrm{x}^{2}-\mathrm{y}^{2}\right) / \mathrm{C}\left(\mathrm{sp}^{2}\right)^{*}, \pi^{*}(1)$, $\pi^{*}(2), \pi^{*}(3)$.

\begin{tabular}{|c|c|c|}
\hline CI Vector & Transition & Contribution \\
\hline 2222220000 & Closed shell singlet (CSS) $\mathrm{d}^{8}$ & 0.5796 \\
\hline 2222120100 & $\mathrm{~d}(\mathrm{yz}) \rightarrow \pi^{*}(1)$ & 0.1775 \\
\hline 2122221000 & bonding $\mathrm{d}\left(\mathrm{x}^{2}-\mathrm{y}^{2}\right) / \mathrm{C}\left(\mathrm{sp}^{2}\right) \rightarrow \mathrm{d}\left(\mathrm{x}^{2}-\mathrm{y}^{2}\right) / \mathrm{C}\left(\mathrm{sp}^{2}\right)^{*}$ & 0.0644 \\
\hline 2222120010 & $\mathrm{~d}(\mathrm{yz}) \rightarrow \pi^{*}(2)$ & 0.0235 \\
\hline 2221220001 & $\mathrm{~d}(\mathrm{xz}) \rightarrow \pi^{*}(3)$ & 0.0218 \\
\hline 2022122100 & $2 \mathrm{x}\left[\right.$ bonding $\left.\mathrm{d}\left(\mathrm{x}^{2}-\mathrm{y}^{2}\right) / \mathrm{C}\left(\mathrm{sp}^{2}\right)\right]+\mathrm{d}(\mathrm{yz}) \rightarrow 2 \mathrm{x}\left[\mathrm{d}\left(\mathrm{x}^{2}-\mathrm{y}^{2}\right) / \mathrm{C}\left(\mathrm{sp}^{2}\right)^{*}\right]+\pi^{*}(1)$ & 0.0174 \\
\hline 2222210100 & $\mathrm{~d}\left(\mathrm{z}^{2}\right) \rightarrow \pi^{*}(1)$ & 0.0143 \\
\hline 2221220100 & $\mathrm{~d}(\mathrm{xz}) \rightarrow \pi^{*}(1)$ & 0.0121 \\
\hline 2122121100 & bonding $\mathrm{d}\left(\mathrm{x}^{2}-\mathrm{y}^{2}\right) / \mathrm{C}\left(\mathrm{sp}^{2}\right)+\mathrm{d}(\mathrm{yz}) \rightarrow \mathrm{d}\left(\mathrm{x}^{2}-\mathrm{y}^{2}\right) / \mathrm{C}\left(\mathrm{sp}^{2}\right)^{*}+\pi^{*}(1)$ & 0.0121 \\
\hline 2222202000 & $2 \mathrm{x}\left[\mathrm{d}\left(\mathrm{z}^{2}\right)\right] \rightarrow 2 \mathrm{x}\left[\mathrm{d}\left(\mathrm{x}^{2}-\mathrm{y}^{2}\right) / \mathrm{C}\left(\mathrm{sp}^{2}\right)^{*}\right]$ & 0.0083 \\
\hline 2222211000 & $\mathrm{~d}\left(\mathrm{z}^{2}\right) \rightarrow \mathrm{d}\left(\mathrm{x}^{2}-\mathrm{y}^{2}\right) / \mathrm{C}\left(\mathrm{sp}^{2}\right)^{*}$ & 0.0079 \\
\hline 2220222000 & $2 \mathrm{x}[\mathrm{d}(\mathrm{xz})] \rightarrow 2 \mathrm{x}\left[\mathrm{d}\left(\mathrm{x}^{2}-\mathrm{y}^{2}\right) / \mathrm{C}\left(\mathrm{sp}^{2}\right)^{*}\right]$ & 0.0056 \\
\hline 2222022000 & $2 \mathrm{x}[\mathrm{d}(\mathrm{yz})] \rightarrow 2 \mathrm{x}\left[\mathrm{d}\left(\mathrm{x}^{2}-\mathrm{y}^{2}\right) / \mathrm{C}\left(\mathrm{sp}^{2}\right)^{*}\right]$ & 0.0052 \\
\hline 2222111100 & $\mathrm{~d}(\mathrm{yz})+\mathrm{d}\left(\mathrm{z}^{2}\right) \rightarrow \mathrm{d}\left(\mathrm{x}^{2}-\mathrm{y}^{2}\right) / \mathrm{C}\left(\mathrm{sp}^{2}\right)^{*}+\pi^{*}(1)$ & 0.0031 \\
\hline 2212221000 & $\mathrm{~d}(\mathrm{xy}) \rightarrow \mathrm{d}\left(\mathrm{x}^{2}-\mathrm{y}^{2}\right) / \mathrm{C}\left(\mathrm{sp}^{2}\right)^{*}$ & 0.0029 \\
\hline 2022122010 & $2 \mathrm{x}\left[\right.$ bonding $\left.\mathrm{d}\left(\mathrm{x}^{2}-\mathrm{y}^{2}\right) / \mathrm{C}\left(\mathrm{sp}^{2}\right)\right]+\mathrm{d}(\mathrm{yz}) \rightarrow \mathrm{d}\left(\mathrm{x}^{2}-\mathrm{y}^{2}\right) / \mathrm{C}\left(\mathrm{sp}^{2}\right)^{*}+\pi^{*}(2)$ & 0.0025 \\
\hline \multicolumn{2}{|c|}{ Sum of CSS } & $58 \%$ \\
\hline \multicolumn{2}{|c|}{ Sum of all MLCT } & $25 \%$ \\
\hline \multicolumn{2}{|c|}{$\begin{array}{l}\text { Sum of all d-d } \\
\text { Sum of mixed MLCT }+ \text { d-d }\end{array}$} & $\begin{array}{l}9.4 \% \\
3.5 \% \\
\end{array}$ \\
\hline
\end{tabular}


Table S2F-3. Compound 2 - CASSCF/QD-NEVPT2 composition of the singlet equilibrium ground state with CPCM(THF). 10o, 12e Active Space - 25 triplet roots, 15 singlet roots. Orbitals: bonding $\mathrm{d}(\mathrm{xy}) / \mathrm{C}(\pi)$, bonding $\mathrm{d}\left(\mathrm{x}^{2}-\mathrm{y}^{2}\right) / \mathrm{C}\left(\mathrm{sp}^{2}\right), \mathrm{d}(\mathrm{xy}), \mathrm{d}(\mathrm{xz}), \mathrm{d}(\mathrm{yz}), \mathrm{d}\left(\mathrm{z}^{2}\right), \mathrm{d}\left(\mathrm{x}^{2}-\mathrm{y}^{2}\right) / \mathrm{C}\left(\mathrm{sp}^{2}\right)^{*}, \pi^{*}(1)$, $\pi^{*}(2), \pi^{*}(3)$.

\begin{tabular}{llc}
\hline CI Vector & Transition & Contribution \\
\hline 2222220000 & Closed shell singlet $(\mathrm{CSS}) \mathrm{d}^{8}$ & 0.6012 \\
2222120100 & $\mathrm{~d}(\mathrm{yz}) \rightarrow \pi^{*}(1)$ & 0.1574 \\
2122221000 & bonding $\mathrm{d}\left(\mathrm{x}^{2}-\mathrm{y}^{2}\right) / \mathrm{C}\left(\mathrm{sp}^{2}\right) \rightarrow \mathrm{d}\left(\mathrm{x}^{2}-\mathrm{y}^{2}\right) / \mathrm{C}\left(\mathrm{sp}^{2}\right)^{*}$ & 0.0715 \\
2222120010 & $\mathrm{~d}(\mathrm{yz}) \rightarrow \pi^{*}(2)$ & 0.0222 \\
2221220001 & $\mathrm{~d}(\mathrm{xz}) \rightarrow \pi^{*}(3)$ & 0.0195 \\
2022122100 & $\mathrm{~d}(\mathrm{xz}) \rightarrow \pi^{*}(1)$ & 0.0165 \\
2222210100 & $2 \mathrm{x}\left[\mathrm{bonding} \mathrm{d}\left(\mathrm{x}^{2}-\mathrm{y}^{2}\right) / C\left(\mathrm{sp}^{2}\right)\right]+\mathrm{d}(\mathrm{yz}) \rightarrow 2 \mathrm{x}\left[\mathrm{d}\left(\mathrm{x}^{2}-\mathrm{y}^{2}\right) / \mathrm{C}\left(\mathrm{sp}^{2}\right)^{*}\right]+\pi^{*}(1)$ & 0.0157 \\
2221220100 & $\mathrm{bonding} \mathrm{d}\left(\mathrm{x}^{2}-\mathrm{y}^{2}\right) / \mathrm{C}\left(\mathrm{sp}^{2}\right)+\mathrm{d}(\mathrm{yz}) \rightarrow \mathrm{d}\left(\mathrm{x}^{2}-\mathrm{y}^{2}\right) / C\left(\mathrm{sp}^{2}\right)^{*}+\pi^{*}(1)$ & 0.0112 \\
2122121100 & $\mathrm{~d}\left(\mathrm{z}^{2}\right) \rightarrow \pi^{*}(1)$ & 0.0099 \\
2222202000 & $2 \mathrm{x}\left[\mathrm{d}\left(\mathrm{z}^{2}\right)\right] \rightarrow 2 \mathrm{x}\left[\mathrm{d}\left(\mathrm{x}^{2}-\mathrm{y}^{2}\right) / C\left(\mathrm{sp}^{2}\right)^{*}\right]$ & 0.0090 \\
2222211000 & $\mathrm{~d}\left(\mathrm{z}^{2}\right) \rightarrow \mathrm{d}\left(\mathrm{x}^{2}-\mathrm{y}^{2}\right) / \mathrm{C}\left(\mathrm{sp}^{2}\right)^{*}$ & 0.0085 \\
2220222000 & $2 \mathrm{x}[\mathrm{d}(\mathrm{xz})] \rightarrow 2 \mathrm{x}\left[\mathrm{d}\left(\mathrm{x}^{2}-\mathrm{y}^{2}\right) / \mathrm{C}\left(\mathrm{sp}^{2}\right)^{*}\right]$ & 0.0062 \\
2222022000 & $2 \mathrm{x}[\mathrm{d}(\mathrm{yz})] \rightarrow 2 \mathrm{x}\left[\mathrm{d}\left(\mathrm{x}^{2}-\mathrm{y}^{2}\right) / \mathrm{C}\left(\mathrm{sp}^{2}\right)^{*}\right]$ & 0.0057 \\
222211100 & $\mathrm{~d}(\mathrm{yz})+\mathrm{d}\left(\mathrm{z}^{2}\right) \rightarrow \mathrm{d}\left(\mathrm{x}^{2}-\mathrm{y}^{2}\right) / C\left(\mathrm{sp}^{2}\right)^{*}+\pi^{*}(1)$ & 0.0028 \\
\hline Sum of CSS & & $\mathbf{6 0 \%}$ \\
Sum of all MLCT & $\mathbf{2 3 \%}$ \\
Sum of all d-d & & $\mathbf{1 0 \%}$ \\
Sum of mixed MLCT $+\mathbf{d}-\mathbf{d}$ & $\mathbf{3 . 0 \%}$ \\
\hline
\end{tabular}


Table S2F-4. Compound 2 - CASSCF/QD-NEVPT2 composition of the singlet equilibrium ground state in the gas phase. 10o, 12e Active Space - 40 triplet roots, 25 singlet roots. Orbitals: bonding $\mathrm{d}(\mathrm{xy}) / \mathrm{C}(\pi)$, bonding $\mathrm{d}\left(\mathrm{x}^{2}-\mathrm{y}^{2}\right) / \mathrm{C}\left(\mathrm{sp}^{2}\right), \mathrm{d}(\mathrm{xz}), \mathrm{d}(\mathrm{yz}), \mathrm{d}\left(\mathrm{z}^{2}\right), \mathrm{d}(\mathrm{xy}), \mathrm{d}\left(\mathrm{x}^{2}-\mathrm{y}^{2}\right) / \mathrm{C}\left(\mathrm{sp}^{2}\right)^{*}, \pi^{*}(1)$, $\pi^{*}(2), \pi^{*}(3)$.

\begin{tabular}{|c|c|c|}
\hline CI Vector & Transition & Contribution \\
\hline 2222220000 & Closed shell singlet (CSS) $\mathrm{d}^{8}$ & 0.6252 \\
\hline 2221220100 & $\mathrm{~d}(\mathrm{yz}) \rightarrow \pi^{*}(1)$ & 0.0660 \\
\hline 2212220100 & $\mathrm{~d}(\mathrm{xz}) \rightarrow \pi^{*}(1)$ & 0.0637 \\
\hline 2122221000 & bonding $\mathrm{d}\left(\mathrm{x}^{2}-\mathrm{y}^{2}\right) / \mathrm{C}\left(\mathrm{sp}^{2}\right) \rightarrow \mathrm{d}\left(\mathrm{x}^{2}-\mathrm{y}^{2}\right) / \mathrm{C}\left(\mathrm{sp}^{2}\right)^{*}$ & 0.0441 \\
\hline 2212220001 & $\mathrm{~d}(\mathrm{xz}) \rightarrow \pi^{*}(3)$ & 0.0200 \\
\hline 2221220010 & $\mathrm{~d}(\mathrm{yz}) \rightarrow \pi^{*}(2)$ & 0.0188 \\
\hline 2222121000 & $\mathrm{~d}\left(\mathrm{z}^{2}\right) \rightarrow \mathrm{d}\left(\mathrm{x}^{2}-\mathrm{y}^{2}\right) / \mathrm{C}\left(\mathrm{sp}^{2}\right)^{*}$ & 0.0150 \\
\hline 2221220001 & $\mathrm{~d}(\mathrm{yz}) \rightarrow \pi^{*}(3)$ & 0.0127 \\
\hline 2222211000 & $\mathrm{~d}(\mathrm{xy}) \rightarrow \mathrm{d}\left(\mathrm{x}^{2}-\mathrm{y}^{2}\right) / \mathrm{C}\left(\mathrm{sp}^{2}\right)^{*}$ & 0.0104 \\
\hline 2122220100 & bonding $\mathrm{d}\left(\mathrm{x}^{2}-\mathrm{y}^{2}\right) / \mathrm{C}\left(\mathrm{sp}^{2}\right)^{*} \rightarrow \pi^{*}(1)$ & 0.0098 \\
\hline 2212220010 & $\mathrm{~d}(\mathrm{xz}) \rightarrow \pi^{*}(2)$ & 0.0077 \\
\hline 2220222000 & $2 \mathrm{x}[\mathrm{d}(\mathrm{yz})] \rightarrow 2 \mathrm{x}\left[\mathrm{d}\left(\mathrm{x}^{2}-\mathrm{y}^{2}\right) / \mathrm{C}\left(\mathrm{sp}^{2}\right)^{*}\right]$ & 0.0061 \\
\hline 2221121100 & $\mathrm{~d}(\mathrm{yz})+\mathrm{d}\left(\mathrm{z}^{2}\right) \rightarrow \mathrm{d}\left(\mathrm{x}^{2}-\mathrm{y}^{2}\right) / \mathrm{C}\left(\mathrm{sp}^{2}\right)^{*}+\pi^{*}(1)$ & 0.0055 \\
\hline 2202222000 & $2 \mathrm{x}[\mathrm{d}(\mathrm{xz})] \rightarrow 2 \mathrm{x}\left[\mathrm{d}\left(\mathrm{x}^{2}-\mathrm{y}^{2}\right) / \mathrm{C}\left(\mathrm{sp}^{2}\right)^{*}\right]$ & 0.0055 \\
\hline 2222022000 & $2 \mathrm{x}\left[\mathrm{d}\left(\mathrm{z}^{2}\right)\right] \rightarrow 2 \mathrm{x}\left[\mathrm{d}\left(\mathrm{x}^{2}-\mathrm{y}^{2}\right) / \mathrm{C}\left(\mathrm{sp}^{2}\right)^{*}\right]$ & 0.0047 \\
\hline 2122220001 & bonding $\mathrm{d}\left(\mathrm{x}^{2}-\mathrm{y}^{2}\right) / \mathrm{C}\left(\mathrm{sp}^{2}\right) \rightarrow \pi^{*}(3)$ & 0.0046 \\
\hline 2021222100 & $2 \mathrm{x}\left[\right.$ bonding $\left.\mathrm{d}\left(\mathrm{x}^{2}-\mathrm{y}^{2}\right) / \mathrm{C}\left(\mathrm{sp}^{2}\right)\right]+\mathrm{d}(\mathrm{yz}) \rightarrow 2 \mathrm{x}\left[\mathrm{d}\left(\mathrm{x}^{2}-\mathrm{y}^{2}\right) / \mathrm{C}\left(\mathrm{sp}^{2}\right)^{*}\right]+\pi^{*}(1)$ & 0.0045 \\
\hline 2212221000 & $\mathrm{~d}(\mathrm{xz}) \rightarrow \mathrm{d}\left(\mathrm{x}^{2}-\mathrm{y}^{2}\right) / \mathrm{C}\left(\mathrm{sp}^{2}\right)^{*}$ & 0.0041 \\
\hline 2012222100 & $2 \mathrm{x}\left[\right.$ bonding $\left.\mathrm{d}\left(\mathrm{x}^{2}-\mathrm{y}^{2}\right) / \mathrm{C}\left(\mathrm{sp}^{2}\right)\right]+\mathrm{d}(\mathrm{xz}) \rightarrow 2 \mathrm{x}\left[\mathrm{d}\left(\mathrm{x}^{2}-\mathrm{y}^{2}\right) / \mathrm{C}\left(\mathrm{sp}^{2}\right)^{*}\right]+\pi^{*}(1)$ & 0.0036 \\
\hline 2121221100 & bonding $\mathrm{d}\left(\mathrm{x}^{2}-\mathrm{y}^{2}\right) / \mathrm{C}\left(\mathrm{sp}^{2}\right)+\mathrm{d}(\mathrm{yz}) \rightarrow \mathrm{d}\left(\mathrm{x}^{2}-\mathrm{y}^{2}\right) / \mathrm{C}\left(\mathrm{sp}^{2}\right)^{*}+\pi^{*}(1)$ & 0.0034 \\
\hline 2222120100 & $\mathrm{~d}\left(\mathrm{z}^{2}\right) \rightarrow \pi^{*}(1)$ & 0.0032 \\
\hline 2112221100 & bonding $\mathrm{d}\left(\mathrm{x}^{2}-\mathrm{y}^{2}\right) / \mathrm{C}\left(\mathrm{sp}^{2}\right)+\mathrm{d}(\mathrm{xz}) \rightarrow \mathrm{d}\left(\mathrm{x}^{2}-\mathrm{y}^{2}\right) / \mathrm{C}\left(\mathrm{sp}^{2}\right)^{*}+\pi^{*}(1)$ & 0.0030 \\
\hline 2222210100 & $\mathrm{~d}(\mathrm{xy}) \rightarrow \pi^{*}(1)$ & 0.0026 \\
\hline 1222221000 & bonding $\mathrm{d}(\mathrm{xy}) / \mathrm{C}(\pi) \rightarrow \mathrm{d}\left(\mathrm{x}^{2}-\mathrm{y}^{2}\right) / \mathrm{C}\left(\mathrm{sp}^{2}\right)^{*}$ & 0.0026 \\
\hline \multicolumn{2}{|c|}{ Sum of CSS } & $63 \%$ \\
\hline \multicolumn{2}{|c|}{ Sum of all MLCT } & $21 \%$ \\
\hline \multicolumn{2}{|c|}{$\begin{array}{l}\text { Sum of all d-d } \\
\text { Sum of mixed MLCT }+ \text { d-d }\end{array}$} & $\begin{array}{l}9.3 \% \\
2.0 \%\end{array}$ \\
\hline
\end{tabular}


Table S2G. Compound 2 -CASSCF/QD-NEVPT2 composition of the singlet equilibrium ground state while varying number roots. 9o, 10e Active Space -25 triplet roots, $X$ singlet roots (where $X$ ranged from 15 to 25). Orbitals: bonding $\mathrm{d}\left(\mathrm{x}^{2}-\mathrm{y}^{2}\right) / \mathrm{C}\left(\mathrm{sp}^{2}\right), \mathrm{d}(\mathrm{xy}), \mathrm{d}(\mathrm{xz}), \mathrm{d}(\mathrm{yz}), \mathrm{d}\left(\mathrm{z}^{2}\right)$, $\mathrm{d}\left(\mathrm{x}^{2}-\mathrm{y}^{2}\right) / \mathrm{C}\left(\mathrm{sp}^{2}\right)^{*}, \pi^{*}(1), \pi^{*}(2), \pi^{*}(3) .10 \mathrm{o}, 12 \mathrm{e}$ Active Space $-X$ triplet roots, $Y$ singlet roots where $X$ ranged from 20 to 40 , and $Y$ ranged from 10 to 25 . Orbitals: bonding $\mathrm{d}(\mathrm{xy}) / \mathrm{C}(\pi)$, bonding $\mathrm{d}\left(\mathrm{x}^{2}-\mathrm{y}^{2}\right) / \mathrm{C}\left(\mathrm{sp}^{2}\right), \mathrm{d}(\mathrm{xy}), \mathrm{d}(\mathrm{xz}), \mathrm{d}(\mathrm{yz}), \mathrm{d}\left(\mathrm{z}^{2}\right), \mathrm{d}\left(\mathrm{x}^{2}-\mathrm{y}^{2}\right) / \mathrm{C}\left(\mathrm{sp}^{2}\right)^{*}, \pi^{*}(1), \pi^{*}(2), \pi^{*}(3)$.

\begin{tabular}{|c|c|c|c|c|c|c|c|c|}
\hline \multicolumn{9}{|c|}{ Weights of Three Most Dominant Terms in CI-Vector (\# Triplets / \# Singlets) } \\
\hline Active Spaces: & \multicolumn{4}{|c|}{9 orbitals, 10 electrons } & \multicolumn{4}{|c|}{10 orbitals, 12 electrons } \\
\hline Configuration & $\begin{array}{c}25 / 15 \\
\text { Gas }\end{array}$ & $\begin{array}{c}25 / 15 \\
\text { CРCM }\end{array}$ & $\begin{array}{c}25 / 25 \\
\text { Gas }\end{array}$ & $\begin{array}{c}25 / 25 \\
\text { CPCM }\end{array}$ & $\begin{array}{c}\text { 20/10 } \\
\text { Gas }\end{array}$ & $\begin{array}{c}25 / 15 \\
\text { Gas }\end{array}$ & $\begin{array}{c}25 / 15 \\
\text { CPCM }\end{array}$ & $\begin{array}{c}40 / 25 \\
\text { Gas }\end{array}$ \\
\hline CSS & 0.5509 & 0.5730 & 0.552 & 0.5464 & 0.6318 & 0.5796 & 0.6012 & 0.6252 \\
\hline $\mathrm{d}(\mathrm{yz}) \rightarrow \pi^{*}(1)$ & 0.1282 & 0.1468 & 0.088 & 0.1039 & 0.1391 & 0.1775 & 0.1574 & $0.0660^{\dagger}$ \\
\hline $\begin{array}{l}\mathrm{d}\left(\mathrm{x}^{2}-\mathrm{y}^{2}\right) / \mathrm{C}\left(\mathrm{sp}^{2}\right) \rightarrow \\
\mathrm{d}\left(\mathrm{x}^{2}-\mathrm{y}^{2}\right) / \mathrm{C}\left(\mathrm{sp}^{2}\right)^{*}\end{array}$ & 0.0888 & 0.0958 & 0.135 & $0.0853^{\ddagger}$ & 0.0531 & 0.0644 & 0.0715 & 0.0441 \\
\hline \multicolumn{9}{|c|}{ Sums of Weights } \\
\hline Active Spaces: & \multicolumn{4}{|c|}{9 orbitals, 10 electrons } & \multicolumn{4}{|c|}{10 orbitals, 12 electrons } \\
\hline Configuration & $\begin{array}{c}25 / 15 \\
\text { Gas }\end{array}$ & $\begin{array}{c}25 / 15 \\
\text { CРCM }\end{array}$ & $\begin{array}{c}25 / 25 \\
\text { Gas }\end{array}$ & $\begin{array}{c}25 / 25 \\
\text { CPCM }\end{array}$ & $\begin{array}{c}\text { 20/10 } \\
\text { Gas }\end{array}$ & $\begin{array}{c}25 / 15 \\
\text { Gas }\end{array}$ & $\begin{array}{c}25 / 15 \\
\text { CPCM }\end{array}$ & $\begin{array}{c}40 / 25 \\
\text { Gas }\end{array}$ \\
\hline CSS & $55 \%$ & $57 \%$ & $55 \%$ & $55 \%$ & $63 \%$ & $58 \%$ & $60 \%$ & $63 \%$ \\
\hline Sum of MLCT & $26 \%$ & $23 \%$ & $18 \%$ & $20 \%$ & $22 \%$ & $25 \%$ & $23 \%$ & $21 \%$ \\
\hline Sum of d-d & $12 \%$ & $13 \%$ & $21 \%$ & $20 \%$ & $7.2 \%$ & $9.4 \%$ & $10 \%$ & $9.3 \%$ \\
\hline Sum of MLCT $+d-d$ & $3.1 \%$ & $2.8 \%$ & $2.3 \%$ & $1.5 \%$ & $4.6 \%$ & $3.5 \%$ & $3.0 \%$ & $2.0 \%$ \\
\hline \multicolumn{9}{|c|}{ Singlet $\rightarrow$ Singlet Transition Energies $\left(\mathrm{kcal} \mathrm{mol}^{-1}\right)$} \\
\hline Active Spaces: & \multicolumn{4}{|c|}{9 orbitals, 10 electrons } & \multicolumn{4}{|c|}{10 orbitals, 12 electrons } \\
\hline Transition & $\begin{array}{l}25 / 15 \\
\text { Gas }\end{array}$ & $\begin{array}{c}25 / 15 \\
\text { CРCM }\end{array}$ & $\begin{array}{l}\text { 25/25 } \\
\text { Gas }\end{array}$ & 25/25 СРCM & $\begin{array}{c}20 / 10 \\
\text { Gas }\end{array}$ & $\begin{array}{c}\text { 25/15 } \\
\text { Gas }\end{array}$ & $\begin{array}{c}25 / 15 \\
\text { CPCM }\end{array}$ & $\begin{array}{c}40 / 25 \\
\text { Gas }\end{array}$ \\
\hline $\mathrm{S}_{0} \rightarrow \mathrm{S}_{1}$ & 33.3 & 37.2 & 39.1 & 39.2 & 29.8 & 32.1 & 35.5 & 37.1 \\
\hline $\mathrm{S}_{0} \rightarrow \mathrm{S}_{2}$ & 40.6 & 44.3 & 45.1 & 45.8 & 36.9 & 39.7 & 42.9 & 43.3 \\
\hline $\mathrm{S}_{0} \rightarrow \mathrm{S}_{3}$ & 45.5 & 49.5 & 50.6 & 50.2 & 46.2 & 45.2 & 48.9 & 47.8 \\
\hline $\mathrm{S}_{0} \rightarrow \mathrm{S}_{4}$ & 58.1 & 62.0 & 63.8 & 64.3 & 51.0 & 55.2 & 58.6 & 60.1 \\
\hline $\mathrm{S}_{0} \rightarrow \mathrm{S}_{5}$ & 59.3 & 63.7 & 65.5 & 66.1 & 65.9 & 58.6 & 62.7 & 62.9 \\
\hline $\mathrm{S}_{0} \rightarrow \mathrm{S}_{6}$ & 63.7 & 65.0 & 67.8 & 69.7 & 73.1 & 62.3 & 64.5 & 66.8 \\
\hline $\mathrm{S}_{0} \rightarrow \mathrm{S}_{7}$ & 66.3 & 69.1 & 71.7 & 70.7 & 76.5 & 64.6 & 69.7 & 70.2 \\
\hline $\mathrm{S}_{0} \rightarrow \mathrm{S}_{8}$ & 68.2 & 72.0 & 71.9 & 71.5 & 78.4 & 66.5 & 71.7 & 71.5 \\
\hline $\mathrm{S}_{0} \rightarrow \mathrm{S}_{9}$ & 69.2 & 72.8 & 73.4 & 72.1 & 81.4 & 71.4 & 73.7 & 72.3 \\
\hline $\mathrm{S}_{0} \rightarrow \mathrm{S}_{10}$ & 73.0 & 74.1 & 73.5 & 74.3 & --- & 75.5 & 74.7 & 72.7 \\
\hline $\mathrm{S}_{0} \rightarrow \mathrm{S}_{11}$ & 74.6 & 75.3 & 75.2 & 74.8 & --- & 76.9 & 76.5 & 74.2 \\
\hline $\mathrm{S}_{0} \rightarrow \mathrm{S}_{12}$ & 75.9 & 77.7 & 77.2 & 75.7 & --- & 78.7 & 78.7 & 76.9 \\
\hline $\mathrm{S}_{0} \rightarrow \mathrm{S}_{13}$ & 79.0 & 80.4 & 79.8 & 77.5 & --- & 80.2 & 81.2 & 77.2 \\
\hline $\mathrm{S}_{0} \rightarrow \mathrm{S}_{14}$ & 82.6 & 86.3 & 84.2 & 87.1 & --- & 112.7 & 116.2 & 84.2 \\
\hline
\end{tabular}

${ }^{\dagger} \mathrm{d}(\mathrm{xz}) \rightarrow \pi^{*}(1)$ is $0.0637 .{ }^{*} \mathrm{~d}\left(\mathrm{z}^{2}\right) \rightarrow \mathrm{d}\left(\mathrm{x}^{2}-\mathrm{y}^{2}\right)$ is slightly higher at 0.0858 . 
Table S2H-1. Compound 2 with Ni-C distance of $3.14 \AA-$ CASSCF/QD-NEVPT2 lowest transition energies in the gas phase. 8o, 10e Active Space - 25 triplet roots, 15 singlet roots. Orbitals (in order for CI vector notation below): $\mathrm{d}(\mathrm{xz}), \mathrm{d}(\mathrm{yz}), \mathrm{d}(\mathrm{xy}), \mathrm{d}\left(\mathrm{z}^{2}\right), \mathrm{d}\left(\mathrm{x}^{2}-\mathrm{y}^{2}\right), \mathrm{C}\left(\mathrm{sp}^{2}\right)^{*}, \pi^{*}(1)$, $\pi^{*}(2)$.

\begin{tabular}{|c|c|c|c|c|c|c|c|}
\hline State & Root & Multiplicity & $\Delta \mathrm{E} / \mathrm{nm}$ & $\Delta \mathrm{E} / \mathrm{kcal} \mathrm{mol}^{-1}$ & CI Vector & $f_{\text {osc }}$ & Contribution \\
\hline \multirow[t]{3}{*}{0} & 0 & $3^{\dagger}$ & --- & --- & 12221110 & --- & 0.4414 \\
\hline & & & & & 22211110 & --- & 0.0777 \\
\hline & & & & & 21221110 & --- & 0.0769 \\
\hline \multirow[t]{2}{*}{1} & 0 & $1^{\dagger}$ & $1.35 \mathrm{E}+05$ & 0.2 & 12221110 & --- & 0.4544 \\
\hline & & & & & 22220110 & --- & 0.1621 \\
\hline \multirow[t]{2}{*}{2} & 1 & 3 & 11691.9 & 2.4 & 22211110 & 0.0000185 & 0.5698 \\
\hline & & & & & 22211200 & --- & 0.1445 \\
\hline \multirow[t]{2}{*}{3} & 1 & 1 & 6953.1 & 4.1 & 22211110 & --- & 0.7473 \\
\hline & & & & & 21221110 & --- & 0.0745 \\
\hline \multirow[t]{2}{*}{4} & 2 & 3 & 6042.1 & 4.7 & 22211110 & 0.0000044 & 0.5075 \\
\hline & & & & & 21221110 & --- & 0.1900 \\
\hline \multirow[t]{2}{*}{5} & 3 & 3 & 5510.1 & 5.2 & 22211110 & 0.0000638 & 0.3270 \\
\hline & & & & & 21221110 & --- & 0.3178 \\
\hline \multirow[t]{2}{*}{6} & 2 & 1 & 4415.9 & 6.5 & 22220110 & --- & 0.4317 \\
\hline & & & & & 12221110 & --- & 0.2298 \\
\hline \multirow[t]{2}{*}{7} & 4 & 3 & 3990.5 & 7.2 & 12221110 & 0.0000214 & 0.4491 \\
\hline & & & & & 22220110 & --- & 0.1777 \\
\hline \multirow[t]{2}{*}{8} & 5 & 3 & 3896.3 & 7.3 & 21221110 & 0.0000363 & 0.6461 \\
\hline & & & & & 22220110 & --- & 0.1142 \\
\hline \multirow[t]{2}{*}{9} & 3 & 1 & 3858.7 & 7.4 & 21221110 & --- & 0.7457 \\
\hline & & & & & 12221110 & --- & 0.0583 \\
\hline \multirow[t]{2}{*}{10} & 6 & 3 & 3537.3 & 8.1 & 22220110 & 0.0000600 & 0.4848 \\
\hline & & & & & 12221110 & --- & 0.2458 \\
\hline \multirow[t]{2}{*}{11} & 7 & 3 & 1142.4 & 25.0 & 22121110 & 0.0000216 & 0.4116 \\
\hline & & & & & 22121200 & --- & 0.1173 \\
\hline \multirow[t]{2}{*}{12} & 4 & 1 & 1063.8 & 26.9 & 22121110 & --- & 0.5493 \\
\hline & & & & & 11222110 & --- & 0.2679 \\
\hline \multirow[t]{2}{*}{13} & 8 & 3 & 1042.4 & 27.4 & 22121110 & 0.0000031 & 0.5686 \\
\hline & & & & & 11222110 & --- & 0.2627 \\
\hline \multirow[t]{2}{*}{14} & 9 & 3 & 966.1 & 29.6 & 21221101 & 0.0536947 & 0.4585 \\
\hline & & & & & 12221101 & --- & 0.1746 \\
\hline \multirow[t]{2}{*}{15} & 5 & 1 & 953.6 & 30.0 & 22211101 & --- & 0.4312 \\
\hline & & & & & 21221101 & --- & 0.2753 \\
\hline \multirow[t]{2}{*}{16} & 10 & 3 & 947.7 & 30.2 & 22211101 & 0.0008840 & 0.7957 \\
\hline & & & & & 22211011 & --- & 0.0538 \\
\hline \multirow[t]{2}{*}{17} & 6 & 1 & 916.4 & 31.2 & 22211101 & --- & 0.3968 \\
\hline & & & & & 21221101 & --- & 0.2779 \\
\hline \multirow[t]{2}{*}{18} & 11 & 3 & 887.2 & 32.2 & 22211101 & 0.0003050 & 0.7210 \\
\hline & & & & & 21221101 & --- & 0.1448 \\
\hline \multirow[t]{2}{*}{19} & 12 & 3 & 847.2 & 33.7 & 21221101 & 0.0000199 & 0.8183 \\
\hline & & & & & 21221011 & --- & 0.0548 \\
\hline \multirow[t]{2}{*}{20} & 13 & 3 & 825.5 & 34.6 & 12221101 & 0.0035754 & 0.7783 \\
\hline & & & & & 22121101 & --- & 0.0461 \\
\hline
\end{tabular}

†The lowest singlet and triplet roots are essentially isoenergetic. 
Table S2H-2. Compound 2 with Ni-C distance of $3.14 \AA$ - CASSCF/QD-NEVPT2 lowest transition energies with $\mathrm{CPCM}(\mathrm{THF}) .80,10 \mathrm{e}$ Active Space -25 triplet roots, 15 singlet roots. Orbitals (in order for CI vector notation below): $\mathrm{d}(\mathrm{xy}), \mathrm{d}(\mathrm{xz}), \mathrm{d}\left(\mathrm{z}^{2}\right), \mathrm{d}(\mathrm{yz}), \mathrm{d}\left(\mathrm{x}^{2}-\mathrm{y}^{2}\right), \mathrm{C}\left(\mathrm{sp}^{2}\right)^{*}, \pi^{*}(1)$, $\pi^{*}(2)$.

\begin{tabular}{|c|c|c|c|c|c|c|c|}
\hline State & Root & Multiplicity & $\Delta \mathrm{E} / \mathbf{n m}$ & $\Delta \mathrm{E} / \mathrm{kcal} \mathrm{mol}^{-1}$ & CI Vector & $f_{\text {osc }}$ & Contribution \\
\hline \multirow[t]{3}{*}{0} & 0 & $3^{\dagger}$ & --- & --- & 21221110 & --- & 0.4372 \\
\hline & & & & & 22121110 & --- & 0.1725 \\
\hline & & & & & 22211110 & --- & 0.0831 \\
\hline \multirow[t]{2}{*}{1} & 0 & $1^{\dagger}$ & $5.9 \mathrm{E}+04$ & 0.5 & 21221110 & --- & 0.4877 \\
\hline & & & & & 22220110 & --- & 0.1626 \\
\hline \multirow[t]{2}{*}{2} & 1 & 3 & 13662.2 & 2.1 & 22121110 & 0.0000327 & 0.5328 \\
\hline & & & & & 22211110 & --- & 0.1036 \\
\hline \multirow[t]{2}{*}{3} & 1 & 1 & 7957.3 & 3.6 & 22121110 & --- & 0.8617 \\
\hline & & & & & 21221110 & --- & 0.0331 \\
\hline \multirow[t]{2}{*}{4} & 2 & 3 & 6570.1 & 4.4 & 22121110 & 0.0000085 & 0.6242 \\
\hline & & & & & 22211110 & --- & 0.1966 \\
\hline \multirow[t]{2}{*}{5} & 3 & 3 & 5735.6 & 5.0 & 22211110 & 0.0000302 & 0.3595 \\
\hline & & & & & 22121110 & --- & 0.2485 \\
\hline \multirow[t]{2}{*}{6} & 2 & 1 & 4184.7 & 6.8 & 22220110 & --- & 0.5189 \\
\hline & & & & & 21221110 & --- & 0.1758 \\
\hline \multirow[t]{2}{*}{7} & 4 & 3 & 4034.3 & 7.1 & 21221110 & 0.0000046 & 0.6509 \\
\hline & & & & & 22220110 & --- & 0.0728 \\
\hline \multirow[t]{2}{*}{8} & 5 & 3 & 3982.8 & 7.2 & 22211110 & 0.0000565 & 0.7504 \\
\hline & & & & & 21221110 & --- & 0.0852 \\
\hline \multirow[t]{2}{*}{9} & 3 & 1 & 3963.4 & 7.2 & 22211110 & --- & 0.7430 \\
\hline & & & & & 21221110 & --- & 0.1371 \\
\hline \multirow[t]{2}{*}{10} & 6 & 3 & 3416.6 & 8.4 & 22220110 & 0.0000134 & 0.7020 \\
\hline & & & & & 21221110 & --- & 0.1408 \\
\hline \multirow[t]{2}{*}{11} & 7 & 3 & 1144.3 & 25.0 & 12221110 & 0.0000176 & 0.5107 \\
\hline & & & & & 21212110 & --- & 0.2185 \\
\hline \multirow[t]{2}{*}{12} & 4 & 1 & 1068.5 & 26.8 & 12221110 & --- & 0.6071 \\
\hline & & & & & 21212110 & --- & 0.2776 \\
\hline \multirow[t]{2}{*}{13} & 8 & 3 & 1043.9 & 27.4 & 12221110 & 0.0000045 & 0.6244 \\
\hline & & & & & 21212110 & --- & 0.2658 \\
\hline \multirow[t]{2}{*}{14} & 5 & 1 & 1007.8 & 28.4 & 22121101 & --- & 0.7969 \\
\hline & & & & & 21221101 & --- & 0.0324 \\
\hline \multirow[t]{2}{*}{15} & 9 & 3 & 989.2 & 28.9 & 22121101 & 0.0042687 & 0.6679 \\
\hline & & & & & 22211101 & --- & 0.1223 \\
\hline \multirow[t]{2}{*}{16} & 10 & 3 & 975.5 & 29.3 & 22121101 & 0.0457152 & 0.4067 \\
\hline & & & & & 22211101 & --- & 0.3207 \\
\hline \multirow[t]{2}{*}{17} & 6 & 1 & 953.3 & 30.0 & 22211101 & --- & 0.6362 \\
\hline & & & & & 21221101 & --- & 0.1400 \\
\hline \multirow[t]{2}{*}{18} & 11 & 3 & 925.9 & 30.9 & 22121101 & 0.0001487 & 0.6010 \\
\hline & & & & & 22211101 & --- & 0.3172 \\
\hline \multirow[t]{2}{*}{19} & 12 & 3 & 883.5 & 32.4 & 22211101 & 0.0000838 & 0.8077 \\
\hline & & & & & 21122101 & --- & 0.0425 \\
\hline \multirow[t]{2}{*}{20} & 7 & 1 & 862.1 & 33.2 & 21221101 & --- & 0.6712 \\
\hline & & & & & 22211101 & & 0.1758 \\
\hline
\end{tabular}

${ }^{\dagger}$ The lowest singlet and triplet roots are essentially isoenergetic. 
Table S2I-1. Compound 2 - CASSCF/QD-NEVPT2 composition with Ni-C distance of $3.14 \AA$ CASSCF/QD-NEVPT2 composition of the lowest singlet root in the gas phase. 8o, 10e Active Space -25 triplet roots, 15 singlet roots. Orbitals (in order for CI vector notation below): $d(x z)$, $\mathrm{d}(\mathrm{yz}), \mathrm{d}(\mathrm{xy}), \mathrm{d}\left(\mathrm{z}^{2}\right), \mathrm{d}\left(\mathrm{x}^{2}-\mathrm{y}^{2}\right), \mathrm{C}\left(\mathrm{sp}^{2}\right)^{*}, \pi^{*}(1), \pi^{*}(2)$.

\begin{tabular}{llc}
\hline CI Vector & Transition & Contribution \\
\hline 12221110 & $\mathrm{~d}(\mathrm{xz})+\mathrm{d}\left(\mathrm{x}^{2}-\mathrm{y}^{2}\right) \rightarrow \mathrm{C}\left(\mathrm{sp}^{2}\right)^{*}+\pi^{*}(1)$ & 0.4544 \\
22220110 & $2 \mathrm{x}\left[\mathrm{d}\left(\mathrm{x}^{2}-\mathrm{y}^{2}\right)\right] \rightarrow \mathrm{C}\left(\mathrm{sp}^{2}\right)^{*}+\pi^{*}(1)$ & 0.1621 \\
22211110 & $\mathrm{~d}\left(\mathrm{z}^{2}\right)+\mathrm{d}\left(\mathrm{x}^{2}-\mathrm{y}^{2}\right) \rightarrow \mathrm{C}\left(\mathrm{sp}^{2}\right)^{*}+\pi^{*}(1)$ & 0.0698 \\
22220200 & $2 \mathrm{x}\left[\mathrm{d}\left(\mathrm{x}^{2}-\mathrm{y}^{2}\right)\right] \rightarrow 2 \mathrm{x}\left[\mathrm{C}\left(\mathrm{sp}^{2}\right)^{*}\right]$ & 0.0422 \\
21221110 & $\mathrm{~d}(\mathrm{yz})+\mathrm{d}\left(\mathrm{x}^{2}-\mathrm{y}^{2}\right) \rightarrow \mathrm{C}\left(\mathrm{sp}^{2}\right)^{*}+\pi^{*}(1)$ & 0.0279 \\
22220020 & $2 \mathrm{x}\left[\mathrm{d}\left(\mathrm{x}^{2}-\mathrm{y}^{2}\right)\right] \rightarrow 2 \mathrm{x}\left[\pi^{*}(1)\right]$ & 0.0270 \\
12222010 & $\mathrm{~d}(\mathrm{xz}) \rightarrow \pi^{*}(1)$ & 0.0157 \\
21122110 & $\mathrm{~d}(\mathrm{yz})+\mathrm{d}(\mathrm{xy}) \rightarrow \mathrm{C}\left(\mathrm{sp}^{2}\right)^{*}+\pi^{*}(1)$ & 0.0151 \\
21221101 & $\mathrm{~d}(\mathrm{yz})+\mathrm{d}\left(\mathrm{x}^{2}-\mathrm{y}^{2}\right) \rightarrow \mathrm{C}\left(\mathrm{sp}^{2}\right)^{*}+\pi^{*}(2)$ & 0.0149 \\
21212110 & $\mathrm{~d}(\mathrm{yz})+\mathrm{d}\left(\mathrm{z}^{2}\right) \rightarrow \mathrm{C}\left(\mathrm{sp}^{2}\right)^{*}+\pi^{*}(1)$ & 0.0144 \\
22121110 & $\mathrm{~d}(\mathrm{xy})+\mathrm{d}\left(\mathrm{x}^{2}-\mathrm{y}^{2}\right) \rightarrow \mathrm{C}\left(\mathrm{sp}^{2}\right)^{*}+\pi^{*}(2)$ & 0.0105 \\
12220210 & $\mathrm{~d}(\mathrm{xz})+2 \mathrm{x}\left[\mathrm{d}\left(\mathrm{x}^{2}-\mathrm{y}^{2}\right)\right] \rightarrow 2 \mathrm{x}\left[\mathrm{C}\left(\mathrm{sp}^{2}\right)^{*}\right]+\pi^{*}(1)$ & 0.0092 \\
12222100 & $\mathrm{~d}(\mathrm{xz}) \rightarrow \mathrm{C}\left(\mathrm{sp}^{2}\right)^{*}$ & 0.0067 \\
12221101 & $\mathrm{~d}(\mathrm{xz})+\mathrm{d}\left(\mathrm{x}^{2}-\mathrm{y}^{2}\right) \rightarrow \mathrm{C}\left(\mathrm{sp}^{2}\right)^{*}+\pi^{*}(2)$ & 0.0065 \\
22202110 & $2 \mathrm{x}\left[\mathrm{d}\left(\mathrm{z}^{2}\right)\right] \rightarrow \mathrm{C}\left(\mathrm{sp}^{2}\right)^{*}+\pi^{*}(1)$ & 0.0056 \\
20222110 & $2 \mathrm{x}[\mathrm{d}(\mathrm{yz})] \rightarrow \mathrm{C}\left(\mathrm{sp}^{2}\right)^{*}+\pi^{*}(1)$ & 0.0039 \\
02222110 & $2 \mathrm{x}[\mathrm{d}(\mathrm{xz})] \rightarrow \mathrm{C}\left(\mathrm{sp}^{2}\right)^{*}+\pi^{*}(1)$ & 0.0038 \\
\hline
\end{tabular}

Table S2I-2. Compound 2 - CASSCF/QD-NEVPT2 composition with Ni-C distance of $3.14 \AA$ CASSCF/QD-NEVPT2 composition of the lowest singlet root with CPCM(THF). 8o, 10e Active Space - 25 triplet roots, 15 singlet roots. Orbitals (in order for CI vector notation below): $d(x y)$, $\mathrm{d}(\mathrm{xz}), \mathrm{d}\left(\mathrm{z}^{2}\right), \mathrm{d}(\mathrm{yz}), \mathrm{d}\left(\mathrm{x}^{2}-\mathrm{y}^{2}\right), \mathrm{C}\left(\mathrm{sp}^{2}\right)^{*}, \pi^{*}(1), \pi^{*}(2)$.

\begin{tabular}{llc}
\hline CI Vector & Transition & Contribution \\
\hline 21221110 & $\mathrm{~d}(\mathrm{yz})+\mathrm{d}\left(\mathrm{x}^{2}-\mathrm{y}^{2}\right) \rightarrow \mathrm{C}\left(\mathrm{sp}^{2}\right)^{*}+\pi^{*}(1)$ & 0.4877 \\
22220110 & $2 \mathrm{x}\left[\mathrm{d}\left(\mathrm{x}^{2}-\mathrm{y}^{2}\right)\right] \rightarrow \mathrm{C}\left(\mathrm{sp}^{2}\right)^{*}+\pi^{*}(1)$ & 0.1626 \\
22211110 & $\mathrm{~d}\left(\mathrm{z}^{2}\right)+\mathrm{d}\left(\mathrm{x}^{2}-\mathrm{y}^{2}\right) \rightarrow \mathrm{C}\left(\mathrm{sp}^{2}\right)^{*}+\pi^{*}(1)$ & 0.0842 \\
22221100 & $\mathrm{~d}\left(\mathrm{x}^{2}-\mathrm{y}^{2}\right) \rightarrow \mathrm{C}\left(\mathrm{sp}^{2}\right)^{*}$ & 0.0671 \\
22121110 & $\mathrm{~d}(\mathrm{xy})+\mathrm{d}\left(\mathrm{x}^{2}-\mathrm{y}^{2}\right) \rightarrow \mathrm{C}\left(\mathrm{sp}^{2}\right)^{*}+\pi^{*}(1)$ & 0.0307 \\
22220200 & $2 \mathrm{x}\left[\mathrm{d}\left(\mathrm{x}^{2}-\mathrm{y}^{2}\right)\right] \rightarrow 2 \mathrm{x}\left[\mathrm{C}\left(\mathrm{sp}^{2}\right)^{*}\right]$ & 0.0273 \\
22112110 & $\mathrm{~d}(\mathrm{xy})+\mathrm{d}\left(\mathrm{z}^{2}\right) \rightarrow \mathrm{C}\left(\mathrm{sp}^{2}\right)^{*}+\pi^{*}(1)$ & 0.0189 \\
22220020 & $2 \mathrm{x}\left[\mathrm{d}\left(\mathrm{x}^{2}-\mathrm{y}^{2}\right)\right] \rightarrow 2 \mathrm{x}\left[\pi^{*}(1)\right]$ & 0.0149 \\
12212110 & $\mathrm{~d}(\mathrm{xz})+\mathrm{d}\left(\mathrm{z}^{2}\right) \rightarrow \mathrm{C}\left(\mathrm{sp}^{2}\right)^{*}+\pi^{*}(1)$ & 0.0136 \\
21222010 & $\mathrm{~d}(\mathrm{yz}) \rightarrow \pi^{*}(1)$ & 0.0123 \\
22211101 & $\mathrm{~d}\left(\mathrm{z}^{2}\right)+\mathrm{d}\left(\mathrm{x}^{2}-\mathrm{y}^{2}\right) \rightarrow \mathrm{C}\left(\mathrm{sp}^{2}\right)^{*}+\pi^{*}(2)$ & 0.0102 \\
12221110 & $\mathrm{~d}(\mathrm{xz})+\mathrm{d}\left(\mathrm{x}^{2}-\mathrm{y}^{2}\right) \rightarrow \mathrm{C}\left(\mathrm{sp}^{2}\right)^{*}+\pi^{*}(1)$ & 0.0087 \\
21220210 & $\mathrm{~d}(\mathrm{yz})+2 \mathrm{x}\left[\mathrm{d}\left(\mathrm{x}^{2}-\mathrm{y}^{2}\right)\right] \rightarrow 2 \mathrm{x}\left[\mathrm{C}\left(\mathrm{sp}^{2}\right)^{*}\right]+\pi^{*}(1)$ & 0.0071 \\
22022110 & $2 \mathrm{x}[\mathrm{d}(\mathrm{xy})] \rightarrow \mathrm{C}\left(\mathrm{sp}^{2}\right)^{*}+\pi^{*}(1)$ & 0.0064 \\
21222100 & $\mathrm{~d}(\mathrm{xz}) \rightarrow \mathrm{C}\left(\mathrm{sp}^{2}\right)^{*}$ & 0.0060 \\
22121101 & $\mathrm{~d}(\mathrm{xy})+\mathrm{d}\left(\mathrm{x}^{2}-\mathrm{y}^{2}\right) \rightarrow \mathrm{C}\left(\mathrm{sp}^{2}\right)^{*}+\pi^{*}(2)$ & 0.0053 \\
20222110 & $2 \mathrm{x}[\mathrm{d}(\mathrm{yz})] \rightarrow \mathrm{C}\left(\mathrm{sp}^{2}\right)^{*}+\pi^{*}(1)$ & 0.0044 \\
22202110 & $2 \mathrm{x}\left[\mathrm{d}\left(\mathrm{z}^{2}\right)\right] \rightarrow \mathrm{C}\left(\mathrm{sp}^{2}\right)^{*}+\pi^{*}(1)$ & 0.0038 \\
21221101 & $\mathrm{~d}(\mathrm{yz})+\mathrm{d}\left(\mathrm{x}^{2}-\mathrm{y}^{2}\right) \rightarrow \mathrm{C}\left(\mathrm{sp}^{2}\right)^{*}+\pi^{*}(2)$ & 0.0028 \\
\hline
\end{tabular}


Table S2J. Compound 2 - CASSCF/QD-NEVPT2 composition of the formal Ni(I) ground state while varying number of doublet roots. 8 o, 9e Active Space -25 quartet roots, $X$ doublet roots (where $X$ ranged from 15 to 45 ).

\begin{tabular}{|c|c|c|c|c|c|}
\hline \multicolumn{6}{|c|}{ CI-Vector Weights (\# Quartets / \# Doublets) } \\
\hline Transition & 25/15 CPCM & 25/15 Gas & 25/25 Gas & 25/35 Gas & 25/45 Gas \\
\hline $\mathrm{d}(\mathrm{xz}) \rightarrow \pi^{*}(1)$ & 0.6210 & 0.6788 & 0.6610 & 0.6567 & 0.6650 \\
\hline $\mathrm{d}\left(\mathrm{x}^{2}-\mathrm{y}^{2}\right) \rightarrow \pi^{*}(1)$ & 0.0887 & 0.0995 & 0.1219 & 0.1153 & 0.1245 \\
\hline formal $\mathrm{Ni}(\mathrm{I})$ & 0.0671 & 0.0691 & 0.0680 & 0.0707 & 0.0693 \\
\hline \multicolumn{6}{|c|}{ Doublet $\rightarrow$ Doublet Transition Energies $\left(\mathrm{kcal} \mathrm{mol}^{-1}\right)$} \\
\hline Transition & 25/15 CPCM & 25/15 Gas & 25/25 Gas & 25/35 Gas & 25/45 Gas \\
\hline 1 & 5.1 & 6.1 & 6.3 & 6.6 & 6.6 \\
\hline 2 & 7.4 & 7.7 & 7.8 & 8.0 & 8.0 \\
\hline 3 & 9.6 & 9.3 & 8.6 & 8.9 & 8.6 \\
\hline 4 & 26.8 & 27.0 & 25.8 & 26.2 & 26.3 \\
\hline 5 & 28.8 & 29.1 & 29.2 & 29.4 & 29.5 \\
\hline 6 & 32.4 & 33.3 & 33.7 & 34.0 & 34.1 \\
\hline 7 & 35.2 & 35.6 & 35.4 & 35.9 & 35.6 \\
\hline 8 & 37.0 & 37.9 & 35.8 & 36.2 & 36.3 \\
\hline 9 & 38.6 & 39.0 & 38.2 & 38.5 & 38.6 \\
\hline 10 & 41.2 & 41.3 & 39.3 & 39.6 & 39.6 \\
\hline 11 & 49.7 & 50.6 & 39.4 & 39.8 & 39.7 \\
\hline 12 & 50.8 & 51.7 & 41.6 & 42.0 & 42.1 \\
\hline 13 & 55.1 & 56.1 & 49.7 & 49.9 & 49.9 \\
\hline 14 & 57.3 & 58.7 & 50.8 & 51.1 & 50.9 \\
\hline
\end{tabular}


Table S2K. Leadings terms of CI-vector for the $\mathrm{T}_{0}$ (first triplet manifold reached from vertical excitation of singlet ground state) of $\mathbf{2}$ in Figure S2J. Just past the equilibrium geometry, the CIvector is dominated by ${ }^{3} \mathrm{~d}-\mathrm{d}$ transitions. Note that using the relaxed, DFT optimized $C_{4 \mathrm{v}}$ triplet geometries of 2 (surfaces shown in Figure S2G) the ground state triplet CI-vector is very heavily dominated by the $\left[\mathrm{d}\left(\mathrm{x}^{2}-\mathrm{y}^{2}\right)\right]^{1}\left[\mathrm{~d}\left(\mathrm{z}^{2}\right) / \mathrm{C}\left(\mathrm{sp}^{2}\right)^{*}\right]^{1}{ }^{3} \mathrm{~d}-\mathrm{d}$ transition $(64 \%$ at the $1.95 \AA$ equilibrium geometry, Figure S2I bottom). At long Ni-C distances, the transition $\mathrm{d}(\mathrm{xz}) \rightarrow \pi^{*}(1)$ is becomes dominant, making for a $[\mathrm{d}(\mathrm{xz})]^{1}\left[\mathrm{~d}\left(\mathrm{x}^{2}-\mathrm{y}^{2}\right)\right]^{1}\left[\left(\mathrm{dz} \mathrm{z}^{2}\right) / \mathrm{C}\left(\mathrm{sp}^{2}\right)^{*}\right]^{1}\left[\pi^{*}(1)\right]^{1}$ configuration.

\begin{tabular}{|c|c|c|}
\hline Ni-C Distance $(\AA)$ & Weight & Transition \\
\hline \multirow[t]{2}{*}{1.89 (equil.) } & 0.8168 & $\mathrm{~d}\left(\mathrm{z}^{2}\right) \rightarrow \pi^{*}(1)$ \\
\hline & 0.0659 & $2 \mathrm{x}\left[\right.$ bonding $\left.\mathrm{d}\left(\mathrm{x}^{2}-\mathrm{y}^{2}\right) / \mathrm{C}\left(\mathrm{sp}^{2}\right)\right]+\mathrm{d}\left(\mathrm{z}^{2}\right) \rightarrow 2 \mathrm{x}\left[\mathrm{d}\left(\mathrm{x}^{2}-\mathrm{y}^{2}\right) / \mathrm{C}\left(\mathrm{sp}^{2}\right)^{*}\right]+\pi^{*}(1)$ \\
\hline \multirow[t]{2}{*}{2.02} & 0.4339 & $\mathrm{~d}\left(\mathrm{z}^{2}\right) \rightarrow \mathrm{d}\left(\mathrm{x}^{2}-\mathrm{y}^{2}\right) / \mathrm{C}\left(\mathrm{sp}^{2}\right)^{*}$ \\
\hline & 0.2551 & bonding $\mathrm{d}\left(\mathrm{x}^{2}-\mathrm{y}^{2}\right) / \mathrm{C}\left(\mathrm{sp}^{2}\right) \rightarrow \mathrm{d}\left(\mathrm{x}^{2}-\mathrm{y}^{2}\right) / \mathrm{C}\left(\mathrm{sp}^{2}\right)^{*}$ \\
\hline \multirow[t]{2}{*}{2.21} & 0.3666 & bonding $\mathrm{d}\left(\mathrm{x}^{2}-\mathrm{y}^{2}\right) / \mathrm{C}\left(\mathrm{sp}^{2}\right) \rightarrow \mathrm{d}\left(\mathrm{x}^{2}-\mathrm{y}^{2}\right) / \mathrm{C}\left(\mathrm{sp}^{2}\right)^{*}$ \\
\hline & 0.2786 & $\mathrm{~d}\left(\mathrm{z}^{2}\right) \rightarrow \mathrm{d}\left(\mathrm{x}^{2}-\mathrm{y}^{2}\right) / \mathrm{C}\left(\mathrm{sp}^{2}\right)^{*}$ \\
\hline \multirow[t]{2}{*}{2.40} & 0.5334 & $\mathrm{~d}\left(\mathrm{z}^{2}\right) \rightarrow \mathrm{d}\left(\mathrm{x}^{2}-\mathrm{y}^{2}\right) / \mathrm{C}\left(\mathrm{sp}^{2}\right)^{*}$ \\
\hline & 0.3130 & $\mathrm{~d}\left(\mathrm{z}^{2}\right)+$ bonding $\mathrm{d}\left(\mathrm{x}^{2}-\mathrm{y}^{2}\right) / \mathrm{C}\left(\mathrm{sp}^{2}\right) \rightarrow 2 \mathrm{x}\left[\mathrm{d}\left(\mathrm{x}^{2}-\mathrm{y}^{2}\right) / \mathrm{C}\left(\mathrm{sp}^{2}\right)^{*}\right]$ \\
\hline \multirow[t]{2}{*}{2.60} & 0.4218 & $\mathrm{~d}\left(\mathrm{z}^{2}\right) \rightarrow \pi^{*}(1)$ \\
\hline & 0.3788 & $\mathrm{~d}\left(\mathrm{z}^{2}\right)+$ bonding $\mathrm{d}\left(\mathrm{x}^{2}-\mathrm{y}^{2}\right) / \mathrm{C}\left(\mathrm{sp}^{2}\right) \rightarrow \mathrm{d}\left(\mathrm{x}^{2}-\mathrm{y}^{2}\right) / \mathrm{C}\left(\mathrm{sp}^{2}\right)^{*}+\pi^{*}(1)$ \\
\hline \multirow[t]{2}{*}{2.91} & 0.6157 & $\mathrm{~d}\left(\mathrm{z}^{2}\right)+$ bonding $\mathrm{d}\left(\mathrm{x}^{2}-\mathrm{y}^{2}\right) / \mathrm{C}\left(\mathrm{sp}^{2}\right) \rightarrow \mathrm{d}\left(\mathrm{x}^{2}-\mathrm{y}^{2}\right) / \mathrm{C}\left(\mathrm{sp}^{2}\right)^{*}+\pi^{*}(1)$ \\
\hline & 0.2267 & $\mathrm{~d}\left(\mathrm{z}^{2}\right) \rightarrow \pi^{*}(1)$ \\
\hline \multirow[t]{2}{*}{3.14} & 0.4414 & $\mathrm{~d}(\mathrm{xz})+$ bonding $\mathrm{d}\left(\mathrm{x}^{2}-\mathrm{y}^{2}\right) / \mathrm{C}\left(\mathrm{sp}^{2}\right) \rightarrow \mathrm{d}\left(\mathrm{x}^{2}-\mathrm{y}^{2}\right) / \mathrm{C}\left(\mathrm{sp}^{2}\right)^{*}+\pi^{*}(1)$ \\
\hline & 0.0777 & $\mathrm{~d}\left(\mathrm{z}^{2}\right)+$ bonding $\mathrm{d}\left(\mathrm{x}^{2}-\mathrm{y}^{2}\right) / \mathrm{C}\left(\mathrm{sp}^{2}\right) \rightarrow \mathrm{d}\left(\mathrm{x}^{2}-\mathrm{y}^{2}\right) / \mathrm{C}\left(\mathrm{sp}^{2}\right)^{*}+\pi^{*}(1)$ \\
\hline \multirow[t]{2}{*}{3.31} & 0.5253 & $\mathrm{~d}(\mathrm{xz})+$ bonding $\mathrm{d}\left(\mathrm{x}^{2}-\mathrm{y}^{2}\right) / \mathrm{C}\left(\mathrm{sp}^{2}\right) \rightarrow \mathrm{d}\left(\mathrm{x}^{2}-\mathrm{y}^{2}\right) / \mathrm{C}\left(\mathrm{sp}^{2}\right)^{*}+\pi^{*}(1)$ \\
\hline & 0.1070 & $2 \mathrm{x}\left[\right.$ bonding $\left.\mathrm{d}\left(\mathrm{x}^{2}-\mathrm{y}^{2}\right) / \mathrm{C}\left(\mathrm{sp}^{2}\right)\right] \rightarrow \mathrm{d}\left(\mathrm{x}^{2}-\mathrm{y}^{2}\right) / \mathrm{C}\left(\mathrm{sp}^{2}\right)^{*}+\pi^{*}(1)$ \\
\hline \multirow[t]{2}{*}{3.49} & 0.6173 & $\mathrm{~d}(\mathrm{xz})+$ bonding $\mathrm{d}\left(\mathrm{x}^{2}-\mathrm{y}^{2}\right) / \mathrm{C}\left(\mathrm{sp}^{2}\right) \rightarrow \mathrm{d}\left(\mathrm{x}^{2}-\mathrm{y}^{2}\right) / \mathrm{C}\left(\mathrm{sp}^{2}\right)^{*}+\pi^{*}(1)$ \\
\hline & 0.1150 & $2 \mathrm{x}\left[\right.$ bonding $\left.\mathrm{d}\left(\mathrm{x}^{2}-\mathrm{y}^{2}\right) / \mathrm{C}\left(\mathrm{sp}^{2}\right)\right] \rightarrow \mathrm{d}\left(\mathrm{x}^{2}-\mathrm{y}^{2}\right) / \mathrm{C}\left(\mathrm{sp}^{2}\right)^{*}+\pi^{*}(1)$ \\
\hline \multirow[t]{2}{*}{3.71} & 0.2595 & $\mathrm{~d}(\mathrm{yz})+$ bonding $\mathrm{d}\left(\mathrm{x}^{2}-\mathrm{y}^{2}\right) / \mathrm{C}\left(\mathrm{sp}^{2}\right) \rightarrow \mathrm{d}\left(\mathrm{x}^{2}-\mathrm{y}^{2}\right) / \mathrm{C}\left(\mathrm{sp}^{2}\right)^{*}+\pi^{*}(1)$ \\
\hline & 0.1641 & $2 \mathrm{x}\left[\right.$ bonding $\left.\mathrm{d}\left(\mathrm{x}^{2}-\mathrm{y}^{2}\right) / \mathrm{C}\left(\mathrm{sp}^{2}\right)\right] \rightarrow \mathrm{d}\left(\mathrm{x}^{2}-\mathrm{y}^{2}\right) / \mathrm{C}\left(\mathrm{sp}^{2}\right)^{*}+\pi^{*}(1)$ \\
\hline \multirow[t]{2}{*}{3.94} & 0.2298 & $\mathrm{~d}(\mathrm{yz})+$ bonding $\mathrm{d}\left(\mathrm{x}^{2}-\mathrm{y}^{2}\right) / \mathrm{C}\left(\mathrm{sp}^{2}\right) \rightarrow \mathrm{d}\left(\mathrm{x}^{2}-\mathrm{y}^{2}\right) / \mathrm{C}\left(\mathrm{sp}^{2}\right)^{*}+\pi^{*}(1)$ \\
\hline & 0.2117 & $\mathrm{~d}(\mathrm{xz})+$ bonding $\mathrm{d}\left(\mathrm{x}^{2}-\mathrm{y}^{2}\right) / \mathrm{C}\left(\mathrm{sp}^{2}\right) \rightarrow \mathrm{d}\left(\mathrm{x}^{2}-\mathrm{y}^{2}\right) / \mathrm{C}\left(\mathrm{sp}^{2}\right)^{*}+\pi^{*}(1)$ \\
\hline
\end{tabular}


Table S2L. Spin Contamination Values From BSS Calculation and Yamaguchi Correction ${ }^{1,2}$ BSS Energy of 2. Using $<\mathrm{S}^{2}>$ values and energies for the triplet and BSS solutions for the same geometry, $\left.\left.\alpha=\left\langle\mathrm{S}^{2}\right\rangle_{\text {trip. }} /\left(\left\langle\mathrm{S}^{2}\right\rangle_{\text {trip. }}-\mathrm{S}^{2}\right\rangle_{\mathrm{BSS}}\right), \beta=\left\langle\mathrm{S}^{2}\right\rangle_{\mathrm{BSS}} /\left(\left\langle\mathrm{S}^{2}\right\rangle_{\text {trip. }}-\mathrm{S}^{2}\right\rangle_{\mathrm{BSS}}\right)$, and $\mathrm{E}_{\text {Yamaguchi }}=$ $\alpha \mathrm{E}_{\text {BSS }}-\beta \mathrm{E}_{\text {trip }}$.

\begin{tabular}{|c|c|c|c|c|c|c|c|}
\hline $\begin{array}{l}\text { Ni-C } \\
\text { Dist. } \\
(\AA ̊) \\
\end{array}$ & $\begin{array}{l}<S^{2}> \\
S=1\end{array}$ & $\begin{array}{l}<S^{2}> \\
\text { BSS }\end{array}$ & $\alpha$ & $\boldsymbol{\beta}$ & $\begin{array}{c}\text { Energy } \\
\mathrm{S}=1 / \\
{\text { kcal } \mathrm{mol}^{-1}}\end{array}$ & $\begin{array}{c}\text { Energy } \\
\text { BSS/ } \\
\text { kcal mol }^{-1} \\
\end{array}$ & $\begin{array}{c}\text { Energy } \\
\text { Yamaguchi/ } \\
\text { kcal mol }^{-1}\end{array}$ \\
\hline 1.89 & 2.01 & 0.00 & 1.00 & 0.00 & 37.5 & 0.0 & 0.0 \\
\hline 2.02 & 2.01 & 0.00 & 1.00 & 0.00 & 34.6 & 1.5 & 1.5 \\
\hline 2.21 & 2.01 & 0.00 & 1.00 & 0.00 & 36.7 & 8.8 & 8.8 \\
\hline 2.40 & 2.01 & 0.00 & 1.00 & 0.00 & 41.7 & 18.3 & 18.3 \\
\hline 2.60 & 2.01 & 0.03 & 1.02 & 0.02 & 47.0 & 27.6 & 27.2 \\
\hline 2.91 & 2.04 & 0.50 & 1.33 & 0.33 & 51.7 & 39.6 & 35.6 \\
\hline 3.14 & 2.04 & 0.94 & 1.86 & 0.86 & 42.9 & 44.5 & 45.9 \\
\hline 3.31 & 2.04 & 0.98 & 1.93 & 0.93 & 44.3 & 45.4 & 46.4 \\
\hline 3.49 & 2.03 & 1.00 & 1.98 & 0.98 & 44.8 & 45.5 & 46.2 \\
\hline 3.71 & 2.03 & 1.02 & 2.00 & 1.00 & 44.8 & 45.3 & 45.7 \\
\hline 3.94 & 2.03 & 1.02 & 2.02 & 1.02 & 44.6 & 44.9 & 45.1 \\
\hline
\end{tabular}




\section{$\underline{\text { E. Figures Part 2: Compound 2 }}$}
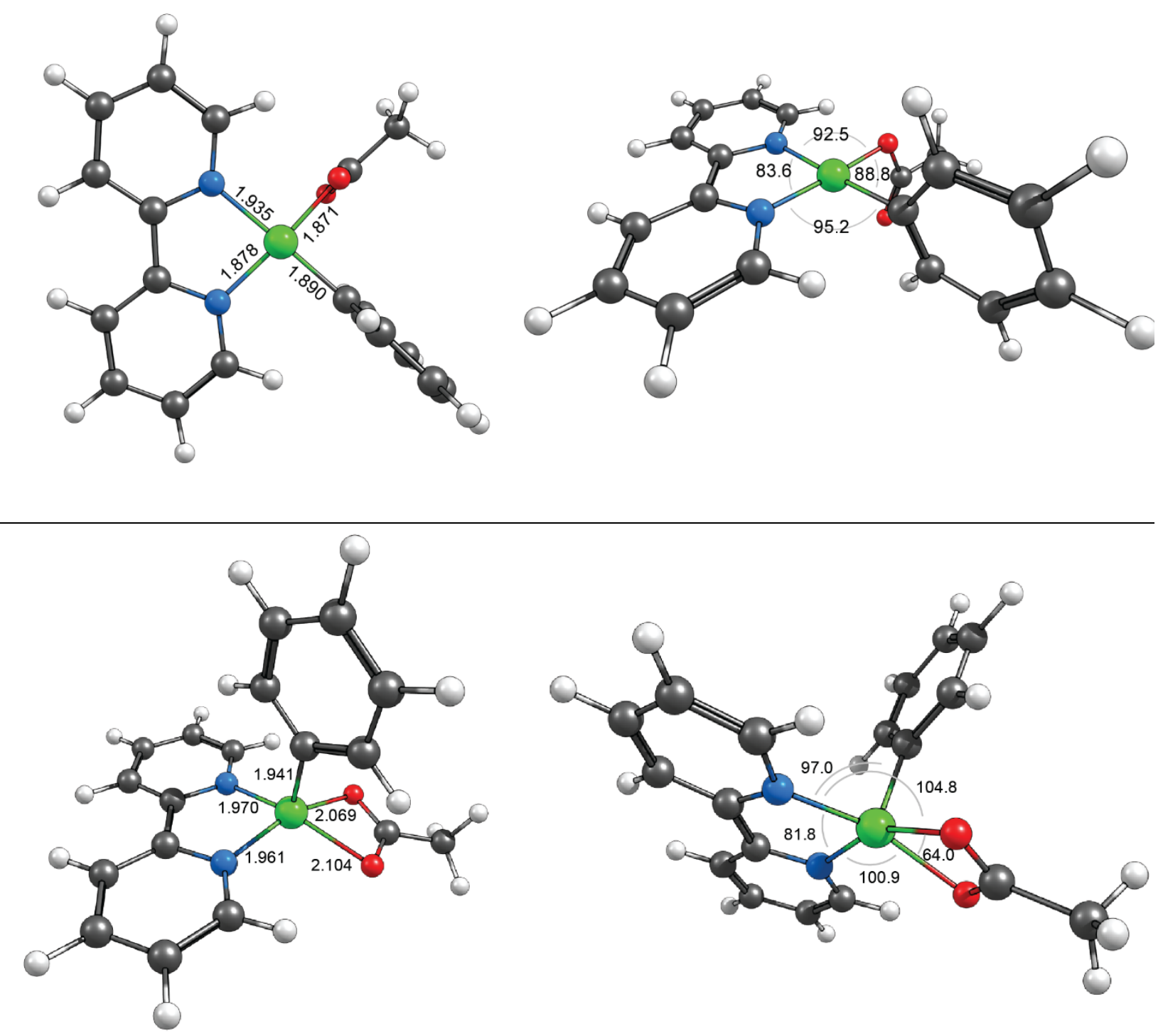

Figure S2A. Top: square planar equilibrium geometry of compound $2(\mathrm{~S}=0)$, and bottom: square pyramidal equilibrium geometry of $2(\mathrm{~S}=1)$ as calculated by DFT. Selected bond lengths and angles are shown. 


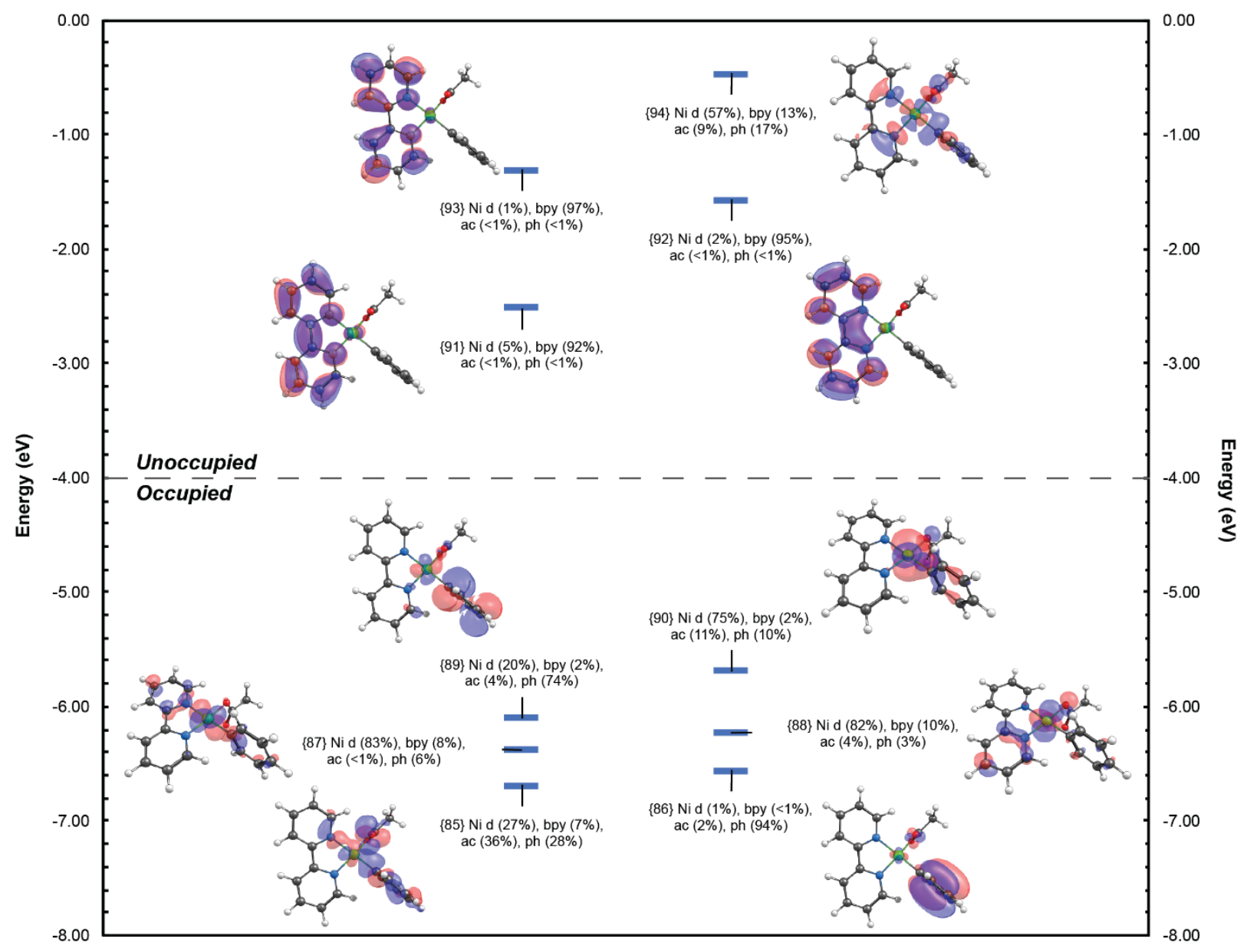

Figure S2B. Compound $2(\mathrm{~S}=0)$ - Single point DFT Frontier molecular orbital diagram. Orbitals are offset for clarity. 


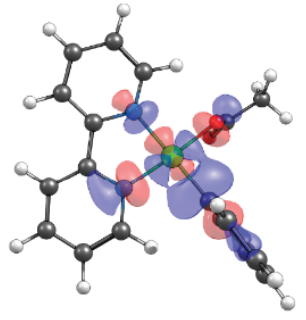

$\mathrm{d}\left(\mathbf{x}^{2}-\mathrm{y}^{2}\right) / \mathrm{C}\left(\mathbf{s p}^{2}\right)^{*}$

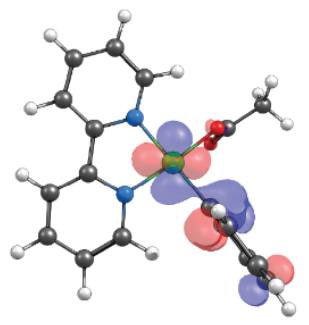

$\mathbf{d}(\mathbf{x y})$
Occ: 1.761

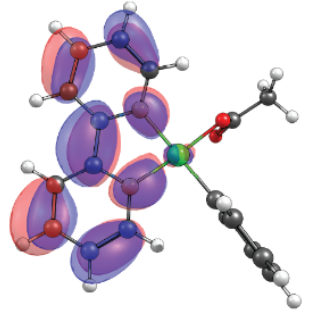

$\pi^{*}(1)$

Occ: 0.395

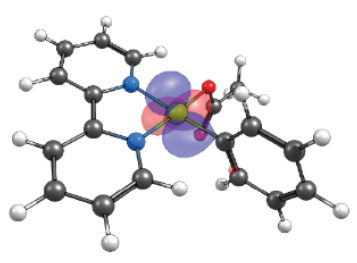

$\mathbf{d}(\mathbf{x z})$
Occ: 1.742

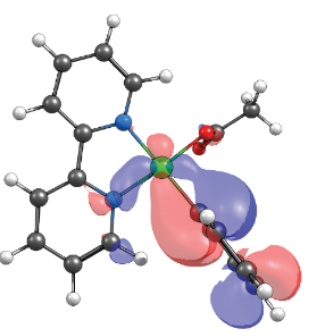

bonding $d(x y) / C(\pi)$

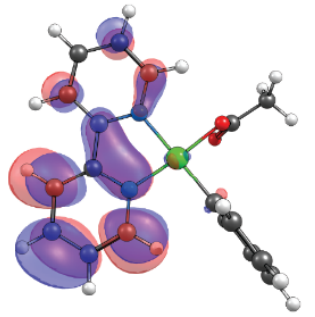

$\pi^{*}(2)$

Occ: 0.183

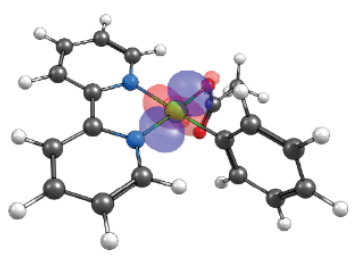

$\mathbf{d}(\mathbf{y z})$
Occ: 1.696

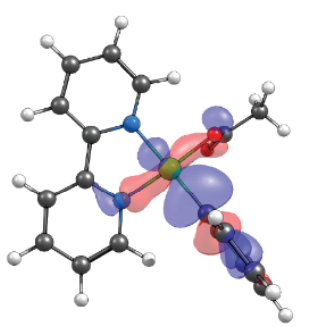

bonding $\mathbf{d}\left(\mathbf{x}^{2}-\mathbf{y}^{2}\right) / \mathbf{C}\left(\mathbf{s p}^{2}\right)$
$O c C: 1.807$

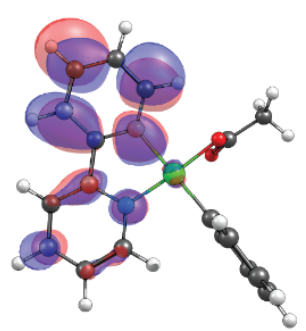

$\pi^{*}(3)$

Occ: 0.130

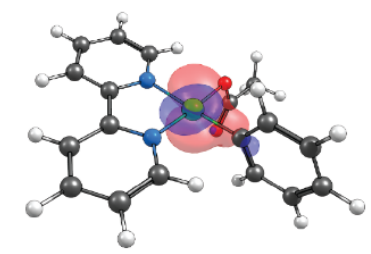

$d\left(z^{2}\right)$

Figure S2C. Compound 2 ( $\mathrm{S}=0$ ) - CASSCF/QD-NEVPT2 10o, 12e Active Space. 


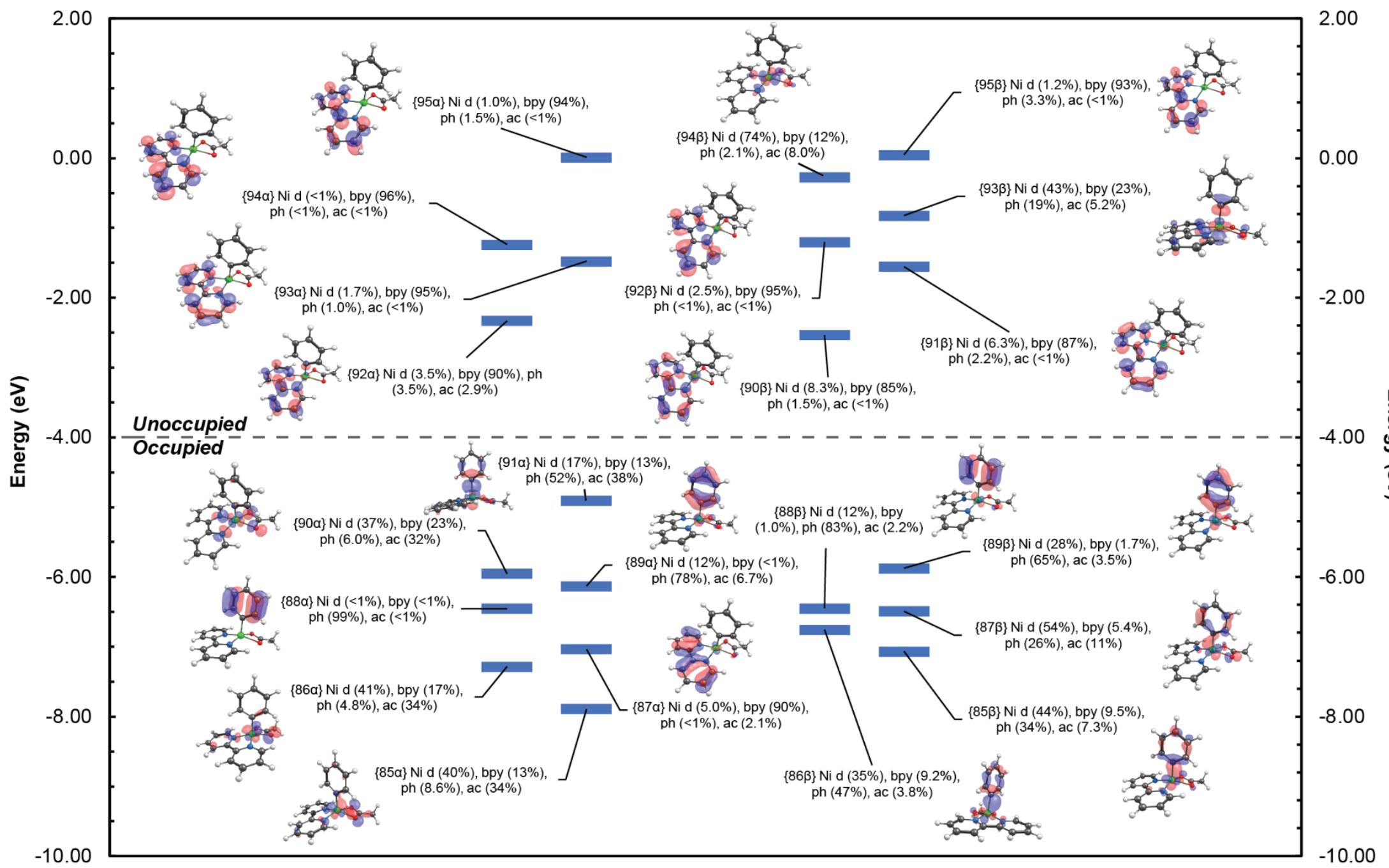

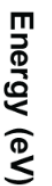

Figure S2D. Compound $2(\mathrm{~S}=1)$ - Single point DFT Frontier molecular orbital diagram, with $\alpha$ orbitals on the left and $\beta$ orbitals on the right. $\alpha$ and $\beta$ orbitals are also offset for clarity. 


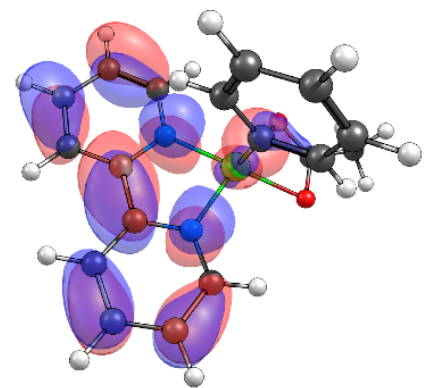

$\pi^{*}(1)$

Occ: 0.509

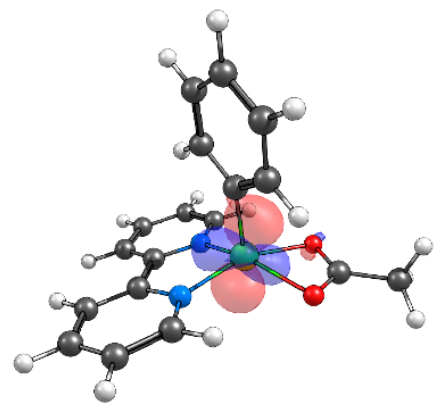

$d(y z)$

Occ: 1.698

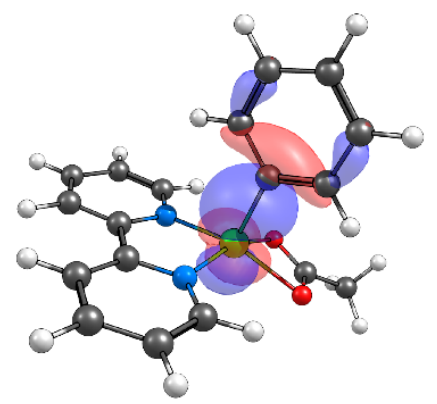

bonding $\mathrm{d}\left(\mathrm{z}^{2}\right) / \mathrm{C}\left(\mathbf{s p}^{2}\right)$ Occ: 1.726

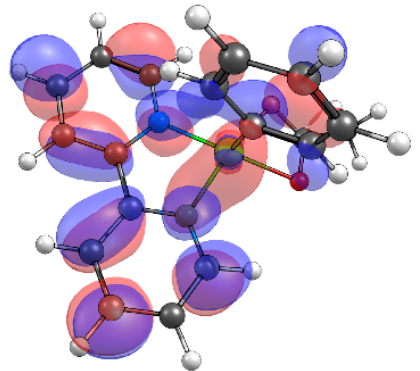

$\pi^{*}(2)$

Occ: 0.066

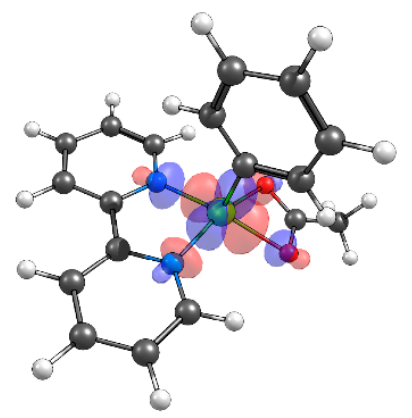

$$
\mathrm{d}\left(\mathrm{x}^{2}-\mathrm{y}^{2}\right)
$$

Occ: 1.385

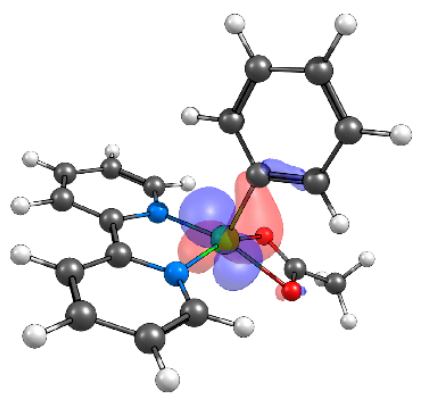

$d(x z)$

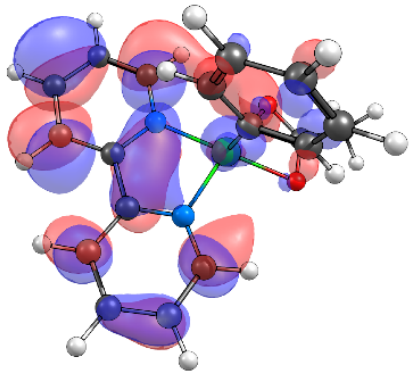

$\pi^{*}(3)$

Occ: 0.059

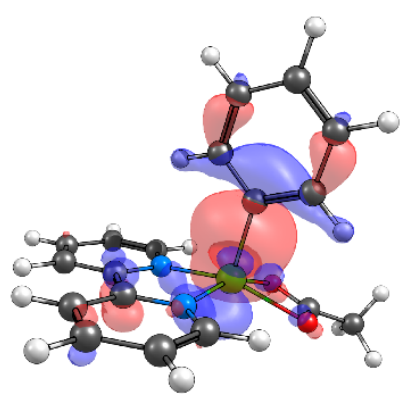

$\mathrm{d}\left(\mathbf{z}^{2}\right) / \mathbf{C}\left(\mathbf{s p}^{2}\right)^{*}$

Occ: 0.143

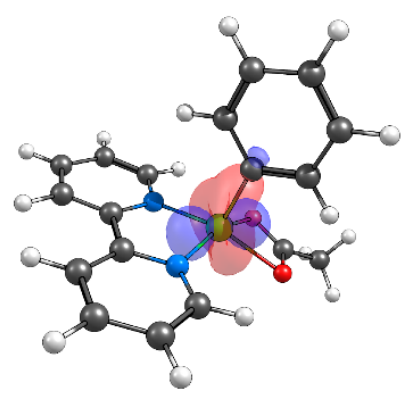

$d(x y)$

Occ: 1.703

Figure S2E. Compound 2 ( $\mathrm{S}=1)$ - CASSCF/QD-NEVPT2 9o, 10e Active Space. 


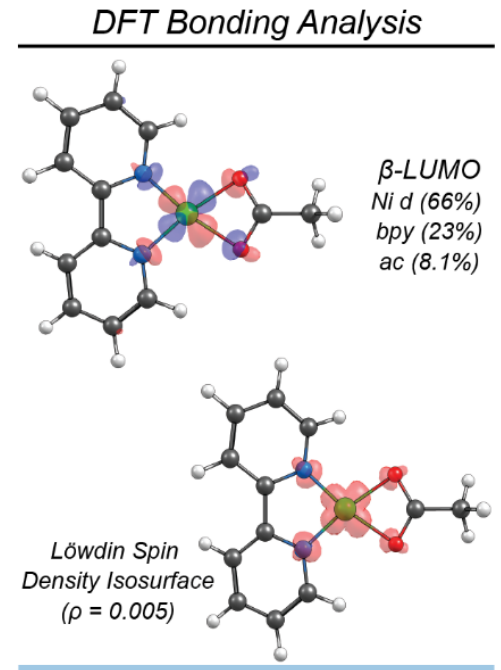

Ni (0.833), bpy (0.102), ac (0.065)
CASSCF/QD-NEVPT2 (8 orbitals, 9 electrons) 25 quartets, 15 doublets

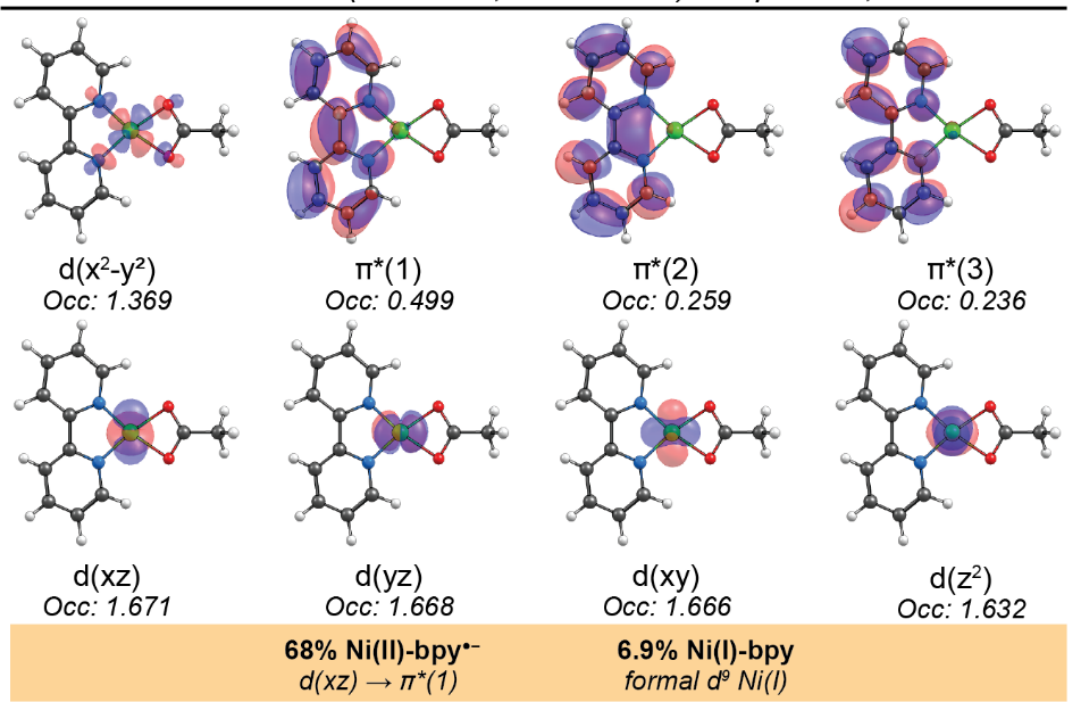

Figure S2F. Comparison between the description given by DFT vs CASSCF/QD-NEVPT2 of the formal $\mathrm{Ni}(\mathrm{I})$ product of 2 that results after $\mathrm{Ni}-\mathrm{C}$ bond cleavage.

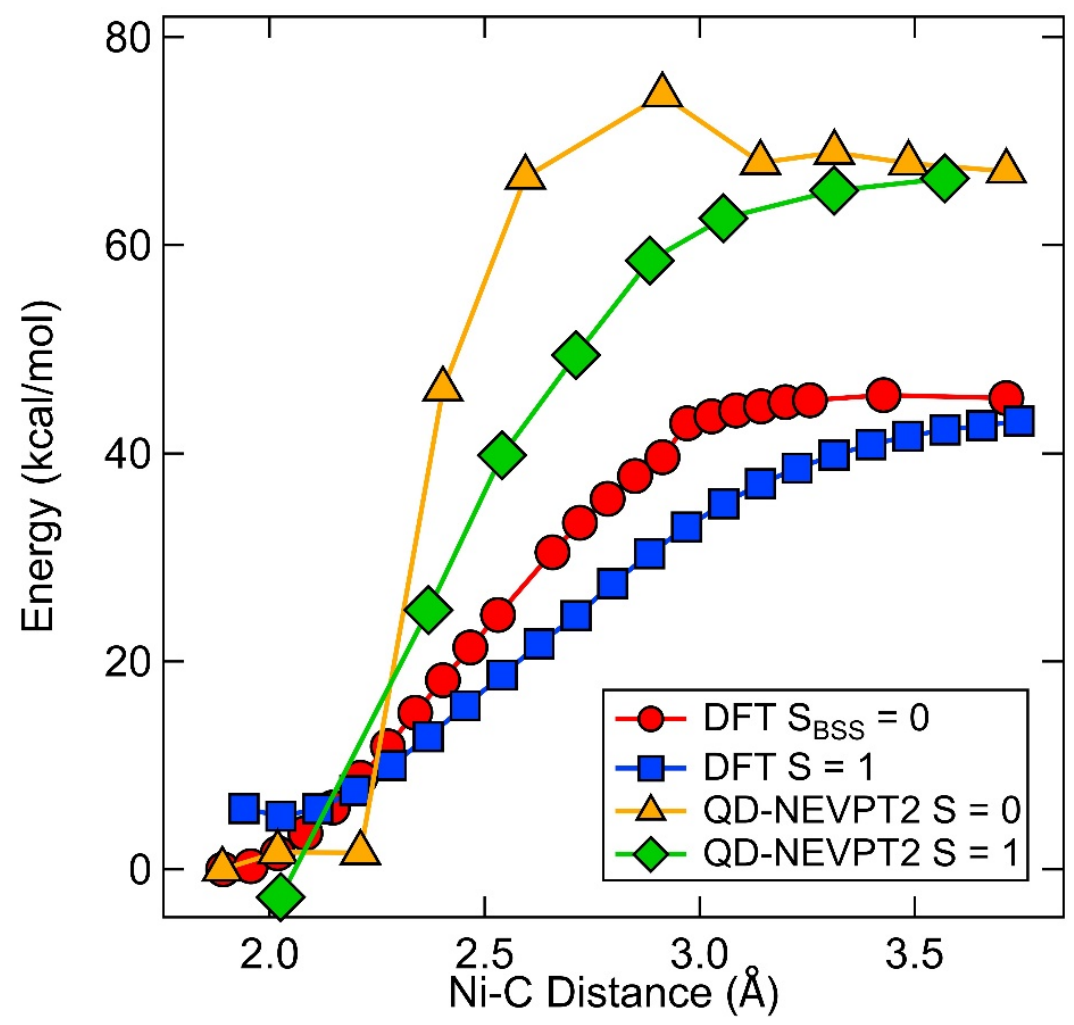

Figure S2G. Thermodynamics (DFT and CASSCF/QDNEVPT2) along Ni-C coordinate for 2. 

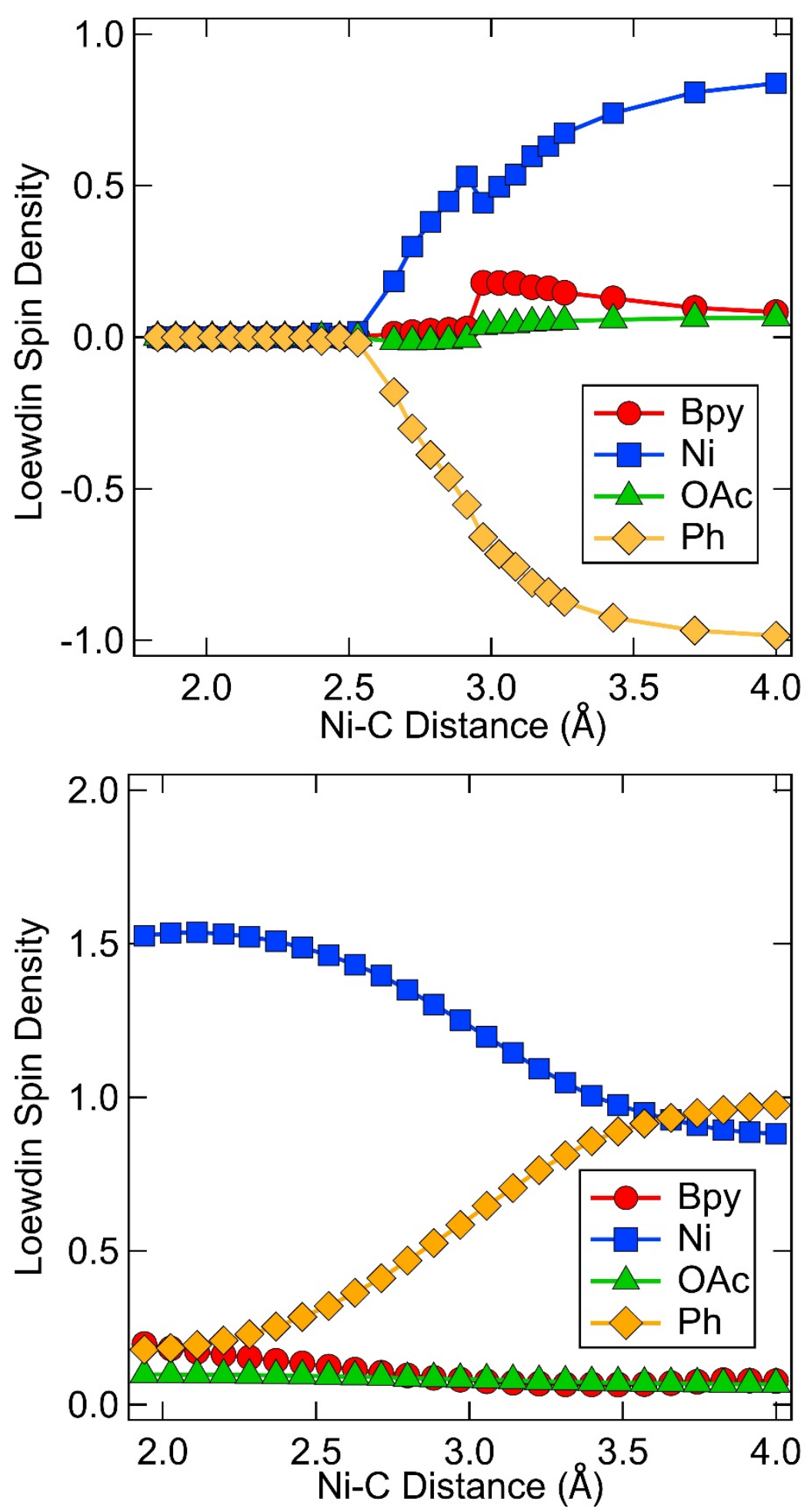

Figure S2H. DFT Loewdin spin density per group along the Ni-C coordinate for 2: (top) BSS and (bottom) UKS S=1. 

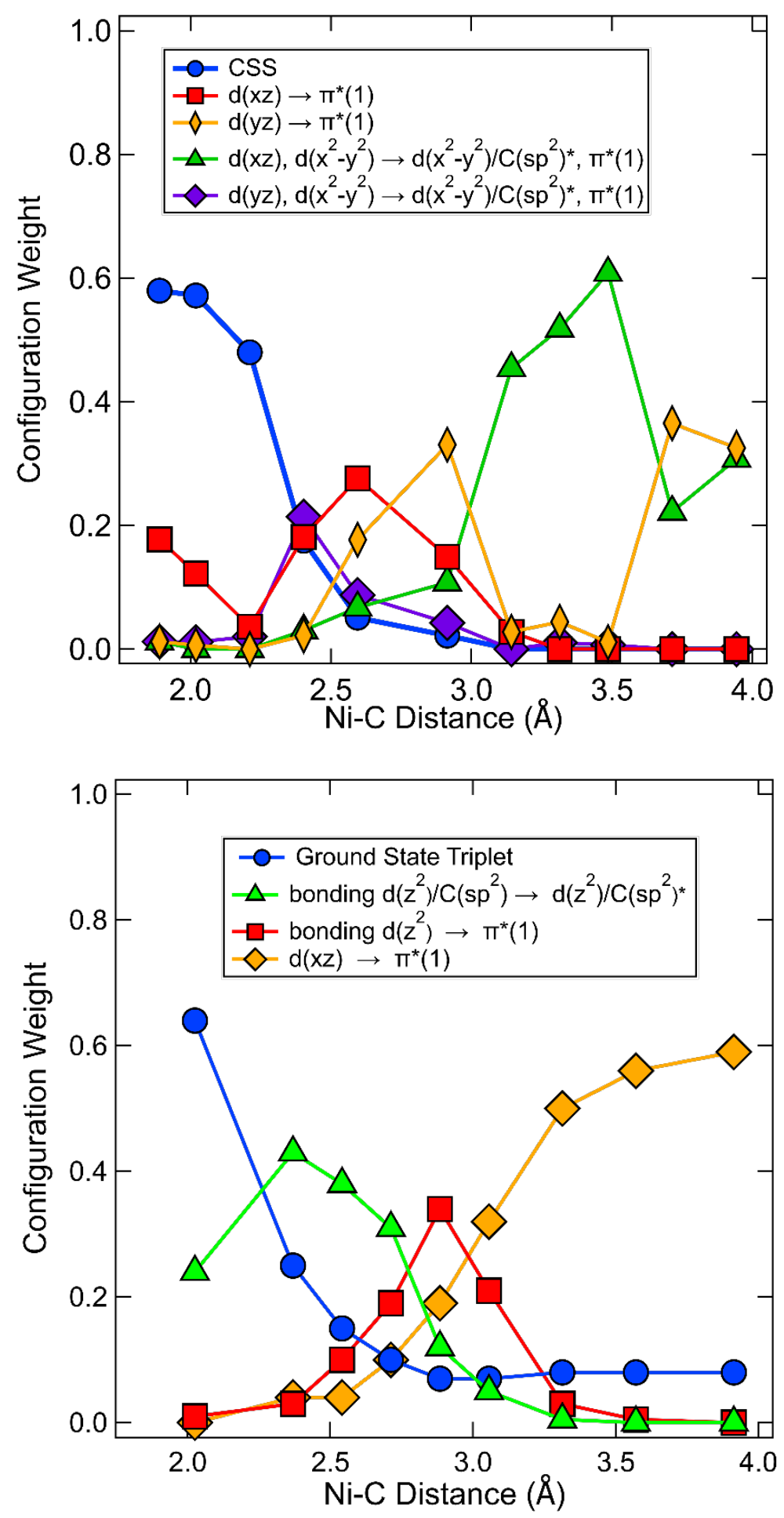

Figure S2I. Dominant CASSCF/QD-NEVPT2 CI vectors along the $\mathrm{Ni}-\mathrm{C}$ coordinate for 2: (top) $\mathrm{S}=0$ and (bottom) $\mathrm{S}=1$. Note that for high spin 2, the ground state triplet is $\left[\mathrm{d}\left(\mathrm{x}^{2}-\mathrm{y}^{2}\right)\right]^{1}\left[\left(\mathrm{dz}^{2}\right) / \mathrm{C}\left(\mathrm{sp}^{2}\right)^{*}\right]^{1}$, making a $\mathrm{d}(\mathrm{xz}) \rightarrow \pi^{*}(1)$ transition yield a configuration of $[\mathrm{d}(\mathrm{xz})]^{1}\left[\mathrm{~d}\left(\mathrm{x}^{2}-\mathrm{y}^{2}\right)\right]^{1}\left[\left(\mathrm{dz} \mathrm{z}^{2}\right) / \mathrm{C}\left(\mathrm{sp}^{2}\right)^{*}\right]^{1}\left[\pi^{*}(1)\right]^{1}$. 


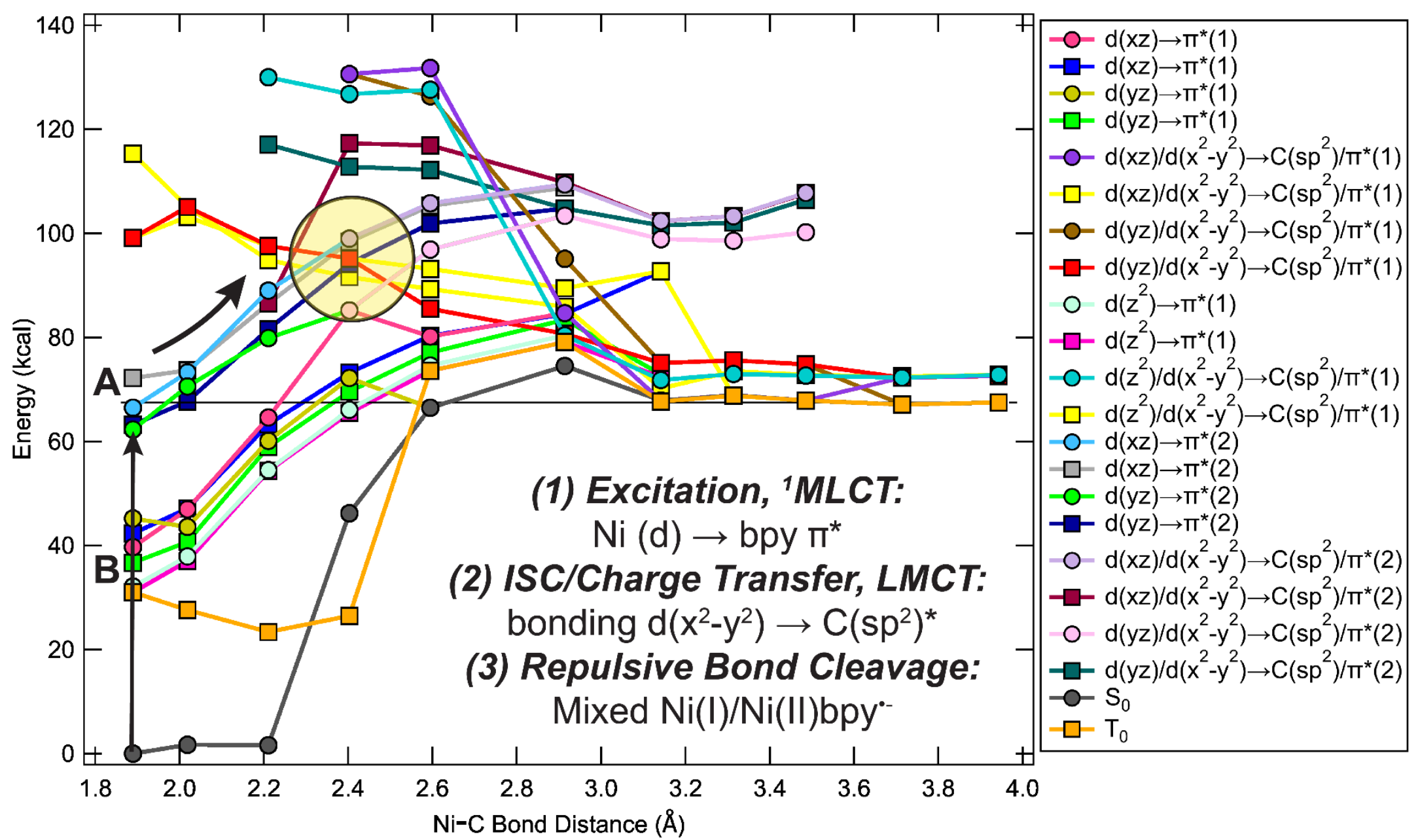

Figure S2J. CASSCF/QD-NEVPT2 calculated relaxed ground and excited state PESs along the Ni-C coordinate of 2, with depictions of vertical excitation (black vertical arrow), the higher (A) and lower energy (B) manifolds of MLCTs, and the crossings between the higher energy MLCTs and the repulsive triplet surfaces (circled). Singlet states are shown with circles, triplets with squares. 


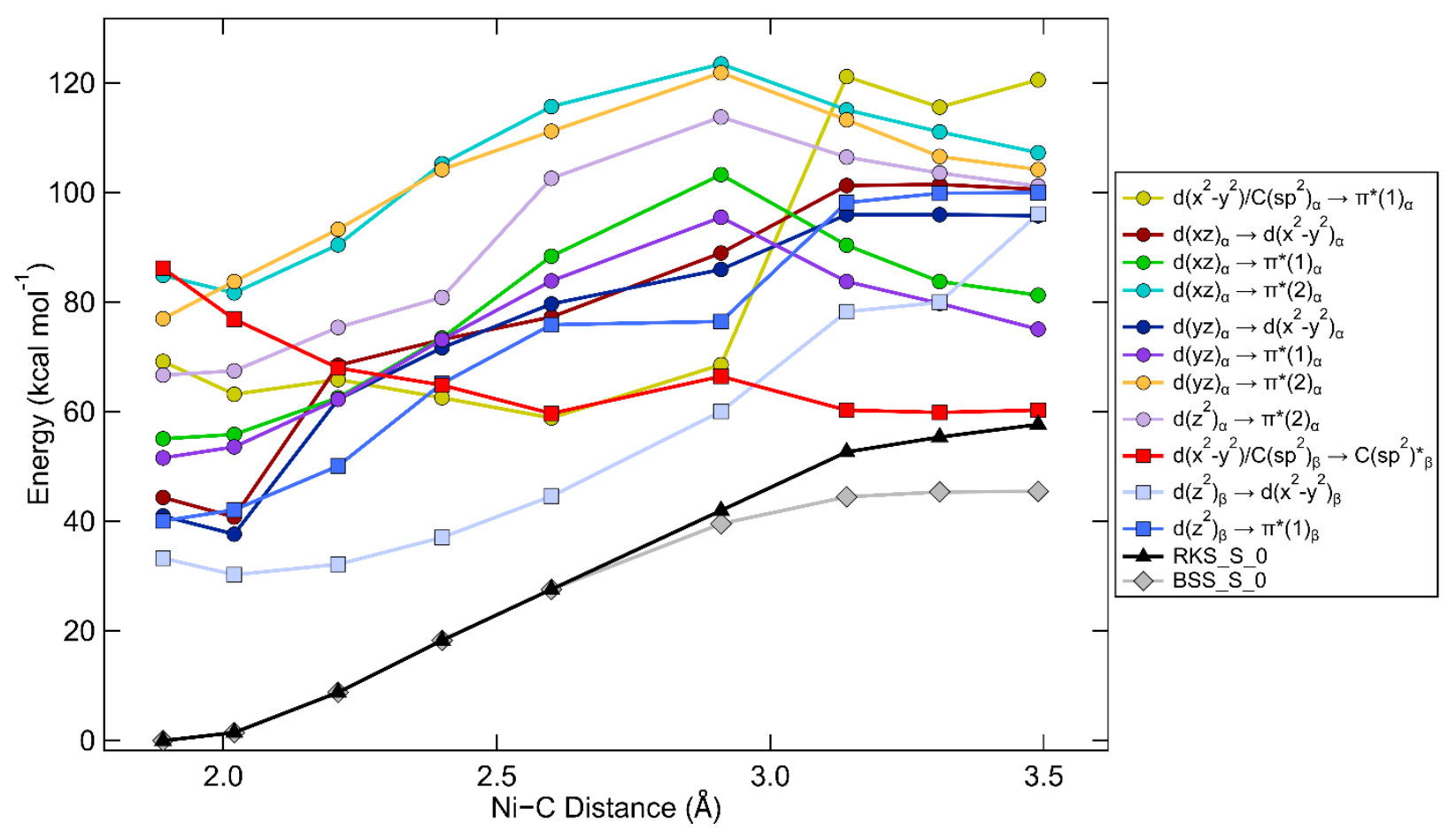

Figure S2K. Broken symmetry singlet TDDFT (CPCM(THF)) excitations along the Ni-C coordinate of 2 show a possible high energy reuplusive state (red line) which is $\sim 86 \mathrm{kcal} \mathrm{mol}^{-1}$ above the equilibrium ground state. Spin-contaminated BSS calculations gave a Ni-C BDE of 45.1

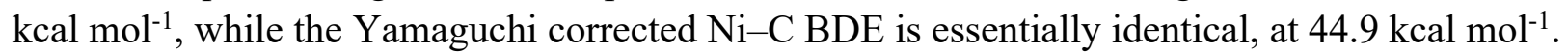

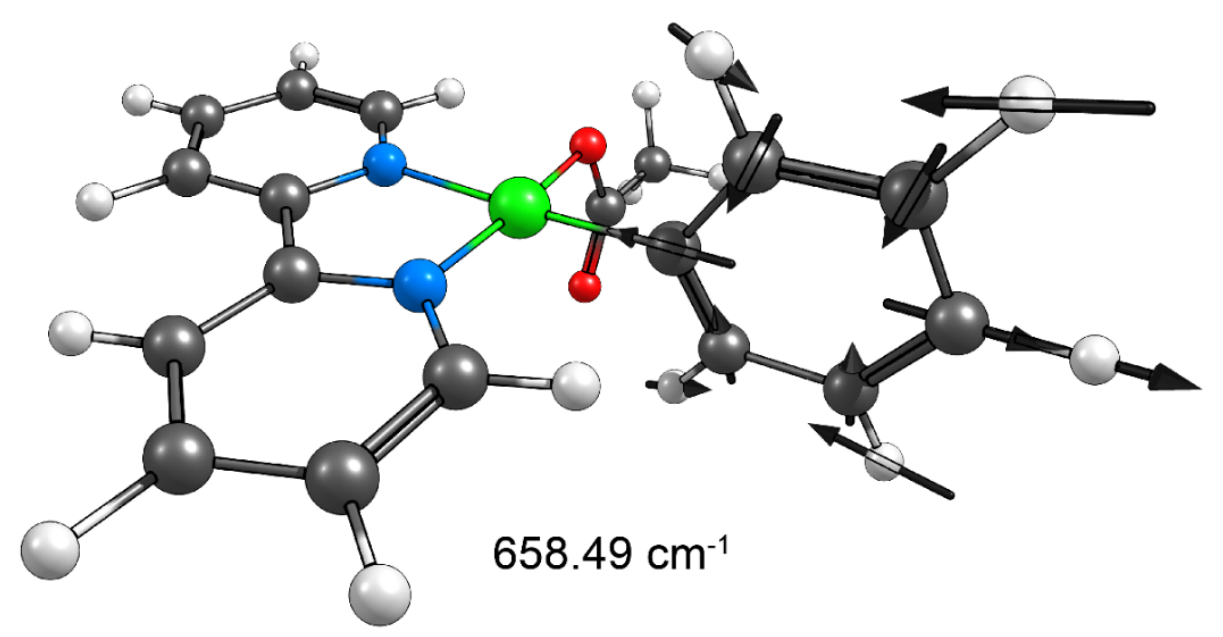

Figure S2L. Higher energy vibrational frequency in $\mathbf{2}$ that exhibits a $\mathrm{Ni}-\mathrm{C}$ bond stretching mode. 


\section{References}

(1) Yamaguchi, K.; Takahara, Y.; Fueno, T.; Houk, K. N. Extended Hartree-Fock (EHF) Theory of Chemical Reactions. Theor. Chim. Acta 1988, 73 (5), 337-364. https://doi.org/10.1007/BF00527740.

(2) Kitagawa, Y.; Saito, T.; Nakanishi, Y.; Kataoka, Y.; Matsui, T.; Kawakami, T.; Okumura, M.; Yamaguchi, K. Spin Contamination Error in Optimized Geometry of Singlet Carbene $\left({ }^{1} \mathrm{~A}_{1}\right)$ by Broken-Symmetry Method. J. Phys. Chem. A 2009, 113 (52), 15041-15046. https://doi.org/10.1021/jp905125g.

(3) Shields, B. J.; Kudisch, B.; Scholes, G. D.; Doyle, A. G. Long-Lived Charge-Transfer States of Nickel(II) Aryl Halide Complexes Facilitate Bimolecular Photoinduced Electron Transfer. J. Am. Chem. Soc. 2018, 140 (8), 3035-3039. https://doi.org/10.1021/jacs.7b13281. 


\section{Appendix: DFT Optimized Structures}

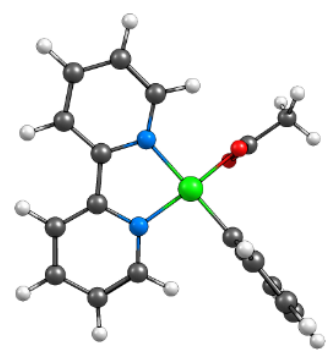

39

Aryl Carboxylate RKS Fully Optimized

$\begin{array}{lrrr}6 & -4.707452000 & -0.391161000 & 0.168879000 \\ 6 & -3.865012000 & -1.497580000 & 0.038873000 \\ 6 & -4.140109000 & 0.887035000 & 0.255790000 \\ 6 & -2.752713000 & 1.016088000 & 0.200045000 \\ 7 & -1.933587000 & -0.047766000 & 0.060734000 \\ 6 & -2.478609000 & -1.298990000 & -0.008334000 \\ 6 & -1.460971000 & -2.349273000 & -0.118250000 \\ 7 & -0.166326000 & -1.869074000 & -0.070411000 \\ 6 & -1.739446000 & -3.714239000 & -0.251538000 \\ 1 & -4.283526000 & -2.504491000 & -0.019809000 \\ 1 & -5.791392000 & -0.525529000 & 0.206513000 \\ 1 & -4.762552000 & 1.777733000 & 0.365224000 \\ 1 & -2.236770000 & 1.976265000 & 0.267990000 \\ 6 & -0.692669000 & -4.632557000 & -0.340510000 \\ 1 & -2.776288000 & -4.054539000 & -0.291989000 \\ 6 & 0.619531000 & -4.146965000 & -0.292636000 \\ 1 & -0.896634000 & -5.700614000 & -0.447096000 \\ 6 & 0.841181000 & -2.778203000 & -0.162330000 \\ 1 & 1.478057000 & -4.818827000 & -0.358493000 \\ 1 & 1.846792000 & -2.360476000 & -0.125912000 \\ 28 & 0.000000000 & 0.000000000 & 0.000000000 \\ 6 & 1.888574000 & 0.000000000 & 0.071467000 \\ 8 & 0.037451000 & 1.868881000 & 0.070582000 \\ 6 & 0.190929000 & 2.451672000 & -1.106243000 \\ 8 & 0.225110000 & 1.869891000 & -2.193488000 \\ 6 & 0.355279000 & 3.966397000 & -1.002699000 \\ 1 & -0.049934000 & 4.368839000 & -0.064071000 \\ 1 & -0.117221000 & 4.453549000 & -1.867090000 \\ 1 & 1.431644000 & 4.201813000 & -1.034290000 \\ 6 & 2.681131000 & 0.110776000 & -1.086655000 \\ 6 & 2.532134000 & -0.141112000 & 1.316068000 \\ 6 & 3.933034000 & -0.191744000 & 1.400485000 \\ 6 & 4.712671000 & -0.100446000 & 0.240291000 \\ 6 & 4.082032000 & 0.055110000 & -1.001171000 \\ 1 & 2.200016000 & 0.268814000 & -2.055522000 \\ 1 & 4.682463000 & 0.143756000 & -1.913297000 \\ 1 & 5.804646000 & -0.139214000 & 0.304678000 \\ 1 & 1.944695000 & -0.207871000 & 2.238925000 \\ 1 & 4.414754000 & -0.298307000 & 2.378800000 \\ & & & \end{array}$

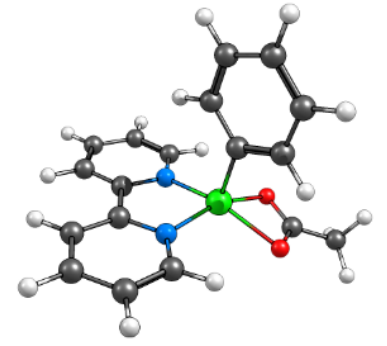

39

Aryl Carboxylate Triplet Fully Optimized

$\begin{array}{llll}\text { C } & -4.628182267 & 0.538495192 & -0.961576888\end{array}$

$\begin{array}{llll}\text { C } & -4.036390125 & -0.716285517 & -0.828856077\end{array}$

$\begin{array}{llll}\text { C } & -3.827429540 & 1.689473586 & -0.844136718\end{array}$

$\begin{array}{llll}\text { C } & -2.466522118 & 1.536018327 & -0.598002976\end{array}$

$\begin{array}{llll}\mathrm{N} & -1.885355862 & 0.321777139 & -0.473131211\end{array}$

$\begin{array}{llll}\text { C } & -2.655468845 & -0.808316711 & -0.582965994\end{array}$

$\begin{array}{llll}\text { C } & -1.899132774 & -2.055341059 & -0.419720306\end{array}$

$\begin{array}{llll}\mathrm{N} & -0.551359209 & -1.866233900 & -0.239020821\end{array}$

$\begin{array}{llll}\text { C } & -2.451445311 & -3.347481997 & -0.428190764\end{array}$

H $\quad-4.642029460 \quad-1.621030291 \quad-0.918182042$

$\begin{array}{llll}\mathrm{H} & -5.700453833 & 0.624361759 & -1.154767210\end{array}$

$\begin{array}{llll}H & -4.254050305 & 2.690045265 & -0.943217439\end{array}$

$\begin{array}{llll}\mathrm{H} & -1.786231644 & 2.385811418 & -0.497655678\end{array}$

C $\quad-1.618281973 \quad-4.452618667 \quad-0.267591134$

H $\quad-3.527480140 \quad-3.485483993 \quad-0.556753202$

$\begin{array}{llll}\text { C } & -0.238083683 & -4.246424965 & -0.090286737\end{array}$

$\begin{array}{llll}\mathrm{H} & -2.034211972 & -5.463163278 & -0.274413057\end{array}$

$\begin{array}{llll}\text { C } & 0.249010194 & -2.943969868 & -0.074692325\end{array}$

$\begin{array}{llll}\mathrm{H} & 0.449428677 & -5.085749941 & 0.035303239\end{array}$

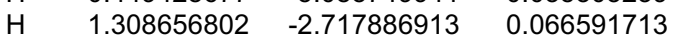

$\mathrm{Ni} \quad 0.000000000 \quad 0.000000000 \quad 0.000000000$

$\begin{array}{llll}\text { C } & 0.000000000 & 0.000000000 & 1.941270076\end{array}$

$\begin{array}{llll}\text { O } & 0.836868028 & 1.835220159 & -0.459862035\end{array}$

$\begin{array}{llll}\text { C } & 1.977186221 & 1.263314296 & -0.559828537\end{array}$

$\begin{array}{llll}\text { O } & 2.069773772 & 0.000000000 & -0.379413077\end{array}$

$\begin{array}{llll}\text { C } & 3.209901395 & 2.084526129 & -0.855420462\end{array}$

$\begin{array}{llll}\mathrm{H} & 2.957051335 & 2.951952712 & -1.480186754\end{array}$

$\begin{array}{llll}\mathrm{H} & 3.980962289 & 1.472020444 & -1.341478363\end{array}$

$\begin{array}{llll}\mathrm{H} & 3.622881992 & 2.464069701 & 0.093678092 \\ \mathrm{C} & 1.204997611 & -0.265392124 & 2.617918715\end{array}$

$\begin{array}{llll}\text { C } & -1.153541560 & 0.231472608 & 2.709904277\end{array}$

$\begin{array}{llll}\text { C } & -1.153541560 & 0.231472608 & 2.709904277 \\ \text { C } & -1.104433709 & 0.219800043 & 4.113437424\end{array}$

C $\quad 0.102407966 \quad-0.036987929 \quad 4.774486362$

C $\quad 1.257347547 \quad-0.280109218 \quad 4.021031951$

$\begin{array}{llll}\text { H } & 2.120750551 & -0.448127948 & 2.047164672\end{array}$

$\begin{array}{llll}\mathrm{H} & 2.209233659 & -0.482775928 & 4.524868671\end{array}$

$\begin{array}{llll}\mathrm{H} & 0.142543927 & -0.048147070 & 5.868267772\end{array}$

$\begin{array}{llll}\mathrm{H} & -2.114785794 & 0.434005254 & 2.225455631\end{array}$

$\begin{array}{llll}\mathrm{H} & -2.016591837 & 0.412088491 & 4.689700490\end{array}$ 


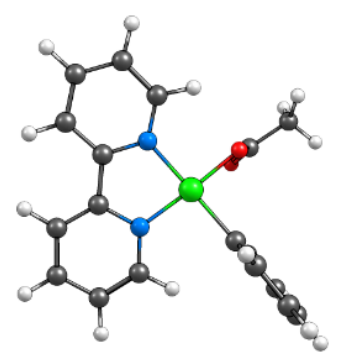

39

Aryl Carboxylate RKS $2.0 \AA \mathrm{Ni}-\mathrm{C}$ distance

$\begin{array}{lrrr}6 & -4.693690000 & -0.377607000 & 0.208141000 \\ 6 & -3.854927000 & -1.487845000 & 0.085423000 \\ 6 & -4.122191000 & 0.900456000 & 0.268838000 \\ 6 & -2.735425000 & 1.026280000 & 0.197011000 \\ 7 & -1.919905000 & -0.042136000 & 0.065034000 \\ 6 & -2.469004000 & -1.294077000 & 0.018640000 \\ 6 & -1.454282000 & -2.346522000 & -0.092158000 \\ 7 & -0.159670000 & -1.864447000 & -0.063635000 \\ 6 & -1.732600000 & -3.712503000 & -0.211972000 \\ 1 & -4.275847000 & -2.494799000 & 0.047034000 \\ 1 & -5.777362000 & -0.508728000 & 0.260458000 \\ 1 & -4.741255000 & 1.794336000 & 0.371448000 \\ 1 & -2.216058000 & 1.985699000 & 0.246974000 \\ 6 & -0.685062000 & -4.629431000 & -0.309868000 \\ 1 & -2.769328000 & -4.054693000 & -0.236214000 \\ 6 & 0.626916000 & -4.141422000 & -0.285373000 \\ 1 & -0.888556000 & -5.698538000 & -0.406233000 \\ 6 & 0.848774000 & -2.771420000 & -0.165918000 \\ 1 & 1.485399000 & -4.812332000 & -0.360653000 \\ 1 & 1.853427000 & -2.350398000 & -0.145195000 \\ 28 & 0.000000000 & 0.000000000 & 0.000000000 \\ 6 & 2.017685000 & 0.000000000 & 0.084979000 \\ 8 & 0.052094000 & 1.865249000 & 0.070135000 \\ 6 & 0.261577000 & 2.450291000 & -1.097058000 \\ 8 & 0.329276000 & 1.872299000 & -2.184483000 \\ 6 & 0.449054000 & 3.961064000 & -0.977444000 \\ 1 & -0.018794000 & 4.370609000 & -0.071441000 \\ 1 & 0.054955000 & 4.459146000 & -1.874048000 \\ 1 & 1.529372000 & 4.173332000 & -0.922681000 \\ 6 & 2.806700000 & 0.141022000 & -1.068122000 \\ 6 & 2.648874000 & -0.161636000 & 1.329587000 \\ 6 & 4.050421000 & -0.200158000 & 1.420206000 \\ 6 & 4.833231000 & -0.076418000 & 0.265035000 \\ 6 & 4.208332000 & 0.097402000 & -0.977005000 \\ 1 & 2.325914000 & 0.312631000 & -2.034689000 \\ 1 & 4.812917000 & 0.209702000 & -1.883759000 \\ 1 & 5.925280000 & -0.105543000 & 0.333930000 \\ 1 & 2.055300000 & -0.253714000 & 2.246171000 \\ 1 & 4.529567000 & -0.322386000 & 2.397970000\end{array}$

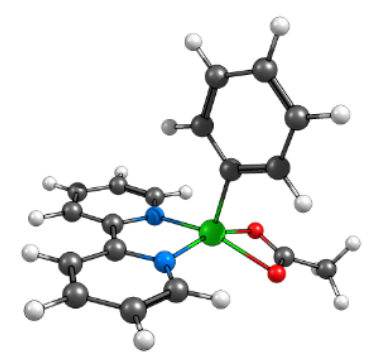

39

Aryl Carboxylate Triplet 2.0 Å Ni-C distance

$\begin{array}{lrrr}\mathrm{C} & 4.652962138 & 0.247672215 & -0.960748872 \\ \mathrm{C} & 3.858692324 & 1.385132560 & -0.827625658 \\ \mathrm{C} & 4.057100421 & -1.021601326 & -0.843960973 \\ \mathrm{~N} & 2.689729649 & -1.099243568 & -0.598133452 \\ \mathrm{C} & 1.912595075 & 0.000000000 & -0.473178628 \\ \mathrm{C} & 1.481802482 & 1.243488749 & -0.582375541 \\ \mathrm{~N} & 0.229634267 & 2.345598338 & -0.419358073 \\ \mathrm{C} & 1.853706499 & 3.712207247 & -0.239382774 \\ \mathrm{H} & 4.303699342 & 2.378801972 & -0.427210765 \\ \mathrm{H} & 5.724444066 & 0.343521623 & -1.153633587 \\ \mathrm{H} & 4.645958714 & -1.936104285 & -0.943246646 \\ \mathrm{H} & 2.161989463 & -2.051349421 & -0.498170852 \\ \mathrm{C} & 0.846356613 & 4.661432164 & -0.267123269 \\ \mathrm{H} & 2.891320837 & 4.029240368 & -0.554737258 \\ \mathrm{C} & -0.479645928 & 4.226007569 & -0.091062692 \\ \mathrm{H} & 1.086525305 & 5.727512033 & -0.273378287 \\ \mathrm{C} & -0.740747906 & 2.860136941 & -0.075816723 \\ \mathrm{H} & -1.298583365 & 4.937797875 & 0.033908133 \\ \mathrm{H} & -1.747396249 & 2.459066739 & 0.064654992 \\ \mathrm{Ni} & 0.000000000 & 0.000000000 & 0.000000000 \\ \mathrm{C} & 0.000000000 & 0.000000000 & 2.025833398 \\ \mathrm{O} & -0.516235484 & -1.949939411 & -0.459210797 \\ \mathrm{C} & -1.736503645 & -1.578212110 & -0.559195131 \\ \mathrm{O} & -2.040268981 & -0.348344554 & -0.379327501 \\ \mathrm{C} & -2.813835266 & -2.594882492 & -0.854191895 \\ \mathrm{H} & -2.419601513 & -3.407396504 & -1.479472468 \\ \mathrm{H} & -3.677155262 & -2.120378922 & -1.339275366 \\ \mathrm{H} & -3.156237996 & -3.038468724 & 0.095266302 \\ \mathrm{C} & -1.232470985 & 0.061798980 & 2.702298513 \\ \mathrm{C} & 1.175882678 & -0.036629647 & 2.794683615 \\ \mathrm{C} & 1.125319354 & -0.033305245 & 4.198219072 \\ \mathrm{C} & -0.107531611 & 0.019487328 & 4.859078134 \\ \mathrm{C} & -1.286691340 & 0.067585931 & 4.105440848 \\ \mathrm{H} & -2.165779319 & 0.090001930 & 2.131454940 \\ \mathrm{H} & -2.259113953 & 0.109418148 & 4.609077483 \\ \mathrm{H} & -0.149116328 & 0.023721355 & 5.952855194 \\ \mathrm{H} & 2.157434700 & -0.076313461 & 2.310253531 \\ \mathrm{H} & 2.056707289 & -0.071442599 & 4.774543371\end{array}$




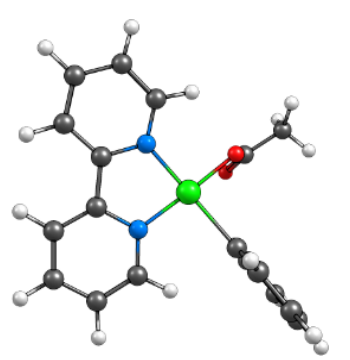

39

Aryl Carboxylate RKS $2.2 \AA \mathrm{Ni}-\mathrm{C}$ distance

$\begin{array}{lrrr}6 & -4.667690000 & -0.340216000 & 0.399001000 \\ 6 & -3.834751000 & -1.460232000 & 0.340554000 \\ 6 & -4.093105000 & 0.936002000 & 0.325213000 \\ 6 & -2.710527000 & 1.050861000 & 0.188883000 \\ 7 & -1.900876000 & -0.029012000 & 0.118851000 \\ 6 & -2.452735000 & -1.279613000 & 0.203189000 \\ 6 & -1.443466000 & -2.340459000 & 0.136965000 \\ 7 & -0.150389000 & -1.857802000 & 0.075468000 \\ 6 & -1.721836000 & -3.711431000 & 0.131440000 \\ 1 & -4.256663000 & -2.465399000 & 0.406745000 \\ 1 & -5.748464000 & -0.461773000 & 0.505220000 \\ 1 & -4.707209000 & 1.837899000 & 0.373320000 \\ 1 & -2.188234000 & 2.008557000 & 0.135640000 \\ 6 & -0.675153000 & -4.631715000 & 0.054800000 \\ 1 & -2.757221000 & -4.055577000 & 0.177954000 \\ 6 & 0.634777000 & -4.142788000 & -0.017480000 \\ 1 & -0.878170000 & -5.705228000 & 0.047430000 \\ 6 & 0.857419000 & -2.767490000 & -0.007486000 \\ 1 & 1.491064000 & -4.817459000 & -0.083340000 \\ 1 & 1.859289000 & -2.342582000 & -0.060260000 \\ 28 & 0.000000000 & 0.000000000 & 0.000000000 \\ 6 & 2.210107000 & 0.000000000 & 0.068146000 \\ 8 & 0.074219000 & 1.860972000 & -0.064346000 \\ 6 & 0.332518000 & 2.353160000 & -1.264029000 \\ 8 & 0.422543000 & 1.692127000 & -2.301484000 \\ 6 & 0.550808000 & 3.864041000 & -1.250899000 \\ 1 & 0.037046000 & 4.353341000 & -0.411606000 \\ 1 & 0.226165000 & 4.297101000 & -2.206939000 \\ 1 & 1.630313000 & 4.058354000 & -1.141099000 \\ 6 & 2.971220000 & 0.113324000 & -1.102453000 \\ 6 & 2.850501000 & -0.117764000 & 1.308629000 \\ 6 & 4.254430000 & -0.140120000 & 1.379426000 \\ 6 & 5.017579000 & -0.042891000 & 0.208673000 \\ 6 & 4.375204000 & 0.086342000 & -1.029904000 \\ 1 & 2.471408000 & 0.249395000 & -2.064842000 \\ 1 & 4.967101000 & 0.176188000 & -1.947554000 \\ 1 & 6.110802000 & -0.058803000 & 0.262541000 \\ 1 & 2.268332000 & -0.188293000 & 2.234054000 \\ 1 & 4.750248000 & -0.228379000 & 2.352605000 \\ & & \end{array}$

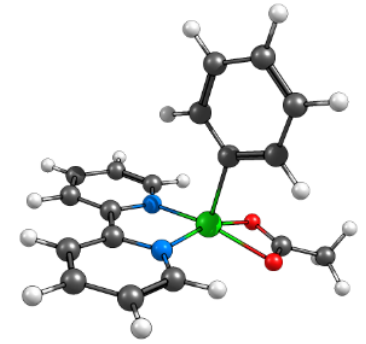

39

Aryl Carboxylate Triplet 2.2 $\AA \mathrm{Ni}-\mathrm{C}$ distance

$\begin{array}{lrrr}\mathrm{C} & 4.692842251 & 0.243448950 & -0.673192936 \\ \mathrm{C} & 3.893960871 & 1.382385145 & -0.574593034 \\ \mathrm{C} & 4.088687563 & -1.023608091 & -0.602009839 \\ \mathrm{C} & 2.708182227 & -1.099817676 & -0.438355225 \\ \mathrm{~N} & 1.927429704 & 0.000000000 & -0.348712378 \\ \mathrm{C} & 2.506212156 & 1.241182483 & -0.409963344 \\ \mathrm{C} & 1.545042921 & 2.346652362 & -0.281769430 \\ \mathrm{~N} & 0.243903154 & 1.932936002 & -0.152741106 \\ \mathrm{C} & 1.875141420 & 3.711355013 & -0.268997848 \\ \mathrm{H} & 4.345938815 & 2.375557190 & -0.624960397 \\ \mathrm{H} & 5.773901983 & 0.338615433 & -0.802325139 \\ \mathrm{H} & 4.680267902 & -1.938973987 & -0.673731656 \\ \mathrm{H} & 2.173993051 & -2.051576885 & -0.377266195 \\ \mathrm{C} & 0.863328261 & 4.661494134 & -0.135677401 \\ \mathrm{H} & 2.916723004 & 4.027975884 & -0.359574183 \\ \mathrm{C} & -0.466899457 & 4.226694283 & -0.009003596 \\ \mathrm{H} & 1.105200201 & 5.727152781 & -0.125261999 \\ \mathrm{C} & -0.730523809 & 2.860236717 & -0.017602335 \\ \mathrm{H} & -1.288570248 & 4.938579947 & 0.096296870 \\ \mathrm{H} & -1.741833015 & 2.458825699 & 0.083293418 \\ \mathrm{Ni} & 0.000000000 & 0.000000000 & 0.000000000 \\ \mathrm{C} & 0.000000000 & 0.000000000 & 2.197500429 \\ \mathrm{O} & -0.536904347 & -1.943675784 & -0.430091582 \\ \mathrm{C} & -1.758921921 & -1.566314538 & -0.455967846 \\ \mathrm{O} & -2.041940901 & -0.331157593 & -0.272071153 \\ \mathrm{C} & -2.859809208 & -2.578310207 & -0.666047091 \\ \mathrm{H} & -2.522979478 & -3.384568968 & -1.331940944 \\ \mathrm{H} & -3.761474175 & -2.098401716 & -1.068734024 \\ \mathrm{H} & -3.116671995 & -3.032993284 & 0.304858135 \\ \mathrm{C} & -1.238215885 & 0.057306671 & 2.849842482 \\ \mathrm{C} & 1.174446651 & -0.031449408 & 2.957346771 \\ \mathrm{C} & 1.115457604 & -0.018213462 & 4.361872019 \\ \mathrm{C} & -0.123663664 & 0.034419658 & 5.012111005 \\ \mathrm{C} & -1.300896379 & 0.071949651 & 4.253877591 \\ \mathrm{H} & -2.164770989 & 0.079591638 & 2.267185310 \\ \mathrm{H} & -2.274962260 & 0.112345414 & 4.754810681 \\ \mathrm{H} & -0.171681095 & 0.045570898 & 6.105699091 \\ \mathrm{H} & 2.156121867 & -0.073288968 & 2.472046127 \\ \mathrm{H} & 2.041782018 & -0.049648609 & 4.947045036\end{array}$




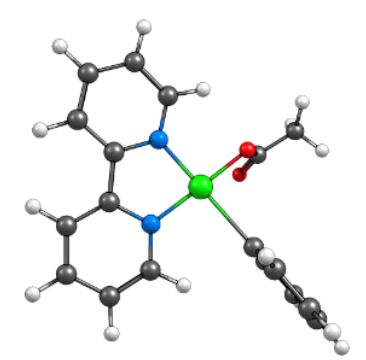

39

Aryl Carboxylate RKS 2.4 A Ni-C distance

$\begin{array}{lrrr}6 & -4.669212000 & -0.337664000 & 0.196995000 \\ 6 & -3.832856000 & -1.456448000 & 0.227003000 \\ 6 & -4.093614000 & 0.935805000 & 0.087977000 \\ 6 & -2.706864000 & 1.049996000 & 0.006576000 \\ 7 & -1.893561000 & -0.030042000 & 0.023002000 \\ 6 & -2.446630000 & -1.278690000 & 0.141548000 \\ 6 & -1.433325000 & -2.337617000 & 0.162176000 \\ 7 & -0.141112000 & -1.850691000 & 0.135718000 \\ 6 & -1.704594000 & -3.709439000 & 0.196183000 \\ 1 & -4.255532000 & -2.459105000 & 0.321502000 \\ 1 & -5.753365000 & -0.457696000 & 0.261950000 \\ 1 & -4.710693000 & 1.836751000 & 0.066825000 \\ 1 & -2.183616000 & 2.005795000 & -0.068698000 \\ 6 & -0.650147000 & -4.624377000 & 0.194686000 \\ 1 & -2.739135000 & -4.058950000 & 0.213248000 \\ 6 & 0.659410000 & -4.130649000 & 0.154167000 \\ 1 & -0.847107000 & -5.698782000 & 0.219029000 \\ 6 & 0.875484000 & -2.754353000 & 0.123031000 \\ 1 & 1.520483000 & -4.802344000 & 0.145354000 \\ 1 & 1.875031000 & -2.321633000 & 0.094835000 \\ 28 & 0.000000000 & 0.000000000 & 0.000000000 \\ 6 & 2.391834000 & 0.000000000 & 0.229742000 \\ 8 & 0.092403000 & 1.854557000 & -0.125019000 \\ 6 & 0.483230000 & 2.288894000 & -1.310912000 \\ 8 & 0.665197000 & 1.577545000 & -2.302402000 \\ 6 & 0.723712000 & 3.795429000 & -1.343664000 \\ 1 & 0.116586000 & 4.331241000 & -0.600578000 \\ 1 & 0.528445000 & 4.183464000 & -2.352641000 \\ 1 & 1.783759000 & 3.983661000 & -1.106960000 \\ 6 & 3.205034000 & 0.103799000 & -0.902325000 \\ 6 & 2.956788000 & -0.097039000 & 1.504587000 \\ 6 & 4.356100000 & -0.105794000 & 1.650303000 \\ 6 & 5.178791000 & -0.015907000 & 0.520046000 \\ 6 & 4.604057000 & 0.090098000 & -0.753643000 \\ 1 & 2.753735000 & 0.217064000 & -1.890967000 \\ 1 & 5.244262000 & 0.170701000 & -1.639257000 \\ 1 & 6.267670000 & -0.021476000 & 0.632613000 \\ 1 & 2.324283000 & -0.161514000 & 2.396650000 \\ 1 & 4.800214000 & -0.177361000 & 2.649599000 \\ & & \end{array}$

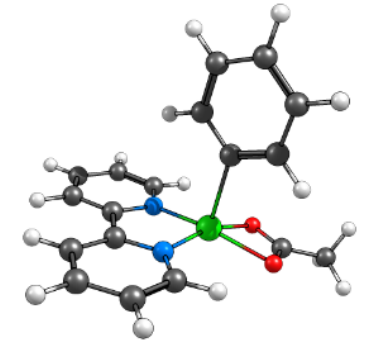

39

Aryl Carboxylate Triplet 2.4 A Ni-C distance

$\begin{array}{lrrr}\mathrm{C} & 4.705912086 & 0.243429899 & -0.532806844 \\ \mathrm{C} & 3.904978049 & 1.382433130 & -0.443768400 \\ \mathrm{C} & 4.099339420 & -1.022842819 & -0.489291360 \\ \mathrm{C} & 2.714416756 & -1.099405743 & -0.363531767 \\ \mathrm{~N} & 1.932270860 & 0.000000000 & -0.282900696 \\ \mathrm{C} & 2.514161271 & 1.240425754 & -0.315961048 \\ \mathrm{C} & 1.550360583 & 2.346604217 & -0.196454391 \\ \mathrm{~N} & 0.248057265 & 1.931468823 & -0.092422757 \\ \mathrm{C} & 1.880954620 & 3.710196364 & -0.166436126 \\ \mathrm{H} & 4.358728981 & 2.375669463 & -0.471718960 \\ \mathrm{H} & 5.790053046 & 0.338926339 & -0.632385365 \\ \mathrm{H} & 4.691922813 & -1.938165213 & -0.553225397 \\ \mathrm{H} & 2.178527664 & -2.051396355 & -0.325731645 \\ \mathrm{C} & 0.867026105 & 4.659738637 & -0.038592697 \\ \mathrm{H} & 2.923737043 & 4.027383520 & -0.239350423 \\ \mathrm{C} & -0.464208569 & 4.224095195 & 0.064057138 \\ \mathrm{H} & 1.108937233 & 5.725152221 & -0.014117777 \\ \mathrm{C} & -0.728217100 & 2.857462688 & 0.036243295 \\ \mathrm{H} & -1.287163353 & 4.935042448 & 0.165853949 \\ \mathrm{H} & -1.740878634 & 2.454988225 & 0.117565906 \\ \mathrm{Ni} & 0.000000000 & 0.000000000 & 0.000000000 \\ \mathrm{C} & 0.000000000 & 0.000000000 & 2.369167217 \\ \mathrm{O} & -0.548099175 & -1.939838356 & -0.410363773 \\ \mathrm{C} & -1.769502225 & -1.559543571 & -0.395139553 \\ \mathrm{O} & -2.041812984 & -0.322185199 & -0.208296562 \\ \mathrm{C} & -2.879745113 & -2.569431078 & -0.559774334 \\ \mathrm{H} & -2.570848400 & -3.377414376 & -1.237078053 \\ \mathrm{H} & -3.795720107 & -2.088569473 & -0.927445310 \\ \mathrm{H} & -3.098639536 & -3.022265390 & 0.421197000 \\ \mathrm{C} & -1.243583104 & 0.052514568 & 3.004505843 \\ \mathrm{C} & 1.172430745 & -0.028760456 & 3.126433206 \\ \mathrm{C} & 1.104443106 & -0.013021677 & 4.531426115 \\ \mathrm{C} & -0.139834900 & 0.036816220 & 5.172691256 \\ \mathrm{C} & -1.314056744 & 0.069185674 & 4.408990596 \\ \mathrm{H} & -2.165268990 & 0.071853329 & 2.413668786 \\ \mathrm{H} & -2.290563897 & 0.106886388 & 4.905575603 \\ \mathrm{H} & -0.194313321 & 0.049388008 & 6.265984983 \\ \mathrm{H} & 2.155411989 & -0.068976919 & 2.643072968 \\ \mathrm{H} & 2.026649227 & -0.040759697 & 5.123450598\end{array}$




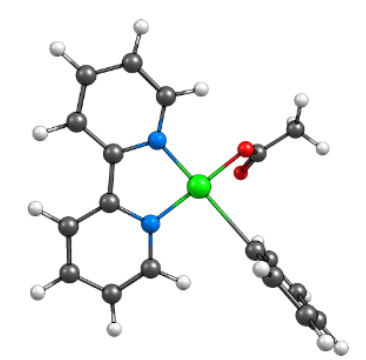

39

Aryl Carboxylate RKS 2.6 A Ni-C distance

$\begin{array}{lrrr}6 & -4.650982000 & -0.407914000 & 0.330148000 \\ 6 & -3.801992000 & -1.515781000 & 0.265619000 \\ 6 & -4.093534000 & 0.877183000 & 0.273942000 \\ 6 & -2.712136000 & 1.014349000 & 0.149994000 \\ 7 & -1.887326000 & -0.054819000 & 0.074642000 \\ 6 & -2.421979000 & -1.316088000 & 0.140046000 \\ 6 & -1.396756000 & -2.361123000 & 0.061803000 \\ 7 & -0.112354000 & -1.855567000 & 0.025374000 \\ 6 & -1.648968000 & -3.736239000 & 0.017867000 \\ 1 & -4.209724000 & -2.527661000 & 0.318729000 \\ 1 & -5.730593000 & -0.545373000 & 0.427597000 \\ 1 & -4.720863000 & 1.769731000 & 0.326659000 \\ 1 & -2.202225000 & 1.979540000 & 0.111809000 \\ 6 & -0.582685000 & -4.632779000 & -0.076133000 \\ 1 & -2.677631000 & -4.102054000 & 0.046260000 \\ 6 & 0.718589000 & -4.118236000 & -0.128833000 \\ 1 & -0.764593000 & -5.709476000 & -0.112974000 \\ 6 & 0.916090000 & -2.739606000 & -0.078981000 \\ 1 & 1.587192000 & -4.775344000 & -0.208724000 \\ 1 & 1.907678000 & -2.288387000 & -0.110176000 \\ 28 & 0.000000000 & 0.000000000 & 0.000000000 \\ 6 & 2.586202000 & 0.000000000 & 0.207671000 \\ 8 & 0.078132000 & 1.858565000 & -0.014305000 \\ 6 & 0.487561000 & 2.352695000 & -1.169975000 \\ 8 & 0.697856000 & 1.687613000 & -2.187961000 \\ 6 & 0.722238000 & 3.859301000 & -1.125697000 \\ 1 & 0.083792000 & 4.358747000 & -0.383432000 \\ 1 & 0.563921000 & 4.291909000 & -2.122855000 \\ 1 & 1.771047000 & 4.040271000 & -0.838729000 \\ 6 & 3.363817000 & 0.259947000 & -0.922042000 \\ 6 & 3.179480000 & -0.244047000 & 1.447356000 \\ 6 & 4.582611000 & -0.245524000 & 1.557775000 \\ 6 & 5.374295000 & 0.000008000 & 0.428347000 \\ 6 & 4.766601000 & 0.253680000 & -0.808757000 \\ 1 & 2.884231000 & 0.485808000 & -1.877906000 \\ 1 & 5.383020000 & 0.455729000 & -1.691979000 \\ 1 & 6.465617000 & 0.000474000 & 0.513975000 \\ 1 & 2.569650000 & -0.429853000 & 2.338076000 \\ 1 & 5.053144000 & -0.433244000 & 2.529639000 \\ & & \end{array}$

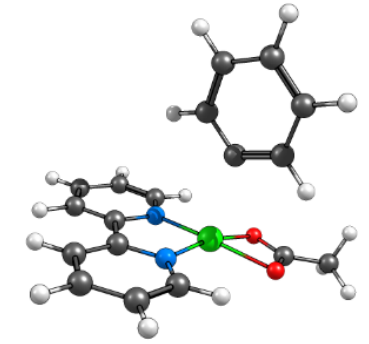

39

Aryl Carboxylate Triplet 2.7 $\AA \mathrm{Ni}-\mathrm{C}$ distance

$\begin{array}{lrrr}\mathrm{C} & 4.693846108 & 0.253460832 & -0.554366775 \\ \mathrm{C} & 3.894627874 & 1.389045429 & -0.406680804 \\ \mathrm{C} & 4.089200320 & -1.012810663 & -0.530819618 \\ \mathrm{C} & 2.707899416 & -1.094912239 & -0.366321697 \\ \mathrm{~N} & 1.928311319 & 0.000000000 & -0.221828487 \\ \mathrm{C} & 2.510556160 & 1.240647729 & -0.235761798 \\ \mathrm{C} & 1.548056697 & 2.342202156 & -0.053801320 \\ \mathrm{~N} & 0.247098920 & 1.920279003 & 0.036678933 \\ \mathrm{C} & 1.879225845 & 3.701559864 & 0.035714322 \\ \mathrm{H} & 4.345658143 & 2.383799227 & -0.425501266 \\ \mathrm{H} & 5.774062049 & 0.353410905 & -0.686665104 \\ \mathrm{H} & 4.679710342 & -1.925370431 & -0.639115323 \\ \mathrm{H} & 2.173835304 & -2.048409509 & -0.344781612 \\ \mathrm{C} & 0.865655277 & 4.644460143 & 0.217130977 \\ \mathrm{H} & 2.921709780 & 4.021606183 & -0.029062563 \\ \mathrm{C} & -0.463319506 & 4.203240627 & 0.310064103 \\ \mathrm{H} & 1.107569122 & 5.707593343 & 0.290509146 \\ \mathrm{C} & -0.728071917 & 2.838946343 & 0.217630101 \\ \mathrm{H} & -1.285603551 & 4.907746328 & 0.453960205 \\ \mathrm{H} & -1.739985656 & 2.432514233 & 0.286871132 \\ \mathrm{Ni} & 0.000000000 & 0.000000000 & 0.00000000 \\ \mathrm{C} & 0.000000000 & 0.000000000 & 2.712499828 \\ \mathrm{O} & -0.562161219 & -1.930162793 & -0.379252813 \\ \mathrm{C} & -1.781587404 & -1.554084277 & -0.278133807 \\ \mathrm{O} & -2.042874622 & -0.317621379 & -0.072952058 \\ \mathrm{C} & -2.896421410 & -2.568344358 & -0.364656728 \\ \mathrm{H} & -2.637305805 & -3.368887967 & -1.071146270 \\ \mathrm{H} & -3.840357075 & -2.089350958 & -0.655952928 \\ \mathrm{H} & -3.035958629 & -3.031091487 & 0.626063913 \\ \mathrm{C} & -1.260563817 & 0.039697057 & 3.303476211 \\ \mathrm{C} & 1.160266088 & -0.025364584 & 3.480455571 \\ \mathrm{C} & 1.058358005 & -0.011459771 & 4.884649038 \\ \mathrm{C} & -0.202238258 & 0.028724381 & 5.495206525 \\ \mathrm{C} & -1.361178330 & 0.053740684 & 4.707385664 \\ \mathrm{H} & -2.166176973 & 0.053919817 & 2.687670735 \\ \mathrm{H} & -2.348001623 & 0.082647960 & 5.183926839 \\ \mathrm{H} & -0.281405115 & 0.038742472 & 6.586961699 \\ \mathrm{H} & 2.151207571 & -0.058982002 & 3.013027861 \\ \mathrm{H} & 1.965965116 & -0.033632154 & 5.499173690\end{array}$




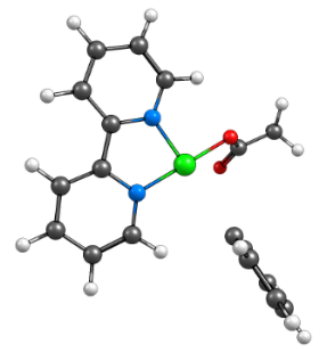

39

Aryl Carboxylate RKS $2.9 \AA$ Ni-C distance

$\begin{array}{lrrr}6 & 4.666210000 & 0.280284000 & -0.003624000 \\ 6 & 3.845413000 & 1.410286000 & -0.045116000 \\ 6 & 4.071357000 & -0.990225000 & -0.001114000 \\ 6 & 2.682488000 & -1.091459000 & -0.043028000 \\ 7 & 1.883612000 & 0.000000000 & -0.096503000 \\ 6 & 2.455506000 & 1.248249000 & -0.091074000 \\ 6 & 1.455926000 & 2.319683000 & -0.144812000 \\ 7 & 0.160594000 & 1.850792000 & -0.059783000 \\ 6 & 1.735042000 & 3.683916000 & -0.280374000 \\ 1 & 4.282444000 & 2.411416000 & -0.032863000 \\ 1 & 5.752888000 & 0.388692000 & 0.031889000 \\ 1 & 4.676983000 & -1.898602000 & 0.034164000 \\ 1 & 2.145813000 & -2.042948000 & -0.030428000 \\ 6 & 0.683962000 & 4.600716000 & -0.349135000 \\ 1 & 2.770914000 & 4.024010000 & -0.346598000 \\ 6 & -0.630492000 & 4.119151000 & -0.289377000 \\ 1 & 0.886689000 & 5.669108000 & -0.455166000 \\ 6 & -0.854898000 & 2.751194000 & -0.148834000 \\ 1 & -1.486598000 & 4.794621000 & -0.347873000 \\ 1 & -1.854756000 & 2.321769000 & -0.079978000 \\ 28 & 0.000000000 & 0.000000000 & 0.000000000 \\ 6 & -2.791804000 & 0.202148000 & 0.810161000 \\ 8 & -0.163798000 & -1.861240000 & 0.060620000 \\ 6 & -0.893088000 & -2.264828000 & -0.960505000 \\ 8 & -1.315485000 & -1.508715000 & -1.845182000 \\ 6 & -1.216127000 & -3.752148000 & -0.940863000 \\ 1 & -0.404802000 & -4.343560000 & -0.493181000 \\ 1 & -1.429859000 & -4.104199000 & -1.958792000 \\ 1 & -2.118023000 & -3.908451000 & -0.326584000 \\ 6 & -3.768013000 & -0.126845000 & -0.126357000 \\ 6 & -3.116466000 & 0.607300000 & 2.101567000 \\ 6 & -4.473462000 & 0.701657000 & 2.467215000 \\ 6 & -5.472624000 & 0.386515000 & 1.536940000 \\ 6 & -5.123217000 & -0.027045000 & 0.244177000 \\ 1 & -3.483663000 & -0.468756000 & -1.125062000 \\ 1 & -5.904182000 & -0.280699000 & -0.481687000 \\ 1 & -6.526980000 & 0.458239000 & 1.822330000 \\ 1 & -2.339755000 & 0.847808000 & 2.835293000 \\ 1 & -4.744146000 & 1.017245000 & 3.481369000 \\ & & \end{array}$

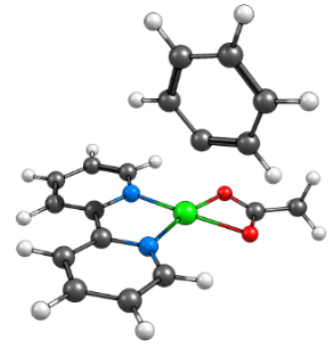

39

Aryl Carboxylate Triplet 2.9 $\AA \mathrm{Ni}-\mathrm{C}$ distance

$\begin{array}{lrrr}\mathrm{C} & 4.708041113 & 0.273931660 & -0.098425746 \\ \mathrm{C} & 3.891006879 & 1.405125617 & -0.037857388 \\ \mathrm{C} & 4.113807696 & -0.997497697 & -0.060888739 \\ \mathrm{C} & 2.726488977 & -1.090200246 & 0.030970616 \\ \mathrm{~N} & 1.929074581 & 0.000000000 & 0.106257614 \\ \mathrm{C} & 2.502416755 & 1.246809062 & 0.071940062 \\ \mathrm{C} & 1.519870742 & 2.338251388 & 0.161730674 \\ \mathrm{~N} & 0.222899102 & 1.909675908 & 0.026968357 \\ \mathrm{C} & 1.822419219 & 3.689649516 & 0.376537447 \\ \mathrm{H} & 4.328687279 & 2.404907755 & -0.089903473 \\ \mathrm{H} & 5.792471500 & 0.380621473 & -0.181873970 \\ \mathrm{H} & 4.717890851 & -1.906799994 & -0.100010179 \\ \mathrm{H} & 2.201686736 & -2.048734644 & 0.054150126 \\ \mathrm{C} & 0.785782713 & 4.623034985 & 0.438527564 \\ \mathrm{H} & 2.859271871 & 4.006438448 & 0.511778993 \\ \mathrm{C} & -0.537925845 & 4.177251443 & 0.296416270 \\ \mathrm{H} & 1.003774662 & 5.680497809 & 0.606910791 \\ \mathrm{C} & -0.775970629 & 2.818898859 & 0.103151370 \\ \mathrm{H} & -1.377716895 & 4.874518527 & 0.337971709 \\ \mathrm{H} & -1.783537045 & 2.409801850 & -0.002010183 \\ \mathrm{Ni} & 0.000000000 & 0.000000000 & 0.000000000 \\ \mathrm{C} & -0.555032749 & 0.170577451 & 2.825112220 \\ \mathrm{O} & -0.486998438 & -1.939189324 & -0.332493792 \\ \mathrm{C} & -1.715917447 & -1.583732965 & -0.410250161 \\ \mathrm{O} & -2.013441084 & -0.342798596 & -0.316840067 \\ \mathrm{C} & -2.796047587 & -2.624592073 & -0.572186231 \\ \mathrm{H} & -2.437849693 & -3.462764235 & -1.185590268 \\ \mathrm{H} & -3.700336153 & -2.184850000 & -1.012865558 \\ \mathrm{H} & -3.056458522 & -3.025678787 & 0.421008082 \\ \mathrm{C} & -1.910528960 & 0.230468112 & 3.128456223 \\ \mathrm{C} & 0.426574474 & 0.203413500 & 3.807886826 \\ \mathrm{C} & 0.033540049 & 0.303608631 & 5.156830732 \\ \mathrm{C} & -1.326691508 & 0.367684499 & 5.487963572 \\ \mathrm{C} & -2.298535592 & 0.330643026 & 4.478601091 \\ \mathrm{H} & -2.666260717 & 0.194662096 & 2.336741263 \\ \mathrm{H} & -3.362432114 & 0.377333642 & 4.738487376 \\ \mathrm{H} & -1.630334943 & 0.444548095 & 6.536771882 \\ \mathrm{H} & 1.491328250 & 0.150192609 & 3.554823550 \\ \mathrm{H} & 0.793460341 & 0.329628278 & 5.946468854\end{array}$




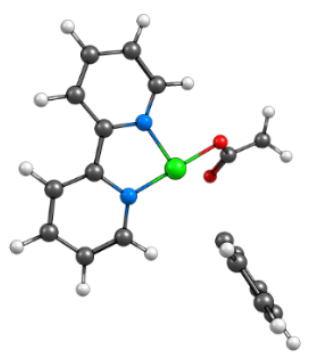

39

Aryl Carboxylate RKS 3.1 $\AA$ Ni-C distance

$\begin{array}{lrrr}6 & -4.619758000 & 0.605388000 & -0.117922000 \\ 6 & -4.031447000 & -0.655821000 & -0.188015000 \\ 6 & -3.789592000 & 1.745195000 & -0.096700000 \\ 6 & -2.411105000 & 1.579244000 & -0.148861000 \\ 7 & -1.827577000 & 0.358106000 & -0.255352000 \\ 6 & -2.634007000 & -0.767745000 & -0.260532000 \\ 6 & -1.863336000 & -1.998532000 & -0.333645000 \\ 7 & -0.518763000 & -1.794315000 & -0.081858000 \\ 6 & -2.355936000 & -3.282758000 & -0.618016000 \\ 1 & -4.648607000 & -1.557591000 & -0.162650000 \\ 1 & -5.706371000 & 0.706564000 & -0.062780000 \\ 1 & -4.211344000 & 2.751591000 & -0.047859000 \\ 1 & -1.715576000 & 2.421738000 & -0.125777000 \\ 6 & -1.481886000 & -4.366584000 & -0.643339000 \\ 1 & -3.417237000 & -3.420415000 & -0.839597000 \\ 6 & -0.111892000 & -4.144608000 & -0.398234000 \\ 1 & -1.853037000 & -5.370817000 & -0.862167000 \\ 6 & 0.327118000 & -2.852330000 & -0.135333000 \\ 1 & 0.604914000 & -4.968463000 & -0.398996000 \\ 1 & 1.374753000 & -2.617468000 & 0.063810000 \\ 28 & 0.000000000 & 0.000000000 & 0.000000000 \\ 6 & 0.540181000 & -0.773187000 & 2.997753000 \\ 8 & 0.779251000 & 1.799592000 & 0.112648000 \\ 6 & 1.950554000 & 1.271857000 & 0.085397000 \\ 8 & 2.027061000 & 0.000000000 & -0.030295000 \\ 6 & 3.179691000 & 2.126783000 & 0.224800000 \\ 1 & 3.024041000 & 3.105668000 & -0.249101000 \\ 1 & 4.053711000 & 1.624522000 & -0.210124000 \\ 1 & 3.376993000 & 2.300537000 & 1.295258000 \\ 6 & 1.872979000 & -1.027322000 & 3.285811000 \\ 6 & -0.469986000 & -0.831069000 & 3.944907000 \\ 6 & -0.120624000 & -1.163142000 & 5.269002000 \\ 6 & 1.216674000 & -1.425857000 & 5.596723000 \\ 6 & 2.212446000 & -1.359427000 & 4.612522000 \\ 1 & 2.642589000 & -0.970185000 & 2.509473000 \\ 1 & 3.257034000 & -1.565212000 & 4.872565000 \\ 1 & 1.484485000 & -1.683713000 & 6.626008000 \\ 1 & -1.514384000 & -0.625078000 & 3.686752000 \\ 1 & -0.896633000 & -1.215647000 & 6.041076000 \\ & & \end{array}$

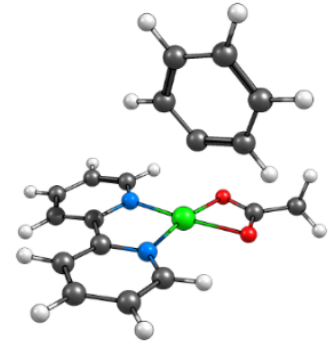

39

Aryl Carboxylate Triplet 3.1 $\AA$ Ni-C distance

$\begin{array}{lrrr}\mathrm{C} & 4.668911097 & 0.278251076 & -0.429837405 \\ \mathrm{C} & 3.858299803 & 1.408116680 & -0.302925651 \\ \mathrm{C} & 4.080818908 & -0.994433766 & -0.335491836 \\ \mathrm{C} & 2.707940989 & -1.089380945 & -0.121376855 \\ \mathrm{C} & 1.917119386 & 0.000000000 & 0.026456249 \\ \mathrm{C} & 2.485671567 & 1.249532558 & -0.066792678 \\ \mathrm{~N} & 1.511182616 & 2.333989366 & 0.099864848 \\ \mathrm{C} & 0.208817057 & 1.900299921 & 0.016192761 \\ \mathrm{H} & 1.817254965 & 3.681636413 & 0.333117036 \\ \mathrm{H} & 5.741194446 & 2.409057082 & -0.404644737 \\ \mathrm{H} & 4.680643299 & -1.903287410 & -0.422230115 \\ \mathrm{H} & 2.189551330 & -2.048901676 & -0.049368054 \\ \mathrm{C} & 0.781075129 & 4.607311878 & 0.468015400 \\ \mathrm{H} & 2.858637338 & 3.998291998 & 0.429577106 \\ \mathrm{C} & -0.546923935 & 4.156663945 & 0.382754841 \\ \mathrm{H} & 1.002011876 & 5.661647464 & 0.651477712 \\ \mathrm{C} & -0.788838137 & 2.802344362 & 0.170175671 \\ \mathrm{H} & -1.386793431 & 4.848106461 & 0.481052541 \\ \mathrm{H} & -1.798831417 & 2.390690178 & 0.106492804 \\ \mathrm{Ni} & 0.000000000 & 0.000000000 & 0.000000000 \\ \mathrm{C} & -0.494358589 & 0.204258580 & 3.008654402 \\ \mathrm{O} & -0.496911519 & -1.938756313 & -0.253165834 \\ \mathrm{C} & -1.729583400 & -1.588666604 & -0.238780212 \\ \mathrm{O} & -2.018169879 & -0.345251393 & -0.139118418 \\ \mathrm{C} & -2.817382683 & -2.630884557 & -0.302242405 \\ \mathrm{H} & -2.502908618 & -3.481620780 & -0.922173744 \\ \mathrm{H} & -3.750061797 & -2.198620291 & -0.687810204 \\ \mathrm{H} & -3.008515956 & -3.010862936 & 0.714699689 \\ \mathrm{C} & -1.847151113 & 0.254104628 & 3.315733664 \\ \mathrm{C} & 0.501541073 & 0.252216291 & 3.973282430 \\ \mathrm{C} & 0.119308393 & 0.359045062 & 5.325053451 \\ \mathrm{C} & -1.238693167 & 0.413309488 & 5.668064735 \\ \mathrm{C} & -2.221311587 & 0.360636224 & 4.669589742 \\ \mathrm{H} & -2.606812819 & 0.207140774 & 2.528624495 \\ \mathrm{H} & -3.282459534 & 0.400718981 & 4.940946701 \\ \mathrm{H} & -1.532455929 & 0.495002918 & 6.719177940 \\ \mathrm{H} & 1.562923661 & 0.206285415 & 3.706425883 \\ \mathrm{H} & 0.885521482 & 0.398109997 & 6.107812395\end{array}$




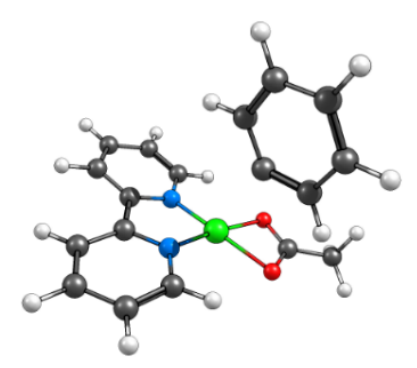

39

Aryl Carboxylate RKS $3.3 \AA \mathrm{Ni}-\mathrm{C}$ distance

$\begin{array}{lrrr}6 & -4.625000000 & 0.562765000 & 0.051717000 \\ 6 & -4.026528000 & -0.695595000 & 0.063932000 \\ 6 & -3.807704000 & 1.705940000 & -0.067091000 \\ 6 & -2.431214000 & 1.545359000 & -0.170083000 \\ 7 & -1.839731000 & 0.324054000 & -0.197669000 \\ 6 & -2.632969000 & -0.803844000 & -0.066449000 \\ 6 & -1.853321000 & -2.030858000 & -0.072076000 \\ 7 & -0.501013000 & -1.797194000 & 0.106991000 \\ 6 & -2.344375000 & -3.336801000 & -0.231943000 \\ 1 & -4.631521000 & -1.595976000 & 0.198370000 \\ 1 & -5.708851000 & 0.660698000 & 0.150401000 \\ 1 & -4.237910000 & 2.709737000 & -0.087204000 \\ 1 & -1.745011000 & 2.391666000 & -0.254276000 \\ 6 & -1.461853000 & -4.413931000 & -0.206554000 \\ 1 & -3.412391000 & -3.498337000 & -0.398728000 \\ 6 & -0.085037000 & -4.163832000 & -0.038981000 \\ 1 & -1.831920000 & -5.434896000 & -0.328310000 \\ 6 & 0.351847000 & -2.851583000 & 0.099761000 \\ 1 & 0.639190000 & -4.980452000 & -0.003364000 \\ 1 & 1.405133000 & -2.595135000 & 0.232760000 \\ 28 & 0.000000000 & 0.000000000 & 0.00000000 \\ 6 & 0.760439000 & -0.600966000 & 3.169206000 \\ 8 & 0.775545000 & 1.807903000 & -0.072512000 \\ 6 & 1.945155000 & 1.278084000 & -0.074996000 \\ 8 & 2.020293000 & 0.000000000 & -0.069412000 \\ 6 & 3.178260000 & 2.138763000 & -0.048674000 \\ 1 & 3.009298000 & 3.071037000 & -0.604739000 \\ 1 & 4.039255000 & 1.596688000 & -0.461291000 \\ 1 & 3.407188000 & 2.408010000 & 0.995416000 \\ 6 & 2.120674000 & -0.803808000 & 3.339963000 \\ 6 & -0.160225000 & -0.619634000 & 4.203261000 \\ 6 & 0.320216000 & -0.858469000 & 5.506518000 \\ 6 & 1.689377000 & -1.068597000 & 5.722295000 \\ 6 & 2.588986000 & -1.042346000 & 4.647757000 \\ 1 & 2.811113000 & -0.777914000 & 2.491364000 \\ 1 & 3.658361000 & -1.206794000 & 4.821627000 \\ 1 & 2.057994000 & -1.253754000 & 6.735843000 \\ 1 & -1.228890000 & -0.454642000 & 4.029779000 \\ 1 & -0.379286000 & -0.879596000 & 6.349722000\end{array}$

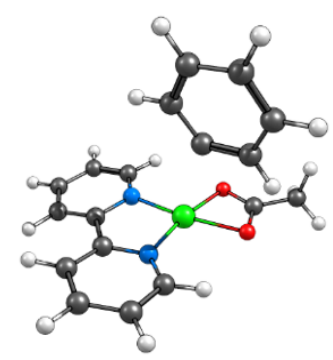

39

Aryl Carboxylate Triplet 3.3 $\AA \mathrm{Ni}-\mathrm{C}$ distance

$\begin{array}{lrrr}\mathrm{C} & 4.670889835 & 0.222555601 & -0.200245452 \\ \mathrm{C} & 3.864546315 & 1.361288400 & -0.230724850 \\ \mathrm{C} & 4.066648314 & -1.033067011 & -0.006783851 \\ \mathrm{~N} & 2.685193482 & -1.101961081 & 0.147290364 \\ \mathrm{C} & 1.895501881 & 0.000000000 & 0.149667621 \\ \mathrm{C} & 2.480413805 & 1.233892890 & -0.046532731 \\ \mathrm{~N} & 0.206805874 & 2.326019874 & -0.042924389 \\ \mathrm{C} & 1.798293215 & 1.880662566 & -0.176769225 \\ \mathrm{H} & 4.302145813 & 2.341091766 & 0.088091007 \\ \mathrm{H} & 5.751543759 & 0.306191878 & -0.417543605 \\ \mathrm{H} & 4.662250857 & -1.948048644 & 0.031174123 \\ \mathrm{H} & 2.156920610 & -2.047939864 & 0.290666549 \\ \mathrm{C} & 0.758249907 & 4.621143914 & 0.068686326 \\ \mathrm{H} & 2.831740487 & 4.017927295 & 0.227876834 \\ \mathrm{C} & -0.563705313 & 4.159055750 & -0.061661156 \\ \mathrm{H} & 0.968676985 & 5.688624515 & 0.170178520 \\ \mathrm{C} & -0.794684526 & 2.790909838 & -0.165517736 \\ \mathrm{H} & -1.406344432 & 4.853746676 & -0.084105371 \\ \mathrm{H} & -1.799592215 & 2.372184108 & -0.257980841 \\ \mathrm{Ni} & 0.00000000 & 0.000000000 & 0.00000000 \\ \mathrm{C} & -0.827411399 & 0.598328092 & 3.152074776 \\ \mathrm{O} & -0.499113179 & -1.949105488 & -0.012773436 \\ \mathrm{C} & -1.727920785 & -1.591883589 & -0.063627676 \\ \mathrm{O} & -2.000884357 & -0.340858548 & -0.123879601 \\ \mathrm{C} & -2.827709608 & -2.621033818 & -0.017483553 \\ \mathrm{H} & -2.505819670 & -3.553512585 & -0.500716807 \\ \mathrm{H} & -3.740205694 & -2.237412250 & -0.492694577 \\ \mathrm{H} & -3.059621512 & -2.851063096 & 1.035332109 \\ \mathrm{C} & -2.209503257 & 0.656477212 & 3.231298769 \\ \mathrm{C} & 0.018644803 & 0.774233852 & 4.234045867 \\ \mathrm{C} & -0.566838328 & 1.031362824 & 5.490073602 \\ \mathrm{C} & -1.961337802 & 1.100622365 & 5.613416917 \\ \mathrm{C} & -2.782648931 & 0.914306839 & 4.492566681 \\ \mathrm{H} & -2.836553314 & 0.504560775 & 2.347092021 \\ \mathrm{H} & -3.872396001 & 0.967307947 & 4.594460334 \\ \mathrm{H} & -2.411351552 & 1.299581198 & 6.590871428 \\ \mathrm{H} & 1.107418877 & 0.716884875 & 4.132496251 \\ \mathrm{H} & 0.071249047 & 1.175780298 & 6.369103775\end{array}$




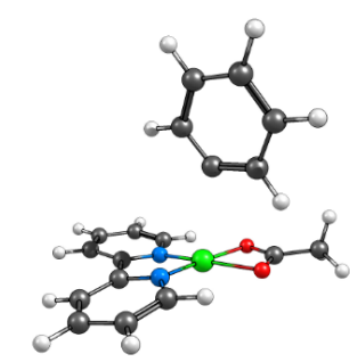

39

Aryl Carboxylate RKS 3.5 $\AA$ Ni-C distance

$\begin{array}{lrrr}6 & 4.645576000 & 0.259662000 & 0.245650000 \\ 6 & 3.836933000 & 1.393445000 & 0.192582000 \\ 6 & 4.046048000 & -1.011518000 & 0.131735000 \\ 6 & 2.668364000 & -1.097119000 & -0.030833000 \\ 7 & 1.874724000 & 0.000000000 & -0.121961000 \\ 6 & 2.453228000 & 1.252334000 & 0.003080000 \\ 6 & 1.473252000 & 2.323796000 & -0.078208000 \\ 7 & 0.175899000 & 1.863544000 & 0.067638000 \\ 6 & 1.738063000 & 3.687832000 & -0.280988000 \\ 1 & 4.269021000 & 2.389149000 & 0.321947000 \\ 1 & 5.724365000 & 0.355895000 & 0.390820000 \\ 1 & 4.644947000 & -1.924535000 & 0.161045000 \\ 1 & 2.144672000 & -2.052642000 & -0.114572000 \\ 6 & 0.682507000 & 4.595347000 & -0.332024000 \\ 1 & 2.767905000 & 4.025542000 & -0.422391000 \\ 6 & -0.636128000 & 4.114930000 & -0.199010000 \\ 1 & 0.875111000 & 5.659686000 & -0.487624000 \\ 6 & -0.844726000 & 2.752876000 & -0.017008000 \\ 1 & -1.491712000 & 4.793281000 & -0.223386000 \\ 1 & -1.842679000 & 2.322882000 & 0.091571000 \\ 28 & 0.000000000 & 0.000000000 & 0.000000000 \\ 6 & -1.099418000 & 0.670088000 & 3.239062000 \\ 8 & -0.452621000 & -1.926063000 & -0.027049000 \\ 6 & -1.693237000 & -1.600773000 & -0.046667000 \\ 8 & -1.983967000 & -0.353197000 & -0.072708000 \\ 6 & -2.766216000 & -2.654478000 & -0.003886000 \\ 1 & -2.432082000 & -3.565642000 & -0.518470000 \\ 1 & -3.696306000 & -2.280413000 & -0.451796000 \\ 1 & -2.971401000 & -2.917887000 & 1.046631000 \\ 6 & -2.480650000 & 0.575247000 & 3.247163000 \\ 6 & -0.327254000 & 0.944646000 & 4.353675000 \\ 6 & -0.997894000 & 1.139214000 & 5.578267000 \\ 6 & -2.395773000 & 1.052682000 & 5.634600000 \\ 6 & -3.137684000 & 0.772646000 & 4.478469000 \\ 1 & -3.042237000 & 0.354281000 & 2.334376000 \\ 1 & -4.230194000 & 0.705980000 & 4.528515000 \\ 1 & -2.911218000 & 1.204491000 & 6.587778000 \\ 1 & 0.764540000 & 1.010726000 & 4.302403000 \\ 1 & -0.422881000 & 1.358268000 & 6.484817000 \\ & & & \end{array}$

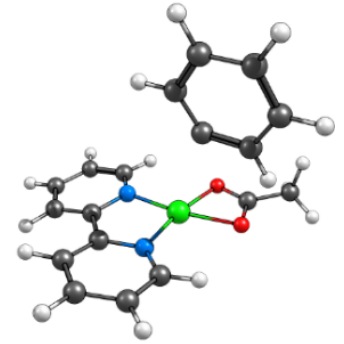

39

Aryl Carboxylate Triplet 3.6 $\AA$ Ni-C distance

$\begin{array}{lrrr}\mathrm{C} & 4.652768551 & 0.248397312 & -0.340308909 \\ \mathrm{C} & 3.844348193 & 1.384346398 & -0.291098731 \\ \mathrm{C} & 4.056010899 & -1.017748235 & -0.188400369 \\ \mathrm{C} & 2.680981863 & -1.098426156 & 0.006559091 \\ \mathrm{C} & 1.889506431 & 0.000000000 & 0.090243210 \\ \mathrm{C} & 2.466676537 & 1.245415306 & -0.068265333 \\ \mathrm{~N} & 1.490686728 & 2.328463802 & 0.015191924 \\ \mathrm{C} & 0.190431415 & 1.879190971 & -0.108025901 \\ \mathrm{H} & 4.273546151 & 3.688674627 & 0.204214770 \\ \mathrm{H} & 5.728179127 & 2.377472573 & -0.446862566 \\ \mathrm{H} & 4.653805962 & -1.931763962 & -0.510518556 \\ \mathrm{H} & 2.159462256 & -2.052004881 & -0.212841503 \\ \mathrm{C} & 0.728959238 & 4.610104282 & 0.121377657 \\ \mathrm{H} & 2.809627417 & 4.016166868 & 0.333744935 \\ \mathrm{C} & -0.593868297 & 4.143920900 & 0.138280876 \\ \mathrm{H} & 0.934752869 & 5.673240262 & 0.402467468 \\ \mathrm{C} & -0.818671440 & 2.780963536 & -0.024417271 \\ \mathrm{H} & -1.441457156 & 4.832247736 & 0.169085135 \\ \mathrm{H} & -1.822806747 & 2.359333431 & -0.111945636 \\ \mathrm{Ni} & 0.00000000 & 0.000000000 & 0.000000000 \\ \mathrm{C} & -0.958525837 & 0.555630482 & 3.394605993 \\ \mathrm{O} & -0.493288420 & -1.946969499 & -0.041814946 \\ \mathrm{C} & -1.722734606 & -1.591566987 & -0.024026769 \\ \mathrm{O} & -1.995573962 & -0.337878068 & -0.025062245 \\ \mathrm{C} & -2.821231450 & -2.621045711 & 0.031076683 \\ \mathrm{H} & -2.513744385 & -3.542616875 & -0.481278729 \\ \mathrm{H} & -3.745970422 & -2.226694062 & -0.410734826 \\ \mathrm{H} & -3.025829650 & -2.873278137 & 1.084505712 \\ \mathrm{C} & -2.339967841 & 0.540560425 & 3.315709519 \\ \mathrm{C} & -0.236297733 & 0.748748954 & 4.557901817 \\ \mathrm{C} & -0.967026090 & 0.946824568 & 5.747253016 \\ \mathrm{C} & -2.368478467 & 0.942022744 & 5.719262229 \\ \mathrm{C} & -3.055379404 & 0.740333254 & 4.513806999 \\ \mathrm{H} & -2.855978941 & 0.378154408 & 2.364567865 \\ \mathrm{H} & -4.150868868 & 0.736367142 & 4.499061578 \\ \mathrm{H} & -2.930262786 & 1.095705050 & 6.645529823 \\ \mathrm{H} & 0.858377461 & 0.749729106 & 4.570890777 \\ \mathrm{H} & -0.435222239 & 1.103616092 & 6.692116013\end{array}$




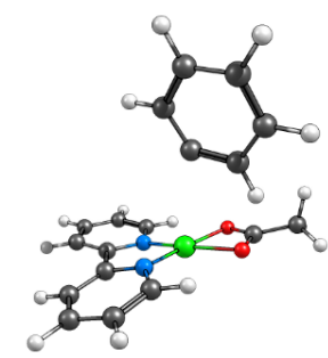

39

Aryl Carboxylate RKS 3.9 $\AA$ Ni-C distance

$\begin{array}{lrrr}6 & 4.649472000 & 0.268464000 & -0.147066000 \\ 6 & 3.835596000 & 1.400251000 & -0.153230000 \\ 6 & 4.045524000 & -1.004537000 & -0.194105000 \\ 6 & 2.659207000 & -1.094012000 & -0.246026000 \\ 7 & 1.857052000 & 0.000000000 & -0.291001000 \\ 6 & 2.442088000 & 1.254015000 & -0.231427000 \\ 6 & 1.456494000 & 2.324626000 & -0.254932000 \\ 7 & 0.177646000 & 1.869764000 & 0.020589000 \\ 6 & 1.701443000 & 3.679904000 & -0.525362000 \\ 1 & 4.273426000 & 2.398640000 & -0.072418000 \\ 1 & 5.736094000 & 0.368317000 & -0.088445000 \\ 1 & 4.646862000 & -1.916519000 & -0.199126000 \\ 1 & 2.133501000 & -2.051757000 & -0.273989000 \\ 6 & 0.644967000 & 4.588719000 & -0.511225000 \\ 1 & 2.714300000 & 4.009963000 & -0.770408000 \\ 6 & -0.656025000 & 4.115144000 & -0.247927000 \\ 1 & 0.822126000 & 5.647069000 & -0.717837000 \\ 6 & -0.846683000 & 2.759447000 & -0.006248000 \\ 1 & -1.510790000 & 4.794559000 & -0.218285000 \\ 1 & -1.832072000 & 2.335790000 & 0.199934000 \\ 28 & 0.000000000 & 0.000000000 & 0.000000000 \\ 6 & -1.182382000 & 0.981031000 & 3.631192000 \\ 8 & -0.465793000 & -1.940456000 & 0.062378000 \\ 6 & -1.691780000 & -1.600820000 & 0.202940000 \\ 8 & -1.974950000 & -0.348972000 & 0.207517000 \\ 6 & -2.765113000 & -2.639904000 & 0.391508000 \\ 1 & -2.480509000 & -3.581671000 & -0.096284000 \\ 1 & -3.726159000 & -2.280779000 & -0.000593000 \\ 1 & -2.890310000 & -2.840109000 & 1.468322000 \\ 6 & -2.521353000 & 0.749570000 & 3.378649000 \\ 6 & -0.653448000 & 1.399147000 & 4.836718000 \\ 6 & -1.562529000 & 1.605922000 & 5.894759000 \\ 6 & -2.932907000 & 1.387498000 & 5.695937000 \\ 6 & -3.414049000 & 0.962691000 & 4.449508000 \\ 1 & -2.871093000 & 0.415812000 & 2.397245000 \\ 1 & -4.486219000 & 0.793757000 & 4.301899000 \\ 1 & -3.632772000 & 1.549742000 & 6.521015000 \\ 1 & 0.418672000 & 1.566719000 & 4.979788000 \\ 1 & -1.192502000 & 1.937493000 & 6.870871000 \\ & & & \end{array}$

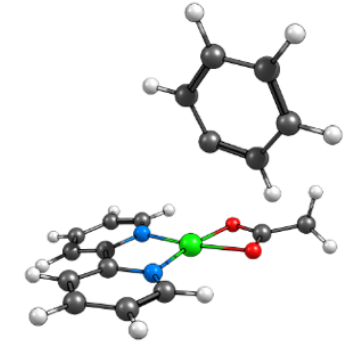

39

Aryl Carboxylate Triplet 3.9 $\AA \mathrm{Ni}-\mathrm{C}$ distance

$\begin{array}{lrrr}\mathrm{C} & 4.642398146 & 0.223090009 & -0.430936682 \\ \mathrm{C} & 3.836647745 & 1.361508339 & -0.420590304 \\ \mathrm{C} & 4.045651306 & -1.034121482 & -0.212243978 \\ \mathrm{C} & 2.674287626 & -1.102935541 & 0.008578636 \\ \mathrm{~N} & 1.885324276 & 0.000000000 & 0.056940036 \\ \mathrm{C} & 2.462197041 & 1.236710429 & -0.169357432 \\ \mathrm{C} & 1.488669968 & 2.321687944 & -0.121323889 \\ \mathrm{~N} & 0.185833521 & 1.865589278 & -0.206406549 \\ \mathrm{C} & 1.772605490 & 3.689894391 & 0.001236675 \\ \mathrm{H} & 4.264288314 & 2.345547151 & -0.629578526 \\ \mathrm{H} & 5.714997354 & 0.305791571 & -0.622910380 \\ \mathrm{H} & 4.641509833 & -1.949727903 & -0.204596740 \\ \mathrm{H} & 2.153616179 & -2.049356902 & 0.174928284 \\ \mathrm{C} & 0.726359862 & 4.611826943 & 0.023198699 \\ \mathrm{H} & 2.808433847 & 4.023808159 & 0.102651312 \\ \mathrm{C} & -0.598101136 & 4.139389829 & -0.054772135 \\ \mathrm{H} & 0.932472402 & 5.680840466 & 0.117328490 \\ \mathrm{C} & -0.823406409 & 2.770491855 & -0.149793159 \\ \mathrm{H} & -1.446069576 & 4.827900122 & -0.046222020 \\ \mathrm{H} & -1.828160829 & 2.344870187 & -0.204963158 \\ \mathrm{Ni} & 0.000000000 & 0.000000000 & 0.000000000 \\ \mathrm{C} & -1.291392700 & 0.874636817 & 3.589988220 \\ \mathrm{O} & -0.493454408 & -1.944005330 & 0.087694750 \\ \mathrm{C} & -1.721415157 & -1.585688514 & 0.112105687 \\ \mathrm{O} & -1.992777151 & -0.333114310 & 0.046365205 \\ \mathrm{C} & -2.821570882 & -2.605892205 & 0.248266375 \\ \mathrm{H} & -2.511805746 & -3.568997173 & -0.178822119 \\ \mathrm{H} & -3.741739997 & -2.250589874 & -0.234753823 \\ \mathrm{H} & -3.038031296 & -2.762939061 & 1.317753686 \\ \mathrm{C} & -2.623713155 & 0.771037899 & 3.237858390 \\ \mathrm{C} & -0.814425609 & 1.205534585 & 4.843611682 \\ \mathrm{C} & -1.773007898 & 1.459612783 & 5.845945433 \\ \mathrm{C} & -3.139615569 & 1.370743696 & 5.547249787 \\ \mathrm{C} & -3.566163855 & 1.029679019 & 4.255998107 \\ \mathrm{H} & -2.948715122 & 0.501630599 & 2.227968346 \\ \mathrm{H} & -4.636252121 & 0.961744571 & 4.031305374 \\ \mathrm{H} & -3.879474331 & 1.568494630 & 6.328607838 \\ \mathrm{H} & 0.255696618 & 1.271112725 & 5.063938275 \\ \mathrm{H} & -1.444695546 & 1.725626454 & 6.856478643\end{array}$




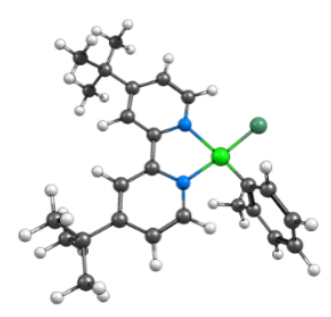

60

Aryl Chloride RKS 1.6 A Ni-C distance

$\mathrm{Ni}-0.00009519129503 \quad-0.00016310960331$

C $-2.43145763719745 \quad 1.41863184932317$

C $-2.79218288280194 \quad-0.87955592552047$

C $-3.80984294345521 \quad 1.64694475770289$

C $-4.17205284067798 \quad-0.70011716425471$

H $\quad-2.35431376070488-1.87764533291615$

C $-4.73205874349013 \quad 0.58928814213786$

H $-4.16752313526241 \quad 2.67855463662640$

H $\quad-4.79821914686812-1.59238178530806$

C $-1.42168697925816 \quad 2.48616110132030$

C $-1.70183475889974 \quad 3.85653736721692$

C $-0.66374388074989 \quad 4.80317804644603$

H $\quad-2.74145120316643 \quad 4.19012588392263$

C $0.85965547017024 \quad 2.91122269938295$

$\begin{array}{lll}\text { C } 0.64473960934390 & 4.28895882008757\end{array}$

H $1.86328949382268 \quad 2.47703457827532$

H $1.51428075555177 \quad 4.94679422047674$

C $-0.00009559771166-1.60016300372786$

C $-0.12221385815623 \quad-2.34862869860776$

C $-0.02330951103334 \quad-2.31748115982147$

C $-0.30809684522590-3.74052661513035$

C $-0.21094514897102 \quad-3.70634074695283$

C $-0.36716174035979 \quad-4.42613516656720$

H $-0.40256666639296 \quad-4.29987850124645$

H $-0.22554242021213 \quad-4.22238145923508$

Cl $2.16958162235690 \quad-0.00533808857864$

N $-1.90267773774330 \quad 0.14473981132651$

N $-0.14244973411924 \quad 2.01368476715378$

H $0.11914945114475 \quad-1.78042729741296$

H $\quad-0.51105147672642 \quad-5.51066297610340$

C $-6.24241363233700 \quad 0.86328281810150$

$\begin{array}{lll}\text { C }-0.98104397731519 & 6.30557436382766\end{array}$

C $-6.65310736158452 \quad 1.64611110658773$

H $-6.42777959660785 \quad 1.07086453742722$

$\begin{array}{lll}\mathrm{H} & -6.13272552705455 & 2.61358388823135\end{array}$

$\begin{array}{lll}\text { H } & -7.73580092532322 & 1.85235855088687\end{array}$

C $-6.57463823214545 \quad 1.70848102526103$

H $-6.05188683452903 \quad 2.67770441439613$

H $-6.29228040572331 \quad 1.17853194678159$

H $-7.65641088186042 \quad 1.91539837519315$

C $-7.06375736480908-0.43956315058676$

H $-6.83797349226754-1.03118155574553$

H $-6.89182825981827 \quad-1.07627899792377$

H $\quad-8.13831067456971 \quad-0.20010631938629$

C $-1.81257129857104 \quad 6.59738874071479$

H $-2.76676946792446 \quad 6.04791216281599$

H $-1.25829245052479 \quad 6.31898698793331$

H $\quad-2.04854575641794 \quad 7.67226980241051$

C $-1.79830773566687 \quad 6.70640281992162$

H $-1.23344728030796 \quad 6.50752878855141$

H $-2.75181103054882 \quad 6.15950145412295$

H $\quad-2.03463666097684 \quad 7.78254616573156$

C 0.29694692164003

H 0.91210110492095

H 0.92194273492345

H 0.02472404851955

C -0.02321235292252

H 0.95405575968638

H -0.79216515308397

H -0.13755440471084
6.94734411777658

7.02540584573261

8.23192113195889

$-1.66271102502664$

$-0.88021816194102$

$-2.38009037652062$
7.16583755620177

$-1.16489895884798$
$-0.00000559617899$

0.01090148403728

0.08630258146515

0.04767053979983

0.12147756022553

0.10513647032714

0.10340561047272

0.03529119683099

0.16475167365093

$-0.04585755681574$

0.10297702777062

$-0.14851345937532$

$-0.11482155355137$

$-0.07769745091348$

$-0.13279348990369$

$-0.06691450042889$

0.16408375840904

$-0.00058392471423$

$-1.21119290609299$

1.22218853849375

$-1.14825106454678$

1.26135279573674

0.07198310463159

$-2.08708388831807$

2.22753073214437

0.00036995323203

0.02915552834309

$-0.03497032567136$

2.16632049421215

0.08947739439966

0.14350559355303

$-0.21179879772830$

$-1.12863322216664$

$-2.04044926365421$

$-1.20557741884427$

$-1.11541877387570$

1.39859199209978

1.39535070233297

2.32189153445182

1.44236327985018

0.20114818309673

1.10270746584361

$-0.68138920814884$

0.22758389708582

$-1.48578652492613$

$-1.49316369714698$

$-2.39591709347576$

$-1.54690519961388$

1.04159626203189

1.96597062913101

1.10713803466336

1.01245904754398

$-0.25627521722561$

$-1.14371175771337$

0.64004590327677

$-0.30135425892844$

$-2.55757282274326$

$-2.67051430214195$

$-2.68457314132458$

$-3.38597216361538$

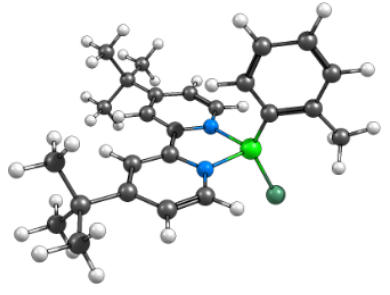

60

Aryl Chloride Triplet $1.6 \AA \mathrm{Ai}-\mathrm{C}$ distance

Ni $-0.00001814851899 \quad 0.00024948128067$

C $2.41995753548792 \quad 1.23118368271145$

C $2.72318443713820-1.06426620841106$

$\begin{array}{ll}\text { C } 3.78669336670465 & 1.38424319001933\end{array}$

C $4.08144323859412-0.96887805059321$

H $2.25537413550443 \quad-2.02087875664166$

C $4.65691523790333 \quad 0.28423741190075$

H $4.17144864340233 \quad 2.38112035823472$

H $4.68070967563701-1.87983332511796$

C $1.42511935719793 \quad 2.31126840525159$

$\begin{array}{ll}\text { C } 1.68965401396902 & 3.66083304569517\end{array}$

C $0.66472681773066 \quad 4.61835012135148$

H $2.71463552836385 \quad 3.96247971488487$

C $-0.83759823043493 \quad 2.80176560168237$

C $-0.63122565212074 \quad 4.14763052019311$

H $-1.82928539195277 \quad 2.41398423526266$

H $-1.48996997283918 \quad 4.81956246443707$

C $-0.00006088900496-0.00017432515758$

C $-0.90679192783471-0.83531661143128$

$\begin{array}{lll}\text { C } & 0.88107234251476 & 0.81100793382467\end{array}$

C -0.87440810973496

C 0.89737383101644

C 0.00876463373093

H -1.57317871109478

H 1.60171250797638

Cl -1.21044418570149

N 1.89734865248229

N 0.15555158594843

H 1.59202033119444

H $\quad 0.00138983942653$

C 6.15101468615610

C 0.97173945626243

C 6.30602433441343

H 5.86859018024529

H 5.81663309060162

H 7.37292149440754

C 6.77235539750064

H 6.29487093048741

H 6.67488014777875

H 7.84514993263563

C 6.92343517563573

H 6.87769043924177

H 6.54736284900771

H 7.98577103263421

C 1.57882677251139

H 2.51733565450395

H 0.88001233225945

H 1.80336981995968

C 1.98795032500450

H 1.58530233440629

H 2.93731783031230

H 2.21931190588506

C -0.29297780067161

H -1.04863261453991

H -0.75945320979732

H -0.02848829494889

C -1.90939070172314

H -2.61254087206837

H -1.42677019618432

H $\quad-2.49786780560347$

80581789680791

0.82569883925765

0.00731579354513

$-1.44945664361412$

1.47411765804728

$-1.11448980214495$

0.00023746476285

1.89137691241157

1.46573239857858

0.00027213230947

0.46948007169922

6.09216036216715

1.06749472148547

0.40326508007517

204964427075511

1.20509961897815

1.43667419332219

.42870541604546

1.03950694129191

1.58052024099371

$-0.86332171649925$

$-1.32874681956528$

$-1.58968791017796$

$-0.68534073307491$

6.19733496395498

5.62889504119388

5.81589540720696

7.24928305181999

6.63201628368602

6.56771715623104

6.07452682144358

7.68930918549521

6.97153347294900

6.65631779439354

6.96438540690526

8.01558745897346

$-1.75870668442517$

$-1.21656051298995$

$-2.50409449124866$

$-2.30060704680800$

0.00016081043439

$-0.83123144085451$

$-0.51181974512951$

$-1.10624220215431$

$-0.79701034713428$

$-0.26586902783566$

1.0031750859210

$-1.33331715853182$

$-0.77898330925812$

$-0.83103341905930$

1.10620004557063

$-1.09997884185452$

$-1.33381310645378$

$-0.51078499098607$

$-0.79599325010892$

$-0.26455829225082$

$-0.77769292888122$

1.60016075158076

2.34877100417521

2.36910616821349

3.75708084445163

3.76596690032336

4.47182346725578

4.30458082312950

4.29854679471734

$-1.54589838988431$

$-0.52955664390824$

$-0.52898120920058$

1.84863364019347

5.56611428530248

$-1.41196794250195$

$-1.41214862876050$

$-2.83250359176511$

$-3.59435934215682$

$-2.92403342295449$

$-3.07282950710634$

$-0.37366800631592$

0.39464701222559

0.64901844433448

$-0.58209189010635$

$-1.35848139249800$

$-0.36087509932960$

$-2.09661672119393$

$-1.58747320338789$

283338846740993

$-2.92595486630029$

$-3.59440212555015$

$-3.07410354197176$

$-0.37510950463238$

0.64805619922923

$-0.39711789001919$

$-0.58397820185112$

$-1.35737628951425$

$-2.09460527928443$

$-0.35923480079919$

$-1.58686492836864$ 


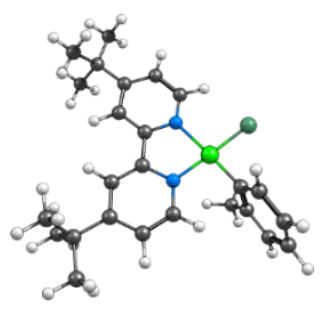

60

Aryl Chloride RKS $1.8 \AA \mathrm{Ni}-\mathrm{C}$ distance

$\mathrm{Ni}-0.00002879528857 \quad-0.00005290828175$

C $-2.39732076031478 \quad 1.41830539624174$

C $-2.78090063746139 \quad-0.87846025090131$

C $-3.77308044035989 \quad 1.66049095560419$

C $-4.15928055119254-0.68396970490465$

H $\quad-2.35259623868496 \quad-1.88058167322444$

C $-4.70672148167320 \quad 0.61117873413428$

H $-4.11927543741318 \quad 2.69621445380687$

H $\quad-4.79513731565527 \quad-1.57043133400755$

C $-1.37135685819065 \quad 2.46868182264374$

C $-1.62172029279259 \quad 3.84504172596520$

C $-0.56454975649523 \quad 4.77155620040930$

H $\quad-2.65494447684533 \quad 4.19846580379019$

C $0.92161313887808 \quad 2.84732882005648$

$\begin{array}{lll}\text { C } & 0.73293989886166 & 4.22935955743009\end{array}$

H $1.91616269847368 \quad 2.39295319142558$

H $1.61605274152883 \quad 4.86961657846533$

C $-0.00000964811992-1.80005288154110$

C $-0.06110545865039-2.54045624138021$

C $-0.06117034121954-2.49056479802610$

C $-0.21098306236977 \quad-3.93834062450885$

C $-0.20829792839914-3.88544966896525$

C $-0.29331757066434-4.61391842704291$

H $-0.25651576931811 \quad-4.50819413884838$

H $\quad-0.25003231089645 \quad-4.39763189154562$

Cl 2.16490389913508 -0.08137803512117

N $-1.87907591282569 \quad 0.13781091561163$

$\begin{array}{lll}N & -0.09951922712769 & 1.96822219672867\end{array}$

H $0.02203228724412-1.93638918476868$

H $-0.40616768753580 \quad-5.70232938152234$

C $-6.21493710219446 \quad 0.89935243067356$

C $-0.85048101731024 \quad 6.28131277302125$

C $-6.58235106861198 \quad 1.73171251041252$

H $-6.33686151885389 \quad 1.18731101005884$

$\begin{array}{lll}H & -6.05079299562125 & 2.69578348553296\end{array}$

H $\quad-7.66298942675060 \quad 1.94879296316273$

C $-6.57476385991447 \quad 1.70222752293214$

H $-6.04261664092379 \quad 2.66524449355646$

H $-6.32396062931439 \quad 1.13629199442869$

H $\quad-7.65526853850058 \quad 1.91922896302613$

C $-7.04988947929149 \quad-0.39606831784487$

H $\quad-6.85513230686808-1.02229917381229$

H $-6.85910738700478-1.00211332489085$

H $\quad-8.12248105306619-0.14659596355366$

C $-1.66770603363355 \quad 6.65427989105091$

H $-2.63285276018884 \quad 6.12565838395015$

H $-1.11339827906562 \quad 6.41055269271002$

H $-1.88139515563435 \quad 7.73552815748045$

C $-1.66728934498775 \quad 6.63574565180392$

H $-1.11260499313612 \quad 6.37870800825399$

H $-2.63226450216062 \quad 6.10627707439292$

H $-1.88118880003508 \quad 7.71672492750265$

C $0.44530513489964 \quad 7.11571120490022$

H $1.06162734976338 \quad 6.92911942907415$

H $1.06136514632763 \quad 6.91734223103630$

H $\quad 0.19558157445829 \quad 8.18822231900452$

C $0.06331837431322 \quad-1.85007190304765$

H $1.03417863650046 \quad-1.33430881552447$

H $-0.71548719806888-1.08054467895890$

H $\quad-0.01566962375261$
0.00010736647551

0.01579002381288

$-0.00294303125105$

0.02233782756931

0.00124695488195

$-0.00903770104575$

0.01472863461740

0.03411271391587

$-0.00559127275680$

0.02268378669924

0.03234581561764

0.04043904910092

0.03314418973405

0.02829000412507

0.03852919547093

0.02569438357458

0.04458823289850

$-0.00019906341853$

$-1.20741773904902$

1.22511725899322

$-1.14476842806771$

1.27061124077580

0.07946585048811

$-2.08068464280682$

2.23785056991832

0.00018634022254

0.00315253216250

0.02018076917060

2.16634372430243

0.09982178453405

0.02230360694704

0.05120697731763

$-1.23149980467300$

$-2.15695006527983$

$-1.25884990187736$

$-1.24096162533981$

1.29737263533236

1.34410688475097

2.20835438837251

1.31827115975833

0.00959942734061

0.89488840648992

$-0.89049536578464$

0.01486976275361

$-1.21078750322422$

$-1.25093429919195$

$-2.13079893862843$

$-1.21891386688970$

1.31882933393902

2.23497907670478

1.35157444874394

1.34282239650962

0.05700252743125

$-0.83690237886884$

0.94853749296922

0.06411301320013

$-2.54799550253367$

$-2.62906512263754$

2.68974203373523

$-3.38244373656241$

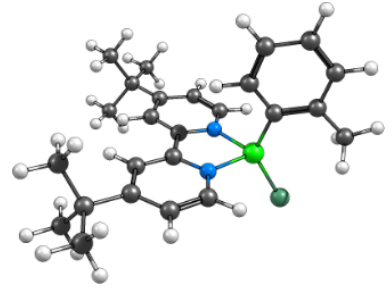

60

Aryl Chloride Triplet $1.8 \AA \mathrm{Ai}-\mathrm{C}$ distance

$\mathrm{Ni}-0.00006183850344 \quad 0.00024711447285$

C $2.44024470402626 \quad 1.22355589042359$

$2.73111674208350-1.06725417322856$

C $3.80522826954629 \quad 1.36823504012220$

C $4.09132205694173-0.98072511921332$

$\begin{array}{lll}\text { H } 2.25948909808227 & -2.01913729514657\end{array}$

$4.67155071593231-0.26382671534331$

H $4.19403299123585 \quad 2.36049218707771$

H $4.68742999671712 \quad-1.89335498080987$

C $1.45056142817960 \quad 2.31271774305159$

$1.72487818203026 \quad 3.65766524009005$

C $0.70827169565455 \quad 4.62552597535164$

H $2.74968772338891 \quad 3.94990252903756$

C $-0.80190739572664 \quad 2.82101075957172$

C $-0.58592115942905 \quad 4.16677626343574$

H $-1.79443289654528 \quad 2.44243112992562$

H $-1.43744898788985 \quad 4.84729094122924$

$\begin{array}{ll}-0.00014909714491 & -0.00005227196299\end{array}$

C $-0.89659980639900-0.81475297161792$

C 0.89694504740106

$-0.85172438572350$

C 0.93306115482571

C 0.04874224784062

H -1.54661970554407

H 1.64805008168858

Cl -1.24546001245444

N 1.90995550153085

N 0.18223971222379

H 1.60289430321331

H 0.05745705097425

C 6.16560132737820

C 1.02512852695281

C 6.31988369259030

H 5.87917964212969

H 5.83374763899965

H 7.38681349256427

C 6.79187468316309

H 6.31831414682301

H 6.69461047591251

H 7.86479355173248

C 6.93296848884858

H 6.88748397075661

H 6.55284428342061

H 7.99549558165485

C 1.61328651860416

H 2.54463868485065

H 0.89999742567418

H 1.84513051979310

C 2.06112950990159

H 1.67241529118712

H 3.00477989607566

H 2.29962722186481

C -0.23008389756955

H -0.99915949700804

H -0.68232817105357

H 0.04129838307982

C -1.90380424051790

H -2.59935335355294

H -1.42067635403350

H -2.50143465337890
0.00001133050884

$-0.77226673773056$

$-0.43137553311302$

$-1.05399847346495$

$-0.71443326551062$

$-0.17459192278445$

$-1.03548247047599$

$-1.29365741824276$

$-0.68040212045696$

$-0.77213352901019$

$-1.05380589597672$

$-1.03495876113198$

$-1.29374435637438$

$-0.43067369978277$

$-0.71357691668393$

$-0.17372693622958$

$-0.67926272421595$

1.80001130286397

2.55438334639711

2.52539741699421

$-0.77411889272742 \quad 3.96202535223915$

$0.84756623855019 \quad 3.92513270088251$

$0.04400576703272 \quad 4.65156404827941$

$-1.40564741437365 \quad 4.52844441331419$

$1.49712146926642 \quad 4.44209532754983$

$-1.13177326274131 \quad-1.46176173818456$ $0.00022630377906 \quad-0.46086236743909$

1.90163158026701

$1.45645802022791 \quad 1.98206344186459$

$0.05178391043647 \quad 5.74615847090916$

$0.43884367019327-1.35211204926111$

$6.09606223890002-1.35162916794198$

$1.01577498454604 \quad-2.78144889591104$

$0.34201448677489 \quad-3.53298471156927$

$1.99820087935146 \quad-2.88646680076304$

$1.14628042840080-3.02531703100417$

$1.41877136531359-0.32876959823823$

$2.41229747340861-0.36346013990230$

$1.03691761719856 \quad 0.69971771556380$

$1.55541565546284-0.54101944145874$

$-0.89596731974394 \quad-1.28056482286522$

$-1.34656256936111-0.27617571303283$

$\begin{array}{ll}-1.63159613526717 & -2.00736987914345\end{array}$

$-0.72535246481566 \quad-1.51404814658409$

$6.19459686204678-2.78129659012769$

$5.61671999967429 \quad-2.88693521182939$

$5.82046819927039 \quad-3.53244858915214$

$7.24416711931180 \quad-3.02519959701200$

$6.62563194909790 \quad-0.32891588566016$ $6.56523174983047 \quad 0.69982532475228$ $6.05921167317627 \quad-0.36431507150456$ $7.68058616600918-0.54121105595587$ $6.98760318051929-1.27923961564910$ $6.67965508583302-2.00558654188438$ $6.98538840977913-0.27456127560633$ $8.02893378242890-1.51284372387608$

$-1.73019249815296 \quad 1.88906086606668$ $-1.17838260668677 \quad 1.23721234779066$ $\begin{array}{ll}2.47542102589422 & 1.23759662483967\end{array}$ $\begin{array}{ll}-2.27307739626479 & 2.63803678533353\end{array}$ 


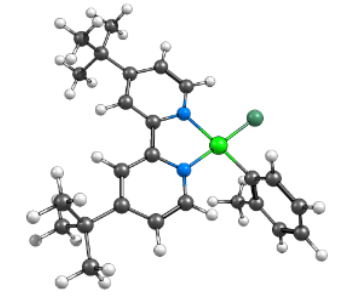

60

Aryl Chloride RKS Fully Optimized

Ni $1.25914263866544 \quad-1.29757372487669 \quad-0.14112525437629$

C $-0.53174202130060 \quad 0.82236263138977 \quad-0.05276765965357$

$\begin{array}{llll}\text { C } & 1.66882229381741 & 1.58482149937974 & -0.09475688098736\end{array}$

$\begin{array}{llll}\text { C } & -1.00041811644866 & 2.13755601376627 & -0.01594938200899\end{array}$

C $1.24638321690200 \quad 2.91079811341753 \quad-0.05677506044416$

H $2.72774103352657 \quad 1.32971435780501 \quad-0.13107327951978$

$\begin{array}{llll}\text { C } & -0.12169966702071 & 3.23352499929650 & -0.01636271773084\end{array}$

H $\quad-2.07925406113239 \quad 2.30512352686027 \quad 0.01374107432126$

H $2.01391709480731 \quad 3.68608976935289 \quad-0.06033122930693$

C $-1.39263143480114 \quad-0.36642486475297 \quad-0.05295364225741$

C $-2.79074638678948 \quad-0.35569668576642 \quad-0.01574421366813$

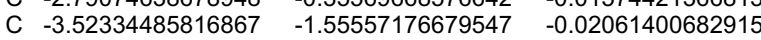

H $\quad-3.31453146242078 \quad 0.60196657867422 \quad 0.01773258177511$

C $-1.37443268109171 \quad-2.69117663415023 \quad-0.10054220131032$

$\begin{array}{llll}\text { C } & -2.76780579930842 & -2.74084937248017 & -0.06487415953652\end{array}$

H $\quad-0.75731215719097 \quad-3.59314116075665 \quad-0.13480541898368$

H $-3.24785264820125 \quad-3.72032346798859$

C $3.12167799417186-0.98525023074852$

C $3.88713966266663-0.84006451036810$

C $3.73442935142503-0.78892449449916$

C $5.24206114585184-0.47418450938750$

C $5.08769209787184-0.42730317983252$

C $5.84336057278074-0.26077583456522$

H $5.83796598690090 \quad-0.36284189962233$

H $5.54677431674462-0.28304535751910$

Cl $1.72076309007112 \quad-3.41224871316937$

N $0.81734183325104 \quad 0.52585068203240$

N $-0.68231175198931 \quad-1.53458309165407$

H $3.15763893991219-0.93357642425723$

H $6.89988598270750 \quad 0.01894601327378$

C $-0.65734502626263 \quad 4.67204552570972$

C $-5.05921626916135-1.53232244816780$

C $-1.49469553694754 \quad 4.86914370827726$

H $-0.88484449529059 \quad 4.69914097904711$

H $-2.35520170679103 \quad 4.18334406818115$

H $-1.88824327947235 \quad 5.89779649575126$

C $-1.55320168785508 \quad 4.91876094207600$

H $-2.41524944149532 \quad 4.23419170433869$

H $-0.98577866310069 \quad 4.78494194026435$

H $\quad-1.94763424698216 \quad 5.94795307964326$

C $0.48051932323975 \quad 5.71159325100046$

H 1.09909773993250

H 1.14096453079650

H 0.05588365268223

C -5.52387743589316

H $\quad-5.16680567530302$

H $\quad-5.15835532455597$

H -6.62484696915958

C -5.59021531154789

H -5.27289080024962

H -5.23486465871328

H -6.69203617921610

C -5.65991034716769

H -5.34110698622560

H -5.38917654798001

H $\quad-6.75893994070766$

C 3.27652035100237

H 2.91963209269041

H 2.40209888798489

H 4.00137774553978
5.64243184315293

5.60657776911028

6.72720953839913

$-0.8130392285780$

0.22757305102885

$-1.33312100462607$

$-0.78800634997499$

$-0.76660623867138$

$-1.25302899077683$

0.27528739372898

$-0.74088870301986$

$-2.95161252088294$

$-3.54215257144896$

$-3.50913605271111$

$-2.88927610549583$

$-1.10068026789563$

$-2.14124019976414$

$-0.45384149149462$

$-0.93107637825930$
$-0.07211723022273$

$-0.21289635542658$

0.96707410642455

$-1.46139520337966$

0.85633735540310

$-1.55596047625282$

1.77020551289907

$-2.53982302287140$

$-0.19215085325278$

$-0.09374530448884$

$-0.09506518765389$

$-2.38121911477150$

$-0.44922887060878$

0.02450604903262

0.02036859476526

1.31285159728213

2.21411461062482

1.35630102700777

1.35739925369609

$-1.21503149159144$

1.24560349843737

$-2.14948079092735$

$-1.20086016146429$

0.01864066976738

$-0.89043370459579$

0.89422305831025

0.04896934929135

1.31123275116574

1.35733578111832

2.21066792518388

1.35671367346684

$-1.21713818715370$

$-1.24373330169090$

$-1.20389692229145$

0.01016972473181

0.88387603002167

$-0.90064927672812$

0.04085694122440

2.32611105203635

2.39589697590361

51466279390370

3.13807883881008
$-0.39062653672574$

$-2.15296073101891$

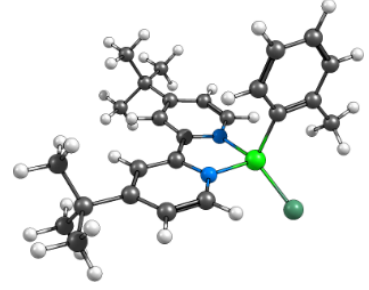

60

Aryl Chloride Triplet Fully Optimized

$\mathrm{Ni}-0.23540777135903 \quad 0.31018446026536$

C $2.29083220390681 \quad 1.34518716335624$

$2.41894178782609-0.95941701734680$

$\begin{array}{ll}3.67862265222037 & 1.40681453530990\end{array}$

C $3.79551904156790-0.95610423959661$

H $1.88527657974779-1.88410687668948$

C $4.47396337289971 \quad 0.25251156758757$

$\begin{array}{lll}H & 4.14289137231177 & 2.37915759083879\end{array}$

H $4.32971735121659-1.90484279452097$

C $1.38209920467270 \quad 2.49849260938635$

C $1.74031281367590 \quad 3.80991529531082$

C $0.79120954430733 \quad 4.84434481210330$

H $2.77527271715399 \quad 4.02080757817278$

C $-0.83262578609093 \quad 3.16770319808593$

C $-0.52576622945333 \quad 4.48674680618784$

H $-1.84507684647038 \quad 2.85345034800304$

H $-1.32674288603741 \quad 5.22628783262283$

$\begin{array}{ll}-0.00426178361110 & 0.03714090469346\end{array}$

C $-0.34056591998319-1.20678524282082$

C $0.50687966242832 \quad 1.05698112348022$

C $-0.16578561154844 \quad-1.37757882343230$

$\begin{array}{lll}\text { C } 0.66858535963986 & 0.87839459568668\end{array}$

C $0.32968658980627-0.34762781558011$

H -0.42894601153568

H 1.05973915161548

Cl -2.29838784081982

N 1.65736749083207

N 0.09150037144065

H 0.78326605897266

H 0.45344245570863

C 5.99500307552973

C 1.19915686901311

C 6.29212938093399

H 5.86971382619168

H 5.87572618815676

H 7.38036832994346

C 6.60372146201723

H 6.19728418621283

H 6.40471099864452

H 7.69616942960706

C 6.66971524315749

H 6.52602650002621

H 6.29483174932957

H 7.75464638879000

C 1.74869187991611

H 2.63387251644871

H 0.98820308806235

H 2.04505921823988

C 2.30005538547534

H 1.94000784538702

H 3.20140723071409

H 2.60337413407765

C 0.01140567702712

H $\quad-0.79774873045797$

H -0.41110615459666

H 0.34755060988619

C -0.90722348730093

H -1.92109958077136

H -0.30747087102689

H -0.95539868367601

.34097653800853

1.69510656527290

$-0.05305797102294$

0.15399977748992

2.18567235375279

2.02334074500650

$-0.50655411562674$

0.33854120901088

6.27752324448861

0.93777174673205

0.31055726178903

1.95086322895679

1.00736067340604

1.25167435246038

2.27421413254278

0.85357102548860

1.32315793809600

$-1.04438094113250$

$-1.51214043038746$

$-1.73829436734654$

$-0.93844340051619$

6.28533669297192

5.63979377123149

5.93691558667562

7.30705903133254

6.76887038900993

6.77137308892836

6.13773556583065

7.79682795560992

7.25697007617969

6.98076211464726

7.31830032807505

8.26869912956233

$-2.34152433496148$

$-2.10460881984998$

$-2.53984410050117$

$-3.27974277629868$

0.13459255983897

$-0.59101766611350$

$-0.25254800890352$

$-0.77180375522411$

$-0.44849690163390$

$-0.01892292753061$

. 71426446802925

$-0.95208714706508$

$-0.38444273600487$

$-0.63322982142253$

0.97339327091469

$-0.96910637049882$

$-1.25200158271982$

$-0.28797819363013$

$-0.60996933761666$

$-0.02328745335014$

$-0.58093048412576$

2.04627432835091

2.63175418766855

2.86358650884769

01850902790557

4.24660042341246

4.82664292113218

4.47196327789024

4.86323368198969

$-0.53980969461748$

$-0.32945459299786$

$-0.29926766590483$

2.42577268559753

5.90253052610902

$-0.91730410703323$

$-1.34738072779932$

$-2.31444038628769$

$-3.11532007825794$

2.42745052436924

$-2.47519715448153$

0.17613215913840

0.13429841238705

1.18349088805518

0.04739670325123

$-0.82787951857095$

0.15922973123088

$-1.59735783938977$

$-0.98396827282538$

2.79575727092863

$-2.90649865716876$

$-3.51218558099945$

$-3.08422344743124$

$-0.37466135160884$

0.66625635952401

$-0.41603169383638$

$-0.63192425499973$

$-1.27227142158854$

$-1.96704654621960$

$-0.25659273320957$

$-1.54808054264051$

1.80382343379753

1.44365189768880

0.89816971590410

2.37860755822987 


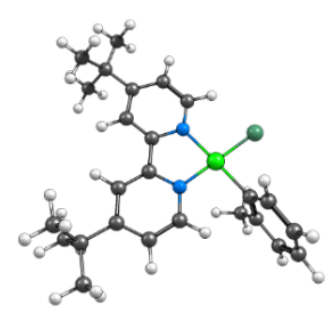

60

Aryl Chloride RKS 2.0 A Ni-C distance

Ni $0.00000469694548 \quad-0.00009997227649$

C $-2.37760056884335 \quad 1.41727920835087$

C $-2.77045973186884-0.87895611815654$

C $-3.75164586504257 \quad 1.66630688281888$

C $-4.14802011858258-0.67684586400380$

H $\quad-2.34435367830657 \quad-1.88198883543815$

C $-4.68957198592140 \quad 0.62078025041478$

H $\quad-4.09301128117879 \quad 2.70361935192857$

H $-4.78822158910819-1.55986265289561$

C $-1.34240882756277 \quad 2.45699801839454$

C $-1.57503680927772 \quad 3.83543448094200$

C $-0.50670812082467 \quad 4.74901521768178$

H $\quad-2.60435587144616 \quad 4.20038056378347$

C 0.95781436255680

C 0.78412875630896

H 1.94666467673261

H 1.67488947235841

C -0.00001632789204

C -0.02012885614027

C -0.07031331302220

C -0.13857273033766

C -0.18457049710683

C -0.22616645059369

H -0.15325907518946

H -0.23429303812089

Cl 2.15946269551495

N -1.86504529162909

N -0.07471894124208

H -0.02094998035739

H -0.31272351160995

C -6.19656040501340

C -0.77425963948047

C -6.54755462667185

H -6.29508242788228

H -6.01141853024581

H -7.62705868839458

C -6.56595654801316

H -6.03002292830702

H -6.32707494522342

H -7.64562928399987

C -7.03709791074747

H -6.85394849326599

H -6.84010968602928

H -8.10856956834404

C -1.58149672993214

H -2.55276758065917

H -1.02622931192040

H -1.78215482823594

C -1.59209900462414

H -1.04449582930668

H -2.56356618128382

H -1.79300543293403

C 0.53162786905479

H 1.14938439447017

H 1.14142235291085

H $\quad 0.29509696673774$

C 0.11060594781991

H 1.07117311143668

H -0.68227971354287 2.80737560068764

4.19071891629386

2.34078361264146

4.82004990089809

$-2.00009972232385$

$-2.72290456895650$

$-2.67921623913860$

$-4.12519338483461$

$-4.07863670098201$

$-4.80303165833842$

$-4.69507203686280$

$-4.59641807121572$

$-0.13562291729621$

0.13398157600531

94068174232352

$-2.12067177556297$

$-5.89403779965295$

0.91566661570710

6.26166625030509

1.76519890358627

1.23120312314034

2.72706117102001

1.98738037171525

1.70429102516051

66420073339927

1.12594033750005

1.92603917370448

$-0.37564443115816$

$-1.01387905006622$

$-0.97115538265904$

$-0.12136389431913$

66566995496951

6.14950637082931

6.43057173776585

7.74941693141233

6.60520049152552

6.32628923262757

6.08707851673134

7.68817147777077

7.07979828861942

6.90057763354312

6.85891434960803

8.15505263010846

$-2.01626206115483$

$-1.47879321163403$

$-1.26161650169875$

$-2.72161115428897$
00087217079841

0.01773232853815

$-0.03519455150432$

0.01378225958158

$-0.04210753505676$

$-0.04947991261306$

$-0.01681548112567$

0.03466341987198

$-0.06704145623046$

0.04701564162629

0.07832831773572

0.10567464750656

0.08155604705070

0.06748327481835

0.09958917615859

0.06128315280620

0.11936454055136

$-0.00012979488640$

$-1.21111623172477$

1.22354825203490

$-1.15579829520696$

1.26295875178739

0.06697044767034

$-2.09262289348246$

2.22678944586630

0.00192944783325

$-0.00589892432436$

0.04114911303554

2.16424666079796

0.08283715528318

$-0.02089649842996$

0.14047349899052

$-1.26789652145739$

$-2.19752338874683$

$-127785985127522$

$-1.28560182594258$

1.26031634598015

1.32449482961533

2.16669623410137

1.27286954984035

$-0.05822955565903$

0.82095640074659

$-0.96400707253740$

$-0.06041944514725$

$-1.11841274042813$

$-1.17132805376676$

$-2.04008983302800$

$-1.10935213402922$

1.41045126588311

2.32445782221568

1.43050585035732

1.45148548210153

0.16544411062333

$-0.72898438423247$

1.05599503288331

0.18953930797021

$-2.54134945277335$

$-2.59911321840509$

2.68322763964503

$-3.38588191600260$

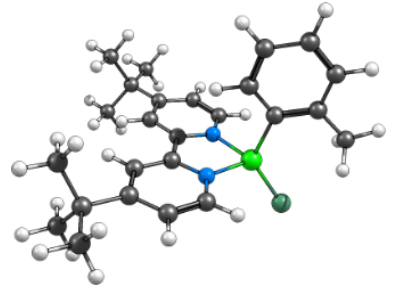

60

Aryl Chloride Triplet $2.0 \AA \mathrm{Ai}-\mathrm{C}$ distance

Ni $-0.00034345668129 \quad 0.00061355447811$

C $2.46889925532005 \quad 1.22622156571255$

$2.73694470220438-1.07171742048560$

$\begin{array}{ll}3.84185611060566 & 1.36845734018708\end{array}$

C $4.10628936918074-0.98709089276282$

H $2.25309056655512-2.02557566408600$

$4.70211696811821 \quad 0.25906108680401$

$\begin{array}{lll}\text { H } & 4.24276325416537 & 2.36277805232745\end{array}$

H $4.69701519833842 \quad-1.90299554129494$

C $1.48574345882195 \quad 2.32251586272661$

$1.77601214753516 \quad 3.67198839555794$

C $0.76649371205768 \quad 4.64753896369734$

H $2.80788403782996 \quad 3.96263545637884$

C $-0.76943925545692 \quad 2.83821561182004$

C $-0.53684126958926 \quad 4.19034560346113$

H $-1.77010127931126 \quad 2.46065437584866$

H $-1.38326775018183 \quad 4.87689716445868$

$\begin{array}{ll}-0.00036773057894 & 0.00019495810425\end{array}$

C $-0.89320151114610-0.80211789094444$

$\begin{array}{lll}\text { C } & 0.90988101996382 & 0.81792585575549\end{array}$

$-0.83627355207572$

C 0.95943715992983

C 0.07724861643232

H -1.52831053495726

H 1.68233412227004

$\mathrm{Cl}-1.30486227471549$

N 1.92147850125752

N 0.20783495526861

H 1.61169432667180

H 0.09742227918919

C 6.20597516544790

C 1.10096314605058

C 6.40708467666430

H 5.98748692165207

H 5.92845177855140

H 7.48161635240493

C 6.80462295214590

H 6.33746789727781

H 6.67319546494444

H 7.88432270998841

C 6.96455105745324

H 6.88596778877119

H 6.60370939103466

H 8.03456023338107

C 1.71798590268913

H 2.64641622428714

H 1.01652331318851

H 1.96334376067193

C 2.12070458814878

H 1.71158098026539

H 3.06072196835149

H 2.37105750448878

C -0.14847439018856

H -0.90557771360754

H -0.62026261814014

H 0.13547601411606

C -1.90516122525292

H -2.59495351022885

H -1.42275805800774

H -2.50788307457110

0.86234528740107

0.06970073411054

$-1.37292788670870$

1.51180474537954

$-1.16970514647242$ 0.00037703947582

1.91124928766682

1.44854849729526

0.08774696271767

0.43153004467523

6.12384788627173

1.03065694865497

0.37101862353937

01685871570700

1.16024796411433

1.39170138022171

2.38829551529126

0.99419066594537

1.52576531028102

$-0.90815997182948$

$-1.37446200659692$

$-1.63015791368050$

$-0.73929472584114$

6.25874105782502

5.67593491365420

5.91317170545532

7.31286136153684

6.61482806014828

6.52732668602134

6.04231853739409

7.67361012352097

7.02330887196733

6.74291668876392

6.99585444497601

8.06865648685857

$-1.71163646889006$

$-1.15280144413252$

$-2.45737223132255$

$-2.25289203181426$

0.00023063429619

$-0.63755907947922$

$-0.33176538577725$

$-0.87621244344759$

0.56810402871228

$-0.10694158705557$

.

1.08539927449304

$-0.52844575365048$

$-0.63722751856977$

0.87568236880922

$-0.84948566793619$

$-1.08533242751625$

- 33059106156386

$-0.56671690018516$

$-0.10558147949599$

$-0.52670279927419$

2.00023059148485

2.75417914189026

2.69057202563583 


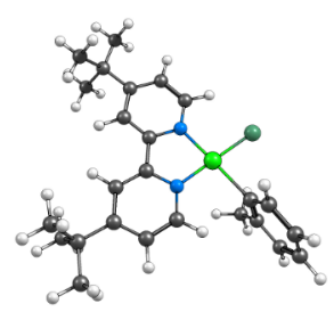

60

Aryl Chloride RKS $2.2 \AA \mathrm{Ni}-\mathrm{C}$ distance

$\mathrm{Ni}-0.00020430810608 \quad-0.00010885143142$

C $-2.36772706803534 \quad 1.41263124766689$

C $-2.76111593049140 \quad-0.88493750859964$

C $-3.74124272797367 \quad 1.66280831310891$

C $-4.13863867340359-0.68097197142210$

H $\quad-2.33257052172348 \quad-1.88700267695247$

C $-4.67909380605197 \quad 0.61712553728032$

H $-4.08234245904608 \quad 2.70018386940789$

H $\quad-4.77981696338199 \quad-1.56300979200671$

C $-1.32807316595997 \quad 2.44737451243816$

C $-1.55144717020819 \quad 3.82657274076674$

C $-0.47741191879499 \quad 4.73348817946649$

H $\quad-2.57879530154462 \quad 4.19724922105167$

C 0.97668893792058

C 0.81009950567699

H 1.96245091636072

H 1.70456379692338

C -0.00019858601387

C 0.02368602566605

C -0.07948882212016

C -0.06462879613247

C -0.16285434223405

C -0.16099481719940

H -0.04708682973703

H $\quad-0.22145573899502$

Cl 2.15489840318190

N -1.85696284510281

$\mathrm{N}-0.06194192691582$

H -0.06386375823417

H -0.22258842604427

C -6.18601865664888

C -0.73575978129019

C -6.53189614657673

H -6.27614682123619

H -5.99530009855900

H $\quad-7.61122021420637$

C -6.55995039064366

H -6.02388274652326

H -6.32479846089185

H -7.63955846054900

C -7.02706421778727

H -6.84748150695536

H -6.82715802739621

H -8.09837989116456

C -1.53911724301471

H -2.51344974377215

H -0.98426159727594

H -1.73324547148998

C -1.55290485019419

H -1.00803215181008

H -2.52754163828376

H -1.74730533673210

C 0.57509695633606

H 1.19269343268100

H 1.18257136324975

H 0.34515512614717

C 0.16546178495899

H 1.11490524036746

H -0.64074252932657

2.78346334705273

4.16724491444783

2.31031921030274

4.79113521140960

2.20010881363469

$-2.90062864832187$

$-2.87638944878257$

4. 30644058427257

$-4.27952627922173$

$-4.99323872761875$

$-4.87066189833880$

$-4.80728788281421$

$-0.17341036069633$

0.12882801739125

92357028186996

$-2.32033393583180$

$-6.08607815453633$

0.91273463240833

6.24738448757765

1.76963567756176

1.24089961606071

73128590720645

1.99243468682339

1.69409738462972

.65338917420857

1.11039391985988

1.91627611502677

$-0.37788180466004$

$-1.02138739107574$

$-0.96815942690543$

$-0.12300004685966$

.66591051729423

6.15607013888060

6.43441941157960

7.75075522380032

6.58647097340481

6.29740349129348

6.07415178862869

7.67030704550074

7.05725927656733

6.88107599456010

6.82585762167482

8.13373431043382

$-2.17294970471088$

$-1.61401691696514$

$-1.43314949556858$

$-2.86550222641551$
0.00011653007981

0.01912876933990

$-0.05032336420421$

0.01126848322988

$-0.06111314447446$

$-0.06881045425665$

$-0.02949708119960$

0.03686830502007

$-0.0940347802405$

0.05829530537055

0.09980129930583

0.13502834403719

0.10504845122070

0.08360476919629

0.12588822046629

0.07497042275438

0.15120680302216

$-0.00027780484087$

$-1.21841124447639$

1.21903565338916

$-1.17578814110347$

1.24473466932703

0.04155925442569

$-2.11600690510610$

2.20267101445465

0.00014531984164

$-0.01057747742215$

0.04974257157127

2.16200077995777

0.04787778485372

$-0.03754544597209$

0.18101755651746

$-1.28094249274186$

$-2.21267671976453$

$-1.28331649151164$

$-1.30145108152572$

1.24680051006596

1.31860029789622

2.15072283287042

1.25651822004426

$-0.08553463799605$

0.79054545510732

$-0.99409544207151$

$-0.09011655416417$

$-1.07563013542105$

$-1.13349856791976$

$-1.99846650956637$

$-1.05857298254270$

1.45265014220476

2.36512877063263

1.46780196838381

1.50156543165430

0.21354878106577

$-0.68159699423144$

1.10301749933517

0.24559141132828

$-2.53509492134431$

$-2.56391543448657$

2.67875740081148

$-3.39135720457467$

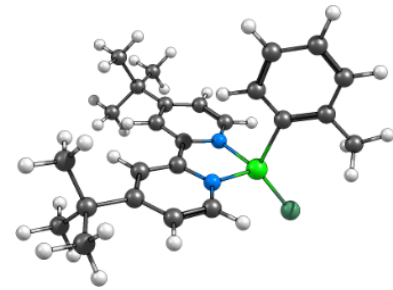

60

Aryl Chloride Triplet $2.2 \AA \mathrm{Ai}-\mathrm{C}$ distance

$\mathrm{Ni}-0.00168795236911 \quad 0.00147877474143$

C $2.49494127830570 \quad 1.23123909595727$

$2.73803566586097-1.07694924705991$

$\begin{array}{ll}3.87750929250232 & 1.37258650537521\end{array}$

C $4.11719840346384-0.99280204897540$

H $2.24028564021506 \quad-2.03456181353572$

$4.73080333325739 \quad 0.25776622866245$

$\begin{array}{lll}H & 4.29202425047218 & 2.37068504945669\end{array}$

H $4.70157534774466-1.91277387546175$

C $1.51781189372609 \quad 2.33324064466647$

$1.82359350085115 \quad 3.68903675497000$

C $0.81898102169825 \quad 4.66969570665970$

$\begin{array}{lll}\text { H } & 2.86409559017251 & 3.98100396371527\end{array}$

C $-0.74451848771279 \quad 2.85078870616602$

H -1.75475396583176

H -1.33930766309124

$-0.00112220095476$

C -0.89090629288884

C 0.91779654937010

$-0.82311423491192$

C 0.97716431498702

C 0.09836028572337

H -1.51114442692586

H 1.70453577514805

$\mathrm{Cl}-1.37084111733222$

N 1.92898552066891

N 0.22825834189649

H 1.61440740821495

H 0.12725642968584

C 6.24590987024988

C 1.17075745381967

C 6.51351901775196

H 6.12732401764716

H 6.04379213052935

H 7.59689759036659

C 6.80044186147002

H 6.34000125965751

H 6.62096646254770

H 7.88765662991181

C 6.99432956553235

H 6.86890148314642

H 6.66422172487590

H 8.07291165488142

C 1.83884248421010

H 2.76689940027003

H 1.16355202498415

H 2.09783677592056

C 215452357178347

H 1.70888568775140

H 3.09234700770605

H 2.41617475054597

C -0.07569304804324

H -0.80719881352772

H -0.58265945525858

H 0.22066580090798

C -1.90457898485349

H -2.59139966395365

H -1.42093763731604

H -2.50849022510289

4.21006792363452

2.47121562839374

4.90030880052190

0.00213657754503

$-0.79447380455247$

0.82475202005497

$-0.73329786863918$

0.87826875583359

0.09189183594528

$-1.34916974604554$

152948937565452

$-1.21391037454054$

0.00051107494337

91854005559606

1.44806490966966

0.11812044672931

0.42961607284328

6.15347449814441

1.07133634457926

0.43826751760637

2.06274619625509

1.20180458193798

1.35287381193022

2.35287855814674

0.92481810985568

1.48570814114367

$-0.91616460152955$

$-1.41253308310407$

$-1.61246331606148$

$-0.74766489642556$

34294219064965

5.75804496185942

6.03549619807940

7.40299183505412

6.59303295190917

6.46535948668777

6.01647872574201

7.65667390537057

7.05758058966448

6.81359659205673

6.99218922943577

8.10827264932158

$-1.70292706969298$

$-1.14129733262553$

244825640411581

$-2.24289075280037$

00013145350349

$-0.46611288519532$

$-0.22304323376706$

0.63939500192906

$-0.39280778351405$

$-0.05040240285972$

.61007787957303

.79805477818693

$-0.35317013015462$

$-0.46568368052562$

$-0.63842627263545$

$-0.60799148290531$

$-0.79763587526572$ 


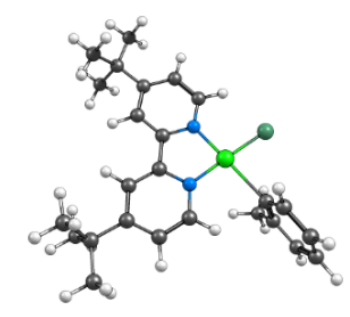

60

Aryl Chloride RKS 2.4 A Ni-C distance

$\mathrm{Ni}-0.00009344637744 \quad-0.00001549369818$

C $-2.36523535235569 \quad 1.40251409358776$

C $-2.75178095078939-0.89756081985090$

C $-3.73916839247594 \quad 1.64920447172649$

C $-4.12986701681762-0.69641081721943$

H $\quad-2.31739680060980-1.89717427450826$

C $-4.67341759840319 \quad 0.60039556548713$

H $-4.08365012633806 \quad 2.68538718583475$

H $\quad-4.76909042454337-1.57951081982065$

C $-1.32530070916455 \quad 2.43676691508216$

C $-1.54654226578502 \quad 3.81560758102072$

C $-0.47143068990345 \quad 4.72112938930102$

H $\quad-2.57366773608930 \quad 4.18704720289218$

C $0.98176602996038 \quad 2.77020827676579$

C $0.81559794741930 \quad 4.15357287524885$

H $1.96682417081038 \quad 2.29560760545681$

H $1.71060574387759 \quad 4.77643308598882$

C $-0.00001335664635 \quad-2.40001543592967$

C $0.08002914720689-3.07478813888479$

C $-0.10206270469905-3.08034544922630$

C $0.02089859740762 \quad-4.48332545331324$

C $-0.15557320181468 \quad-4.48635625172908$

C $-0.09823678060109-5.18421116133575$

H $0.08016416121932 \quad-5.03731049088852$

H $\quad-0.23356685563548 \quad-5.02716525633020$

Cl 2.15188210200149 -0.19706988246690

$\begin{array}{lll}\mathrm{N} & -1.85236149771034 & 0.12009336406372\end{array}$

N $-0.05846609206666 \quad 1.91206981115892$

H $\quad-0.13008160508690 \quad-2.53202364230502$

H $\quad-0.13574417194687 \quad-6.27814257131324$

C $-6.18121396826898 \quad 0.89185875352877$

C $-0.72823987605919 \quad 6.23483013056674$

$\begin{array}{lll}\text { C } & -6.52398663111751 & 1.75521548517368\end{array}$

H $-6.26262587665493 \quad 1.23278717514391$

H $\quad-5.99017008430527 \quad 2.71839211871372$

$\begin{array}{lll}\mathrm{H} & -7.60383481746910 & 1.97507346249947\end{array}$

C $-6.56295635583676 \quad 1.66447245375376$

$\mathrm{H}-6.02994321020682 \quad 2.62481958163152$

H $-6.33016254042300 \quad 1.07601909610390$

H $\quad-7.64320939665312 \quad 1.88358096961114$

C $-7.01849306988093 \quad-0.40071726874575$

H $-6.84108710773840-1.04898612064609$

H $-6.81303690686654-0.98500144858320$

H $\quad-8.09045549561761 \quad-0.14867748248548$

C $-1.52943162452614 \quad 6.66479423469053$

H $-2.50421575325364 \quad 6.15647635530163$

H $-0.97354481844433 \quad 6.44050311812419$

H $-1.72250294103865 \quad 7.74965216035646$

C $-1.54676314047577 \quad 6.56423065562448$

H $-1.00345894359360 \quad 6.26697261511327$

H $-2.52198306290810 \quad 6.05288031638795$

H $-1.74008869346372 \quad 7.64782830934104$

C $0.58344308734705 \quad 7.04297633314028$

H $1.20207158688560 \quad 6.87369458484992$

H $1.18945532730014 \quad 6.80345630035785$

H $\quad 0.35461068120466 \quad 8.11938885873300$

C $0.24693253513691 \quad-2.32271807746427$

H $1.18475693172488-1.74410274833806$

H $\quad-0.56983977823738 \quad-1.59668586546177$

H 0.26674457339750
$-2.99977276381763$
$-0.00062395488460$

0.01921321855469

$-0.06621698969251$

0.00693148646056

$-0.08161921108961$

$-0.08835434959737$

0.04437644852338

0.03704117720456

$-0.12258782505535$

0.06889452853653

0.12120476613187

0.16524312934315

0.12840439069675

0.09993644100097

0.15304887384201

0.08888430837985

0.18451755431201

$-0.00010695345305$

$-1.22590654443555$

1.21109807313674

$-1.20084828463160$

1.21779596137902

0.00669444199970

2.14550603221887

2.16710646638534

$-0.00143360257312$

$-0.01617520282291$

0.05781262634531

2.15803705622428

$-0.00056621366601$

$-0.05732304754248$

0.22354272494055

$-1.29711569657414$

$-2.23084670364894$

$-1.29138176573505$

$-1.32107688721923$

1.23001977710063

1.30993105522539

2.13146954308472

1.23629304994534

$-0.11675094586238$

0.75625612152141

$-1.02794361948807$

$-0.12454692439427$

1.03063675789924

$-1.09412818243794$

$-1.95462853035798$

$-1.00471654198858$

1.49685096796487

2.40762875078055

1.50640841866070

1.55453563644176

0.26464980448522

$-0.63111863179838$

1.15296919951378

0.30544212987444

$-2.52509085654292$

$-2.51369179633275$

.

$-3.39359561718724$

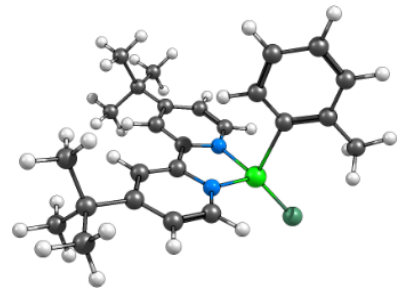

60

Aryl Chloride Triplet 2.4 A Ni-C distance

$\mathrm{Ni}-0.00033327531133 \quad 0.00039997778303$

C $2.51298623249766 \quad 1.23475554280655$

$2.73911109843192-1.08089016748188$

C $3.90183324670844 \quad 1.37642055795075$

C $4.12491175732003-0.99622445123151$

H $2.23195107237742 \quad-2.04178100843601$

C $4.75066208066023 \quad 0.25801347028665$

H $4.32508776147151 \quad 2.37764397191361$

H $4.70484962506387-1.91902794342803$

$\begin{array}{ll}\text { C } 1.53873061716178 & 2.33964854844818\end{array}$

C $1.85309561295889 \quad 3.69991670740185$

C $0.84975521577871 \quad 4.68206654083057$

H $2.89939177101789 \quad 3.99454067557019$

$\begin{array}{ll}\text { C }-0.73026167128453 & 2.85374779554067\end{array}$

$\begin{array}{ll}\text { C }-0.47285353075479 & 4.21810886319793\end{array}$

H $-1.74702338054009 \quad 2.47080827184313$

H $-1.31579952165603 \quad 4.90893613505728$

C -0.89586321861402

0.93102223077594

$-0.81994070547722$

C 0.99642996343343

C 0.11337972104715

H -1.51017125650359

H 1.73150668742226

$\mathrm{Cl}-1.42137747155335$

N 1.93426951067222

N 0.24149186509379

H 1.62817019484840

H 0.14808684070830

C 6.27328893097152

C 1.21096902983854

C 6.60414642385705

H 6.25426470288997

H 6.14046340434353

H 7.69360788731015

C 6.77934785267810

H 6.32367555969867

H 6.55318154309279

H 7.87145093369912

C 7.01508959534309

H 6.84459086895599

H 6.71943262213670

H 8.09945570110735

C 1.92670321601323

H 2.85579538964635

H 1.27882326031925

H 2.19391894419768

C 2.15679216433792

H 1.67735056921534

H 3.09433604262082

H 2.42363916751397

C -0.03527197236564

H -0.73966122886591

$\mathrm{H}-0.57570733609042$

H 0.26786598377133

C -1.91739170637064

H -2.59649689269058

H -1.43808810846514

H $\quad-2.52709063864677$

0.00018076398129

$-0.78714161108814$

0.81902702760450

$-0.72028286446491$

0.87659264325664

0.10028073140735

$-1.32706007034276$

1.52290483955783

$-1.25407191000864$

0.00016752105011

91991800438949

1.43205742845967

0.13086177846902

0.43012341451986

6.17127703310790

1.11021109422782

0.50155109087432

2.10514387879810

1.24218971508391

1.31932023698419

32164746545358

0.86423953163399

1.45080515030379

$-0.91953997373502$

$-1.44305625245078$

$-1.59195590378601$

$-0.75070833895378$

6.41506980476315

5.83058645913980

6.14447766131448

7.47950469662318

6.56166961794242

6.39359773361188

5.98440162571119

7.62883218125500

7.07606957672035

6.86725750948811

6.97199067968520

8.13083702414552

$-1.68539627505681$

$-1.11463978422370$

$-2.43283187061550$

$-2.22054085489056$

0.00015358543248

$-0.28942092930195$

$-0.11135022660404$

0.39716494189791

$-0.21373147368527$

0.00748877720270

36462560806308

$-0.50525822031622$

$-0.17464635049458$

$-0.28871407751395$

0.39557353906163

$-0.36188501418659$

$-0.50402670145422$

$-0.10891039953792$

$-0.21054246183176$

0.01024956226360

$-0.17057534976598$

40015357336634

3.14431360014892

3.03715235170505

4.55180529877515

4.44084654638347

5.19757745545184

5.15070585763721

4.93376093519236

$-1.05499658488674$

$-0.15376760784819$

$-0.15259768147222$

2.45176079219433

6.29140849463794

$-0.48510239972805$

$-0.48177611886351$

$-1.83703085079084$

$-2.68569717179397$

1.92464515224266

$-1.93949882206220$

0.67810899204974

0.66184857379809

1.65517846320830

0.60830015233474

$-0.42434713637258$

0.52996193679322

$-1.24556446146617$

$-0.51495721687240$

$-1.83377172650491$

$-1.92190119142047$

$-2.68238831358838$

$-1.93582458784312$

0.68133946709460

1.65847609765649

0.66445801921644

0.61201458552797

$-0.42024009550982$

$-1.24133416399927$

0.53418818028546

$-0.51046295757589$

2.48408695137284

1.83131861545725

1.83244112853718

3.22902533283277 


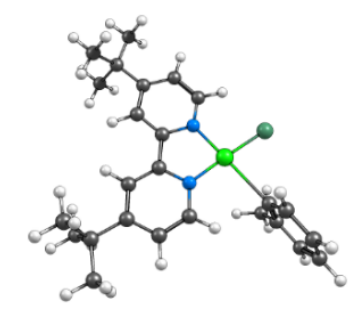

60

Aryl Chloride RKS 2.6 A Ni-C distance

$\mathrm{Ni}-0.00770113267897 \quad 0.05520537153822$

C $-2.37408388205711 \quad 1.44577168565359$

C $-2.75152155587921-0.85760293743260$

C $-3.74888112781657 \quad 1.68699357481028$

C $-4.13047785617331 \quad-0.66137251169919$

H $\quad-2.31044268079458 \quad-1.85440087771482$

C $-4.67861067914255 \quad 0.63364755906665$

H $\quad-4.09799626414217 \quad 2.72166614075435$

H $\quad-4.76676204322600 \quad-1.54673296767020$

C $-1.33578053518646 \quad 2.48232714724680$

C $-1.55817692447162 \quad 3.86055427532408$

C $-0.48405758274071 \quad 4.76769007016597$

H $\quad-2.58584298090329 \quad 4.23036528281285$

C 0.97259085859237

C 0.80389003532536

H 1.95812660789498

H 1.69798967315434

C -0.01350179958355

C 0.12534281543865

C -0.14838576636645

C 0.09043589213079

C -0.17692790294485

C -0.06059799463259

H 0.19407738132103

H -0.28136335002364

Cl 2.14104300786878

N -1.85744875868983

N -0.06727829187728

H -0.22282461810872

H -0.07803982237588

C -6.18766350520227

C -0.74295057052942

C -6.53765534575781

H -6.27711353362370

H -6.00768997733906

H $\quad-7.61843888061369$

C -6.56858267329469

H -6.03918483740374

H -6.33077456138849

H -7.64966441527536

C -7.01990936671654

H -6.83726531912161

H -6.81501529323450

H $\quad-8.09287722490138$

C -1.55144968640571

H -2.52563937202808

H -1.00001659847373

H -1.74633950563419

C -1.55526108016201

H -1.00662150744918

H -2.52957867862152

H -1.75013028133336

C 0.56767600296570

H 1.18152632594028

H 1.17898761436288

H $\quad 0.33741739418089$

C 0.32616497193676

H 1.25219740539922

H -0.49738622414490

2.81942968951109

4.20229195945503

2.34615677840156

4.82669472791733

$-2.54474636933309$

$-3.19652318582801$

$-3.23005376124726$

4.60673275858388

$-4.63787939868382$

$-5.32088391456953$

$-5.15074951381670$

$-5.19011110601179$

$-0.15797667056645$

0.16530457348772

96019316402783

$-2.68992912505833$

$-6.41515492353291$

0.91897052184738

6.28082887165169

1.77875028828682

1.25567815118882

274406662231751

1.99423691956728

1.69227072236625

2.6548730924762

1.10626620057900

1.90710301447855

$-0.37703959692990$

$-1.02317129176171$

$-0.96200129309947$

$-0.12926283297725$

6.71244632016281

6.20249622898690

6.49139321013089

7.79688645435535

6.60608799332866

6.30788236871713

6.09311633070000

7.68922259996473

7.09092107860792

6.92538495933251

6.84958812910293

8.16684078614695

$-2.42371772934538$

$-1.82908188277525$

$-1.70934595880642$

$-3.08727949902580$
0.01332244578519 0.01867323929238 $-0.06284724153208$ 0.01066752798148 $-0.07341711681328$ $-0.08494175360262$ $-0.03619900581071$ 0.04014152322200 $-0.11075389370268$ 0.06396139127244 0.12097972634267 0.15984270729777 0.13649583223060 0.08013889961759 0.13740523166729 0.06124627751129 0.16418628828236 0.00141873360247 $-1.22814364331755$ 1.20326135295173 $-1.21816728657769$ 1.19369832142954 $-0.02166806832368$ $-2.16488422345331$ 2.13394141829666 $-0.03446514919045$ $-0.01733674957037$ 0.04312008324825 2.15234786719487 $-0.04072788991135$ $-0.04496225739874$ 0.22332607132301 $-1.28524394260893$ $-2.21884088980161$ $-1.28292632343880$ $-1.30625469264435$ 1.24221986222929 31894930480655 2.14395829171958 1.25137980660119 $-0.09962642739690$ 0.77388376722213 $-1.01050628998303$ $-0.10449078878676$ $-1.02560539133149$ $-1.08522924542389$ $-1.95303885362221$ $-0.99602675690213$ 1.50167325122200 2.40893466086837 1.51539030565746

1.56263507011024 0.25949330836034 $-0.64025329981659$ 1.14368335807399 0.30483281745249 $-2.50987587062500$ $-2.45500623600764$ 2.67932060038218 $-3.38672751332689$

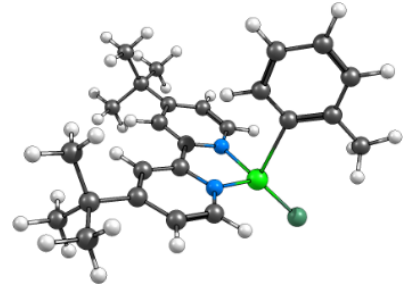

60

Aryl Chloride Triplet 2.6 A Ni-C distance

$\mathrm{Ni}-0.00021978645376 \quad-0.00013033518081$

C $2.51823574713735 \quad 1.23638711028594$

$\begin{array}{ll}\text { C } 2.73490987208914 & -1.08343180623143\end{array}$

C $3.90958579165888 \quad 1.37854600180062$

C $4.12389625585594-0.99838685522527$

H $2.22291723685736-2.04641287613001$

C $4.75608888220771-0.25782761382240$

H $4.33725180131381 \quad 2.38163694637861$

H $4.70108275217847 \quad-1.92301739323863$

C $1.54425181613395-2.34219248196074$

C $1.86096945422215-3.70450517605551$

C $0.85618870363724 \quad 4.68575764439253$

H $2.91001123904900 \quad 4.00210588904217$

C $-0.72947958118301 \quad 2.84995853138691$

C $-0.46972905373462 \quad 4.21712905248171$

H $-1.74936682973862 \quad 2.46364682380802$

H $-1.31403772496335 \quad 4.90639431305856$

C $-0.00006969583450 \quad 0.00053349796149$

C $-0.90118927285451-0.78117714346536$

$\begin{array}{lll}\text { C } & 0.93995388202431 & 0.81683943097047\end{array}$

C -0.82037018353918

C 1.00790156836028

C 0.12097178764858

$\mathrm{H}-1.51253445468887$

H 1.74790646194919

Cl -1.45876529497842

N 1.93235800434565

N 0.24366170120542

H 1.63738368562077

H 0.15895334254857

C 6.28216722933276

C 1.22006645230855

C 6.66308561135284

H 6.34479198394415

H 6.20308316047491

H 7.75562141596203

C 6.74582493909238

H 6.29199461777912

H 6.48334358180106

H 7.83987754691698

C 7.02014928991728

H 6.81403988812187

H 6.75443032075847

H 8.10727238100626

C 1.97587268013987

H 2.90712706675184

H 1.35357278400528

H 2.24609898298580

C 2.13045572947412

H 1.62209340170159

H 3.06842287000830

H 2.39867445304583

C -0.02793907111189

H -0.70727659054978

$\mathrm{H}-0.59669271283064$

H 0.27711149055904

C -1.92691102561183

H -2.59914800504043

H -1.44921033126913

H -2.54192510410158

0.00011320739657 $-0.12920595490962$ $-0.00913317878795$ $-0.18319740895554$ $-0.05572339426561$ 0.06306159591772 $-0.14979017111257$ $-0.24868133018702$ $-0.01848225164109$ $-0.12807807340990$ $-0.18043590507986$ $-0.14479373472989$ $-0.24628022743098$ $-0.00536976599915$ $-0.05035035515398$ 0.06721559548772 $-0.01150013547548$ 2.60011311927436 3.33596191985533 3.21889106766440

4.74369795257889 4.62366644256914 5.38309809468972 5.34688051458450 5.11429429385382 $-0.93297054964088$ $-0.05097105308431$ $-0.04921898990084$ 2.62611027707778 6.47669235054713 $-0.20954362638695$ $-0.20251701089325$ $-1.53017708556814$ $-2.40673277815957$ $-1.60904379115923$ $-1.58879845061222$ 0.99427192967742 0.98738665096826 1.95013489128328 0.96816042979478 $-0.15674158242606$ 0.77662215019319 $-1.00557834553606$ $-0.20283258866694$ $-1.52329235231452$ $-1.60385752904486$ $-2.39972891757133$ $-1.58041766454183$ 1.00110790680508 1.95708576744189 0.99247905486844 0.97649122298517 $-0.14720731894416$ $-0.99577519736936$ 0.78640365459917 $-0.19179225040363$ 2.67227309901711 2.01873048206684 2.02034762018582 3.41475610797924 


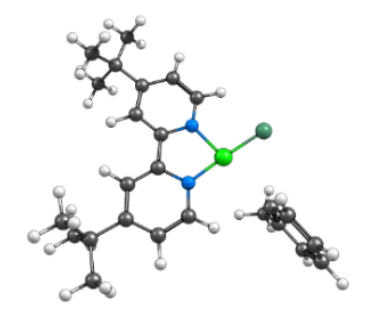

60

Aryl Chloride RKS 2.8 A Ni-C distance

$\mathrm{Ni}-0.00662734502564 \quad 0.11132445583695$

C $-2.37716470609225 \quad 1.48780067184539$

C $-2.74367040469652-0.81890972466228$

C $-3.75306999482194 \quad 1.72227657275186$

C $-4.12366469022095 \quad-0.62881277079481$

H $\quad-2.29535883534303 \quad-1.81256944241498$

C $-4.67741439267983 \quad 0.66379868171177$

H $\quad-4.10756792339623 \quad 2.75512305426750$

H $\quad-4.75613564840590 \quad-1.51701285679449$

C $-1.34187015104974 \quad 2.52792949511777$

C $-1.56789458281861 \quad 3.90531788920580$

C $-0.49651510610371 \quad 4.81602176887497$

H $-2.59673029525863 \quad 4.27170454092560$

C $0.96672945905118 \quad 2.87263178108812$

C $0.79317424662068 \quad 4.25481243526661$

H $1.95369905849542 \quad 2.40287271575307$

H $1.68534161376475 \quad 4.88218023398611$

C $\quad-0.03196248019987 \quad-2.68844330588573$

C $0.16994855629585-3.31284041278286$

C $-0.21556834708677 \quad-3.38559643960807$

C $0.14891555235054 \quad-4.72396277651691$

C $-0.22948381810327 \quad-4.79433395815434$

C $-0.04954266683539-5.45647397750543$

H $0.30072389471441 \quad-5.25330287552085$

H $\quad-0.37243725768299 \quad-5.36198725763682$

Cl $2.13906233665843 \quad-0.11315685771548$

N $-1.85564670816258 \quad 0.20979933151539$

$\begin{array}{lll}N & -0.07108218941207 & 2.01010807930963\end{array}$

H $-0.33989866528819-2.85932264570560$

H $\quad-0.05475075027190 \quad-6.55045240457381$

C $-6.18793520587527 \quad 0.94177532152420$

C $-0.76003092790165 \quad 6.32825771687603$

C $-6.54721932003899 \quad 1.79854217860386$

H $-6.28803484458107 \quad 1.27574547340862$

H $-6.02199172472358 \quad 2.76645211015646$

H $\quad-7.62911497187082 \quad 2.00870941311453$

C $-6.56722835258551 \quad 1.71455987994140$

H $-6.04229767683552 \quad 2.67985441438936$

$\begin{array}{lll}H & -6.32282553305745 & 1.13067033805268\end{array}$

H $-7.64929776581123 \quad 1.92407794823403$

C $-7.01411229862674 \quad-0.35830037154137$

H $-6.82472427784123 \quad-1.00264546934062$

H $-6.81026285447130 \quad-0.94321526340725$

H $\quad-8.08826414524424 \quad-0.11569264728286$

C $-1.57660965405824 \quad 6.75750300835187$

H $-2.54945269084186 \quad 6.24441083142110$

H $-1.02942630653516 \quad 6.53849502744119$

H $-1.77489295527400 \quad 7.84128511523708$

C $-1.56656537344786 \quad 6.65057921031726$

$\mathrm{H}-1.01208368374597 \quad 6.35408466552958$

$\begin{array}{lll}\mathrm{H} & -2.53909076905768 & 6.13436586019694\end{array}$

H $-1.76474315781600 \quad 7.73304095674088$

C $0.54811910514692 \quad 7.14262369158485$

H $1.15773767692521 \quad 6.97918972977082$

H $1.16487556107943 \quad 6.90327452750745$

H $\quad 0.31457839954887 \quad 8.21777355457894$

C $0.41988484167948 \quad-2.51509829414769$

H $1.33452741751129 \quad-1.91050975193542$

H $-0.40413898191943 \quad-1.8070108312736$

H 0.52887346527438

$-3.16146958723449$
$-0.02164034320079$

0.01989688135138

$-0.05908141405939$

0.01714062983605

$-0.06387349526522$

$-0.08265529964038$

$-0.02518308553332$

0.04705558375926

$-0.09792293094947$

0.06052890848842

0.11913665982728

0.15276160353812

0.13997160358852

0.06542079055782

0.12371835599513

0.04145924548312

0.14604021425896

0.00402279354473

$-1.22843116467314$

1.18987224373156

$-1.24001280856243$

1.15761291236340

$-0.06144781447288$

18866613373905

2.08361197696356

$-0.05562145140140$

$-0.01748597789382$

0.03343876541079

2.14160304797533

$-0.09723906932078$

$-0.02790221652151$

0.21794957438480

$-1.26762285733339$

$-2.20174925903286$

$-1.26852424293796$

$-1.28434074027432$

1.26007444121220

1.33362452233451

2.16142140204107

1.27349956065109

$-0.07776187541646$

0.79563099859355

$-0.98889943373674$

$-0.07836373540022$

$-1.02654731574529$

$-1.08110043179668$

$-1.95697747482132$

$-0.99564194680676$

150069445096260

2.40495644981849

1.51954295746884

1.56287594825920

0.24726855181979

$-0.65573667704407$

1.12821209986721

0.29391554048715

$-2.48587578676405$

$-2.37731728342880$

$-2.67735557361207$

$-3.37090442518940$

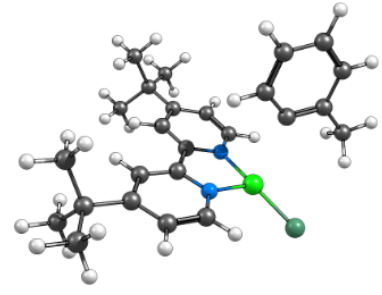

60

Aryl Chloride Triplet $2.8 \AA \mathrm{Ai}-\mathrm{C}$ distance

$\mathrm{Ni}-0.23339825295833 \quad 0.31672933413110$

C $2.34793557386758 \quad 1.38957362758977$

C $2.42424387060095 \quad-0.93439012361820$

$\begin{array}{ll}\text { C } 3.74503372113968 & 1.45004798822525\end{array}$

C $3.81596179813310-0.93149091168077$

H $1.85987884442203 \quad-1.86707862359288$

$4.52506731188869 \quad 0.28341027110357$

$\begin{array}{lll}\text { H } 4.23084025699005 & 2.42714453814728\end{array}$

H $4.33550230506425-1.88844322717410$

C $1.44538690485343 \quad 255344175159496$

C $1.83908493205219 \quad 3.88800155500941$

C $0.89017525894823 \quad 4.92564028049674$

H $2.90168106092656 \quad 4.11991908605922$

C $-0.79780318563861 \quad 3.19580748263337$

C -0.45916062491546

H -1.83637165085610

H -1.26188803966610

0.04760428065433

C -0.42382478066778

0.63131896437956

$-0.28599642143763$

C 0.76008487449486

C 0.29763604862006

H -0.64746228612970

H 1.21368050893473

$\mathrm{Cl}-2.18857756167897$

N 1.68190292379901

N 0.12392978849624

H 0.98824210595053

H 0.38760786613582

C 6.05941503629879

C 1.33804834081130

C 6.53239532143830

H 6.21495297260998

H 6.13474674655880

H 7.63228589877557

C 6.52558017251570

H 6.13025903045328

H 6.19878511590850

H 7.62563659709786

C 6.71447584495446

H 6.44418888928144

H 6.44034168701986

H 7.81065131919757

C 2.08987596298790

H 2.98577033649399

H 1.44233348506970

H 2.41847867504968

C 2.28472851375597

H 1.77892533367128

H 3.18666199160863

H 2.61532775291211

C 0.14506385233706

H -0.55455338240889

$\mathrm{H}-0.41863322663411$

H 0.51011292297228

C -1.05033661993656

H -1.85016324184354

H -0.30842314750713

H -1.47045543285360

4.54165313324498

2.86610721394867

5.28002092217764

$-0.07846333729126$

$-1.26930111876788$

0.93104609645760

$-1.41652809284375$

0.76590208840265

$-0.41238146159567$

$-2.33549764904838$

1.55587722075782

$-0.35858598522227$

0.19163034740721

2.21226250013450

1.85421198174102

$-0.55262127128879$

0.36687991339691

6.38762214694195

1.05824674348665

0.49193895004074

2.08079094994817

1.12813115323845

1.19581481172988

2.22336976873197

0.73160181718546

1.26390175082054

$-1.02608445665272$

$-1.55964657129071$

$-1.66065678929927$

$-0.92137416493127$

6.54879785833289

5.91079547174436

6.28890355006062

7.59297746040705

6.75901624697380

6.65112393433120

6.12766194706388

7.80595098561244

7.36337187440315

7.17171820597873

7.31750967075040

8.39612410596760

$-2.35884311282704$

$-1.95960534446841$

$-2.81828977234936$

$-3.16347238946739$

0.02980916851563

$-0.09583732301939$

0.06179349076132

0.10245612400073

0.05231954754645

0.13718227341958

0.03096719540341

0.15574617419137

0.11549319775323

$-0.14935461660494$

$-0.29346756178928$

$-0.31116270873763$

$-0.39607458408230$

0.05521655756640

$-0.18249277207213$

0.03206373428872

$-0.18349897280011$

2.78750028860161

3.34609644579490

3.53899297293963

4.74348638621671

4.93213122975155

5.52795667634298

5.22149616479036

5.54131009839728

$-0.56761921247504$ $-0.01452687583871$ .03696196221555 3.06763142813237 6.60984144105327 $-0.03366836796526$ $-0.46775122222377$ $-1.33662796129554$ $-2.22645276714439$ $-1.43070478846275$ $-1.35258911516402$ 1.18932959354743 1.16756406285401 2.13297343000830 1.20559900306094 0.04380079900886 0.96902298037446 $-0.81428466750291$ 0.03576722176743

$-1.81263174065213$

$-1.86566759355670$

$-2.66481664646048$

$-1.94124719282084$

0.70086397823886

1.67327134093915

0.72023653107678

0.60386069564891

$-0.45668172721497$

$-1.28592680151705$

0.48883600170297

$-0.56956735111391$

2.50515181775003

1.86315586867779 


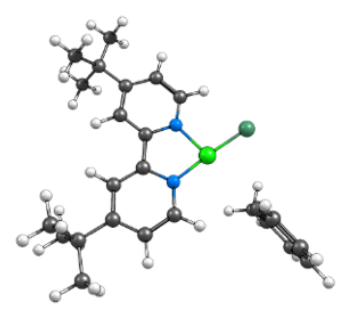

60

Aryl Chloride RKS 3.0 A Ni-C distance

$\mathrm{Ni}-0.00053890017017-0.00035490097041$

C $-2.40522862362958 \quad 1.31198629538896$

C $-2.71026434576332 \quad-1.00270530322071$

C $-3.78639495496829 \quad 1.51121712871430$

C $-4.09440422651761 \quad-0.84746865395024$

H $\quad-2.23372761578673 \quad-1.98311193725512$

C $-4.68172104218000 \quad 0.42986139889393$

H $-4.16838305358899 \quad 2.53381332120895$

H $-4.70322192617393 \quad-1.75052151554081$

C $-1.39661029567407 \quad 2.37646944360363$

C $-1.65690777487789 \quad 3.74675160944162$

C $-0.60900147029658 \quad 4.68274124428418$

H $\quad-2.69475133779749 \quad 4.08737161076728$

C $0.90346219498387 \quad 2.77735526180657$

C $0.69448458423809 \quad 4.15360011364232$

H $1.90209717297316 \quad 2.33294093122101$

H $1.57046530149370 \quad 4.80204684077325$

C $\quad-0.00082103157137 \quad-3.00035319511116$

C $0.31299130344723 \quad-3.57801282400207$

C $-0.26240714201741 \quad-3.73022252847092$

C $0.32691025277117 \quad-4.98887954215049$

C $-0.24011442095790 \quad-5.13824815107652$

C $0.05276324661763 \quad-5.75917614020731$

H $0.56576832135518 \quad-5.48743157102742$

H $\quad-0.44375135007132 \quad-5.73628124206229$

Cl 2.14639426363309 -0.19191946529427

N $-1.85107895429131 \quad 0.04854650628006$

$\begin{array}{lll}\mathrm{N} & -0.11259433403914 & 1.89046933311961\end{array}$

H $\quad-0.47477403464797 \quad-3.23471856573486$

H $0.07670057447400 \quad-6.85148365603410$

C $-6.19870598706274 \quad 0.66896323499938$

C $-0.91065283396444 \quad 6.18669412238427$

C $-6.55832504425926 \quad 1.53835843115253$

H $-6.26926189577064 \quad 1.03931755931477$

H $-6.05864428124712 \quad 2.51937482175740$

$\begin{array}{lll}\text { H } & -7.64486484575427 & 1.72084441790127\end{array}$

C $-6.62033704307250 \quad 1.40856521947864$

H $-6.12240344655664 \quad 2.38573521150527$

H $-6.37622808812443 \quad 0.81531067854835$

H $\quad-7.70760945341490 \quad 1.58957744050553$

C $-6.98988589886349-0.65073574833100$

H $-6.79919989049215-1.30552758968895$

H $-6.75517639802079-1.21393660027753$

H $\quad-8.06977780540152 \quad-0.43570170297157$

C $-1.71771035346242 \quad 6.61763798514689$

H $-2.67656979922485 \quad 6.08193047822700$

H $-1.15053891304122 \quad 6.42855887971502$

H $-1.94326687137880 \quad 7.69551239609460$

C $-1.74518593784334 \quad 6.46682796124111$

H $-1.19818974212810 \quad 6.16783931135497$

H $-2.70504557797452 \quad 5.92717268519246$

H $-1.97061573720272 \quad 7.54293498506086$

C $0.37642111595759 \quad 7.03199848796451$

H $1.00420287734715 \quad 6.89934171467312$

H $0.98476425541530-6.79242290338457$

H $0.11572524634562 \quad 8.10014499513538$

C $0.64069896010306 \quad-2.73582536862322$

H $1.52578156606985-2.11097716567511$

$\begin{array}{lll}\mathrm{H} & -0.18530087674957 & -2.04332470963478\end{array}$

H 0.83684905780583
$-3.35164145157270$
0.00237186298748 0.02280342063902 $-0.10464061394748$ 0.00132483471142 $-0.13172140400797$ $-0.13766012829969$ $-0.07961755174112$ 0.04039545058057 $-0.19389994457738$ 0.10003973432094 0.17872280352854 0.24556764565992 0.18914629131831 0.14918094666719 0.22810823307923 0.13371471771587 0.27562908875712 $-0.00081609105138$ $-1.23079459712940$ 1.14800944234412 $-1.28219032509111$ 1.07543954124860 $-0.14332727354466$ 2.22972744089504 1.97058085102263 0.00378859330478 $-0.02707868157313$ 0.08512549916017 2.10098455831561 $-0.20955869649859$ $-0.10469846675324$ 0.33223852743265 $-1.33549021977534$ $-2.27382688091559$ 1.30995171862974 $-1.36799924564888$ 1.18962703615537 1.28914811884310 2.08491304962950 1.18752646645450 $-0.19201749721242$ 0.67328085413772 $-1.10938692338166$ $-0.20761586648569$ $-0.91787916761914$ $-0.99713395094729$ $-1.84288625402795$ $-0.87154882211976$ 1.60699609489976 2.51498313855186 1.60093408015451 1.68459222542197 0.39684358615043 $-0.49874447035370$ 1.28355665974771 0.45803975737642 $-2.44067734021337$ $-2.24121720854679$

. 67530633769738 $-3.33240983420929$

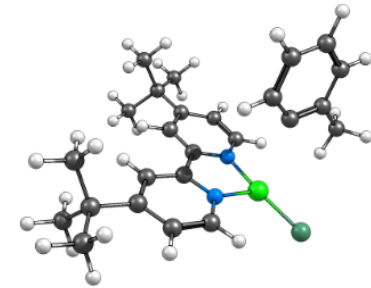

60

Aryl Chloride Triplet 3.0 A Ni-C distance

$\mathrm{Ni}-0.30654256249307 \quad 0.32426947963475$

C $2.28667169066254 \quad 1.36524398706220$

C $2.32940525954338-0.96125167165738$

$\begin{array}{ll}\text { C } 3.68333163912174 & 1.40723950257852\end{array}$

C $3.72145016959098-0.97621862354421$

H $1.75277842377944 \quad-1.88843051201726$

C $4.44766859694098 \quad 0.22945510435530$

H $4.18273763847227 \quad 2.37816273594398$

H $4.22698670013327-1.94101235695309$

C $1.40016974251561 \quad 254104462627337$

C $1.81343128893477 \quad 3.87347373598046$

C $0.87689581920707 \quad 4.92222141219178$

H $2.88214023304717 \quad 4.09610417338970$

C $-0.83796451094528 \quad 3.20530400201571$

C $-0.48052882783387 \quad 4.54993667244389$

H $\quad-1.88305439991227 \quad 2.88603453710729$

H $-1.27522293591495 \quad 5.29660534623470$

C 0.030629058

C -0.17221416120251

C 0.37476111576979

C -0.00316873807393

C 0.53704476873734

C 0.34678777226051

H -0.15421385924252

H 0.80626136061880

Cl -2.23314334616231

N 1.60205385149242

N 0.07164096349418

H 0.51763403214027

H 0.46859349141769

C 5.98260165122551

C 1.34659090027479

C 6.48793472667830

H 6.17960009419101

H 6.10495712128162

H 7.58874831648346

C 6.43702359106152

H 6.05464510116076

H 6.08758610177931

H 7.53737783257526

C 6.61844079837124

H 6.32312727875255

H 6.35318287187299

H 7.71581459027202

C 2.16640572259805

H 3.05722690057101

H 1.55973371943193

H 2.51176796551693

C 2.23735998455309

H 1.68224452309630

H 3.13030873518515

H 2.58361664475997

C 0.16426908518183

H -0.49485328006768

$\mathrm{H}-0.44648386394491$

H 0.54455404085180

C -0.55909354715347

H -0.64025583066622

H 0.17647352515094

H -1.53913243103860

0.09715109722025

$-1.19067440283602$

1.18051919492321

$-1.37254943837142$

0.97813455131130

$-0.30297742621667$

$-2.36978792832579$

1.81693840834198

$-0.43187442822788$

0.17568616714522

2.21067255291052

2.17939696375645

$-0.46960533169278$

0.29338671839914

6.38215584940634

0.99078197533108

0.43688861351883

2.01914805541973

1.04683525326138

1.10484482205348

13733398951406

0.63598879420134

1.15918694407050

$-1.10851527275036$

$-1.64765257544732$

$-1.73096911223454$

$-1.01786091033249$

6.56422990910514

5.91737024438539

6.33023068672290

7.60715905086566

6.71797800932568

6.59575128908355

6.07574301129912

7.76277203424872

7.37048092442306

7.20444231280352

7.31019479139683

8.40143474258741

$-2.34305968858741$

$-1.99735891785407$

$-3.16475219008685$

$-2.76545915210825$

$-0.11818174730212$

$-0.13404719117021$

$-0.01057726826229$

0.11745601628568

0.00758054611847

0.03535060739238

04516181526184

$-0.15635293077841$

0.06591376055834

$-0.19755577169338$

$-0.29185412987574$

$-0.33366494523330$

$-0.33398185457278$

$-0.19761176279466$

$-0.28043520582903$

$-0.16623926065746$

$-0.30573587103980$

2.85414604964437

3.34361438672952 3.64928871627441

4.73551096326342 5.03254889755344 5.56794750855571

16790099736049 5.68416758457215

$-0.68302338996356$ $-0.07703735511230$ 15510974210886 3.22200597273730 6.64287501884717 01948966357185 $-0.43518659973128$ $-1.30702779350207$ $-2.20780567032666$ 1.39854154168016 $-1.30245960294471$ 1.21954295175447 20074729026896 2.15271015707740 1.25602789965948 0.05645858834276 0.97075424590454 $-0.81322150215992$ 0.07087318132021 $-1.73697216646469$ $-1.75941495233338$ $-2.62595975206280$ $-1.82580917224210$ 0.78693188800558 1.73031663112420 0.83795460175889 0.72910299681520 $-0.46194655569608$ $-1.32904866155710$ 0.45305765480116 $-0.53352848276019$ 2.44307201259200 1.40262632896746 2.48969382072122 2.71954554625357 


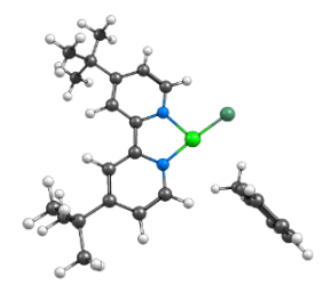

60

Aryl Chloride RKS $3.2 \AA$ Ni-C distance

$\mathrm{Ni}-0.02966723908317-0.06228859722122$

C $-2.48565865207225 \quad 1.14725769034812$

C $-2.69452832086321-1.17792342760763$

C $-3.87387033521965 \quad 1.28926593965068$

C $-4.08397889030588-1.07954674365756$

H $\quad-2.17564411395884-2.13651128745845$

C $-4.72336746064478 \quad 0.17195754035860$

H $\quad-4.29824198591277 \quad 2.29461679161069$

H $\quad-4.65512640599281 \quad-2.00632700524857$

$\begin{array}{lll}\text { C } & -1.52035540289928 & 2.25093187934497\end{array}$

C $-1.83443719970694 \quad 3.60869318832633$

C $-0.82437926952524 \quad 4.58515349857608$

H $\quad-2.88502646419738 \quad 3.90731271125024$

C $0.76319554738997 \quad 2.74345114902550$

C $0.49928051752826 \quad 4.10919251787949$

H $1.77879842299587 \quad 2.34021428062592$

H $1.34865508014563 \quad 4.79196495289949$

C $0.02831951057755-3.26176290734091$

C $0.40940094590461 \quad-3.78825317354695$

C $-0.26103002681338-4.02949120760986$

C $0.46661891166919 \quad-5.19637786719951$

C $-0.19521531579132 \quad-5.43411078377188$

C $0.16696084638193 \quad-6.00804854840715$

H $0.75914061106677 \quad-5.65927512711533$

H $\quad-0.42032658372590 \quad-6.06531450462343$

Cl $2.11827875986026 \quad-0.19175076191128$

N $-1.88045005406950 \quad-0.09162812523153$

N $-0.21750727398503 \quad 1.81752327840768$

H $\quad-0.52809852912914-3.57029028110495$

H $\quad 0.22508541391758 \quad-7.09673648617337$

C $\quad-6.24908813332774 \quad 0.34797521661191$

C $-1.18553538350415 \quad 6.07477979176419$

C $-6.64686032559229 \quad 1.21249139434573$

H -6.339250301082390 .73411784271942$

$\begin{array}{lll}\mathrm{H} & -6.18823548038468 & 2.21313907478716\end{array}$

H $\quad-7.74008924511838 \quad 1.35003577988728$

C $-6.69858479720175 \quad 1.05793339456037$

$\begin{array}{lll}\mathrm{H} & -6.24207440555913 & 2.05432057700067\end{array}$

$\begin{array}{lll}H & -6.42788528299548 & 0.46772410787587\end{array}$

H $\quad-7.79250159696247 \quad 1.19302559847660$

C $-6.98486978423861-1.00268038330196$

H $-6.76492502357837-1.65669713052674$

H $\quad-6.72930109124780 \quad-1.54741833120304$

H $\quad-8.07280085555641 \quad-0.83263299648707$

C $-2.01634581971680 \quad 6.48645100949049$

H $-2.95336452325906 \quad 5.91353237298233$

H $-1.44729917616728 \quad 6.33050671608082$

H $-2.28475399860464 \quad 7.55379867849368$

C $-2.02325750907487 \quad 6.30744401272625$

H $-1.45960620769717 \quad 6.02066625135823$

H $-2.96089031409759 \quad 5.73005881289250$

H $-2.29094950006443 \quad 7.37277568696685$

C $0.06700131381961 \quad 6.97031281718461$

H $0.69448310911501 \quad 6.87252623050323$

H $0.68945859240392 \quad 6.74598419764067$

$\begin{array}{lll}\text { H } & -0.23592602369177 & 8.02641785007359\end{array}$

C $0.76085661207037 \quad-2.89783595681143$

H $1.62258365847055 \quad-2.25948894686277$

H $\quad-0.07257890313193 \quad-2.21591063595442$

H $1.00238950943481-3.47787557934932$
$-0.02929559348089$

0.00810447418419

$-0.14126795509181$

0.01226800381691

$-0.16402878810646$

$-0.18483351182174$

$-0.09866690496643$

0.03867994925721

$-0.23379136545683$

0.09280608156559

0.18796023228499

0.25937427106214

0.20818106274333

0.13541059245239

0.22944347177059

0.11116772938142

0.27918263832770

0.02796442534279

$-1.26068636776737$

1.08743309507535

1.35296976044041

0.97347712177524

$-0.24966139981324$

$-2.30367951572965$

1.84040041155008

$-0.03438625785606$

$-0.05455535830305$

0.06695802357247

2.04524581168437

$-0.34714873629900$

$-0.11906388827290$

0.36410648543590

$-1.34152960310886$

$-2.28471720097152$

$-1.30813166563374$

$-1.37064213326062$

1.18245232015978

1.28967830782030

2.07208510417745

1.18381254368590

$-0.21674534103716$

0.64216202458760

$-1.13966315166892$

$-0.22811902880012$

0.87692175866761

$-0.95716499624872$

$-1.80694455954012$

$-0.81740199484094$

1.64630437213123

2.54801730787402

1.63932851495273

1.73702686560357

0.43112809832021

$-0.46914550156953$

1.31201613122709

0.50527469506739

$-2.42893050369406$

$-2.17706247176900$

2.66779752846414

$-3.33342366088961$

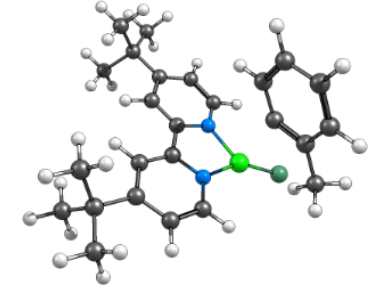

60

Aryl Chloride Triplet $3.2 \AA \mathrm{Ni}-\mathrm{C}$ distance

$\mathrm{Ni}-0.00005425733343 \quad 0.00014639961367$

C $2.51124187063200 \quad 1.22593722527406$

C $2.71328450437449-1.07077027010542$

$\begin{array}{ll}3.90010330456494 & 1.37461233815992\end{array}$

C $4.10303584578800-0.97984535343981$

H $2.19093021632313 \quad-2.02306230825949$

C $4.74057737445095 \quad 0.26641019727967$

$\begin{array}{lll}\text { H } & 4.33285473465289 & 2.36989317673712\end{array}$

H $4.67546232468588-1.89295968338359$

C $1.53453499452331 \quad 2.30762052120006$

$1.85064939283218 \quad 3.65368823984073$

C $0.84674602604160 \quad 4.61723127653900$

H $2.90090335002662 \quad 3.95481963063227$

C $-0.73852717699246 \quad 2.78791423466882$

C $-0.47842062689809 \quad 4.14053619566074$

H $-1.76155325045451 \quad 2.40359340695257$

$\begin{array}{lll}H & -1.32482817904116 & 4.81263587128760\end{array}$

$\begin{array}{ll}0.00010135343780 & 0.00000197746832\end{array}$

$\begin{array}{lll}\text { C }-1.31433284941974 & 0.03779437526068\end{array}$

C $1.11528606075711-0.00249521422326$

C $-1.48980519414666 \quad 0.08522311379907$

$\begin{array}{lll}\text { C } & 0.91516396176272 & 0.04391782872956\end{array}$

$\begin{array}{lll}\text { C } & -0.39049799728877 & 0.08858006329859\end{array}$

$\begin{array}{lll}\text { H } & -2.50640442263968 & 0.11557622838803\end{array}$

$\begin{array}{lll}\text { H } & 1.77455310960702 & 0.04205877207888\end{array}$

$\mathrm{Cl}-1.16225736968858 \quad-1.65850455117383$

N $1.92019555007335 \quad 0.00014800669005$

N $0.23270645519628 \quad 1.86579922442754$

H $2.13066227881084-0.04158284878602$

H $\quad-0.55406254822016 \quad 0.12313956340008$

C 6.26610947621015

C $1.21248483377730 \quad 6.09223126970839$

C $6.62485056789746 \quad 1.41225395488430$

H $6.28463614660838 \quad 1.01706482721432$

H $6.17021335012357 \quad 2.40601111940014$

H $7.71687043242882 \quad 1.55195256190890$

C $6.75923946752600 \quad 1.04039669077344$

H 6.30795625987289

H $6.51728086700749 \quad 0.37491184157561$

H $7.85282703109415 \quad 1.17615436440093$

C $6.99744067575181 \quad-0.89039185632573$

H $6.80560345640667-1.61688461171685$

H $6.71204615358196 \quad-1.35358444748502$

H $8.08449179287115 \quad-0.71730548104867$

C $1.99036371941745 \quad 6.62145506345287$

H $2.92205693018622 \quad 6.06063477467824$

H $1.38231465859708 \quad 6.55217859930613$

H $2.26318314290944 \quad 7.67914750969811$

C $2.10294886225384 \quad 6.20821330104968$

H $1.57838957610029 \quad 5.83393862791580$

H $3.03965537391469 \quad 5.63809542846765$

H $2.37352935837938 \quad 7.26163969075307$

C $-0.03604340512877 \quad 6.97290961148726$

H $-0.70119652277142 \quad 6.95629208034579$

H $-0.62017392808342 \quad 6.66552034344488$

H $0.26943382169612 \quad 8.01895158439566$

$\begin{array}{lll}\text { C } & -2.49294577945819 & 0.01874228463116\end{array}$

$\begin{array}{lll}\text { H } & -3.16695198192637 & 0.87662249890543\end{array}$

H $-2.13561107907426 \quad 0.03808510445606$

H -3.09276903958773
0.00003391203456 0.10896718256125 25690651268584 0.05412221535019 0.31362852935321 $-0.38161862406695$ 15822676729223 0.17959077972989 $-0.48192775196823$ 0.32808261939946 0.52629853379699 0.73008474486521 0.52407012936891

0.51211597974179 0.71514585860881 0.50176196293553 0.86274765197702 3.20003390373280

3.65259131879683 4.02151548020559

5.05500634736818 5.41441597089857 5.92198974432542 5.46679805343663 6.09397513531893 $-0.69690215074242$ $-0.04909767111610$ 0.32588866953133 3.61207668141166 7.00361114507540 $-0.21349446433827$ 0.95614596755111 $-1.36934379443281$ $-2.33950454126054$ $-1.23428164899777$ $-1.42307807205858$ 1.12902300909438 1.33730951782996 1.97262663718014 1.10586524018003 $-0.45170131478998$ 0.35408634685616 $-1.40976352478962$ $-0.48355757118451$ $-0.27449400206859$ $-0.44777407544249$ $-1.19032752824313$ $-0.12653220889647$ 2.21857356602592 3.11160780908170 2.11785708793579 2.39794457782866 1.15855770290728 0.28023671409624 2.04069533153758 1.31722509775604 2.70536638134371 2.86991704337478 1.66566285611816 2.81979104355909 


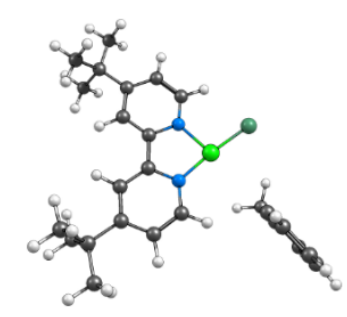

60

Aryl Chloride RKS $3.4 \AA \mathrm{Ai}-\mathrm{C}$ distance

$\mathrm{Ni}-0.00017893471236 \quad-0.00024475468956$

C $-2.46768238949029 \quad 1.18487287376308$

C $-2.65362848133260-1.14447987830255$

C $-3.85708329067306 \quad 1.31336093490541$

C $-4.04395359659154-1.05942332426018$

H $\quad-2.12414169004662 \quad-2.09813623140883$

C $-4.69527957969178 \quad 0.18663714149686$

H $\quad-4.29154718516877 \quad 2.31482063513266$

H $\quad-4.60621204976288-1.99225481273665$

C $-1.51178438113796 \quad 2.29756592468407$

C $-1.83644060177921 \quad 3.65372585440080$

C $-0.83427143508826 \quad 4.63839791703680$

H $\quad-2.88939528084649 \quad 3.94456631202044$

$\begin{array}{lll}\text { C } & 0.76858528148268 & 2.80820174756257\end{array}$

C $0.49330550084797 \quad 4.17253661362427$

H $1.78753702047606 \quad 2.41340380968675$

H $1.33689622112073 \quad 4.86235524360892$

C $-0.00006143329882-3.40024421833917$

C $0.40674460627790 \quad-3.86820090930321$

C $\quad-0.32681874685589-4.21530788804334$

C $0.45276300556166 \quad-5.27100734707226$

C $-0.27229686747656 \quad-5.61395810146134$

C $0.11612245498086-6.13144116961559$

H $\quad 0.76539056226425 \quad-5.69099407830659$

H $\quad-0.52744915579569-6.28436597657168$

Cl 2.14357998093372 -0.14002450607777

N $\quad-1.85093738601399 \quad-0.04847970734164$

$\begin{array}{lll}\mathrm{N} & -0.20511757395055 & 1.87459832066649\end{array}$

H $-0.61465466803486-3.80166929752528$

H $0.16589982394616 \quad-7.21472662520168$

C $-6.22264606360656 \quad 0.34739090155999$

C $-1.20753120891140 \quad 6.12616364261164$

C $-6.62734941883466 \quad 1.19757876961902$

H $-6.31369330114667 \quad 0.71439012450617$

H -6.178914877154

H $\quad-7.72185414172585$

C -6.68070180434105

H -6.23384674738268

H -6.40568465563897

H $\quad-7.77584951205249$

C -6.94493876244450

H -6.72038331265671

H -6.68215414171469

H -8.03445023034234

C -2.03000208826021

H -2.96194336636383

H -1.45130850007351

H -2.30687989065832

C -2.05866251322676

H -1.50108312644308

H -2.99178804500510

H -2.33527783246375

C 0.03756767443613

H 0.67382820337529

H 0.65378214361593

H $\quad-0.27400725149862$

C 0.79468929003166

H 1.65024808576644

H -0.03001348426271

2.20307564408617

1.32386094996293

1.06387171310933

2.06543222326748

0.48368308147348

1.18853823275905

$-1.01123857773635$

$-1.65558891101490$

$-1.56144278025828$

$-0.85195812663520$

6.51959831222599

5.93888046445119

6.35905964312357

7.58540218577545

6.36464708554112

6.09096839791254

5.78004890606887

7.42869674508986

7.03178935399035

6.93049915374148

6.82058691291768

8.08618067281464

$-2.92387533139729$

$-2.30134732926435$

$-2.22755470525807$

$-3.46004582037538$

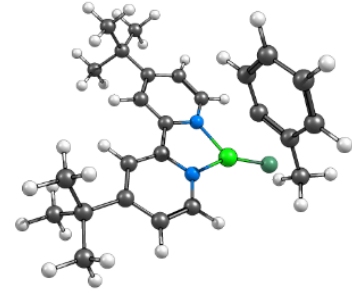

60

Aryl Chloride Triplet $3.4 \AA \mathrm{Ni}-\mathrm{C}$ distance

Ni $-0.00161283009671 \quad 0.00025304921980$

C $2.50344283571986 \quad 1.21866697197054$

C $2.71685666457783-1.06592491282778$

C $3.89347261097800 \quad 1.36349884076922$

C $4.10795613763922-0.97814475064710$

H $2.19860601774561 \quad-2.01197688427486$

C $4.74054758266294 \quad 0.25959132009392$

$\begin{array}{lll}\text { H } 4.32203147693278 & 2.35211896814621\end{array}$

H $4.68553833877428-1.88718603988601$

C $1.51871012965708 \quad 2.29642694059733$

C $1.82429616751005 \quad 3.64046231385493$

C $0.81160949153777 \quad 4.60136457476008$

H $2.87352002581641 \quad 3.94268820775987$

C $-0.76136208851018 \quad 2.77293104917971$

C $-0.51128038892267 \quad 4.12358437851287$

H $\quad-1.78251538216714 \quad 2.38764472506864$

H $-1.36389264356038 \quad 4.79381147520775$

C $0.00411121394320 \quad-0.00313365107224$

$\begin{array}{lll}\text { C } & -1.33778159500931 & 0.05315909233220\end{array}$

C $1.06573633764862 \quad-0.00511728290399$

C $-1.60406002782482 \quad 0.12226842994923$

$\begin{array}{lll}\text { C } 0.77221306358332 & 0.06294713399495\end{array}$

C $-0.56426929136518 \quad 0.12735172078716$

H $-2.64527382408491 \quad 0.16878690168370$

$\begin{array}{lll}\text { H } 1.58397160959830 & 0.06300590546029\end{array}$

Cl $-1.15999524583745 \quad-1.66784428649163$

N $1.91695160612997 \quad 0.00056536713104$

N $0.21797615799245 \quad 1.85209860230874$

H $2.10464172040359 \quad-0.05950497013217$

H $\quad-0.79930089954968 \quad 0.17928762842508$

C $6.26742646609057 \quad 0.43403646881103$

C $1.16578324779211 \quad 6.07432967887296$

C $6.68302050040579 \quad 1.43885580860036$

H $6.38769541465674 \quad 1.07896989631927$

H $6.22514675613522 \quad 2.42926979837057$

H $7.77675091702716 \quad 1.57565141415810$

$\begin{array}{lll}\text { C } 6.69803812050744 & 0.98069394331579\end{array}$

H $6.23849677627728 \quad 1.95657861284827$

$\begin{array}{lll}\text { H } & 6.41609344735244 & 0.28680437265063\end{array}$

H $7.79168901870394 \quad 1.11415942769120$

C $7.00605831917283-0.89548082124186$

H $6.77427557471247-1.64855394000580$

H $6.76523303942623-1.32414992724266$

H $8.09384776223273 \quad-0.72592229902396$

C $1.99675419416083 \quad 6.61603210644994$

H $2.93619987334634 \quad 6.05807552233225$

H $1.43014309980256 \quad 6.55442747495942$

H $226102744954690 \quad 7.67266001880897$

C $1.99953707015614 \quad 6.17882038934526$

H $1.43611750398298 \quad 5.79639823844475$

H $2.93974969260469 \quad 5.60922200006229$

H 2.26182840303148 7.23049248009065

C $-0.09128454569994 \quad 6.95245352345441$

H $-0.71719519572905 \quad 6.94292702993689$

H $-0.71332462846095 \quad 6.63700291967064$

H $0.20581682956930 \quad 7.99728923042556$

$\begin{array}{lll}\text { C } & -2.44787011453185 & 0.02876766185253\end{array}$

H $\quad-3.14650089068659 \quad 0.87216828039370$

H $-2.02118279752340 \quad 0.06935175345413$

H -3.03600200398527
0.00053141904558

0.23283101473594

$-0.19724837715637$

0.24943383873479

$-0.18777360363351$

$-0.37562028664708$

0.03967879857634

0.43033990562977

$-0.36064371581610$

0.43263311949357

0.65704786400484

0.82916401787602

0.69790495585345

0.52717521957368

0.75362753030722

0.46950138920536

0.87132792562629

3.40052491603511

3.75548569511074

4.28768980775304

5.14303513031831 5.66339302107386 6.08073550633684 5.48562672538058 6.39894854844395 $-0.67339945150062$ 0.00839678750901 0.37403998842016 3.94515416964547 7.14830566978812 0.06281110499417 1.08475742680308 $-1.04059496622766$ $-2.03879841442571$ $-0.89250525921899$ $-1.03814338116143$ 1.44680489281817 .66803245947840 2.25435112148905 1.47956532294879 $-0.18660610954178$ 0.58348675946120 $-1.17257846042517$ $-0.16091257388687$ $-0.10507897199763$ $-0.24189100170834$ $-1.04764250366372$ 0.06457918889615 2.38627931619225 3.25178416225948 2.32168266077284 2.58721230264583 1.23998481823834 0.33317260498317 2.09288898742557 1.42104983615452 2.72963026720054 2.86041858776194 1.71683520121639 2.78938311879302 


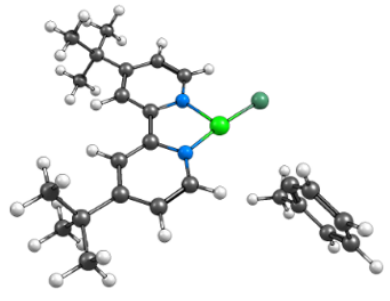

60

Aryl Chloride RKS 3.6 A Ni-C distance

$\mathrm{Ni}-0.00007248830926-0.00018142256916$

C $-2.50491137499401 \quad 1.10516674783016$

C $-2.61422754968785-1.23180571251961$

C $-3.89774101770213 \quad 1.18766810847804$

C $-4.00667448602127-1.19248392258418$

H $\quad-2.05204349543135 \quad-2.16753285098600$

C $-4.69810405495508 \quad 0.03248277129606$

H $-4.36522076389975 \quad 2.17463084773266$

H $-4.53835913655155 \quad-2.14396234959101$

C $-1.58390889766433 \quad 2.24802395604488$

C $-1.94906859656047 \quad 3.59463263837104$

C $-0.97687785633307 \quad 4.60944391674036$

H $\quad-3.01029806256481 \quad 3.85387554402689$

C 0.68081468784873

C 0.36423735028732

H 1.71134146050100

H 1.18637867076020

C -0.00018837291810

C 0.38460130794399

C -0.29948953603010

C 0.44084865707157

C -0.23599261629048

C 0.13302416593943

H 0.73837799101170

H -0.47045502068571

Cl 2.14144904610402

N -1.84877854015043

N -0.26480910698975

H -0.57348979953190

H 0.18980869174824

C -6.23001262019064

C -1.39479361020958

C -6.66360739581947

H -6.33522348553041

H -6.24838678113363

H $\quad-7.76169415790665$

C -6.71031028882989

H -6.29700727662080

H -6.41533921290921

H $\quad-7.80898767401609$

C -6.90714495410126

H -6.66080754175492

H -6.62706638511990

H -8.00132540231187

C -2.22305440159664

H -3.13687216659759

H -1.63571854839061

H -2.53185693175977

C -2.25839217746163

H -1.69699722997261

H -3.17358057743772

H -2.56701857439556

C -0.17766764310001

H 0.46552134750588

H 0.44038947664709

H $\quad-0.52094867046421$

C 0.73941597336894

H 1.59785659506803

H -0.09546862465836

2.82752853958138

4.18364063302298

2.46418752455369

4.89907977658406

$-3.60018115340533$

$-3.96310984283097$

$-4.49894169675816$

5.34880395861704

$-5.87864907441252$

$-6.29263751176158$

$-5.68868383497913$

$-6.61498280386157$

$-0.10655673314841$

$-0.10784081767337$

1.86513180149241

$-4.16791432092802$

$-7.36030741043176$

0.1425009810868

6.08623364753011

0.95927165965115

0.47169573016635

.97922991551285

1.04902065042180

0.86384028597147

88119337802514

0.30734981275009

0.95198110584802

$-1.24043716002419$

$-1.86321926254997$

$-1.79621748251328$

$-1.11742508788436$

6.44119852399281

5.83204803518881

6.28813052220833

7.49866772385850

6.31281032623617

6.06582802628266

5.70047354009552

7.36887435333971

7.02946095218625

6.93733280805569

6.84688455456976

8.07464275259868

$-2.92894665323644$

$-2.32463211689856$

$-2.2232978290578$

$-3.38553351710880$
0.00123139967598 0.01598504580161 $-0.07612830171734$ 0.00544023671944 $-0.09787146776416$ $-0.09400751147371$ $-0.06443184598825$ 0.02445002612474 $-0.14100643893963$ 0.08337256082652 0.14599553769761 0.21110502885323 0.14509121347727 0.14483758193411 0.20914623128723 0.14033424231788 0.25721946060754 $-0.00013098762629$ $-1.28866376496688$ 1.00781714275392 $-1.55967104997101$ 0.71492956316276 $-0.56927575564974$ $-2.55968468532085$ 1.49209257151858 0.00182002557239 $-0.02264821720568$ 0.08252787910680 2.01624075115259 $-0.80382359361554$ $-0.08826072306674$ 0.28030272986509 $-1.33122097406269$ $-2.26261133031127$ $-1.32235255845185$ $-1.36321109591024$ 1.19586789591410 1.27889239486027 2.09940073539613 1.19425410809932 $-0.15300292744193$

0.72178512375092 $-1.06209424418163$ $-0.16903086937409$ $-0.97983533794591$ $-1.06055294135377$ $-1.89889087891993$ $-0.94582052157209$

1.54633434080302 2.46113877334038 1.53896724311000

1.61144114125930 0.34526987391236 $-0.54445843335295$ 1.23882634250511

0.39345698720368 $-2.33186529143704$ $-1.99837181306035$

2.48134585904749 $-3.30374159344191$

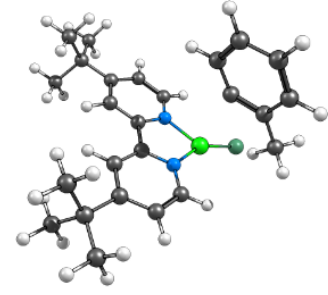

60

Aryl Chloride Triplet 3.6 A Ni-C distance

Ni $0.00001768673953 \quad-0.00001575769997$

C $2.50388778285659 \quad 1.20667502804049$

C $2.71716262083744-1.06138389069967$

C $3.89370736928023 \quad 1.34449482563069$

C $4.10814095879256-0.97952367720141$

H $2.19876293860601-1.99806161257817$

C $4.74072760272042 \quad 0.24575611790912$

$\begin{array}{lll}\text { H } 4.32200880118163 & 2.32315243169668\end{array}$

H $4.68581665187883-1.88347425513342$

C $1.51835907350625 \quad 2.28005488956824$

C $1.82080816554007 \quad 3.61935900233630$

C $0.80598723206309 \quad 4.57651725795081$

H $2.86956217822779 \quad 3.92130855280873$

C $-0.76355661937018 \quad 2.75410412743964$

C $-0.51605897897983 \quad 4.09988582537202$

H $\quad-1.78371800408436 \quad 2.36925147493148$

H $-1.37008711332649 \quad 4.76758861427485$

$\begin{array}{lll}\text { C }-0.00007937105396 & 0.00026316956645\end{array}$

C $-1.36147080642628 \quad 0.05630399012894$

C $1.00931323929683 \quad 0.02579952124489$

C $-1.70973771169216 \quad 0.15731628285259$

C $0.63168127822319 \quad 0.12490955906530$

C $-0.72819240983118 \quad 0.19110928554294$

H -2.769871722493110 .20629194499051$

$\begin{array}{lll}\text { H } & 1.39722908768625 & 0.14811063711419\end{array}$

Cl $-1.16158346072015 \quad-1.67673540532504$

N $1.91689358046771 \quad-0.00001624143615$

N $0.21784837277303 \quad 1.83565957164096$

H $2.06648503298911-0.02994487169338$

H $\quad-1.02786455215465 \quad 0.26767173025109$

C $6.26744839522669 \quad 0.41225350013574$

C $1.15685218510453 \quad 6.04454503602685$

C $6.70947322485315 \quad 1.45968303297919$

H $6.43351838053356 \quad 1.14237903995420$

H $6.25219257680102 \quad 2.44519389290782$

H $7.80341591696244 \quad 1.59147924399149$

C $6.67180113046612 \quad 0.89950377608190$

H 6.21136493679982

H $6.37061968162486 \quad 0.17421884395503$

H $7.76507021732652 \quad 1.02646133456757$

C $7.00614803566049 \quad-0.90918139703982$

H $6.75669504733337-1.69194410770971$

H $6.78298638786741-1.29639489129400$

H $8.09384013059190 \quad-0.74552281789167$

C 1.99056936890403

H $2.93059803035840 \quad 6.05645730975744$

H $1.42645784857832 \quad 6.56826683477514$

H 2.25368563866933 7.66439439454138

C $1.98640793481074 \quad 6.12520849805580$

H $1.42129174612040 \quad 5.72431553839257$

H $2.92829538255196 \quad 5.55940246752029$

H $2.24564029611414 \quad 7.17328554936989$

C $-0.10222549426171 \quad 6.91725872428035$

H $\quad-0.72404259834646 \quad 6.92717674635027$

H $-0.72760297922039 \quad 6.58163305170114$

H $0.19236330789369 \quad 7.95827288450305$

C $-2.40660955258228 \quad-0.00191594365600$

H $-3.14164105083609 \quad 0.81462152237274$

H $-1.92619739358251 \quad 0.05834147226499$

H -2.96244534585768

$-0.95343024110644$

0.00006430360850 0.28752835596190 $-0.22372704700920$ 0.33746014870825 $-0.18618426004869$ $-0.44613210675998$ 0.10138669121438 0.56700410642787 $-0.38382822581644$ 0.50371986112884 0.75766723952099 0.93883235906060 0.81234818848077

0.58448886179169 0.83957218508044 0.50862182020257 0.96158316605345 3.60006428442219 3.86485101000083 4.54418071389347

5.23259144156188 5.89754715816597 6.23062396580948 5.51026517000617 6.68051851350028 $-0.64289279826538$ 0.00675846047789 0.42380238353292 4.26410072715083 7.28028695263460 0.16218662372092 1.22541222576264 $-0.89009720934562$ $-1.90810075948306$ $-0.71082816530127$ $-0.85965841759588$ 1.57600857929103 1.82782595259829 2.34826249839823 1.63660817750000 $-0.12684743998565$ 0.60727404231546 $-1.13391532815234$ $-0.07318687304589$ 0.04915121678901 $-0.09653238269810$ $-0.89594708788360$ 0.24087526168304 2.53127767761837 3.38728104460468 2.45826665978887 2.75387255890565 1.39433499180456 0.48472371047745 2.23706089475412 1.59996722131141 2.77467386229897 2.86858748281095 1.78705557369468 


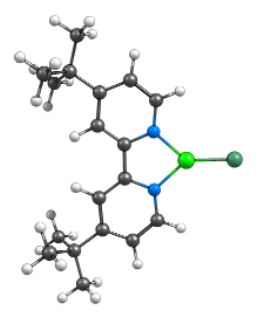

46

Aryl Chloride Putative Ni(I) Doublet-Phenyl Dissociated

$\mathrm{Ni} 1.12131625375462 \quad-1.34044278348043 \quad-0.10634848196161$

C $-0.62447086581501 \quad 0.83102322737365 \quad-0.03978139346347$

$\begin{array}{llll}\text { C } & 1.60385834795871 & 1.52979173152413 & -0.06904919896757\end{array}$

C $\begin{array}{llll}\text { C } 1.05732624177199 & 2.15867845760945 & -0.01089231366487\end{array}$

C $1.22461873705866 \quad 2.86929701629985 \quad-0.04072508602992$

H $2.65569241462020 \quad 1.23173859359291 \quad-0.09290411452293$

C $-0.13916778159110 \quad 3.22524151722763 \quad-0.01038811277372$

$\begin{array}{llll}\mathrm{H} & -2.13067376042035 & 2.36362671375235 & 0.01158757911198\end{array}$

H $2.00848615690703 \quad 3.62813389477213 \quad-0.04268621505961$

C $-1.48747078697437 \quad-0.36158915120203 \quad-0.04266927761879$

$\begin{array}{llll}\text { C } & -2.88392936937386 & -0.36563657291487 & -0.01553874376663\end{array}$

C $-3.61000752224278 \quad-1.57119228123815 \quad-0.02066986175762$

H $-3.41426373550057 \quad 0.58970747260770 \quad 0.01026451406004$

C $-1.45451993638831 \quad-2.69649333050396 \quad-0.08103189087745$

C $-2.84547947321066 \quad-2.75520675820389 \quad-0.05471662476976$

H $-0.84253252224459 \quad-3.60232159276041 \quad-0.10780721148562$

H $-3.32128593824976 \quad-3.73695749682439 \quad-0.06112053391825$

Cl $2.84902396775385 \quad-2.59273926627553 \quad-0.15646579160000$

N $0.71400908166937 \quad 0.51001102229262 \quad-0.06915199739677$

N $-0.76402843686432 \quad-1.53250318803838 \quad-0.07567497621455$

C $-0.63203620410991 \quad 4.68039948162554 \quad 0.02196402700901$

C $-5.14633863473835 \quad-1.55803858693969 \quad 0.00965773516279$

C $-1.46888147197341 \quad 4.90906824775261 \quad 1.30545260907858$

$\begin{array}{llll}H & -0.86809422441763 & 4.72512429418277 & 2.21012183934518\end{array}$

$\begin{array}{llll}H & -2.34897267495299 & 4.24863123952838 & 1.34818073690335\end{array}$

$\mathrm{H}-1.83250471909284 \quad 5.94906374990897 \quad 1.34382088802858$

C $-1.51443792393366 \quad 4.95000968280246 \quad-1.22245331838395$

$\begin{array}{llll}\mathrm{H} & -2.39588355770541 & 4.29079356649425 & -1.25484130454513\end{array}$

$\mathrm{H} \quad-0.94674255504053 \quad 4.79585198036774 \quad-2.15367068708334$

H $-1.87862980694690 \quad 5.99048018361612 \quad-1.21396580993006$

C $0.53531621483313 \quad 5.68677339064346 \quad 0.01722584507316$

H $1.15609893922674 \quad 5.59584772619511 \quad-0.88851929478285$

H $1.18817303454198 \quad 5.56701319117071 \quad 0.89668718239548$

H $\quad 0.14000136563794 \quad 6.71440680030916 \quad 0.04093570156401$

C $\begin{array}{llll}-5.62698575779308 & -0.83830419410573 & 1.29450031730898\end{array}$

H $\quad-5.27505704329918 \quad 0.20408534398353 \quad 1.34067093863975$

H $-5.26555836854063 \quad-1.35397686810619 \quad 2.19819210885678$

H $-6.72857930512399 \quad-0.81830769015551 \quad 1.33148800053130$

C $\quad-5.67640647377261 \quad-0.80068838203950 \quad-1.23347184326501$

H $\quad-5.35086013281287 \quad-1.28904709250182 \quad-2.16553147250125$

H $-5.32589842582464 \quad 0.24280972170727 \quad-1.26236114556226$

H $-6.77859598588820 \quad-0.78023171481700 \quad-1.22681362251068$

C $\quad-5.73745560564246 \quad-2.98142907749125 \quad 0.00008283309589$

$\begin{array}{llll}\text { H } & -5.42072248600976 & -3.56675927917144 & 0.87820006334712\end{array}$

H $-5.45618808281846 \quad-3.54012746023954 \quad-0.90697506315787$

H $\quad-6.83718670287697 \quad-2.92707648033080 \quad 0.02271246805954$

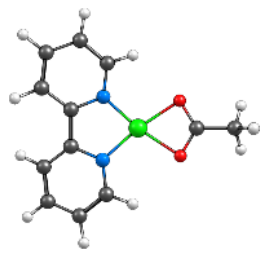

28

Aryl Acetate Putative Ni(I) Doublet-Phenyl Dissociated

C $\quad 4.645468000 \quad 0.110289000 \quad-0.075652000$

$3.849233000 \quad 1.252747000-0.027611000$

$4.025680000-1.145098000-0.249013000$

$2.642315000-1.205602000-0.367861000$

$1.857829000 \quad-0.097579000 \quad-0.363931000$

$2.457940000 \quad 1.139153000 \quad-0.179298000$

$1.490717000 \quad 2.223492000-0.159147000$

$\begin{array}{lll}0.193938000 & 1.767603000 & 0.026352000\end{array}$

$\begin{array}{lll}1.761004000 & 3.592939000 & -0.309458000\end{array}$

$4.296736000-2.233824000-0.152477000$

$\begin{array}{lll}5.729646000 & 0.185916000 & 0.038662000\end{array}$

$\begin{array}{lll}4.613314000 & -2.064528000 & -0.300636000\end{array}$

$2.105038000 \quad-2.149103000-0.493656000$

$0.715969000 \quad 4.513455000-0.262353000$

$\begin{array}{lll}2.786755000 & 3.926763000 & -0.487354000\end{array}$

$\begin{array}{lll}-0.601651000 & 4.039956000 & -0.090424000\end{array}$

$0.914550000-5.581968000-0.375804000$

$\begin{array}{lll}-0.818274000 & 2.672279000 & 0.029301000\end{array}$

$\begin{array}{lll}-1.448486000 & 4.728183000 & -0.039836000\end{array}$

$\begin{array}{lll}-1.816692000 & 2.245499000 & 0.155461000\end{array}$

$\begin{array}{lll}-0.008850000 & -0.087806000 & -0.165343000\end{array}$

$\begin{array}{lll}-0.521630000 & -2.014106000 & -0.254652000\end{array}$

$\begin{array}{lll}-1.743467000 & -1.640678000 & -0.152586000\end{array}$

$\begin{array}{lll}-1.984898000 & -0.385218000 & -0.064177000\end{array}$

$\begin{array}{lll}-2.861432000 & -2.648778000 & -0.105392000\end{array}$

$\begin{array}{lll}-2.558611000 & -3.585903000 & -0.590646000\end{array}$

$-3.764363000 \quad-2.244573000 \quad-0.583078000$

$\begin{array}{lll}-3.109648000 & -2.870474000 & 0.945508000\end{array}$ 
